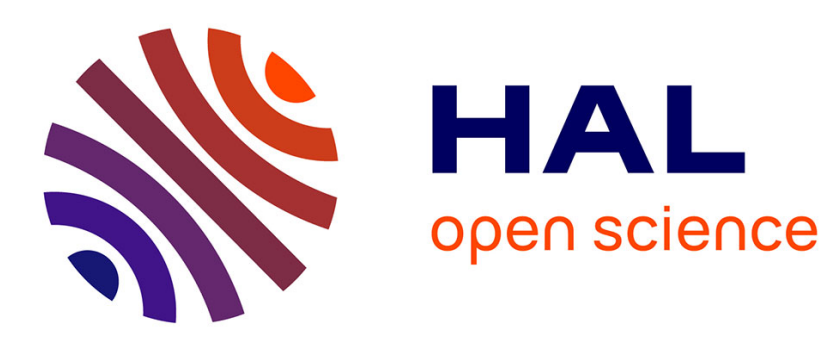

\title{
A global view of Brownian penalisations
}

Joseph Najnudel, Bernard Roynette, Marc Yor

\section{To cite this version:}

Joseph Najnudel, Bernard Roynette, Marc Yor. A global view of Brownian penalisations. Mathematical Society of Japan, pp.137, 2009, MSJ Memoirs. hal-00257600

\section{HAL Id: hal-00257600 \\ https://hal.science/hal-00257600}

Submitted on 19 Feb 2008

HAL is a multi-disciplinary open access archive for the deposit and dissemination of scientific research documents, whether they are published or not. The documents may come from teaching and research institutions in France or abroad, or from public or private research centers.
L'archive ouverte pluridisciplinaire HAL, est destinée au dépôt et à la diffusion de documents scientifiques de niveau recherche, publiés ou non, émanant des établissements d'enseignement et de recherche français ou étrangers, des laboratoires publics ou privés. 


\title{
A global view of Brownian penalisations
}

\author{
J. Najnudel ${ }^{(1)}$, B. Roynette ${ }^{(2)}$, M. Yor ${ }^{(3)(4)}$
}

$28 / 01 / 2008$

\author{
(1) ETH Zürich, Maths Dept, Zürich, CH 8092 \\ and University of Zürich CH 8092 \\ ${ }^{(2)}$ Institut Elie Cartan, Université Henri Poincaré, \\ B.P. 239, 54506 Vandoeuvre les Nancy Cedex \\ (3) Laboratoire de Probabilités et Modèles aléatoires, \\ Universités de Paris VI et VII, 4 Place Jussieu, Case 188 \\ F - 75252 Paris Cedex 05 \\ (4) Institut Universitaire de France
}

\section{Preface}

1) Let $\left(\Omega=\mathcal{C}\left(\mathbb{R}_{+} \rightarrow \mathbb{R}\right),\left(X_{t}, \mathcal{F}_{t}\right)_{t \geq 0}, \mathcal{F}_{\infty}=\underset{t \geq 0}{\vee} \mathcal{F}_{t}, W_{x}(x \in \mathbb{R})\right)$ denote the canonical realisation of one-dimensional Brownian motion. With the help of Feynman-Kac type penalisation results for Wiener measure, we have, in $[\mathrm{RY}, \mathrm{M}]$ constructed on $\left(\Omega, \mathcal{F}_{\infty}\right)$ a positive and $\sigma$-finite measure $\mathbf{W}$. The aim of this second monograph is to deepen our understanding of $\mathbf{W}$, as we discuss here other remarkable properties of this measure.

For pedagogical reasons, we have chosen to take up here again the construction of $\mathbf{W}$ found in $[R Y, M]$, so that the present monograph may be read, essentially, independently from of our previous papers, including [RY, M].

Among the main properties of $\mathbf{W}$ presented here, let us cite :

- the close links between $\mathbf{W}$ and probabilities obtained by penalising Wiener measure by certain functionals : see Theorems 1.1.2, 1.1.11 and 1.1.11';

- the existence of integral representation formulae for the measure $\mathbf{W}$ : see Theorems 1.1.6 and 1.1.8. These formulae allow to express $\mathbf{W}$ in terms of the laws of Brownian bridges and of the law of the 3-dimensional Bessel process (see, formula (1.1.43)). They also allow to express $\mathbf{W}$ in terms of the law of Brownian motion stopped at the first time when its local time at 0 reaches level $l, l$ varying, and of the law of the 3 -dimensional Bessel process (see, formula (1.1.40)). One may observe that these representation formulae are close to those obtained by Biane and Yor in $[\mathrm{B} . \mathrm{Y}]$ for some different $\sigma$-finite measures on Wiener path space.

- the existence, for every $F \in L_{+}^{1}\left(\mathcal{F}_{\infty}, \mathbf{W}\right)$, of a $\left(\left(\mathcal{F}_{t}, t \geq 0\right), W\right)$ martingale $\left(M_{t}(F), t \geq 0\right)$ which converges to 0 , as $t \rightarrow \infty$ (see Theorem 1.2.1). Many examples of such martingales are given (see Chap. 1, Examples 1 to 7). The Brownian martingales of the form $\left(M_{t}(F), t \geq 0\right)$ are characterized among the set of all Brownian martingales (see, Corollary 1.2.6) and a decomposition theorem of every positive Brownian supermartingale involving the martingales $\left(M_{t}(F), t \geq 0\right)$ is established in Theorem 1.2.5. In the same spirit, we show (see Theorem 1.2.11) that every martingale $\left(M_{t}(F), t \geq 0\right)$ with $F \in L^{1}\left(\mathcal{F}_{\infty}, \mathbf{W}\right), F$ not necessarily $\geq 0$, may be decomposed in a canonical manner into the sum of two quasi-martingales which enjoy 
some remarkable properties. In particular, this result allows to obtain a characterization of the martingales $\left(M_{t}(F), t \geq 0\right)$, with $F \in L^{1}\left(\mathcal{F}_{\infty}, \mathbf{W}\right)$ which vanish on the zero set of the process $\left(X_{t}, t \geq 0\right)$. This is Theorem 1.2.12.

- a general penalisation Theorem, for Wiener measure, which is valid for a large class of penalisation functionals $\left(F_{t}, t \geq 0\right)$ and whose proof hinges essentially upon some remarkable properties of $\mathbf{W}$ : this is the content of Theorem 1.2.14.

- the existence of invariant measures, which are intimately related with $\mathbf{W}$, for several Markov processes taking values in function spaces (see Section 1.3). Chapter 1 of this monograph is devoted to the results we have just described.

2) The results relative to the 1-dimensional Brownian motion are extended in Chapter 2, of this monograph to 2-dimensional Brownian motion (we identify $\mathbb{R}^{2}$ to $\mathbb{C}$, and use complex notation). In this framework, the role of the measure $\mathbf{W}$ is played by a positive and $\sigma$-finite measure, which we denote $\mathbf{W}^{(2)}$ on $\left(\Omega=\mathcal{C}\left(\mathbb{R}_{+}, \mathbb{C}\right), \mathcal{F}_{\infty}\right)$. The properties of $\mathbf{W}^{(2)}$ are, mutatis mutandis, analogous to those of $\mathbf{W}$. However, in the set-up of the $\mathbb{C}$-valued Brownian motion $\left(X_{t}, t \geq 0\right)$ it is of interest to consider the winding process $\left(\theta_{t}, t \geq 0\right)$ :

$$
\left(\theta_{t}, t \geq 0\right)=\left(\theta_{0}+\operatorname{Im} \int_{0}^{t} \frac{d X_{s}}{X_{s}}, t \geq 0\right)
$$

We study this process under $\mathbf{W}^{(2)}$. We then obtain a Spitzer type limit theorem about the asymptotic behavior in distribution for $\theta_{t}$, adequately normalized, as $t \rightarrow \infty$. This is Theorem 2.3.1. (see also Remark 2.3.2).

3) Chapter 3 of this Monograph is devoted to the transcription of several of the preceding results to a more general framework, that of a certain class of linear diffusions (taking values in $\mathbb{R}_{+}$). This class is described in Section 3.1. It is in fact the class of the linear diffusions studied by Salminen, Vallois and Yor (see $[\mathrm{SVY}]$ ). These are diffusions taking values in $\mathbb{R}_{+}$, and associated with a speed measure $m$ and a scale function $S$, both of which have adequate properties. Fundamental examples of such diffusions are the Bessel processes with dimension $d=2(1-\alpha)$ with $0<d<2$ ). (We also refer to $\alpha \in] 0,1[$, or to the index $-\alpha \in]-1,0[$ ). The case $d=1\left(\right.$ or $\left.\alpha=\frac{1}{2}\right)$ is that of reflected Brownian motion.

We particularize, in Section 3.3, for these examples, the general results obtained for this class of linear diffusions (see, Theorem 3.3.1). The analogue, for the Bessel process with index $(-\alpha)$, of the measure $\mathbf{W}$, is denoted $\mathbf{W}^{-\alpha}$. Then, still in this framework of the Bessel process of index $(-\alpha)$, we establish some link between, on one hand, the measure $\mathbf{W}^{-\alpha}$ and, on the other hand, a Feynman-Kac type penalisation of a Bessel process with index $(-\alpha)$ (see Remark 3.3.2 and 3.3.3). Finally, in Section 3.4, we give a new description of the measure $\mathbf{W}^{(-\alpha)}$ restricted to $\mathcal{F}_{g}$, with $g:=\sup \left\{t \geq 0 ; X_{t}=0\right\}$. This is Theorem 3.4.1. This description is the transcription in our situation of results of Pitman-Yor (see [PY]). In some sense, this description of $\mathbf{W}^{(-\alpha)}$ restricted to $\mathcal{F}_{g}$ resembles the description due to D. Williams of the Itô measure of Brownian excursions.

4) Chapter 4 of this monograph consists in obtaining, this time in the framework of Markov chains taking values in a countable set, the analogue of the preceding results. Section 4.1 is devoted to the definition of the measures $\left(\mathbb{Q}_{x}, x \in E\right)$ which play here the role of the measures $\mathbf{W}_{x}$ in the precedings chapters. Also as in the preceding chapters, certain martingales are associated to these measures $\left(\mathbb{Q}_{x}, x \in E\right)$, as described in Corollary 4.2.2. In this 
new framework of the Markov chains, the $\sigma$-finite measures $\left(\mathbb{Q}_{x}, x \in E\right)$ depend, from our construction, on a point $x_{0} \in E$ and on a funtion $\Phi$. This dependence with respect to $x_{0}$ and $\Phi$ is studied in subsections 4.2.3 and 4.2.4. Section 4.3 is devoted to the study of many examples ; in particular, for random walks on trees, it appears that there may exist a whole family of different measures $\left(\mathbb{Q}_{x}, x \in E\right)$. All the results found in this Chapter 4 are due solely to J. Najnudel. 


\section{Table of contents}

Chap. 1 Existence and main properties of $\mathbf{W}$

1.0 Introduction

1.1 Existence of $\mathbf{W}$ and first properties

1.1.1 A few more notations

1.1.2 A Feynman-Kac penalisation result

1.1.3 Definition of $\mathbf{W}$

1.1.4 Study of the canonical process under $W_{\infty}^{\left(\lambda \delta_{0}\right)}$

1.1.5 Some remarkable properties of $\mathbf{W}$

1.1.6 Another approach to Theorem 1.6

1.1.7 Relation between $\mathbf{W}$ and other penalisations (than the Feynman-Kac ones)

1.2 Brownian martingales associated to $\mathbf{W}$

1.2.1 Definition of the martingales $\left(M_{t}(F), t \geq 0\right)$

1.2.2 Examples of martingales $\left(M_{t}(F), t \geq 0\right)$

1.2.3 A decomposition Theorem of positive Brownian supermartingales

1.2.4 A decompositon result of the martingale $\left(M_{t}(F), t \geq 0\right)$

1.2.5 A penalisation Theorem

1.3 Invariant measures related to $\mathbf{W}_{x}$ and $\boldsymbol{\Lambda}_{x}$

1.3.1 The process $\left(\mathcal{X}_{t}, t \geq 0\right)$

1.3.2 The measure $\boldsymbol{\Lambda}_{x}$

1.3.3 Invariant measures for the process $\left(\left(X_{t}, L_{t}^{\bullet}\right), t \geq 0\right)$

1.3.4 Invariant measures for the process $\left(L_{t}^{X_{t^{-}} \bullet}, t \geq 0\right)$

Chap. 2 Existence and properties of $\mathbf{W}^{(2)}$

2.1 Existence of $\mathbf{W}^{(\mathbf{2})}$

2.1.1 Notation and Feynman-Kac penalisations in two dimensions

2.1.2 Existence of the measure $\mathbf{W}^{(\mathbf{2})}$

2.2 Properties of $\mathbf{W}^{(\mathbf{2})}$ 


\subsubsection{Some notation}

2.2.2 Description of the canonical process under $W_{\infty}^{\left(2, q_{0}\right)}$

2.2.3 Another description of the measure $\mathbf{W}^{(\mathbf{2})}$

2.3 Study of the winding process under $\mathbf{W}^{(\mathbf{2})}$

2.3.1 Spitzer's Theorem

2.3.2 An analogue of Spitzer's Theorem

$2.4 \underline{W^{(2)} \text {-martingales associated to } \mathbf{W}^{(2)}}$

2.4.1 Definition of $\left(M_{t}^{(2)}(F), t \geq 0\right)$

2.4.2 A decomposition Theorem for positive $W^{(2)}$ supermartingales

2.4.3 A decomposition Theorem of the martingale $\left(M_{t}^{(2)}(F), t \geq 0\right)$

Chap. 3 The analogue of the measure $\mathbf{W}$ for a class of linear diffusions

3.1 Main hypotheses and notations

3.1.1 Our framework

3.1.2 The semi-group of $\left(X_{t}, t \geq 0\right)$

3.1.3 The local time process

3.1.4 The process $X$ conditionned not to vanish

3.1.5 A useful Proposition

3.2 The $\sigma$-finite measure $\mathbf{W}^{*}$

3.2.1 Definition of $\mathbf{W}^{*}$

3.2.2 Some properties of $\mathbf{W}^{*}$

3.2.2.1 The probabilities $P_{x, \infty}^{(\lambda)}$

3.2.2.2 The measures $\left(\mathbf{W}_{x}^{*}, x \in E\right)$

3.2.2.3 Martingales associated with $\left(\mathbf{W}_{x}^{*}, x \in \mathbb{R}_{+}\right)$

3.2.2.4 A decomposition Theorem for $\left(\left(\mathcal{F}_{t}, t \geq 0\right), P_{x}\right)$ supermartingales

3.2.3 Relation between $\mathbf{W}^{*}$ and penalisations

3.3 The example of Bessel process of dimension $d(0<d<2)$

3.3.1 Transcription of our notation in the context of Bessel process 
3.3.2 The measure $\mathbf{W}^{(-\alpha)}$

3.3.3 Relations between $\mathbf{W}^{(-\alpha)}(\alpha=2(1-\alpha))$ and Feynman-Kac penalisations

3.4 Another description of $\mathbf{W}^{*}$ and $\mathbf{W}^{(-\alpha)}$

3.5 Penalisations of $\alpha$-stable symmetric process $(1<\alpha \leq 2)$

3.5.1 Notation and classical results

3.5.2 Definition of the $\sigma$-finite measure $\mathbf{P}$

3.5.3 The martingales $\left(M_{t}(F), t \geq 0\right)$ assciated with $\mathbf{P}$

3.5.4 Relations between $\mathbf{P}$ and penalisations

Chap. 4 An analogue of the measure $\mathbf{W}$ for discrete Markov chains

4.0 Introduction

4.1 Construction of the $\sigma$-finite measures $\left(\mathbb{Q}_{x}, x \in E\right)$

4.1.1 Notation and hypothesis

4.1.2 A family of new probabilities

4.1.3 Definition of the measures $\left(\mathbb{Q}_{x}, x \in E\right)$

4.2 Some properties of $\left(\mathbb{Q}_{x}, x \in E\right)$

4.2.1 Martingales associated with $\left(\mathbb{Q}_{x}, x \in E\right)$

4.2.2 Properties of the canonical process under $\left(\mathbb{Q}_{x}, x \in E\right)$

4.2.3 Dependence of $\mathbb{Q}_{x}$ on $x$

4.2.4 Dependence of $\mathbb{Q}_{x}$ on $\Phi$

4.3 Some examples

4.3.1 $\Phi$ is a bounded function

4.3.2 Example of the standard random walk

4.3.3 Example of the "bang-bang random walk"

4.3.4 Example of random walk on a tree

4.3.5 Some sufficient conditions for the existence of $\Phi$

4.3.6 Example of the standard random walk on $\mathbb{Z}^{2}$ 


\section{Chapter 1. On a remarkable $\sigma$-finite measure $\mathrm{W}$ on path space, which rules penalisations for linear Brownian motion}

\subsection{Introduction.}

1.0.1 $\left(\Omega,\left(X_{t}, \mathcal{F}_{t}\right), t \geq 0, \mathcal{F}_{\infty}, W_{x}(x \in \mathbb{R})\right)$ denotes the canonical realisation of 1-dimensional Brownian motion. $\Omega=\mathcal{C}\left(\mathbb{R}_{+} \rightarrow \mathbb{R}\right),\left(X_{t}, t \geq 0\right)$ is the coordinate process on this space and $\left(\mathcal{F}_{t}, t \geq 0\right)$ denotes its natural filtration $; \mathcal{F}_{\infty}=\underset{t \geq 0}{\vee} \mathcal{F}_{t}$. For every $x \in \mathbb{R}, W_{x}$ denotes Wiener measure on $\left(\Omega, \mathcal{F}_{\infty}\right)$ such that $W_{x}\left(X_{0}=x\right)=1$. We write $W$ for $W_{0}$ and if $Z$ is a r.v. defined on $\left(\Omega, \mathcal{F}_{\infty}\right)$, we write $W_{x}(Z)$ for the expectation of $Z$ under the probability $W_{x}$.

1.0.2 In a series of papers ([RVY, $i], i=I, I I, \cdots, V I I I)$ we have studied various penalisations of Wiener measure with certain positive functionals $\left(F_{t}, t \geq 0\right)$; that is for each functional $\left(F_{t}, t \geq 0\right)$ in a certain class, we have been able to show the existence of a probability $W_{\infty}^{F}$ on $\left(\Omega, \mathcal{F}_{\infty}\right)$ such that : for every $s \geq 0$ and every $\Gamma_{s} \in b\left(\mathcal{F}_{s}\right)$, the space of bounded $\mathcal{F}_{s}$ measurable variables :

$$
\lim _{t \rightarrow \infty} \frac{W\left(\Gamma_{s} F_{t}\right)}{W\left(F_{t}\right)}=W_{\infty}^{F}\left(\Gamma_{s}\right)
$$

In this paper, we shall construct a positive and $\sigma$-finite measure $\mathbf{W}$ on $\left(\Omega, \mathcal{F}_{\infty}\right)$ which, in some sense, "rules all these penalisations jointly".

1.0.3 In Section 1.1 of this chapter, we show the existence of $\mathbf{W}$ and we describe some of its properties.

In Section 1.2, we show how to associate to $\mathbf{W}$ a family of $\left(\left(\mathcal{F}_{t}, t \geq 0\right), W\right)$ martingales $\left(M_{t}(F), t \geq 0\right)\left(F \in L_{+}^{1}\left(\mathcal{F}_{\infty}, \mathbf{W}\right)\right)$. We study the properties of these martingales and give many examples.

In Section 1.3, we describe links between $\mathbf{W}$ and a $\sigma$-finite measure $\boldsymbol{\Lambda}$ which is defined as the "law" of the total local time of the canonical process under $\mathbf{W}$ in Chapter 3 of $[R Y, M]$. In particular, we construct an invariant measure $\widetilde{\boldsymbol{\Lambda}}$ for the Markov process $\left(\left(X_{t}, L_{t}^{\bullet}\right), t \geq 0\right)$ (and $\widetilde{\boldsymbol{\Lambda}}$ is intimately related to $\boldsymbol{\Lambda})$. Here, $L_{t}^{\bullet}$ denotes the local times process $\left(L_{t}^{x}, x \in \mathbb{R}_{+}\right)$, so that this Markov process $\left(X, L^{\bullet}\right)$ takes values in $\mathbb{R} \times \mathcal{C}\left(\mathbb{R} \longrightarrow \mathbb{R}_{+}\right)$.

1.0.4 Notations : As certain $\sigma$-finite measures play a prominent role in our paper, we denote them, as a rule, in bold characters. Thus, no confusion should arise between the $\sigma$-finite measure $\mathbf{W}_{x}$ and the Wiener measure $W_{x}$.

\subsection{Existence of $\mathrm{W}$ and first properties.}

Our aim in this section is to define, via Feynman-Kac type penalisations, a positive and $\sigma$ finite measure $\mathbf{W}$ on $\left(\Omega, \mathcal{F}_{\infty}\right)$. Moreover, independently from this penalisation procedure, we give several remarkable descriptions of $\mathbf{W}$.

\subsubsection{A few more notations.}

$\left(\Omega,\left(X_{t}, \mathcal{F}_{t}\right)_{t \geq 0}, \mathcal{F}_{\infty}, W_{x}(x \in \mathbb{R})\right)$ denotes the canonical realisation of 1-dimensional Brownian motion.

We denote by $\mathcal{I}$ the set of positive Radon measures $q(d x)$ on $\mathbb{R}$, such that :

$$
0<\int_{0}^{\infty}(1+|x|) q(d x)<\infty
$$


For every $q \in \mathcal{I},\left(A_{t}^{(q)}, t \geq 0\right)$ denotes the additive functional defined by :

$$
A_{t}^{(q)}:=\int_{\mathbb{R}} L_{t}^{y} q(d y)
$$

where $\left(L_{t}^{y}, t \geq 0, y \in \mathbb{R}\right)$ denotes the jointly continuous family of local times of Brownian motion $\left(X_{t}, t \geq 0\right)$. When the Radon measure $q$ admits a density with respect to the Lebesgue measure on $\mathbb{R}$ - and then we denote again this density by $q$ - the density of occupation formula yields :

$$
A_{t}^{(q)}=\int_{\mathbb{R}} L_{t}^{y} q(d y)=\int_{0}^{t} q\left(X_{s}\right) d s
$$

We denote by $b\left(\mathcal{F}_{s}\right)\left(\right.$ resp. $\left.b_{+}\left(\mathcal{F}_{s}\right)\right)$ the vector space of bounded and real valued (resp. the cone of bounded and positive) $\mathcal{F}_{s}$ measurable r.v.'s.

As our means to construct $\mathbf{W}$, we use a penalisation result obtained in [RVY, I] (see also $[R Y, M])$. In the next subsection, we recall this result.

\subsubsection{A Feynman-Kac penalisation result.}

Theorem 1.1.1. Let $q \in \mathcal{I}$ and:

$$
\begin{aligned}
D_{x, t}^{(q)} & :=W_{x}\left(\exp \left(-\frac{1}{2} A_{t}^{(q)}\right)\right) \\
W_{x, t}^{(q)} & :=\frac{\exp \left(-\frac{1}{2} A_{t}^{(q)}\right)}{D_{x, t}^{(q)}} \cdot W_{x}
\end{aligned}
$$

1) For every $s \geq 0$ and $\Gamma_{s} \in b\left(\mathcal{F}_{s}\right), W_{x, t}^{(q)}\left(\Gamma_{s}\right)$ admits a limit as $t \longrightarrow \infty$, denoted by $W_{x, \infty}^{(q)}\left(\Gamma_{s}\right)$, i.e. :

$$
W_{x, t}^{(q)}\left(\Gamma_{s}\right) \underset{t \rightarrow \infty}{\longrightarrow} W_{x, \infty}^{(q)}\left(\Gamma_{s}\right)
$$

We express this property by writing that $W_{x, t}^{(q)}$ converges, as $t \longrightarrow \infty$, to $W_{x, \infty}^{(q)}$ along the filtration $\left(\mathcal{F}_{s}, s \geq 0\right)$.

2) $W_{x, \infty}^{(q)}$ induces a probability on $\left(\Omega, \mathcal{F}_{\infty}\right)$ such that :

$$
W_{x,\left.\infty\right|_{\mathcal{F}_{s}}}^{(q)}=M_{x, s}^{(q)} \cdot W_{\left.x\right|_{\mathcal{F}_{s}}}
$$

where $\left(M_{x, s}^{(q)}, s \geq 0\right)$ is the $\left(\left(\mathcal{F}_{s}, s \geq 0\right), W_{x}\right)$ martingale defined by :

$$
M_{x, s}^{(q)}:=\frac{\varphi_{q}\left(X_{s}\right)}{\varphi_{q}(x)} \exp \left(-\frac{1}{2} A_{s}^{(q)}\right)
$$

In particular, $M_{x, 0}^{(q)}=1 \quad W_{x}$ a.s.

The function $\varphi_{q}: \mathbb{R} \longrightarrow \mathbb{R}_{+}$which is featured in (1.1.8) is strictly positive, continuous, convex and satisfies :

$$
\varphi_{q}(x) \underset{|x| \rightarrow \infty}{\sim}|x|
$$

3) $\varphi_{q}$ may be defined via one or the other of the two following properties : 
i) $\varphi_{q}$ is the unique solution of the Sturm-Liouville equation:

$$
\varphi^{\prime \prime}=\varphi \cdot q \quad \text { (in the sense of distributions) }
$$

which satisfies the boundary conditions :

$$
\varphi^{\prime}(+\infty)=-\varphi^{\prime}(-\infty)=1
$$

ii) $\sqrt{\frac{\pi t}{2}} W_{x}\left(\exp \left(-\frac{1}{2} A_{t}^{(q)}\right)\right) \underset{t \rightarrow \infty}{\longrightarrow} \varphi_{q}(x)$

4) Under the family of probabilities $\left(W_{x, \infty}^{(q)}, x \in \mathbb{R}\right)$, the canonical process $\left(X_{t}, t \geq 0\right)$ is a transient time homogeneous diffusion. More precisely, there exists a $\left(\Omega,\left(\mathcal{F}_{t}, t \geq 0\right), W_{\infty}^{(q)}\right)$ Brownian motion $\left(B_{t}, t \geq 0\right)$ such that :

$$
X_{t}=x+B_{t}+\int_{0}^{t} \frac{\varphi_{q}^{\prime}\left(X_{s}\right)}{\varphi_{q}\left(X_{s}\right)} d s
$$

In particular, this diffusion process $\left(X_{t}, t \geq 0\right)$ admits the following function $\gamma_{q}$ as its scale function :

$$
\gamma_{q}(x):=\int_{0}^{x} \frac{d y}{\varphi_{q}^{2}(y)}
$$

(and : $\left.\left|\gamma_{q}( \pm \infty)\right|<\infty\right)$.

We note that the function $\varphi_{q}$ featured in Theorem 1.1 is not exactly the one found in [RY, $\mathrm{M}]$. It differs from it by the factor $\sqrt{\frac{\pi}{2}}$; we have made this slight change in order to simplify some further formulae.

\subsubsection{Definition of $\mathrm{W}$.}

We now use Theorem 1.1.1 to construct the $\sigma$-finite measure $\mathbf{W}$. In fact, we define, for every $x \in \mathbb{R}$, a positive and $\sigma$-finite $\mathbf{W}_{x}$ which is deduced from $\mathbf{W}$ via the following simple translation by $x$ :

$$
\mathbf{W}_{x}\left(F\left(X_{s}, s \geq 0\right)\right)=\mathbf{W}\left(F\left(x+X_{s}, s \geq 0\right)\right)
$$

for every positive functional $F$. This formula (1.1.15) explains why, most of the time, we may limit ourselves to consider $\mathbf{W}_{0}$, which we denote simply by $\mathbf{W}$.

Theorem 1.1.2. (Existence of $\mathbf{W}$ )

There exists, on $\left(\Omega, \mathcal{F}_{\infty}\right)$ a positive and $\sigma$-finite measure $\mathbf{W}$, with infinite total mass, such that, for every $q \in \mathcal{I}$ :

$$
\mathbf{W}=\varphi_{q}(0) \exp \left(\frac{1}{2} A_{\infty}^{(q)}\right) \cdot W_{\infty}^{(q)}
$$

In other terms, the RHS of (1.1.16) does not depend on $q \in \mathcal{I}$. In particular :

$$
\mathbf{W}\left(\exp \left(-\frac{1}{2} A_{\infty}^{(q)}\right)\right)=\varphi_{q}(0)
$$


As we shall soon see, the measure $\mathbf{W}$ is such that, for every $t>0$ and for every r.v. $\Gamma_{t} \in$ $b_{+}\left(\mathcal{F}_{t}\right), \mathbf{W}\left(\Gamma_{t}\right)$ equals 0 or $+\infty$ depending whether $W\left(\Gamma_{t}\right)=0$ or is strictly positive. Thus, the measure $\mathbf{W}$, although, as we show later, it is $\sigma$-finite on $\left(\Omega, \mathcal{F}_{\infty}\right)$, is not $\sigma$-finite on either of the measurable spaces $\left(\Omega, \mathcal{F}_{t}\right), t>0$.

Proof of Theorem 1.1.2.

i) We shall establish that, for every $q \in \mathcal{I}$, the measure on $\left(\Omega, \mathcal{F}_{\infty}\right)$ :

$$
\varphi_{q}(0) \exp \left(\frac{1}{2} A_{\infty}^{(q)}\right) \cdot W_{\infty}^{(q)}
$$

does not depend on $q$, which allows to define $\mathbf{W}$ from formula (1.1.16). Then, we shall prove that $\mathbf{W}$, thus defined, is $\left(\Omega, \mathcal{F}_{\infty}\right) \sigma$-finite.

ii) Lemma 1.1.3. For every $q \in \mathcal{I}$ and every $x \in \mathbb{R}$ :

$$
\begin{aligned}
& \text { 1) } \text { if } \lambda<1 \quad W_{x, \infty}^{(q)}\left(\exp \frac{\lambda}{2} A_{\infty}^{(q)}\right)<\infty \\
& 2) \text { if } \lambda \geq 1 \quad W_{x, \infty}^{(q)}\left(\exp \frac{\lambda}{2} A_{\infty}^{(q)}\right)=+\infty
\end{aligned}
$$

\section{Proof of Lemma 1.1.3.}

From (1.1.7), for every $\lambda \in] 0,1[$ :

$$
\begin{aligned}
& W_{x, \infty}^{(q)}\left(\exp \frac{\lambda}{2} A_{t}^{(q)}\right)=W_{x}\left(\frac{\varphi_{q}\left(X_{t}\right)}{\varphi_{q}(x)} \exp \left(-\left(\frac{1-\lambda}{2}\right) A_{t}^{(q)}\right)\right) \\
& =\frac{\varphi_{(1-\lambda) q}(x)}{\varphi_{q}(x)} W_{x}\left(\frac{\varphi_{q}\left(X_{t}\right)}{\varphi_{(1-\lambda) q}\left(X_{t}\right)} \frac{\varphi_{(1-\lambda) q}\left(X_{t}\right)}{\varphi_{(1-\lambda) q}(x)} \exp \left(-\left(\frac{1-\lambda}{2}\right) A_{t}^{(q)}\right)\right)
\end{aligned}
$$

We have been able to write (1.1.20) because the functions $\varphi_{q}$ and $\varphi_{(1-\lambda) q}$ are strictly positive. On the other hand, since for every $q \in \mathcal{I}, \varphi_{q}(x) \underset{|x| \rightarrow \infty}{\sim}|x|$, there exist two constants :

$$
0<C_{1}(\lambda, q) \leq C_{2}(\lambda, q)<\infty
$$

such that :

$$
C_{1}(\lambda, q) \leq \inf _{y \in \mathbb{R}} \frac{\varphi_{q}(y)}{\varphi_{(1-\lambda) q}(y)} \leq \sup _{y \in \mathbb{R}} \frac{\varphi_{q}(y)}{\varphi_{(1-\lambda) q(y)}} \leq C_{2}(\lambda, q)
$$

Thus, from (1.1.20) :

$$
W_{x, \infty}^{(q)}\left(\exp \frac{\lambda}{2} A_{t}^{(q)}\right) \leq \frac{\varphi_{(1-\lambda) q}(x)}{\varphi_{q}(x)} \sup _{y \in \mathbb{R}} \frac{\varphi_{q}(y)}{\varphi_{(1-\lambda) q}(y)} W_{x, \infty}^{(q)}(1) \leq \frac{C_{2}(\lambda, q)}{C_{1}(\lambda, q)}
$$

We now let $t \longrightarrow \infty$ and we use the monotone convergence Theorem to obtain point 1 ) of Lemma 1.1.3.

We now write relation (1.1.20) with $\lambda=1$ :

$$
W_{x, \infty}^{(q)}\left(\exp \frac{1}{2} A_{t}^{(q)}\right)=W_{x}\left(\frac{\varphi_{q}\left(X_{t}\right)}{\varphi_{q}(x)}\right) \underset{t \rightarrow \infty}{\sim} k(x) \sqrt{t}
$$


with $k(x)=\frac{1}{\varphi_{q}(x)} \cdot \sqrt{\frac{2}{\pi}}>0$, since $\varphi_{q}(x) \underset{|x| \rightarrow \infty}{\sim}|x|$. It then remains to let $t \longrightarrow \infty$ in (1.1.23), then to apply once again the monotone convergence Theorem to obtain point 2) of Lemma 1.1.3.

iii) Formula (1.1.16) is then a consequence of :

Lemma 1.1.4. The measure $\varphi_{q}(x) \exp \left(\frac{1}{2} A_{\infty}^{(q)}\right) \cdot W_{x, \infty}^{(q)}$ does not depend on $q \in \mathcal{I}$.

We note that the measure $\varphi_{q}(x) \exp \left(\frac{1}{2} A_{\infty}^{(q)}\right) \cdot W_{x, \infty}^{(q)}$ is well defined since, from point 1) of Lemma 1.1.3, the r.v. $A_{\infty}^{(q)}$ is $W_{x, \infty}^{(q)}$ a.s. finite. On the other hand, the measure $\varphi_{q}(x) \exp \left(\frac{1}{2} A_{\infty}^{(q)}\right) \cdot W_{x, \infty}^{(q)}$ has infinite total mass from point 2) of Lemma 1.1.3.

\section{Proof of Lemma 1.1.4.}

Let $q_{1}, q_{2} \in \mathcal{I}$. Then, from (1.1.17) and (1.1.18) we have for every $\Gamma_{u} \in b_{+}\left(\mathcal{F}_{u}\right)$, with $u \leq t$ :

$$
\begin{aligned}
W_{x, \infty}^{\left(q_{1}\right)}\left(\Gamma_{u} \varphi_{q_{1}}(x) \exp \left(\frac{1}{2} A_{t}^{\left(q_{1}\right)}\right)\right) & =W_{x}\left(\Gamma_{u} \varphi_{q_{1}}\left(X_{t}\right)\right) \\
& =W_{x}\left(\Gamma_{u} \frac{\varphi_{q_{1}}\left(X_{t}\right)}{\varphi_{q_{2}}\left(X_{t}\right)} \varphi_{q_{2}}\left(X_{t}\right)\right) \\
& =W_{x, \infty}^{\left(q_{2}\right)}\left(\Gamma_{u} \varphi_{q_{2}}(x) \frac{\varphi_{q_{1}}\left(X_{t}\right)}{\varphi_{q_{2}}\left(X_{t}\right)} \exp \left(\frac{1}{2} A_{t}^{\left(q_{2}\right)}\right)\right)
\end{aligned}
$$

Since the relation (1.1.24) takes place for every $\Gamma_{u} \in b_{+}\left(\mathcal{F}_{u}\right)$ for any $u \leq t$, we may replace $\Gamma_{u}$ by $\Gamma_{u} \exp \left(-\varepsilon A_{t}^{\left(q_{1}+q_{2}\right)}\right) \quad(\varepsilon>0)$. We obtain :

$$
\begin{aligned}
& W_{x, \infty}^{\left(q_{1}\right)}\left[\Gamma_{u} \varphi_{q_{1}}(x) \exp \left(\left(\frac{1}{2}-\varepsilon\right) A_{t}^{\left(q_{1}\right)}\right) \cdot \exp \left(-\varepsilon A_{t}^{\left(q_{2}\right)}\right)\right] \\
& \quad=W_{x, \infty}^{\left(q_{2}\right)}\left[\Gamma_{u} \varphi_{q_{2}}(x) \frac{\varphi_{q_{1}}\left(X_{t}\right)}{\varphi_{q_{2}}\left(X_{t}\right)} \exp \left(\left(\frac{1}{2}-\varepsilon\right) A_{t}^{\left(q_{2}\right)}\right) \cdot \exp \left(-\varepsilon A_{t}^{\left(q_{1}\right)}\right)\right]
\end{aligned}
$$

However - this is point 4) of Theorem 1.1.1 - $\left|X_{t}\right| \underset{t \rightarrow \infty}{\longrightarrow} \infty W_{x, \infty}^{\left(q_{2}\right)}$ a.s. and the function $x \longrightarrow \frac{\varphi_{q_{1}}(x)}{\varphi_{q_{2}}(x)}$ is bounded and tends to 1 when $|x| \longrightarrow \infty$. The dominated convergence Theorem - which we may apply thanks to Lemma 1.1.3 - implies then, by letting $t \longrightarrow \infty$ in $(1.1 .25)$ :

$$
\begin{aligned}
& \varphi_{q_{1}}(x) W_{x, \infty}^{\left(q_{1}\right)}\left[\Gamma_{u} \exp \left(\left(\frac{1}{2}-\varepsilon\right) A_{\infty}^{\left(q_{1}\right)}\right) \exp \left(-\varepsilon A_{\infty}^{\left(q_{2}\right)}\right)\right] \\
& =\varphi_{q_{2}}(x) W_{x, \infty}^{\left(q_{2}\right)}\left[\Gamma_{u}\left(\exp \left(\left(\frac{1}{2}-\varepsilon\right) A_{\infty}^{\left(q_{2}\right)}\right) \cdot \exp \left(-\varepsilon A_{\infty}^{\left(q_{1}\right)}\right)\right]\right.
\end{aligned}
$$

Since (1.1.26) holds for every $\Gamma_{u} \in b_{+}\left(\mathcal{F}_{u}\right)$ the monotone class Theorem implies that (1.1.26) is still true when we replace $\Gamma_{u} \in b_{+}\left(\mathcal{F}_{u}\right)$ by $\Gamma \in b_{+}\left(\mathcal{F}_{\infty}\right)$. It then remains to let $\varepsilon \longrightarrow 0$ and to use the monotone convergence Theorem to obtain : for every $\Gamma \in b_{+}\left(\mathcal{F}_{\infty}\right)$ :

$$
\varphi_{q_{1}}(x) W_{x, \infty}^{\left(q_{1}\right)}\left(\Gamma \exp \left(\frac{1}{2} A_{\infty}^{\left(q_{1}\right)}\right)\right)=\varphi_{q_{2}}(x) W_{x, \infty}^{\left(q_{2}\right)}\left(\Gamma \exp \left(\frac{1}{2} A_{\infty}^{\left(q_{2}\right)}\right)\right)
$$


This is Lemma 1.1.4 and point 1) of Theorem 1.1.2.

iv) We now show that $\mathbf{W}$ has infinite mass, but is $\sigma$-finite on $\mathcal{F}_{\infty}$.

Firstly, it is clear, from point 2) of Lemma 1.1.3, that :

$$
\mathbf{W}(1)=\varphi_{q}(0) W_{\infty}^{(q)}\left(\exp \left(\frac{1}{2} A_{\infty}^{(q)}\right)\right)=+\infty
$$

On the other hand, from point 1) of Lemma 1.1.3, $A_{\infty}^{(q)}<\infty W_{\infty}^{(q)}$ a.s. Hence :

$$
1_{A_{\infty}^{(q)} \leq n} \uparrow 1 \quad W_{\infty}^{(q)} \quad \text { a.s. }
$$

Thus :

$$
\mathbf{W}\left(A_{\infty}^{(q)} \leq n\right)=\varphi_{q}(0) W_{\infty}^{(q)}\left(\left(\exp \left(\frac{1}{2} A_{\infty}^{(q)}\right)\right) \cdot 1_{A_{\infty}^{(q)} \leq n}\right) \leq \varphi_{q}(0) e^{\frac{n}{2}}
$$

which proves that $\mathbf{W}$ is $\left(\Omega, \mathcal{F}_{\infty}\right) \sigma$-finite.

v) We now show that, for every $\Gamma_{t} \in b_{+}\left(\mathcal{F}_{t}\right), \mathbf{W}\left(\Gamma_{t}\right)=0$ or $+\infty$.

By definition of $\mathbf{W}$, we have :

$$
\begin{aligned}
\mathbf{W}\left(\Gamma_{t}\right) & =\varphi_{q}(0) W_{\infty}^{(q)}\left(\Gamma_{t} \exp \left(\frac{1}{2} A_{\infty}^{(q)}\right)\right) \\
& =\varphi_{q}(0) W_{\infty}^{(q)}\left(\Gamma_{t} \exp \left(\frac{1}{2} A_{t}^{(q)}\right) W_{X_{t}, \infty}^{(q)}\left(\exp \left(\frac{1}{2} A_{\infty}^{q}\right)\right)\right)
\end{aligned}
$$

from the Markov property. But, from Lemma 1.1.3, $W_{x, \infty}^{(q)}\left(\exp \left(\frac{1}{2} A_{\infty}^{(q)}\right)\right)=+\infty$ for every $x \in \mathbb{R}$. Thus, $\mathbf{W}\left(\Gamma_{t}\right)$ equals 0 or $+\infty$ according to whether $W_{\infty}^{(q)}\left(\Gamma_{t}\right)$ is 0 or is strictly positive, i.e. according to whether $W\left(\Gamma_{t}\right)$ equals 0 or is strictly positive since, from (1.1.7) and (1.1.8), the probabilities $W$ and $W_{\infty}^{(q)}$ are equivalent on $\mathcal{F}_{t}$.

The careful reader may have been surprised about our use in the proof of Lemma 1.1.4 of the r.v. $\exp \left(-\varepsilon A_{t}^{\left(q_{1}+q_{2}\right)}\right)$. This is purely technical and "counteracts" the fact that $\mathbf{W}$ takes only the values 0 and $+\infty$ on $\mathcal{F}_{t}$.

We shall now give several other descriptions of the measure W. In order to obtain these descriptions we use a particular case of Theorem 1.1.1, which shall play a key role in our study. This particular case is that of $q=\delta_{0}$ (or more generally $q=\lambda \delta_{0}$ ), the Dirac measure in 0 . We begin by recalling a result in this case.

\subsubsection{Study of the canonical process under $W_{\infty}^{\left(\lambda \delta_{0}\right)}$.}

Theorem 1.1.5 below has been obtained in [RVY, II], Theorem 8, p. 339, with $h^{+}(x)=$ $h^{-}(x)=\exp \left(-\frac{\lambda x}{2}\right) \quad(\lambda, x \geq 0)$.

Theorem 1.1.5. (A particular case of Theorem 1.1.1, with $q=\lambda \delta_{0}$, hence $\left(A_{t}^{(q)}=\lambda L_{t}, t \geq 0\right.$ ) where $\left(L_{t}, t \geq 0\right)$ is the Brownian local time at 0 .)

1) The function $\varphi_{\lambda \delta_{0}}$ defined by (1.1.10), (1.1.11) equals:

$$
\varphi_{\lambda \delta_{0}}(x)=|x|+\frac{2}{\lambda} ; \quad \text { hence, } \quad \varphi_{\lambda \delta_{0}}(0)=\frac{2}{\lambda}
$$


while the martingale $\left(M_{s}^{\left(\lambda \delta_{0}\right)}, s \geq 0\right)($ see (1.1.18)) equals :

$$
M_{s}^{\left(\lambda \delta_{0}\right)}=\left(1+\frac{\lambda}{2}\left|X_{s}\right|\right) \quad \exp \left(-\frac{\lambda}{2} L_{s}\right)
$$

2) Under $W_{\infty}^{\left(\lambda \delta_{0}\right)}$ :

i) The r.v. $g:=\sup \left\{u \geq 0 ; X_{u}=0\right\}$ is $W_{\infty}^{\left(\lambda \delta_{0}\right)}$ a.s. finite and $L_{\infty}\left(=L_{g}\right)$ has density :

$$
f_{L_{\infty}}^{W_{\infty}^{\left(\lambda \delta_{0}\right)}}(l)=\frac{\lambda}{2} e^{-\frac{\lambda}{2} l} 1_{[0, \infty[}(l)
$$

ii) The processes $\left(X_{u}, u \leq g\right)$ and $\left(X_{g+u}, u \geq 0\right)$ are independent.

iii) The process $\left(X_{g+u}, u \geq 0\right)$ is distributed with $P_{0}^{(3, \mathrm{sym})}$ where:

$$
P_{0}^{(3, \text { sym })}=\frac{1}{2}\left(P_{0}^{(3)}+\widetilde{P}_{0}^{(3)}\right)
$$

with $P_{0}^{(3)}$ (resp. $\left.\widetilde{P}_{0}^{(3)}\right)$ denoting the law of 3-dimensional Bessel process (resp. its opposite) starting from 0.

iv) Conditionally on $L_{\infty}\left(=L_{g}\right)=l,\left(X_{u}, u \leq g\right)$ is a Brownian motion starting from 0 , considered until its local time at 0 reaches level $l$, that is up to the stopping time:

$$
\tau_{l}:=\inf \left\{t \geq 0 ; L_{t}>l\right\}
$$

We write $W_{0}^{\tau_{l}}$ for the law of this process.

$$
W_{\infty}^{\left(\lambda \delta_{0}\right)}=\frac{\lambda}{2} \int_{0}^{\infty} e^{-\frac{\lambda}{2} l}\left(W_{0}^{\tau_{l}} \circ P_{0}^{(3, \mathrm{sym})}\right) d l
$$

In (1.1.35), we write $W_{0}^{\tau_{l}} \circ P_{0}^{(3, \text { sym })}$ for the image of the probability $W_{0}^{\tau_{l}} \otimes P_{0}^{(3, \text { sym })}$ by the concatenation operation $\circ$ :

$$
\circ: \Omega \times \Omega \longrightarrow \Omega
$$

defined by :

$$
X_{t}(\omega \circ \widetilde{\omega})=\left\{\begin{array}{rlr}
X_{t}(\omega) & \text { if } & t \leq \tau_{l}(\omega) \\
X_{t-\tau_{l}(\omega)}(\widetilde{\omega}) & \text { if } & t \geq \tau_{l}(\omega)
\end{array}\right.
$$

We note that $X_{\tau_{l}(\omega)}=0$. Such a notation has been used by Biane-Yor [B-Y] to whom we refer the reader. Let us note that formula (1.1.35) is nothing else but the translation of the results of point 2) of Theorem 1.1.5.

\subsubsection{Some remarkable properties of $\mathrm{W}$.}

We may now describe the measure $\mathbf{W}$ independently from any penalisation. We introduce :

$$
\begin{aligned}
& g:=\sup \left\{t ; X_{t}=0\right\}, \quad g_{a}:=\sup \left\{t ; X_{t}=a\right\} \\
& \sigma_{a, b}:=\sup \left\{t, X_{t} \in[a, b]\right\} \quad(a<b) \\
& \sigma_{a}:=\sup \left\{t, X_{t} \in[-a, a]\right\} \quad(a \geq 0)
\end{aligned}
$$


Theorem 1.1.6. The following identities hold:

1) $\mathbf{W}=\int_{0}^{\infty} d l\left(W_{0}^{\tau_{l}} \circ P_{0}^{(3, \mathrm{sym})}\right)$

2) i) For every $\left(\mathcal{F}_{t}, t \geq 0\right)$ stopping time $T$ and for any r.v. $\Gamma_{T}$ which is positive and $\mathcal{F}_{T}$ measurable:

$$
\mathbf{W}\left(\Gamma_{T} 1_{g<T} 1_{T<\infty}\right)=W\left(\Gamma_{T}\left|X_{T}\right| 1_{T<\infty}\right)
$$

ii) The law of $g$ under $\mathbf{W}$ is given by :

$$
\mathbf{W}(g \in d t)=\frac{d t}{\sqrt{2 \pi t}} \quad(t \geq 0)
$$

for every $t \geq 0$.

iii) Conditionally on $g=t$, the process $\left(X_{u}, u \leq g\right)$ under $\mathbf{W}$ is a Brownian bridge with length $t$. We denote by $\Pi_{0,0}^{(t)}$ the law of this bridge.

iv) $\quad \mathbf{W}=\int_{0}^{\infty} \frac{d t}{\sqrt{2 \pi t}}\left(\Pi_{0,0}^{(t)} \circ P_{0}^{(3, \mathrm{sym})}\right)$

v) For every previsible and positive process $\left(\phi_{s}, s \geq 0\right)$ we have:

$$
\mathbf{W}\left(\phi_{g}\right)=W\left(\int_{0}^{\infty} \phi_{s} d L_{s}\right)
$$

3) i) For every $\left(\mathcal{F}_{t}, t \geq 0\right)$ stopping time $T$, the law under $\mathbf{W}$ of $L_{\infty}-L_{T}$, on $T<\infty$ is given by:

$$
\begin{aligned}
\mathbf{W}\left(L_{\infty}-L_{T} \in d l, T<\infty\right) & =1_{[0, \infty]}(l) d l+W\left(\left|X_{T}\right| 1_{T<\infty}\right) \delta_{0}(d l) \\
& =1_{[0, \infty]}(l) d l+\mathbf{W}(g \leq T<\infty) \delta_{0}(d l)
\end{aligned}
$$

In particular, for $T=t$ :

$$
\mathbf{W}\left(L_{\infty}-L_{t} \in d l\right)=1_{[0, \infty[}(l) d l+\sqrt{\frac{2 t}{\pi}} \delta_{0}(d l)
$$

ii) For every $l>0$, conditionally on $L_{\infty}-L_{T}=l, T<\infty,\left(X_{u}, u \leq T\right)$ is a Brownian motion indexed by $[0, T]$

iii) The density of $\left(g, L_{\infty}\right)$ under $\mathbf{W}$ equals :

$$
f_{g, L_{\infty}}^{\mathbf{W}}(u, l)=\frac{l \exp \left(-\frac{l^{2}}{2 u}\right)}{\sqrt{2 \pi u^{3}}} 1_{[0, \infty[}(u) 1_{[0, \infty[}(l)
$$

\section{Remark 1.1.7.}

1) We deduce from formulae (1.1.43) and (1.1.17) that:

$$
\begin{aligned}
\varphi_{q}(0) & =\mathbf{W}\left(\exp \left(-\frac{1}{2} A_{\infty}^{(q)}\right)\right) \\
& =\int_{0}^{\infty} \frac{d t}{\sqrt{2 \pi t}} \Pi_{0,0}^{(t)}\left(\exp \left(-\frac{1}{2} A_{t}^{(q)}\right)\right) \cdot P_{0}^{(3, \text { sym })}\left(\exp \left(-\frac{1}{2} A_{\infty}^{(q)}\right)\right)
\end{aligned}
$$


2) It is proven in Biane-Yor ([B.Y], see also [Bia]) that :

$$
\int_{0}^{\infty} d l W_{0}^{\tau_{l}}=\int_{0}^{\infty} \frac{d t}{\sqrt{2 \pi t}} \Pi_{0,0}^{(t)}
$$

Thus,from this identity, we deduce easily that (1.1.40) implies (1.1.43).

3) Formula (1.1.41) (see also formulae (1.1.52), (1.1.54), (1.1.55), (1.1.56), (1.1.73)) yields a "representation" of the Brownian sub-martingale $\left(\left|X_{t}\right|, t \geq 0\right)$ in terms of the increasing process $\left(1_{g \leq t}, t \geq 0\right)$. (By a "representation" of a $\left(P,\left(\mathcal{F}_{t}, t \geq 0\right)\right)$ submartingale $\left(Z_{t}, t \geq 0\right)$, we mean a couple $\left(Q,\left(C_{t}, t \geq 0\right)\right)$ where $Q$ is a $\sigma$-finite measure and $\left(C_{t}, t \geq 0\right)$ is a increasing process such that, for every $\left.\Gamma_{t} \in b\left(\mathcal{F}_{t}\right): Q\left(\Gamma_{t} \cdot A_{t}\right)=E_{P}\left[\Gamma_{t} \cdot Z_{t}\right].\right)$ Here, the couple $\left(\mathbf{W}, 1_{g \leq t}\right)$ is a representation of the submartingale $\left(\left|X_{t}\right|, t \geq 0\right)$.

Before we prove Theorem 1.1.6, we present a slightly different version of it. We shall not prove this version, whose proof relies on close arguments to those we needed to obtain Theorem 1.1.6.

Theorem 1.1.8. Let $a \geq 0$; the following formulae hold:

1) For every $\left(\mathcal{F}_{t}, t \geq 0\right)$ stopping time $T$ and for every r.v. $\Gamma_{T}$ positive and $\mathcal{F}_{T}$ measurable:

$$
\mathbf{W}\left(\Gamma_{T} 1_{\left(\sigma_{a}<T<\infty\right)}=W\left(\Gamma_{T}\left(\left|X_{T}\right|-a\right)_{+} 1_{T<\infty}\right)\right.
$$

2) i) $\quad \mathbf{W}\left(\sigma_{a} \in d t\right)=\frac{e^{-\frac{a^{2}}{2 t}}}{\sqrt{2 \pi t}} d t \quad(t \geq 0)$

ii) $\quad \mathbf{W}=\int_{0}^{\infty} \frac{d t}{\sqrt{2 \pi t}} e^{-\frac{a^{2}}{2 t}} \frac{1}{2}\left(\Pi_{0, a}^{(t)} \circ P^{(a, 3)}+\Pi_{0,-a}^{(t)} \circ P^{(-a, 3)}\right)$

where $\Pi_{\alpha, \beta}^{(t)}$ denotes the law of the Brownian bridge of length $t$ starting from $\alpha$ and ending in $\beta$ and where $P^{(a, 3)}$ (resp. $\left.P^{(-a, 3)}\right)$ is the law of the process $\left(a+R_{t}, t \geq 0\right)$ (resp. $\left(-a-R_{t}\right.$, $t \geq 0)$ ) where $\left(R_{t}, t \geq 0\right)$ is a 3-dimensional Bessel process starting from 0. In particular, the law of $\left(X_{u}, u \leq \sigma_{a}\right)$, conditionally on $\sigma_{a}=t$ is $\frac{1}{2}\left(\Pi_{0, a}^{(t)}+\Pi_{0,-a}^{(t)}\right)$

iii) For every positive and previsible process $\left(\phi_{u}, u \geq 0\right)$, we have :

$$
\mathbf{W}\left(\phi_{\sigma_{a}}\right)=W\left(\int_{0}^{\infty} \phi_{u} d_{u}\left(\widetilde{L}_{u}^{a}\right)\right)
$$

with $\widetilde{L}_{u}^{a}:=\frac{1}{2}\left(L_{u}^{a}+L_{u}^{-a}\right)$.

We note that points 1) and 2) of Theorem 1.1.6 are particular cases of the corresponding ones in Theorem 1.1.8 when $a=0$. On the other hand, in the same spirit as for (1.1.48) we have, with the same kind of notation :

$$
\begin{gathered}
W\left(\Gamma_{T}\left(X_{T}-a\right)_{+} 1_{T<\infty}\right)=\mathbf{W}^{+}\left(\Gamma_{T} 1_{g_{a}<T<\infty}\right) \\
W\left(\Gamma_{T}\left(X_{T}-a\right)_{-} 1_{T<\infty}\right)=\mathbf{W}^{-}\left(\Gamma_{T} 1_{g_{a}<T<\infty}\right)
\end{gathered}
$$

where :

$$
\begin{aligned}
\mathbf{W}^{+} & =\frac{1}{2} \int_{0}^{\infty} \frac{d t}{\sqrt{2 \pi t}} \Pi_{0,0}^{(t)} \circ P_{0}^{(3)} \\
\mathbf{W}^{-} & =\frac{1}{2} \int_{0}^{\infty} \frac{d t}{\sqrt{2 \pi t}} \Pi_{0,0}^{(t)} \circ \widetilde{P}_{0}^{(3)}
\end{aligned}
$$


Adding (1.1.52) + and (1.1.52) - yields :

$$
W\left(\Gamma_{T}\left|X_{T}-a\right| 1_{T<\infty}\right)=\mathbf{W}\left(\Gamma_{T} 1_{g_{a}<T<\infty}\right)
$$

and also, with $a<b$ :

$$
W\left(\Gamma_{T}\left(\left(X_{T}-b\right)_{+}+\left(a-X_{T}\right)_{+}\right) 1_{T<\infty}\right)=\mathbf{W}\left(\Gamma_{T} 1_{\sigma_{a, b}<T<\infty}\right)
$$

and

$$
W\left(\Gamma_{T}\left(\left|X_{T}\right|-a\right){ }_{+} 1_{T<\infty}\right)=\mathbf{W}\left(\Gamma_{T} 1_{\sigma_{a}<T<\infty}\right) \quad(a \geq 0)
$$

Proof of Theorem 1.1.6.

Here is the plan of our proof. We shall use formula (1.1.16) with $q=\delta_{0}$ :

$$
\mathbf{W}=\varphi_{\delta_{0}}(0) e^{\frac{1}{2} L_{\infty}} \quad W_{\infty}^{\left(\delta_{0}\right)}=2 e^{\frac{1}{2} L_{\infty}} W_{\infty}^{\left(\delta_{0}\right)}
$$

as well as the properties of $W_{\infty}^{\left(\delta_{0}\right)}$ recalled in Theorem 1.1.5.

i) We prove (1.1.40) .

Let $F$ and $G$ be two positive functionals. We have, from (1.1.57) :

$$
\begin{aligned}
\mathbf{W}( & \left.F\left(X_{s}, s \leq g\right) \cdot G\left(X_{g+s}, s \geq 0\right)\right) \\
= & 2 W_{\infty}^{\left(\delta_{0}\right)}\left(e^{\frac{1}{2} L_{\infty}} F\left(X_{s}, s \leq g\right) G\left(X_{g+s}, s \geq 0\right)\right) \\
= & 2 W_{\infty}^{\left(\delta_{0}\right)}\left(e^{\frac{1}{2} L_{g}} F\left(X_{s}, s \leq g\right) G\left(X_{g+s}, s \geq 0\right)\right) \\
& \left(\text { since } L_{\infty}=L_{g}\right) \\
= & 2 W_{\infty}^{\left(\delta_{0}\right)}\left(e^{\frac{1}{2} L_{g}} F\left(X_{s}, s \leq g\right)\right) \cdot P_{0}^{(3, \mathrm{sym})}\left(G\left(X_{s}, s \geq 0\right)\right) \\
& (\text { from }(1.1 .35)) \\
= & \left(2 \int_{0}^{\infty} W_{\infty}^{\left(\delta_{0}\right)}\left(e^{\frac{1}{2} L_{g}} F\left(X_{s}, s \leq g\right) \mid L_{g}=l\right) \frac{1}{2} e^{-\frac{l}{2}} d l\right) \cdot P_{0}^{(3, \mathrm{sym})}\left(G\left(X_{s}, s \geq 0\right)\right) \\
= & \left(2 \int_{0}^{\infty} e^{\frac{l}{2}} W\left(F\left(X_{s}, s \leq \tau_{l}\right) \frac{1}{2} e^{-\frac{l}{2}} d l\right)\right) \cdot P_{0}^{(3, \mathrm{sym})}\left(G\left(X_{s}, s \geq 0\right)\right) \\
= & \int_{0}^{\infty} d l\left(W_{0}^{\tau_{l}} \circ P_{0}^{(3, \mathrm{sym})}\right)\left(F\left(X_{s}, s \leq g\right) \cdot G\left(X_{g+s}, s \geq 0\right)\right)
\end{aligned}
$$

from point 2, iv) of Theorem 1.1.5.

ii) We now prove (1.1.41).

For this purpose, we apply formula (1.1.16) with $q=\lambda \delta_{0}$. Thus :

$$
A_{t}^{(q)}=\lambda L_{t} \quad \text { and, from (1.1.30), } \quad \varphi_{\lambda \delta_{0}}(x)=\frac{2}{\lambda}+|x| .
$$

Thus, from (1.1.16), (1.1.17), (1.1.18) and Doob's optional stopping Theorem :

$$
\begin{aligned}
W\left(\Gamma_{T}\left(\frac{2}{\lambda}+\left|X_{T}\right|\right) 1_{T<\infty}\right) & =\frac{2}{\lambda} W_{\infty}^{\left(\lambda \delta_{0}\right)}\left(e^{\frac{\lambda}{2} L_{T}} \Gamma_{T} 1_{T<\infty}\right) \\
& =\mathbf{W}\left(\Gamma_{T} 1_{g \leq T<\infty}\right)+\mathbf{W}\left(\Gamma_{T} 1_{g>T} e^{-\frac{\lambda}{2}\left(L_{\infty}-L_{T}\right)}\right)
\end{aligned}
$$


We then let $\lambda \longrightarrow \infty$ in (1.1.58) and note that $L_{\infty}-L_{T}>0$ on $g>T$. The monotone convergence Theorem implies :

$$
W\left(\Gamma_{T}\left|X_{T}\right| 1_{T<\infty}\right)=\mathbf{W}\left(\Gamma_{T} 1_{g \leq T<\infty}\right)
$$

This is precisely relation (1.1.41). Relation (1.1.42) in an easy consequence of (1.1.41).

iii) We prove (1.1.45) and (1.1.46).

We note that (1.1.41) and (1.1.58) imply :

$$
\begin{aligned}
\frac{2}{\lambda} W\left(\Gamma_{T} 1_{T<\infty}\right) & =\mathbf{W}\left(\Gamma_{T} 1_{g>T} \exp \left(-\frac{\lambda}{2}\left(L_{\infty}-L_{T}\right)\right)\right) \\
& =W\left(\Gamma_{T} 1_{T<\infty}\right)\left(\int_{0}^{\infty} e^{-\frac{\lambda}{2} l} d l\right)
\end{aligned}
$$

Thus, by injectivity of the Laplace transform, for every function $\psi: \mathbb{R}_{+} \longrightarrow \mathbb{R}_{+}$Borel and integrable :

$$
W\left(\Gamma_{T} 1_{T<\infty}\right)\left(\int_{0}^{\infty} \psi(l) d l\right)=\mathbf{W}\left(\Gamma_{T} \psi\left(L_{\infty}-L_{T}\right) 1_{g>T}\right)
$$

and

$$
W\left(\left|X_{T}\right| 1_{T<\infty}\right)=\mathbf{W}(g \leq T<\infty)=\mathbf{W}\left(L_{\infty}-L_{T}=0, T<\infty\right)
$$

In other terms, we have :

$$
\mathbf{W}\left(L_{\infty}-L_{T} \in d l, T<\infty\right)=1_{[0, \infty[}(l) d l+W\left(\left|X_{T}\right| 1_{T<\infty}\right) \delta_{0}(d l)
$$

and conditionally on $L_{\infty}-L_{T}=l(l>0), \quad\left(X_{u}, u \leq T\right)$ is a Brownian motion indexed by $[0, T]$. This is (1.1.45) and (1.1.46).

iv) We now prove point 2, iii) of Theorem 1.1.6.

For this purpose, we write (1.1.41), choosing for $\Gamma_{t}$ a r.v. of the form $\Phi_{g^{(t)}}$, where $\left(\Phi_{u}, u \geq 0\right)$ is a previsible positive process, and where $g^{(t)}:=\sup \left\{s \leq t, X_{s}=0\right\}$.

The LHS of (1.1.41) becomes :

$$
\begin{aligned}
W\left(\left|X_{t}\right| \Phi_{g^{(t)}}\right)= & W\left(\int_{0}^{t} \Phi_{s} d L_{s}\right) \\
& (\text { from the balayage formula (cf }[\mathrm{R}, \mathrm{Y}], \text { Chap. VI, p. 260)) } \\
= & \int_{0}^{t} W\left(\Phi_{s} \mid X_{s}=0\right) W\left(d L_{s}\right) \\
= & \int_{0}^{t} W\left(\Phi_{s} \mid X_{s}=0\right) \frac{d s}{\sqrt{2 \pi s}} \\
& \left(\text { since } W\left(L_{s}\right)=W\left(\left|X_{s}\right|\right)=\sqrt{\frac{2 s}{\pi}}\right)
\end{aligned}
$$


The RHS of (1.1.41) writes :

$$
\begin{aligned}
\mathbf{W}\left(\Phi_{g^{(t)}} 1_{g \leq t}\right)= & \mathbf{W}\left(\Phi_{g} 1_{g \leq t}\right) \\
& \left(\text { since } g=g^{(t)} \text { on the set }\{g \leq t\}\right) \\
= & \int_{0}^{t} \mathbf{W}\left(\Phi_{g} \mid g=s\right) \frac{d s}{\sqrt{2 \pi s}} \\
& \text { from }(1.1 .42) . \text { Thus : } \\
\int_{0}^{t} W\left(\Phi_{s} \mid X_{s}=0\right) \frac{d s}{\sqrt{2 \pi s}}= & \int_{0}^{t} \mathbf{W}\left(\Phi_{g} \mid g=s\right) \frac{d s}{\sqrt{2 \pi s}}
\end{aligned}
$$

This relation implies $\mathbf{W}\left(\Phi_{s} \mid g=s\right)=W\left(\Phi_{s} \mid X_{s}=0\right)$, i.e. point 2, iii) of Theorem 1.1.6. We also note that we deduce from the equality between (1.1.62) and (1.1.63) :

$$
W\left(\int_{0}^{t} \Phi_{s} d L_{s}\right)=\int_{0}^{t} \mathbf{W}\left(\Phi_{g} \mid g=s\right) \frac{d s}{\sqrt{2 \pi s}}
$$

that :

$$
\begin{aligned}
W\left(\int_{0}^{\infty} \Phi_{s} d L_{s}\right) & =\int_{0}^{\infty} \mathbf{W}\left(\Phi_{g} \mid g=s\right) \mathbf{W}(g \in d s) \\
& =\mathbf{W}\left(\Phi_{g}\right)
\end{aligned}
$$

i.e. point 2,v) of Theorem 1.1.6.

v) We now prove point 2, iv) of Theorem 1.1.6.

We obtain, with the help of (1.1.57), for two positive functionals $F$ and $G$ :

$$
\begin{aligned}
& \mathbf{W}\left(F\left(X_{s}, s \leq g\right) G\left(X_{g+s}, s \geq 0\right)\right) \\
& \quad=2 W_{\infty}^{\left(\delta_{0}\right)}\left(F\left(X_{s}, s \leq g\right) e^{\frac{1}{2} L_{g}} G\left(X_{g+s, s \geq 0}\right)\right) \\
& =2 W_{\infty}^{\left(\delta_{0}\right)}\left(F\left(X_{s}, s \leq g\right) e^{\frac{1}{2} L_{g}}\right) P_{0}^{(3, \mathrm{sym})}\left(G\left(X_{s}, s \geq 0\right)\right)
\end{aligned}
$$

(from point 2 ii) and 2 iii) of Theorem 1.1.6)

$$
=\mathbf{W}\left(F\left(X_{s}, s \leq g\right)\right) \cdot P_{0}^{(3, \mathrm{sym})}\left(G\left(X_{s}, s \geq 0\right)\right)
$$

(using once again (1.1.57))

$$
=\left(\int_{0}^{\infty} \mathbf{W}\left(F\left(X_{s}, s \leq g\right) \mid g=t\right) \frac{d t}{\sqrt{2 \pi t}}\right) \cdot P_{0}^{(3, \text { sym })}\left(G\left(X_{s}, s \geq 0\right)\right)
$$

(from (1.1.42))

$$
=\int_{0}^{\infty} \frac{d t}{\sqrt{2 \pi t}} \Pi_{0,0}^{(t)}\left(F\left(X_{s}, s \leq t\right)\right) \cdot P_{0}^{(3, \mathrm{sym})}\left(G\left(X_{s}, s \geq 0\right)\right)
$$

(from point $2 \mathrm{iv}$ ) of Theorem 1.1.6)

$$
=\int_{0}^{\infty} \frac{d t}{\sqrt{2 \pi t}}\left(\Pi_{0,0}^{(t)} \circ P_{0}^{(3, \mathrm{sym})}\right)\left(F\left(X_{s}, s \leq g\right) G\left(X_{g+s}, s \geq 0\right)\right) .
$$

vi) Formula (1.1.47) is a consequence of (1.1.42), (1.1.43) and the fact that: Under $\Pi_{0,0}^{(t)}, L_{t}$ is distributed as $\sqrt{2 t \mathfrak{e}}$, where $\mathfrak{e}$ is a standard exponential r.v.

Remark 1.1.9.

1) We have, from (1.1.16) and Theorem 1.1.5 :

$$
\frac{\lambda}{2} e^{-\frac{\lambda}{2} L_{\infty}} \cdot \mathbf{W}=W_{\infty}^{\left(\lambda \delta_{0}\right)}
$$


But, from Theorem 1.1.1, under $W_{\infty}^{\left(\lambda \delta_{0}\right)}$ :

$$
X_{t}=B_{t}+\int_{0}^{t} \frac{\operatorname{sgn} X_{s}}{\frac{2}{\lambda}+\left|X_{s}\right|} d s
$$

Hence (see [RY, M], Chap. 4) : $W_{\infty}^{\left(\lambda \delta_{0}\right)} \underset{\lambda \rightarrow \infty}{\longrightarrow} P_{0}^{(3, \text { sym })}$.

Thus $\frac{\lambda}{2}\left(e^{-\frac{\lambda}{2} L_{\infty}}\right) \mathbf{W} \underset{\lambda \rightarrow \infty}{\longrightarrow} P_{0}^{(3, \mathrm{sym})}$

This convergence holds in the sense of weak convergence with respect to the topology of uniform convergence on compacts in $\mathcal{C}([0, \infty[\rightarrow \mathbb{R})$.

2) Formula (1.1.41) may be proven in a different manner than by the way we have indicated. Indeed, from (1.1.57)

$$
\begin{aligned}
\mathbf{W}\left(\Gamma_{t} 1_{g \leq t}\right)= & 2 W_{\infty}^{\left(\delta_{0}\right)}\left(\Gamma_{t} 1_{g \leq t} e^{\frac{1}{2} L_{\infty}}\right) \\
= & 2 W_{\infty}^{\left(\delta_{0}\right)}\left(\Gamma_{t} 1_{g \leq t} e^{\frac{1}{2} L_{t}}\right) \\
& \left(\text { since } L_{\infty}=L_{t} \text { on the set }(g \leq t)\right) \\
= & 2 W_{\infty}^{\left(\delta_{0}\right)}\left(\Gamma_{t} e^{\frac{1}{2} L_{t}} W_{\infty}^{\left(\delta_{0}\right)}\left(1_{g \leq t} \mid \mathcal{F}_{t}\right)\right.
\end{aligned}
$$

But

$$
\begin{aligned}
W_{\infty}^{\left(\delta_{0}\right)}\left(1_{g \leq t} \mid \mathcal{F}_{t}\right) & =W_{\infty}^{\left(\delta_{0}\right)}\left(T_{0} \circ \theta_{t}=\infty \mid \mathcal{F}_{t}\right) \\
& =W_{X_{t}, \infty}^{\left(\delta_{0}\right)}\left(T_{0}=\infty\right)
\end{aligned}
$$

with $T_{0}=\inf \left\{t \geq 0 ; X_{t}=0\right\}$, by the Markov property. But, from (1.1.14), the scale function of the process $\left(X_{t}, t \geq 0\right)$ under $\left(W_{x, \infty}^{\left(\delta_{0}\right)}, x \in \mathbb{R}\right)$ equals :

$$
\gamma_{\delta_{0}}(x)=\frac{x}{2(2+|x|)}
$$

We deduce from (1.1.69) :

$$
W_{x, \infty}^{\left(\delta_{0}\right)}\left(T_{0}=\infty\right)=\frac{|x|}{2+|x|}
$$

Plugging (1.1.70) and (1.1.68) in (1.1.67), we obtain :

$$
\begin{aligned}
\mathbf{W}\left(\Gamma_{t} 1_{g \leq t}\right) & =2 W_{\infty}^{\left(\delta_{0}\right)}\left(\Gamma_{t} e^{\frac{1}{2} L_{t}} \frac{\left|X_{t}\right|}{2+\left|X_{t}\right|}\right) \\
& =2 W\left(\Gamma_{t} e^{\frac{1}{2} L_{t}} \frac{\left|X_{t}\right|}{2+\left|X_{t}\right|} \frac{2+\left|X_{t}\right|}{2} e^{-\frac{1}{2} L_{t}}\right) \\
& =W\left(\Gamma_{t}\left|X_{t}\right|\right)
\end{aligned}
$$

Formulae (1.1.51), (1.1.54), (1.1.55), (1.1.56) may be proven following the same arguments.

3) Let $q \in \mathcal{I}$ such that the convex hull of its support equals the interval $[a, b](a \leq b)$. From (1.1.7) and (1.1.6) we have :

$$
\begin{aligned}
W\left(\varphi_{\lambda q}\left(X_{t}\right) \cdot \Gamma_{t}\right) & =\varphi_{\lambda q}(0) W_{\infty}^{(\lambda q)}\left(\Gamma_{t} e^{-\frac{\lambda}{2} A_{t}^{(q)}}\right) \\
& =\mathbf{W}\left(\Gamma_{t} e^{-\frac{\lambda}{2}\left(A_{\infty}^{(q)}-A_{t}^{q}\right)}\right) \\
& =\mathbf{W}\left(\Gamma_{t} 1_{\sigma_{a b} \leq t}\right)+\mathbf{W}\left(\Gamma_{t} e^{-\frac{\lambda}{2}\left(A_{\infty}^{(q)}-A_{t}^{q}\right)} 1_{\sigma_{a b}>t}\right)
\end{aligned}
$$


On the other hand, we have proven in [RY, IX] (see also [RY, M], Chap. 2) that there exists, for every $x \in \mathbb{R}$, a positive and $\sigma$-finite measure $\nu_{x}^{(q)}$ such that :

$$
\int_{0}^{\infty} e^{-\frac{\lambda y}{2}} \nu_{x}^{(q)}(d y)=\varphi_{\lambda q}(x)
$$

It remains to let $t \rightarrow \infty$ in $(1.1 .72)$ to obtain, since $A_{\infty}^{(q)}-A_{t}^{(q)}>0$ on the set $\left(\begin{array}{lll}\sigma_{a} & b & >\end{array}\right)$ :

$$
W\left(\Gamma_{t} \nu_{X_{t}}^{(q)}(\{0\})=\mathbf{W}\left(\Gamma_{t} 1_{\sigma_{a b} \leq t}\right)\right.
$$

Hence, $\nu_{x}^{(q)}(\{0\})$ depends only on $\operatorname{supp}(q)$ and $\left(\nu_{X_{t}}^{(q)}(\{0\}), t \geq 0\right)$ is a sub-martingale. Formula (1.1.56) (with $T=t$ ) is a particular case of (1.1.73), since :

$$
\nu_{x}^{\left(\delta_{a}+\delta_{b}\right)}(\{0\})=(x-b)_{+}+(a-x)_{+}
$$

(see $[R Y, I X])$.

\subsubsection{Another approach to Theorem 1.1.6.}

Let, for $q \in \mathcal{I}$, the probability $W_{\infty}^{(q)}$ be defined by (1.1.18). Then :

$$
\left.W_{\infty}^{(q)}\right|_{\mathcal{F}_{t}}=\frac{\varphi_{q}\left(X_{t}\right)}{\varphi_{q}(0)} e^{-\frac{1}{2} A_{t}^{(q)}} \cdot W_{\left.\right|_{\mathcal{F}_{t}}}
$$

In Theorem 1.1.2, we have defined the measure $\mathbf{W}$ from the formula :

$$
\mathbf{W}=\varphi_{q}(0) e^{\frac{1}{2} A_{\infty}^{(q)}} W_{\infty}^{(q)}
$$

then, by showing that :

$$
\mathbf{W}=\int_{0}^{\infty} \frac{d t}{\sqrt{2 \pi t}}\left(\Pi_{0,0}^{(t)} \circ P_{0}^{(3, \mathrm{sym})}\right)
$$

(cf Theorem 1.1.6, relation (1.1.43)). We now "forget" our previous results and proceed in a reverse way. For this purpose, we define, for the time being, the measure :

$$
\widetilde{\mathbf{W}}=\int_{0}^{\infty} \frac{d t}{\sqrt{2 \pi t}}\left(\Pi_{0,0}^{(t)} \circ P_{0}^{(3, \mathrm{sym})}\right)
$$

We shall show that, for every $q \in \mathcal{I}$ :

$$
\frac{1}{\varphi_{q}(0)} e^{-\frac{1}{2} A_{\infty}^{(q)}} \cdot \widetilde{\mathbf{W}}=W_{\infty}^{(q)}
$$

\section{Theorem 1.1.10.}

Let $\widetilde{\mathbf{W}}$ be defined by (1.1.78) and $W_{\infty}^{(q)}$ be defined by (1.1.75). Then, for every $q \in \mathcal{I}$ :

$$
\frac{1}{\varphi_{q}(0)} e^{-\frac{1}{2} A_{\infty}^{(q)}} \cdot \widetilde{\mathbf{W}}=W_{\infty}^{(q)}
$$

Proof of Theorem 1.1.10. 
We compute the value of $W_{\infty}^{(q)}$ when integrating the following general class of functionals which are $\mathcal{F}_{t}$-measurable and positive :

$$
F\left(X_{u}, u \leq g^{(t)}\right) \cdot G\left(X_{g^{(t)}+u}, u \leq t-g^{(t)}\right)
$$

We have :

$$
\begin{aligned}
& W_{\infty}^{(q)}\left(F\left(X_{u}, u \leq g^{(t)}\right) G\left(X_{g^{(t)}+u} ; u \leq t-g^{(t)}\right)\right) \\
& \quad=\frac{1}{\varphi_{q}(0)} W\left[F\left(X_{u}, u \leq g^{(t)}\right) G\left(X_{g^{(t)}+u} ; v \leq t-g^{(t)}\right) \exp \left(-\frac{1}{2} A_{t}^{(q)}\right) \varphi_{q}\left(X_{t}\right)\right]
\end{aligned}
$$

$($ from $(1.1 .75))$

$$
\begin{gathered}
=\frac{1}{\varphi_{q}(0)} W\left[F\left(X_{u}, u \leq g^{(t)}\right) \exp \left(-\frac{1}{2} A_{g^{(t)}}^{(q)}\right) \cdot G\left(X_{g^{(t)}+u}, u \leq t-g^{(t)}\right)\right. \\
\left.\cdot \varphi_{q}\left(X_{t}\right) \exp \left(-\frac{1}{2}\left(A_{t}^{(q)}-A_{g^{(t)}}^{(q)}\right)\right)\right]
\end{gathered}
$$

We now consider the probability $W$ restricted to $\mathcal{F}_{t}$, denoted as $W^{(t)}$, which we disintegrate with respect to the law of $g^{(t)}$ :

$$
\begin{aligned}
W^{(t)}= & \int_{0}^{t} \frac{d u}{\pi \sqrt{u(t-u)}}\left(\Pi_{0,0}^{(u)} \circ M^{(t-u, \text { sym })}\right) \\
& \text { with : } \\
W\left(g^{(t)} \in d u\right)= & \frac{d u}{\pi \sqrt{u(t-u)}} \quad u \leq t
\end{aligned}
$$

and where $\Pi_{0,0}^{(u)}$ denotes the law of the Brownian bridge with length $u$ and $M^{(t, \text { sym })}$ is the law of a symmetric Brownian meander of length $t$. Thus, (1.1.82) becomes :

$$
\begin{aligned}
& W_{\infty}^{(q)}\left[F\left(X_{u}, u \leq g^{(t)}\right) G\left(X_{g^{(t)}+v}, v \leq t-g^{(t)}\right)\right] \\
& \left.=\frac{1}{\varphi_{q}(0)} \int_{0}^{t} \frac{d u}{\pi \sqrt{u(t-u)}} \Pi_{0,0}^{(u)}\left(F\left(X_{v}, v \leq u\right)\right) e^{-\frac{1}{2} A_{u}^{(q)}}\right) \\
& \quad \cdot M^{(t-u, \operatorname{sym})}\left(\varphi_{q}\left(X_{t-u}\right) e^{-\frac{1}{2} A_{t-u}^{(q)}} \cdot G\left(X_{l}, l \leq t-u\right)\right)
\end{aligned}
$$

Using now Imhof's relation (see [RY, M], Chap. 1, Item $G$ ) :

$$
M^{(t, \mathrm{sym})}=\left.\sqrt{\frac{\pi t}{2}} \frac{1}{\left|X_{t}\right|} P_{0}^{(3, \mathrm{sym})}\right|_{\mathcal{F}_{t}}
$$

we obtain :

$$
\begin{aligned}
& W_{\infty}^{(q)}\left[F\left(X_{u}, u \leq g^{(t)}\right) G\left(X_{g^{(t)}}+v, v \leq t-g^{(t)}\right)\right] \\
& =\frac{1}{\varphi_{q}(0)} \int_{0}^{t} \frac{d u}{\pi \sqrt{u(t-u)}} \Pi_{0,0}^{(u)}\left(F\left(X_{v} v \leq u\right) e^{-\frac{1}{2} A_{u}^{(q)}}\right) \\
& \quad \cdot P_{0}^{(3, \mathrm{sym})}\left(\varphi_{q}\left(X_{t-u}\right) \frac{G\left(X_{l}, l \leq t-u\right)}{\left|X_{t-u}\right|} \sqrt{\frac{\pi}{2}(t-u)} e^{-\frac{1}{2} A_{t-u}^{(q)}}\right)
\end{aligned}
$$


We observe that the factor $\sqrt{t-u}$ simplifies on the RHS of (1.1.84). We then let $t \longrightarrow \infty$ in (1.1.84), by using the fact that $\varphi_{q}(x) \underset{|x| \rightarrow \infty}{\sim}|x|$. We obtain, since $g^{(t)} \underset{t \rightarrow \infty}{\longrightarrow} g$ a.s. under $W_{\infty}^{(q)}$ (cf Theorem 1.1.1) :

$$
\begin{aligned}
& W_{\infty}^{(q)}\left(F\left(X_{u}, u \leq g\right) \cdot G\left(X_{g+v}, v \geq 0\right)\right) \\
& =\frac{1}{\varphi_{q}(0)}\left(\int_{0}^{\infty} \frac{d u}{\sqrt{2 \pi u}} \Pi_{0,0}^{(u)}\left(F\left(X_{v}, v \leq u\right) e^{-\frac{1}{2} A_{u}^{(q)}}\right) \cdot P_{0}^{(3, \mathrm{sym})}\left(G\left(X_{l}, l \geq 0\right) e^{-\frac{1}{2} A_{\infty}^{(q)}}\right)\right. \\
& =\frac{1}{\varphi_{q}(0)} \widetilde{\mathbf{W}}\left(e^{-\frac{1}{2} A_{\infty}^{(q)}} F\left(X_{u}, u \leq g\right) G\left(X_{g+l}, l \geq 0\right)\right)
\end{aligned}
$$

This is the statement of Theorem 1.1.10.

1.1.7 Relations between $W$ and other penalisations (than the Feynman-Kac ones). We have shown - this is Theorem 1.1.2 - that for every $q \in \mathcal{I}$ :

$$
\begin{aligned}
\mathbf{W} & =\varphi_{q}(0) \exp \left(\frac{1}{2} A_{\infty}^{(q)}\right) \cdot W_{\infty}^{(q)} \\
& =\mathbf{W}\left(\exp \left(-\frac{1}{2} A_{\infty}^{(q)}\right)\right) \cdot \exp \left(\frac{1}{2} A_{\infty}^{(q)}\right) \cdot W_{\infty}^{(q)}
\end{aligned}
$$

Of course, this formula is very much linked with the penalisation of the Wiener measure by the multiplicative functional $\left(F_{t}=\exp \left(-\frac{1}{2} A_{t}^{(q)}\right), t \geq 0\right)$. Here, we shall prove that formulae analogous to (1.1.85) are true for other penalisations than these Feynman-Kac ones. We now fix some notations :

$$
\begin{gathered}
S_{t}:=\sup _{s \leq t} X_{s}, \quad I_{t}:=\inf _{s \leq t} X_{s} \\
\Gamma_{+}:=\left\{\omega \in \Omega ; \lim _{t \rightarrow \infty} X_{t}(\omega)=+\infty\right\}, \Gamma_{-}:=\left\{\omega \in \Omega, \lim _{t \rightarrow \infty} X_{t}(\omega)=-\infty\right\} \\
\mathbf{W}^{+}:=1_{\Gamma_{+}} \cdot \mathbf{W}, \quad \mathbf{W}^{-}:=1_{\Gamma_{-}} \cdot \mathbf{W} \\
\theta_{+}:=\sup \left\{t ; S_{t}<S_{\infty}\right\}, \quad \theta_{-}:=\sup \left\{t ; I_{t}>I_{\infty}\right\}
\end{gathered}
$$

Let $\psi_{+}$(resp. $\psi_{-}$) a Borel and integrable function from $\mathbb{R}_{+}$to $\mathbb{R}_{+}$(resp. from $\mathbb{R}_{-}$to $\mathbb{R}_{+}$). We denote by $\left(M_{s}^{\psi_{+}(S)}, s \geq 0\right)$ (resp. $\left(M_{s}^{\psi_{-}(I)}, s \geq 0\right)$ ) the Azéma-Yor martingale defined by :

$$
\begin{aligned}
& M_{s}^{\psi_{+}(S)}:=\frac{1}{\left(\int_{0}^{\infty} \psi_{+}(y) d y\right)}\left(\psi_{+}\left(S_{s}\right)\left(S_{s}-X_{s}\right)+\int_{S_{s}}^{\infty} \psi_{+}(y) d y\right) \\
& M_{s}^{\psi_{-}(I)}:=\frac{1}{\left(\int_{-\infty}^{0} \psi_{-}(y) d y\right)}\left(\psi_{-}\left(I_{s}\right)\left(X_{s}-I_{s}\right)+\int_{-\infty}^{I_{s}} \psi_{-}(y) d y\right)
\end{aligned}
$$

Let $W_{\infty}^{\psi_{+}{ }^{(S)}}\left(\operatorname{resp} W_{\infty}^{\psi_{-}(I)}\right)$ denote the probability on $\left(\Omega, \mathcal{F}_{\infty}\right)$ characterized by :

$$
W_{\infty}^{\psi_{+}(S)}{ }_{\left.\right|_{\mathcal{F}_{t}}}=M_{t}^{\psi_{+}(S)} \cdot W_{\left.\right|_{\mathcal{F}_{t}}},\left.\quad W_{\infty}^{\psi_{-}(I)} \cdot\right|_{\mathcal{F}_{t}}=M_{t}^{\psi{ }^{(I)}} \cdot W_{\left.\right|_{\mathcal{F}_{t}}}
$$

(see [RVY, II] for more informations about these probabilities). 
The analogue of formulae (1.1.85) and (1.1.41) is here :

Theorem 1.1.11. Let $\psi_{+}, \psi_{-}$as above, with $\psi_{+}(\infty)=\psi_{-}(\infty)=0$.

1) $\quad \mathbf{W}^{-}=\mathbf{W}\left(\psi_{+}\left(S_{\infty}\right)\right) \cdot \frac{1}{\psi_{+}\left(S_{\infty}\right)} \cdot W_{\infty}^{\psi_{+}(S)}$

$$
\mathbf{W}^{+}=\mathbf{W}\left(\psi_{-}\left(I_{\infty}\right)\right) \frac{1}{\psi_{-}\left(I_{\infty}\right)} W_{\infty}^{\psi_{-}(I)}
$$

2) For every $t \geq 0$ and $\Gamma_{t} \in b_{+}\left(\mathcal{F}_{t}\right)$ :

$$
\begin{aligned}
& W\left(\Gamma_{t}\left(S_{t}-X_{t}\right)\right)=\mathbf{W}^{-}\left(\Gamma_{t} 1_{\theta_{+} \leq t}\right) \\
& W\left(\Gamma_{t}\left(X_{t}-I_{t}\right)\right)=\mathbf{W}^{+}\left(\Gamma_{t} 1_{\theta_{-} \leq t}\right)
\end{aligned}
$$

\section{Proof of Theorem 1.1.11.}

i) We have, from (1.1.85), for $q \in \mathcal{I}$, and $\Gamma_{t} \in b_{+}\left(\mathcal{F}_{t}\right)$ :

$$
\begin{aligned}
& \mathbf{W}\left(e^{-\frac{1}{2} A_{\infty}^{(q)}} \cdot \Gamma_{t}\right) \\
&=\varphi_{q}(0) W_{\infty}^{(q)}\left(\Gamma_{u}\right) \\
&= W\left(\Gamma_{t} \varphi_{q}\left(X_{t}\right) e^{-\frac{1}{2} A_{t}^{(q)}}\right) \\
&(\text { from }(1.1 .7) \text { and }(1.1 .8)) \\
&=\left(\int_{0}^{\infty} \psi(y) d y\right) \cdot W_{\infty}^{\psi(S)}\left(\Gamma_{u} \frac{\varphi_{q}\left(X_{t}\right) e^{-\frac{1}{2} A_{t}^{(q)}}}{\psi\left(S_{t}\right)\left(S_{t}-X_{t}\right)+\int_{S_{t}}^{\infty} \psi(y) d y}\right)
\end{aligned}
$$

from (1.1.92) and (1.1.90), and we write, to simplify, $\psi$ for $\psi_{+}$. Formula (1.1.97) being true for every $\Gamma_{t} \in b_{+}\left(\mathcal{F}_{t}\right)$, we may take $\Gamma_{t}=\Gamma_{u} 1_{\psi\left(S_{t}\right)>a} \cdot 1_{S_{t}-X_{t}>b\left|X_{t}\right|} \cdot 1_{\int_{S_{t}}}^{\infty} \psi(y) d y>c$ with $0<b<1$, $a, c>0$ for any $\Gamma_{u} \in \mathcal{F}_{u}, u \leq t$. We obtain thus :

$$
\begin{aligned}
& \mathbf{W}\left[\Gamma_{u} e^{-\frac{1}{2} A_{\infty}^{(q)}} 1_{\psi\left(S_{t}\right)>a} 1_{S_{t}-X_{t}>b\left|X_{t}\right|} 1_{\int_{S_{t}}^{\infty} \psi(y) d y>c}\right] \\
& =\left(\int_{0}^{\infty} \psi(y) d y\right) \cdot W_{\infty}^{\psi(S)}\left[\Gamma_{u} \frac{\varphi_{q}\left(X_{t}\right) e^{-\frac{1}{2} A_{t}^{(q)}}}{\psi\left(S_{t}\right)\left(S_{t}-X_{t}\right)+\int_{S_{t}}^{\infty} \psi(y) d y} 1_{\psi\left(S_{t}\right)>a}\right. \\
& \left.1_{\left.S_{t}-X_{t}>b \mid X_{t}\right]} 1_{\int_{S_{t}}^{\infty} \psi(y) d y>c}\right]
\end{aligned}
$$

We shall now let $t \rightarrow \infty$ in (1.1.98) with $u$ being fixed. On the LHS, we have :

$$
\begin{array}{ll}
\mathbf{W}^{+} \text {a.s. } & 1_{\psi\left(S_{t}\right)>a} \underset{t \rightarrow \infty}{\longrightarrow} 0 \quad\left(\text { since } S_{t} \longrightarrow+\infty \text { and } \psi\left(S_{t}\right) \underset{t \rightarrow \infty}{\longrightarrow} 0\right) \\
\mathbf{W}^{-} \text {a.s. } & 1_{\psi\left(S_{t}\right)>a} \underset{t \rightarrow \infty}{\longrightarrow} 1_{\psi\left(S_{\infty}\right)>a} \\
& 1_{\left.S_{t}-X_{t}>b \mid X_{t}\right]} \underset{t \rightarrow \infty}{\longrightarrow} \\
& 1_{\int_{S_{t}}^{\infty} \psi(y) d y>c}^{\longrightarrow} 1_{\int_{S_{\infty}}^{\infty} \psi(y) d y>c}
\end{array}
$$

Thus, from Lebesgue's dominated convergence Theorem, the LHS of (1.1.98) converges, as $t \rightarrow \infty$, towards $L$, with :

$$
L=\mathbf{W}\left(\Gamma_{u} 1_{\Gamma_{-}} e^{-\frac{1}{2} A_{\infty}^{q}} 1_{\psi\left(S_{\infty}\right)>a} 1_{\int_{S_{\infty}}^{\infty} \psi(y) d y>c}\right\}
$$


We now consider the RHS of (1.1.98). On the set :

$$
\left(\psi\left(S_{t}\right)>a\right) \cap\left(S_{t}-X_{t}>b\left|X_{t}\right|\right) \cap\left(\int_{S_{t}}^{\infty} \psi(y) d y>c\right),
$$

we have :

$$
\frac{\varphi_{q}\left(X_{t}\right)}{\psi\left(S_{t}\right)\left(S_{t}-X_{t}\right)+\int_{S_{t}}^{\infty} \psi(y) d y} \leq \frac{d+\left|X_{t}\right|}{a b\left|X_{t}\right|+c} \leq k
$$

since $\left|\varphi_{q}(x)\right| \leq d+|x|$; thus, we may apply the dominated convergence Theorem to obtain, since under $W_{\infty}^{\psi(S)}$ (see [RVY, II]) : $X_{t} \underset{t \rightarrow \infty}{\longrightarrow}-\infty$ and $S_{t} \underset{t \rightarrow \infty}{\longrightarrow} S_{\infty}$ a.s., the convergence of the RHS of (1.1.98) to $R$, with :

$$
R=\left(\int_{0}^{\infty} \psi(y) d y\right) \cdot W_{\infty}^{\psi(S)}\left(\frac{1}{\psi\left(S_{\infty}\right)} e^{-\frac{1}{2} A_{\infty}^{(q)}} 1_{\psi\left(S_{\infty}\right)>a} 1_{\int_{S_{\infty}}^{\infty} \psi(y) d y>c}\right)
$$

(since $\left.\varphi_{q}(x) \underset{|x| \rightarrow \infty}{\sim}|x|\right)$. Hence, letting $a, c \rightarrow 0$ and applying the monotone class Theorem, the equality between (1.1.100) and (1.1.101) implies, for every $\Gamma \in b_{+}\left(\mathcal{F}_{\infty}\right)$ :

$$
\mathbf{W}^{-}\left(\Gamma e^{-\frac{1}{2} A_{\infty}^{(q)}}\right)=\left(\int_{0}^{\infty} \psi(y) d y\right) W_{\infty}^{\psi(S)}\left(\frac{\Gamma}{\psi\left(S_{\infty}\right)} e^{-\frac{1}{2} A_{\infty}^{(q)}}\right)
$$

then, replacing $\Gamma e^{-\frac{1}{2} A_{\infty}^{(q)}}$ by $\Gamma$ :

$$
\begin{aligned}
\mathbf{W}^{-}(\Gamma) & =\left(\int_{0}^{\infty} \psi(y) d y\right) \cdot W_{\infty}^{\psi(S)}\left(\frac{\Gamma}{\psi\left(S_{\infty}\right)}\right) \\
& =\mathbf{W}^{-}\left(\psi\left(S_{\infty}\right)\right) W_{\infty}^{\psi(S)}\left(\frac{\Gamma}{\psi\left(S_{\infty}\right)}\right) \\
& =\mathbf{W}\left(\psi\left(S_{\infty}\right)\right) W_{\infty}^{\psi(S)}\left(\frac{\Gamma}{\psi\left(S_{\infty}\right)}\right)
\end{aligned}
$$

since $\psi(\infty)=0$ and $S_{\infty}=+\infty \quad \mathbf{W}^{+}$a.s.

We note that there is no problem to divide by $\psi\left(S_{\infty}\right)$ since $\psi\left(S_{\infty}\right)>0 \quad W_{\infty}^{\psi(S)}$ a.s. (under $W_{\infty}^{\psi(S)}, S_{\infty}$ admits $\psi$ as density (see [RVY, II])).

We have proven (1.1.93), and the proof of (1.1.94) is similar.

ii) We now prove (1.1.95).

For this purpose, we use the penalisation by $\left(e^{-\frac{\lambda}{2} S_{t}}, t \geq 0\right)$ i.e. (1.1.91) and (1.1.92). We obtain :

$$
M_{t}^{\psi(S)}=\left(1+\frac{\lambda}{2}\left(S_{t}-X_{t}\right)\right) e^{-\frac{\lambda}{2} S_{t}}
$$

Hence, for every $t \geq 0$ and $\Gamma_{t} \in b_{+}\left(\mathcal{F}_{t}\right)$ :

$$
\begin{aligned}
W\left(\Gamma_{t}\left(\frac{2}{\lambda}+\left(S_{t}-X_{t}\right)\right)\right. & =\frac{2}{\lambda} W_{\infty}^{\psi(S)}\left[e^{\frac{\lambda S_{t}}{2}} \Gamma_{t}\right] \\
& =\mathbf{W}^{-}\left(e^{-\frac{\lambda}{2}\left(S_{\infty}-S_{t}\right)} \Gamma_{t}\right) \quad(\text { from }(1.1 .93)) \\
& =\mathbf{W}^{-}\left(\Gamma_{t} 1_{\theta_{+} \leq t}\right)+\mathbf{W}^{-}\left(\Gamma_{t} e^{-\frac{\lambda}{2}\left(S_{\infty}-S_{t}\right)} 1_{\theta_{+}>t}\right)
\end{aligned}
$$


We then let $\lambda \rightarrow+\infty$ in (1.1.103) by noting that $S_{\infty}-S_{t}>0$ on $\left(\theta_{+}>t\right)$. We obtain :

$$
W\left(\Gamma_{t}\left(S_{t}-X_{t}\right)\right)=\mathbf{W}^{-}\left(\Gamma_{t} 1_{\theta_{+} \leq t}\right)
$$

This is (1.1.95). By symmetry (1.1.96) now follows.

Remark 1.1.12 We deduce from(1.1.103) and (1.1.95) that:

$$
W\left(\Gamma_{t}\right)\left(\int_{0}^{\infty} e^{-\frac{\lambda}{2} y} d y\right)=\mathbf{W}^{-}\left(\Gamma_{t} e^{-\frac{\lambda}{2}\left(S_{\infty}-S_{t}\right)} 1_{\theta_{+}>t}\right)
$$

and operating as in the proof of point 3), i) of Theorem 1.1.6, we obtain :

$$
\begin{aligned}
\mathbf{W}^{-}\left(S_{\infty}-S_{t} \in d l\right) & =1_{[0, \infty[}(l) d l+W\left(S_{t}-X_{t}\right) \delta_{0}(d l) \\
& =1_{[0, \infty[}[l) d l+\sqrt{\frac{2 t}{\pi}} \delta_{0}(d l)
\end{aligned}
$$

and, conditionally on $S_{\infty}-S_{t}=l, l>0,\left(X_{u}, u \leq t\right)$ is a Brownian motion indexed by $[0, t]$. Theorem 1.1.11 is the prototype of similar results which we may obtain for other penalisations. Here are, without proof, some examples.

Theorem 1.1.11'.

1) Let $h^{+}, h^{-}: \mathbb{R}_{+} \longrightarrow \mathbb{R}_{+}$such that $\int_{0}^{\infty}\left(h^{+}+h^{-}\right)(y) d y<\infty$. Let $W_{\infty}^{h^{+}, h^{-}}$denote the probability defined by (see $[R V Y, I I])$ :

$$
W_{\infty}^{h^{+}, h^{-}}{ }_{\mid \mathcal{F}_{t}}=M_{t}^{h^{+}, h^{-}} \cdot W_{\mid \mathcal{F}_{t}}
$$

with

$$
\begin{gathered}
M_{t}^{h^{+}, h^{-}}=\frac{1}{\frac{1}{2} \int_{0}^{\infty}\left(h^{+}+h^{-}\right)(y) d y}\left\{\left(X_{t}^{+} h^{+}\left(L_{t}\right)+X_{t}^{-} h^{-}\left(L_{t}\right)\right.\right. \\
\left.\left.+\int_{L_{t}}^{\infty} \frac{1}{2}\left(h^{+}+h^{-}\right)(y) d y\right)\right\}
\end{gathered}
$$

Then :

$$
\mathbf{W}=\left\{\mathbf{W}^{+}\left(\left(h^{+}\left(L_{\infty}\right)\right)+\mathbf{W}^{-}\left(h^{-}\left(L_{\infty}\right)\right)\right\}\left(1_{\Gamma_{+}} \frac{1}{h^{+}\left(L_{\infty}\right)}+1_{\Gamma_{-}} \frac{1}{h^{-}\left(L_{\infty}\right)}\right) W_{\infty}^{h^{+}, h^{-}}\right.
$$

In other words :

$$
\begin{aligned}
& \mathbf{W}^{+}=\left\{\mathbf{W}^{+}\left(\left(h^{+}\left(L_{\infty}\right)\right)+\mathbf{W}^{-}\left(h^{-}\left(L_{\infty}\right)\right)\right\} \frac{1_{\Gamma_{+}}}{h^{+}\left(L_{\infty}\right)} W_{\infty}^{h^{+}, h^{-}}\right. \\
& \mathbf{W}^{-}=\left\{\left(\mathbf{W}^{+}\left(h^{+}\left(L_{\infty}\right)\right)+\mathbf{W}^{-}\left(h^{-}\left(L_{\infty}\right)\right)\right\} \frac{1_{\Gamma_{-}}}{h^{-}\left(L_{\infty}\right)} W_{\infty}^{h^{+}, h^{-}}\right.
\end{aligned}
$$

2) Let $\psi: \mathbb{R}_{+} \longrightarrow \mathbb{R}_{+}$be Borel and integrable, and let :

$$
M_{t}^{\psi\left(S_{g}\right)}:=\left(\frac{1}{2} \psi\left(S_{g^{(t)}}\right)\left|X_{t}\right|+\psi\left(S_{t}\right)\left(S_{t}-X_{t}^{+}\right)+\int_{S_{t}}^{\infty} \psi(y) d y\right) \cdot \frac{1}{\int_{0}^{\infty} \psi(y) d y}
$$


with $g^{(t)}:=\sup \left\{s \leq t, X_{s}=0\right\}$ and let $W_{\infty}^{\psi\left(S_{g}\right)}$ defined by :

$$
W_{\infty}^{\psi\left(S_{g}\right)}{ }_{\mid \mathcal{F}_{t}}=M_{t}^{\psi\left(S_{g}\right)} \cdot W_{\mid \mathcal{F}_{t}}
$$

(see $[R Y, V I I I])$. Then :

i) $\quad \mathbf{W}=\mathbf{W}\left(\psi\left(S_{g}\right)\right) \frac{1}{\psi\left(S_{g}\right)} \cdot W_{\infty}^{\psi\left(S_{g}\right)}$

ii) Let $\rho:=\sup \left\{u \leq g, S_{g^{(u)}}<S_{g}\right\}$. Then, for every $t$ and every $\Gamma_{t} \in b_{+}\left(\mathcal{F}_{t}\right)$ :

$$
\mathbf{W}\left(\Gamma_{t} 1_{\rho \leq t}\right)=W\left(\Gamma_{t}\left(\frac{1}{2}\left|X_{t}\right|+\left(S_{t}-X_{t}^{+}\right) 1_{S_{t}=S_{g}^{(t)}}\right)\right)
$$

We could also present analogous results for penalisations associated to the numbers of downcrossings (see [RVY, II]) or the length of the longest excursion before $g^{(t)}$ (see [RVY, VII]), etc...

We use, in Section 2, Theorem 1.1.11 and 1.1.11' to give explicit examples of martingales $\left(M_{t}(F), t \geq 0\right), F \in L_{+}^{1}(\mathbf{W})$. These martingales are defined in Theorem 1.2.1

\subsection{Brownian martingales associated to $W$.}

The notation in this Section 1.2 is the same as in Section 1.1. Our aim here is to associate to every r.v. in $L_{+}^{1}\left(\Omega, \mathcal{F}_{\infty}, \mathbf{W}\right)$ a martingale and to study a few of its properties. Thus, $\mathbf{W}$ appears as "a machine to construct $W$-martingales". We shall also prove (see Theorem 1.2.4) a decomposition Theorem which is valid for every positive Brownian martingale.

1.2.1 Definition of the martingales $\left(M_{t}(F), t \geq 0\right)$.

Theorem 1.2.1. Let $F \in L_{+}^{1}\left(\Omega, \mathcal{F}_{\infty}, \mathbf{W}\right)$. There exists a $\left(\left(\mathcal{F}_{t}, t \geq 0\right), W\right)$ positive (necessarily continuous) martingale $\left(M_{t}(F), t \geq 0\right)$ such that :

1) For every $t \geq 0$ and $\Gamma_{t} \in b(\mathcal{F})$ :

$$
\mathbf{W}\left(F \cdot \Gamma_{t}\right)=W\left(M_{t}(F) \cdot \Gamma_{t}\right)
$$

In particular, for every $t \geq 0$ :

$$
\mathbf{W}(F)=W\left(M_{t}(F)\right)
$$

2) $\quad M_{t}(F)=\widehat{\mathbf{W}}_{X_{t}}\left(F\left(\omega_{t}, \widehat{\omega}^{t}\right)\right)$

(cf Point 1 of Remark 1.2.2 for this notation)

3) $\quad M_{t}(F) \underset{t \rightarrow \infty}{\longrightarrow} 0 \quad W$ a.s.

In particular, the martingale $\left(M_{t}(F), t \geq 0\right)$ is not uniformly integrable.

4) For every $q \in \mathcal{I}$ :

$$
M_{t}(F)=\varphi_{q}(0) M_{t}^{(q)} W_{\infty}^{(q)}\left(F e^{\frac{1}{2} A_{\infty}^{(q)}} \mid \mathcal{F}_{t}\right)
$$

where $M_{t}^{(q)}, \varphi_{q}$ and $W_{\infty}^{(q)}$ are defined in Theorem 1.1.1.

\section{Remark 1.2.2.}

1. We now give some explanation about the notation in (1.2.3). If $\omega \in \mathcal{C}\left(\mathbb{R}_{+} \rightarrow \mathbb{R}\right)$, then $\omega_{t}$ $\left(\right.$ resp. $\left.\omega^{t}\right)$ denotes the part of $\omega$ before $t$ (resp. after $t$ ) :

$$
\omega=\left(\omega_{t}, \omega^{t}\right)
$$


that is, precisely :

$$
X_{u}(\omega)=\left\{\begin{array}{lll}
X_{u}\left(\omega_{t}\right) & \text { if } \quad u \leq t \\
X_{u-t}\left(\omega^{t}\right) & \text { if } \quad u \geq t
\end{array}\right.
$$

and our notation $\widehat{\mathbf{W}}_{X_{t}\left(\omega_{t}\right)}\left(F\left(\omega_{t}, \widehat{\omega}^{t}\right)\right)$ stands for the expectation of $F\left(\omega_{t}, \bullet\right)$ with respect to $\mathbf{W}_{X_{t}(\omega)}$.

2. To every r.v. $G$ in $L_{+}^{1}\left(\Omega, \mathcal{F}_{\infty}, W\right)$ we may of course associate the positive martingale $\left(\left(\widetilde{M}_{t}(G):=W\left(G \mid \mathcal{F}_{t}\right), t \geq 0\right)\right.$. But, contrarily to the description for $M_{t}(F)$ given in Theorem 1.2.1, this is a uniformly integrable martingale.

3. Formula (1.2.5) may seem ambiguous, since the r.v. $W_{\infty}^{(q)}\left(F e^{\frac{1}{2} A_{\infty}^{(q)}} \mid \mathcal{F}_{t}\right)$ is only defined $W_{\infty}^{(q)}$ a.s. But since from (1.1.7), the probability $W_{\infty}^{(q)}$ is absolutely continuous on $\mathcal{F}_{t}$ with respect to $W$, there is in fact no ambiguity. On the other hand, from (1.1.16) :

$$
\mathbf{W}(F)=\varphi_{q}(0) W_{\infty}^{(q)}\left(F \exp \left(\frac{1}{2} A_{\infty}^{(q)}\right)\right)<\infty
$$

as soon as $F \in L^{1}(\mathbf{W})$. Thus, the $\left(\left(\mathcal{F}_{t}, t \geq 0\right), W_{\infty}^{(q)}\right)$ martingale $\left(W_{\infty}^{(q)}\left(F \exp \left(\frac{1}{2} A_{\infty}^{(q)}\right) \mid \mathcal{F}_{t}\right)\right.$, $t \geq 0$ is $W_{\infty}^{(q)}$ uniformly integrable.

4. Of course, $\left(M_{t}(F), t \geq 0\right)$ is continuous. As it is a $\left(\left(\mathcal{F}_{t}, t \geq 0\right), W\right)$ martingale.

5. On the injectivity of $F \longrightarrow\left(M_{t}(F), t \geq 0\right)$. Assume that, for $F_{1}$ and $F_{2}$ belonging to $L^{1}\left(\Omega, \mathcal{F}_{\infty}, \mathbf{W}\right)$ we have : $M_{t}\left(F_{1}\right)=M_{t}\left(F_{2}\right)$ a.s., for every $t \geq 0$. Then $F_{1}=F_{2} \quad \mathbf{W}$ a.s. Indeed, from (1.2.1) :

$$
W\left(\Gamma_{t}\left(M_{t}\left(F_{1}\right)-M_{t}\left(F_{2}\right)\right)=0=W\left(\Gamma_{t} M_{t}\left(F_{1}-F_{2}\right)\right)=\mathbf{W}\left(\Gamma_{t}\left(F_{1}-F_{2}\right)\right)\right.
$$

As this relation is true for every $t \geq 0$ and $\Gamma_{t} \in b\left(\mathcal{F}_{t}\right)$, the monotone class Theorem implies that, for every $\Gamma \in b\left(\mathcal{F}_{\infty}\right)$ :

$$
\mathbf{W}\left(\Gamma\left(F_{1}-F_{2}\right)\right)=0 \text {, i.e. } F_{1}=F_{2} \quad \mathbf{W} \text { a.s. }
$$

Later in this Section we shall obtain a more direct "construction" of $F$ from $\left(M_{t}(F), t \geq 0\right)$. Proof of Theorem 1.2.1.

i) We show point 1 .

We denote by $W^{F}$ the finite positive measure on $\left(\Omega, \mathcal{F}_{\infty}\right)$ defined :

$$
W^{F}(G)=\mathbf{W}(F \cdot G)
$$

Let $\Gamma_{t} \in b_{+}\left(\mathcal{F}_{t}\right)$ such that $W\left(\Gamma_{t}\right)=0$. From (1.1.7), for every $q \in \mathcal{I}, W_{\infty}^{(q)}\left(\Gamma_{t}\right)=0$ hence, from (1.1.16) :

$$
W^{F}\left(\Gamma_{t}\right)=\mathbf{W}\left(F \cdot \Gamma_{t}\right)=\varphi_{q}(0) W_{\infty}^{(q)}\left(F e^{\frac{1}{2} A_{\infty}^{(q)}} \Gamma_{t}\right)=0
$$

from (1.2.6). Thus :

$$
W_{\mid \mathcal{F}_{t}}^{F} \ll W_{\mid \mathcal{F}_{t}}
$$

Consequently, from the Radon-Nikodym Theorem, there exists a $W$ integrable, positive r.v. $M_{t}(F)$, such that

$$
W_{\mid \mathcal{F}_{t}}^{F}=M_{t}(F) \cdot W_{\mid \mathcal{F}_{t}}
$$


This is a rewriting of formula (1.2.1). Formula (1.2.2) is obtained from (1.2.1) by taking $\Gamma_{t} \equiv 1$. The fact that $\left(M_{t}(F), t \geq 0\right)$ is a $\left(\left(\mathcal{F}_{t}, t \geq 0\right), W\right)$ martingale follows from (1.2.8). We also note that, as every Brownian martingale, the process $\left(M_{t}(F), t \geq 0\right)$ admits a continuous version (which we shall always consider).

ii) We show point 4 .

From (1.2.1), (1.1.16) and (1.1.7), we have for every $\Gamma_{t} \in b_{+}\left(\mathcal{F}_{t}\right)$

$$
\begin{aligned}
\mathbf{W}\left(\Gamma_{t} F\right) & =W\left(\Gamma_{t} M_{t}(F)\right) \\
& =\varphi_{q}(0) W_{\infty}^{(q)}\left(\Gamma_{t} F e^{\frac{1}{2} A_{\infty}^{(q)}}\right) \quad(\text { from }(1.1 .16)) \\
& =\varphi_{q}(0) W_{\infty}^{(q)}\left(\Gamma_{t} W_{\infty}^{(q)}\left(F e^{\frac{1}{2} A_{\infty}^{(q)}} \mid \mathcal{F}_{t}\right)\right) \quad(\text { from }(1.1 .7)) \\
& =\varphi_{q}(0) W\left(\Gamma _ { t } M _ { t } ^ { ( q ) } W _ { \infty } ^ { ( q ) } \left(F e^{\left.\left.\frac{1}{2} A_{\infty}^{(q)} \mid \mathcal{F}_{t}\right)\right)}\right.\right.
\end{aligned}
$$

(1.2.5) follows.

iii) We show point 3 .

- For every $s \geq 0$ and $\Gamma_{s} \in b\left(\mathcal{F}_{s}\right)$, we have for $s \leq t$ from $(1.2 .1)$ :

$$
\mathbf{W}\left(\Gamma_{s} \cdot F\right)=W\left(\Gamma_{s} \cdot M_{t}(F)\right)
$$

Since the $\left(\left(\mathcal{F}_{t}, t>0\right), W\right)$ martingale $\left(M_{t}(F), t \geq 0\right)$ is positive, it converges $W$ a.s. towards $M_{\infty}(F)$. Letting $t \rightarrow \infty$ in (1.2.9) and using Fatou's Lemma, we have :

$$
W\left(\Gamma_{s} M_{\infty}(F)\right) \leq \mathbf{W}\left(\Gamma_{s} \cdot F\right)
$$

Choosing $\Gamma_{s}=\left\{1_{g^{(s)} \geq a}\right\}$, with $g^{(s)}:=\sup \left\{u \leq s, X_{u}=0\right\}$ we obtain :

$$
W\left(1_{g^{(s)} \geq a} \cdot M_{\infty}(F)\right) \leq \mathbf{W}\left(1_{g^{(s)} \geq a} \cdot F\right)
$$

Letting $s \rightarrow \infty$ in (1.2.10) and noting that :

$$
\begin{array}{ll}
1_{g^{(s)} \geq a} \longrightarrow 1 & W \text { a.s. } \\
1_{g^{(s)} \geq a} \longrightarrow 1_{g \geq a} & \mathbf{W} \text { a.s.. }
\end{array}
$$

we obtain :

$$
W\left(M_{\infty}(F)\right) \leq \mathbf{W}\left(1_{g \geq a} \cdot F\right)
$$

Now, from Theorem 1.1.6 we know that $g<\infty \mathbf{W}$ a.s., hence we get : $\mathbf{W}\left(1_{g \geq a} \cdot F\right) \underset{a \rightarrow \infty}{\longrightarrow} 0$. Thus :

$$
W\left(M_{\infty}(F)\right)=0 \quad \text { and } \quad M_{\infty}(F)=0 \quad W \text { a.s. }
$$

- Another way to prove point 3. consists in writing, for $s \leq t$ :

$$
\begin{aligned}
W\left(\Gamma_{s} M_{t}(F)\right) & =\varphi_{q}(0) W\left(\Gamma_{s} M_{t}^{(q)} W_{\infty}^{(q)}\left(F e^{\frac{1}{2} A_{\infty}^{(q)}} \mid \mathcal{F}_{t}\right)\right) \quad(\text { from }(1.2 .5)) \\
& =\varphi_{q}(0) W_{\infty}^{(q)}\left(\Gamma_{s} W_{\infty}^{(q)}\left(F e^{\frac{1}{2} A_{\infty}^{(q)}} \mid \mathcal{F}_{t}\right)\right) \quad(\text { from }(1.1 .7))
\end{aligned}
$$

But, since the $W_{\infty}^{(q)}$ martingale $\left(W_{\infty}^{(q)}\left(F e^{\frac{1}{2} A_{\infty}^{(q)}} \mid \mathcal{F}_{t}\right), t \geq 0\right)$ is uniformly integrable it converges a.s. and in $L^{1}\left(W_{\infty}^{(q)}\right)$ towards $F e^{\frac{1}{2} A_{\infty}^{(q)}}$ as $t \rightarrow \infty$. Thus, letting $t \rightarrow \infty$ in (1.2.11) and using again Fatou's Lemma, we obtain :

$$
W\left(\Gamma_{s} M_{\infty}(F)\right) \leq \varphi_{q}(0) W_{\infty}^{(q)}\left(\Gamma_{s} F e^{\frac{1}{2} A_{\infty}^{(q)}}\right)
$$


We then choose $\Gamma_{s}=\mathbf{1}_{\left\{A_{s}^{(a)} \geq a\right\}}$ and obtain

$$
W\left(1_{\left(A_{s}^{(q)} \geq a\right)} M_{\infty}(F)\right) \leq \varphi_{q}(0) W_{\infty}^{(q)}\left(1_{A_{s}^{(q)} \geq a} F e^{-\frac{1}{2} A_{\infty}^{(q)}}\right)
$$

We then let $s \rightarrow \infty$ and note that :

$$
\begin{aligned}
& 1_{A_{s}^{(q)} \geq a} \longrightarrow 1 \quad W \text { a.s. (since Brownian motion is recurrent) } \\
& 1_{A_{s}^{(q)} \geq a} \longrightarrow 1_{A_{\infty}^{(q)} \geq a} W_{\infty}^{(q)} \text { a.s. }
\end{aligned}
$$

Hence :

$$
W\left(M_{\infty}(F)\right) \leq \varphi_{q}(0) W_{\infty}^{(q)}\left(1_{A_{s}^{(q)} \geq a} F e^{-\frac{1}{2} A_{\infty}^{(q)}}\right)
$$

It now suffices to let $a \rightarrow \infty$, using the fact that $A_{\infty}^{(q)}<\infty W_{\infty}^{(q)}$ a.s. (this is given by Lemma 3.1 ) and that $F e^{-\frac{1}{2} A_{\infty}^{(q)}} \in L^{1}\left(W_{\infty}^{(q)}(\right.$ from $(1.2 .6))$ to obtain :

$$
W\left(M_{\infty}(F)\right)=0 \quad \text { and hence : } M_{\infty}(F)=0 \quad W \text { a.s. }
$$

iv) We prove point 2.

We have, from (1.2.5) :

$$
\begin{aligned}
M_{t}(F)= & \varphi_{q}(0) M_{t}^{(q)} W_{\infty}^{(q)}\left(F e^{-\frac{1}{2} A_{\infty}^{(q)}} \mid \mathcal{F}_{t}\right) \\
= & \varphi_{q}\left(X_{t}\right) e^{-\frac{1}{2} A_{t}^{(q)}} W_{\infty}^{(q)}\left(F e^{-\frac{1}{2} A_{\infty}^{(q)}} \mid \mathcal{F}_{t}\right) \\
& \left(\text { from the definition }(1.1 .8) \text { of } M_{t}^{(q)}\right) \\
= & \varphi_{q}\left(X_{t}\right) \widehat{W}_{X_{t}, \infty}^{(q)}\left(e^{\frac{1}{2}\left(A_{\infty}^{q}-A_{t}^{q}\right)} F\left(\omega_{t}, \widehat{\omega}^{t}\right)\right) \\
& (\text { from the Markov property }) \\
= & \widehat{\mathbf{W}}_{X_{t}}\left(F\left(\omega_{t}, \widehat{\omega}^{t}\right), \quad \text { from }(1.1 .16)\right.
\end{aligned}
$$

1.1.2.2 Examples of martingales $\left(M_{t}(F), t \geq 0\right)$.

Formula (1.2.3) which provides an "explicit" expression for $M_{t}(F)$ is not always, practically, easy to compute.

2.2.1 A first method to obtain examples of $\left(M_{t}(F), t \geq 0\right)$.

To begin with, we present a "computation principle" to obtain $M_{t}(F)$.

"Computation principle"

$\overline{\text { Let }\left(N_{t}, t \geq 0\right) \text { denote a }}\left(\left(\mathcal{F}_{t}, t \geq 0\right), W\right)$ positive martingale such that $N_{0}=1$. Let $W_{\infty}^{N}$ be the probability on $\left(\Omega, \mathcal{F}_{\infty}\right)$ which is characterized by :

$$
W_{\infty \mid \mathcal{F}_{t}}^{N}=N_{t} \cdot W_{\mid \mathcal{F}_{t}}
$$

Let us assume that there exists a r.v. $F \in L_{+}^{1}\left(\Omega, \mathcal{F}_{\infty}, \mathbf{W}\right)$ such that :

$$
F \cdot \mathbf{W}=\mathbf{W}(F) \cdot W_{\infty}^{N}
$$

Then

$$
M_{t}(F)=\mathbf{W}(F) \cdot N_{t}
$$




\section{Proof of the "Computation principle".}

We have, for every $t \geq 0$ and $\Gamma_{t} \in b\left(\mathcal{F}_{t}\right)$, from (1.2.1) :

$$
\mathbf{W}\left(F \cdot \Gamma_{t}\right)=W\left(M_{t}(F) \cdot \Gamma_{t}\right)
$$

On the other hand, from the hypothesis (1.2.16) :

$$
\mathbf{W}\left(F \cdot \Gamma_{t}\right)=\mathbf{W}(F) W_{\infty}^{N}\left(\Gamma_{t}\right)
$$

Hence, this quantity also equals :

$$
\mathbf{W}(F) W\left(\Gamma_{t} \cdot N_{t}\right)
$$

from (1.2.15). Since $\Gamma_{t}$ denotes any $\mathcal{F}_{t}$ measurable set in (1.2.18), one obtains :

$$
M_{t}(F)=\mathbf{W}(F) \cdot N_{t} \quad W \text { a.s. }
$$

Example 1. Let $q \in \mathcal{I}$ and $N_{t}:=\frac{\varphi_{q}\left(X_{t}\right)}{\varphi_{q}(0)} \exp \left(-\frac{1}{2} A_{t}^{(q)}\right)$.

From (1.1.16) and (1.1.7), the hypotheses of the "Computation principle" are satisfied with $F=\exp \left(-\frac{1}{2} A_{t}^{(q)}\right)$. Thus :

$$
\begin{aligned}
M_{t}\left(e^{-\frac{1}{2} A_{\infty}^{q}}\right) & =\mathbf{W}\left(e^{-\frac{1}{2} A_{\infty}^{q}}\right) \cdot \frac{\varphi_{q}\left(X_{t}\right)}{\varphi_{q}(0)} \exp \left(-\frac{1}{2} A_{t}^{(q)}\right) \\
& =\varphi_{q}\left(X_{t}\right) \exp \left(-\frac{1}{2} A_{t}^{(q)}\right)
\end{aligned}
$$

since, from (1.1.17), $\mathbf{W}\left(\exp \left(\frac{1}{2} A_{t}^{(q)}\right)\right)=\varphi_{q}(0)$.

Example 2. Let $h: \mathbb{R}_{+} \rightarrow \mathbb{R}_{+}$Borel and integrable and :

$$
N_{t}:=\frac{1}{\int_{0}^{\infty} h(y) d y} \cdot\left(h\left(L_{t}\right)\left|X_{t}\right|+\int_{L_{t}}^{\infty} h(y) d y\right)
$$

From Theorem 1.1.11', the hypotheses of the "Computation principle" are satisfied with $F=h\left(L_{\infty}\right)$ (we note from point 3, iii) of Theorem 1.1.6: $\left.\mathbf{W}\left(h\left(L_{\infty}\right)\right)=\int_{0}^{\infty} h(l) d l<\infty\right)$. Thus :

$$
M_{t}\left(h\left(L_{\infty}\right)\right)=h\left(L_{t}\right)\left|X_{t}\right|+\int_{L_{t}}^{\infty} h(y) d y
$$

(cf [RVY, II] for the use of this martingale).

Example 3. Let $S_{t}:=\sup _{s \leq t} X_{s}$ and $\psi: \mathbb{R}_{+} \rightarrow \mathbb{R}_{+}$Borel and integrable, such that $\psi(+\infty)=0$. Due to Theorem 1.1.11, the "Computation principle" applies with $F=\psi\left(S_{\infty}\right)$ and

$$
N_{t}:=\frac{1}{\int_{0}^{\infty} \psi(y) d y}\left(\psi\left(S_{t}\right)\left(S_{t}-X_{t}\right)+\int_{S_{t}}^{\infty} \psi(y) d y\right)
$$

We note that, from (1.1.104) (taken with $t=0)$ :

$$
\mathbf{W}\left(\psi\left(S_{\infty}\right)\right)=\int_{0}^{\infty} \psi(l) d l<\infty .
$$


Thus :

$$
M_{t}\left(\psi\left(S_{\infty}\right)\right)=\psi\left(S_{t}\right)\left(S_{t}-X_{t}\right)+\int_{S_{t}}^{\infty} \psi(y) d y
$$

Another manner to obtain (1.2.23) make be to invoke Lévy's Theorem :

$$
\left(\left(S_{t}, S_{t}-X_{t}\right), t \geq 0\right) \stackrel{(\text { law })}{=}\left(\left(L_{t},\left|X_{t}\right|\right), t \geq 0\right)
$$

then to use (1.2.21).

The reader may refer to [RVY, II] for links between the Azéma-Yor martingale $\left(\psi\left(S_{t}\right)\left(S_{t}-X_{t}\right)\right.$ $\left.+\int_{S_{t}}^{\infty} \psi(y) d y, t \geq 0\right)$ and the penalisation problem with the process $\left(\psi\left(S_{t}\right), t \geq 0\right)$.

Example 4. Let $\psi: \mathbb{R}_{+} \rightarrow \mathbb{R}_{+}$Borel, integrable function with $\psi(\infty)=0$. The "Computation principle" thanks to Theorem 1.1.11', yields to, with $F=\psi\left(S_{g}\right)$ :

$$
\begin{aligned}
M_{t}\left(\psi\left(S_{g}\right)\right) & =\frac{1}{2} \psi\left(S_{g^{(t)}}\right)\left|X_{t}\right|+\psi\left(S_{t}\right)\left(S_{t}-X_{t}^{+}\right)+\int_{S_{t}}^{\infty} \psi(y) d y \\
& =\frac{1}{2} \psi\left(S_{g^{(t)}}\right) \cdot X_{t}+M_{t}\left(\psi\left(S_{\infty}\right)\right)
\end{aligned}
$$

where $M_{t}\left(\psi\left(S_{\infty}\right)\right.$ is defined by (1.2.23). We note that, from (1.1.104), since $\psi(+\infty)=0$ :

$$
\mathbf{W}^{-}\left(\psi\left(S_{g}\right)\right)=\mathbf{W}\left(\psi\left(S_{\infty}\right)\right)=\int_{0}^{\infty} \psi(l) d l<\infty
$$

Example 5. Let $a<b$ and :

$$
\begin{array}{llll}
T^{(1)} & :=\inf \left\{t \geq 0 ; X_{t}>b\right\}, & T^{(2)} & :=\inf \left\{t \geq T^{(1)} ; X_{t}<a\right\} \\
T^{(2 n+1)} & :=\inf \left\{t \geq T^{(2 n)} ; X_{t}>b\right\} & T^{(2 n+2)} & :=\inf \left\{t \geq T^{(2 n+1)} ; X_{t}<a\right\}
\end{array}
$$

Define :

$$
D_{t}^{[a, b]}:=\sum_{n \geq 1} 1_{\left(T^{(2 n)} \leq t\right)}
$$

$D_{t}^{[a, b]}$ is the number of down crossings on the interval $[a, b]$ before time $t$. Soit $h: \mathbb{N} \rightarrow \mathbb{R}_{+}$ such that $h$ is decreasing, $h(0)=1, h(+\infty)=0$ and denote $\Delta h(n):=h(n)-h(n+1)$. The "Computation principle" and an extension to this situation of Theorem 1.1.11' lead to :

$$
\begin{aligned}
M_{t}\left(\Delta h\left(D_{\infty}^{[a, b]}\right)\right)= & \sum_{n \geq 0}\left\{1_{\left[T^{(2 n)}, T^{(2 n+1)}[\right.}(t)\left[\frac{h(n)}{2}\left(1+\frac{b-X_{t}}{b-a}\right)+\frac{h(n+1)}{2}\left(\frac{X_{t}-a}{b-a}\right)\right]\right. \\
& \left.+1_{\left[T^{(2 n+1)}, T^{(2 n+2)}[\right.}(t)\left[\frac{h(n+1)}{2}\left(1+\frac{b-X_{t}}{b-a}\right)+\frac{h(n)}{2}\left(\frac{X_{t}-a}{b-a}\right)\right]\right\}
\end{aligned}
$$

The reader may refer to [RVY, II] for more information relative to this martingale.

Example 6. Let $\Sigma_{g^{(t)}}$ denote the length of the longest excursion of Brownian motion $\left(X_{u}, u \geq 0\right)$ before $g^{(t)}:=\sup \left\{s \leq t ; X_{s}=0\right\}$. Let $h: \mathbb{R}_{+} \rightarrow \mathbb{R}_{+}$such that 
$\int_{0}^{\infty} z\left|h^{\prime}(z)\right| d z<\infty$. Then, the "Computation principle" and an extension of Theorem 1.1.11', lead to :

$$
\begin{aligned}
M_{t}\left(\sqrt{h}\left(\Sigma_{g}\right)\right)= & \sqrt{h}\left(\Sigma_{g^{(t)}}\right) \cdot\left|X_{t}\right|+h_{1}\left(A_{t}\right) \Phi\left(\frac{\left|X_{t}\right|}{\sqrt{\left(\Sigma_{g^{(t)}}-A_{t}\right)_{+}}}\right) \\
& +\int_{0}^{\frac{\left|X_{t}\right|}{\sqrt{\left(\Sigma_{g^{(t)}}-A_{t}\right)_{+}}}} h_{1}\left(A_{t}+\frac{X_{t}^{2}}{v^{2}}\right)\left(\exp \left(-\frac{v^{2}}{2}\right)\right) d v
\end{aligned}
$$

with

$$
\begin{aligned}
& A_{t}:=t-g^{(t)}, \Phi(x):=\int_{x}^{\infty} \exp \left(-\frac{v^{2}}{2}\right) d v \\
& h_{1}(x):=-\int_{\sqrt{x}}^{\infty} z h^{\prime}(z) d z
\end{aligned}
$$

(see [RY, VIII] or [RY, M], Chap. 3).

1.2.2.2 A second manner to compute explicitely martingales of the form $\left(M_{t}(F), t \geq 0\right)$.

This method hinges upon the following Theorem 1.2.3. $\left(F_{u}, u \geq 0\right)$ denotes a positive predictable process such that :

$$
\mathbf{W}\left(F_{g}\right)<\infty
$$

We note that from Theorem 1.1.6, this condition is equivalent to :

$$
W\left(\int_{0}^{\infty} F_{s} d L_{s}\right)<\infty
$$

or equivalently after the change of variable $l=L_{s}$, to :

$$
\int_{0}^{\infty} W\left(F_{\tau_{l}}\right) d l=W\left(\int_{0}^{\infty} F_{s} d L_{s}\right)<\infty
$$

with :

$$
\tau_{l}:=\inf \left\{t>0 ; L_{t}>l\right\}
$$

Theorem 1.2.3. Let $\left(F_{u}, u \geq 0\right)$ denote a positive predictable process such that:

$$
\mathbf{W}\left(F_{g}\right)=\int_{0}^{\infty} W\left(F_{\tau_{l}}\right) d l<\infty
$$

Then, the martingale $\left(M_{t}\left(F_{g}\right), t \geq 0\right)$ may be expressed as :

$$
\begin{aligned}
M_{t}\left(F_{g}\right) & =F_{g^{(t)}} \cdot\left|X_{t}\right|+\int_{t}^{\infty} p_{u-t}\left(X_{t}\right) \Pi_{0,0}^{(u)}\left(F_{u} \mid \mathcal{F}_{t}\right) d u \\
& =F_{g^{(t)}} \cdot\left|X_{t}\right|+\int_{L_{t}}^{\infty} W\left(F_{\tau_{l}} \mid \mathcal{F}_{t}\right) d l \\
& =\int_{0}^{t} F_{g^{(s)}} \operatorname{sgn}\left(X_{s}\right) d X_{s}+W\left(\int_{0}^{\infty} F_{\tau_{l}} d l \mid \mathcal{F}_{t}\right)
\end{aligned}
$$


In (1.2.33) $\Pi_{0,0}^{(u)}$ denotes the law of Brownian bridge of length $u$ and :

$$
p_{s}(x):=\frac{1}{\sqrt{2 \pi s}} e^{-\frac{x^{2}}{2 s}}
$$

\section{Proof of Theorem 1.2.3.}

i) We first prove (1.2.33).

For every $t \geq 0$ and $\Gamma_{t} \in b\left(\mathcal{F}_{t}\right)$ we have by (1.2.11):

$$
\begin{aligned}
\mathbf{W}\left(\Gamma_{t} F_{g}\right)= & W\left(\Gamma_{t} M_{t}(g)\right) \\
= & \mathbf{W}\left(\Gamma_{t} F_{g} 1_{g \leq t}\right)+\mathbf{W}\left(\Gamma_{t} F_{g} 1_{g>t}\right) \\
= & \mathbf{W}\left(\Gamma_{t} F_{g^{(t)}} 1_{g \leq t}\right)+\mathbf{W}\left(\Gamma_{t} F_{g} 1_{g>t}\right) \\
& \left(\text { since } g=g^{(t)} \text { on the set }(g \leq t)\right) \\
:= & \left(1_{t}\right)+\left(2_{t}\right)
\end{aligned}
$$

We study successively $\left(1_{t}\right)$ and $\left(2_{t}\right)$ :

$$
\begin{aligned}
\left(1_{t}\right)= & \mathbf{W}\left(\Gamma_{t} F_{g^{(t)}} 1_{g \leq t}\right) \\
= & W\left(\Gamma_{t} F_{g^{(t)}}\left|X_{t}\right|\right) \\
\left(2_{t}\right)= & \mathbf{W}\left(\Gamma_{t} F_{g} 1_{g>t}\right) \\
= & \int_{t}^{\infty} \frac{d u}{\sqrt{2 \pi u}} \mathbf{W}\left(\Gamma_{t} F_{g} \mid g=u\right) \\
= & \int_{t}^{\infty} \frac{d u}{\sqrt{2 \pi u}} \mathbf{W}\left(\Gamma_{t} F_{u} \mid g=u\right) \\
= & \int_{t}^{\infty} \frac{d u}{\sqrt{2 \pi u}} \Pi_{0,0}^{(u)}\left(\Gamma_{t} F_{u}\right) \quad(\text { from Theorem 1.1.6) } \\
= & \int_{t}^{\infty} \frac{d u}{\sqrt{2 \pi u}} \Pi_{0,0}^{(u)}\left(\Gamma_{t} \Pi_{0,0}^{(u)}\left(F_{u} \mid \mathcal{F}_{t}\right)\right)
\end{aligned}
$$

We now use the (partial) absolute continuity formula for the law of the Brownian bridge with respect to that of Brownian motion :

$$
\Pi_{0,0 \mid \mathcal{F}_{t}}^{(u)}=\frac{p_{u-t}\left(X_{t}\right)}{p_{u}(0)} \cdot W_{\mid \mathcal{F}_{t}}
$$

to obtain :

$$
\begin{aligned}
\left(2_{t}\right) & =\int_{t}^{\infty} \frac{d u}{\sqrt{2 \pi u}} W\left(\frac{\Gamma_{t} p_{u-t}\left(X_{t}\right)}{p_{u}(0)} \Pi_{0,0}^{(u)}\left(F_{u} \mid \mathcal{F}_{t}\right)\right) \\
& =\int_{t}^{\infty} d u\left(W\left(\Gamma_{t} p_{u-t}\left(X_{t}\right) \Pi_{0,0}^{(u)}\left(F_{u} \mid \mathcal{F}_{t}\right)\right)\right.
\end{aligned}
$$

since $p_{u}(0)=\frac{1}{\sqrt{2 \pi u}}$. Gathering (1.2.40), (1.2.37) and (1.2.38), we obtain (1.2.33).

ii) We now prove (1.2.34). 
Of course, (1.2.34) is equivalent to :

$$
\int_{t}^{\infty} p_{u-t}\left(X_{t}\right) \Pi_{0,0}^{(u)}\left(F_{u} \mid \mathcal{F}_{t}\right) d u=\int_{L_{t}}^{\infty} W\left(F_{\tau_{l}} \mid \mathcal{F}_{t}\right) d l
$$

or to, for any $\Gamma_{t} \in b\left(\mathcal{F}_{t}\right)$ :

$$
W\left(\Gamma_{t} \int_{t}^{\infty} p_{u-t}\left(X_{t}\right) \Pi_{0,0}^{(u)}\left(F_{u} \mid \mathcal{F}_{t}\right) d u\right)=W\left(\Gamma_{t} \cdot \int_{L_{t}}^{\infty} F_{\tau_{l}} d l\right)
$$

But we have :

$$
\begin{aligned}
W\left(\Gamma_{t} \cdot \int_{L_{t}}^{\infty} F_{\tau_{l}} d l\right)= & W\left(\Gamma_{t} \int_{t}^{\infty} F_{u} d L_{u}\right) \\
& \text { after the change of variable } l=L_{u} \\
= & \int_{t}^{\infty} \frac{d u}{\sqrt{2 \pi u}} \Pi_{0,0}^{(u)}\left(F_{u} \Gamma_{t}\right) \quad(\text { from (1.1.62)) } \\
= & \int_{t}^{\infty} \frac{d u}{\sqrt{2 \pi u}} \Pi_{0,0}^{(u)}\left(\Gamma_{t} \Pi_{0,0}^{(u)}\left(F_{u} \mid \mathcal{F}_{t}\right)\right) \\
= & \int_{t}^{\infty} \frac{d u}{\sqrt{2 \pi u}} W\left(\Gamma_{t} \frac{p_{u-t}\left(X_{t}\right)}{p_{u}(0)} \Pi_{0,0}^{(u)}\left(F_{u} \mid \mathcal{F}_{t}\right)\right) \\
& (\text { by the absolute continuity formula }(1.2 .39)) \\
= & W\left(\Gamma_{t} \int_{t}^{\infty} p_{u-t}\left(X_{t}\right) \Pi_{0,0}^{(u)}\left(F_{u} \mid \mathcal{F}_{t}\right) d u\right)
\end{aligned}
$$

$\left.{ }_{i i}{ }^{\prime}\right)$ We give now a direct proof - i.e. without use (1.2.33) - of (1.2.34). We have, for every $t \geq \overline{0 \Gamma_{t} \in b_{+}\left(\mathcal{F}_{t}\right):}$

$$
\begin{aligned}
\mathbf{W}\left(F_{g} \Gamma_{t}\right) & =\mathbf{W}\left(F_{g} \Gamma_{t} 1_{g \leq t}\right)+\mathbf{W}\left(F_{g} \Gamma_{t} 1_{g>t}\right) \\
& =\mathbf{W}\left(F_{g^{(t)}} \Gamma_{t} 1_{g \leq t}\right)+\mathbf{W}\left(\widetilde{\Gamma}_{g} F_{g}\right)
\end{aligned}
$$

since $g=g^{(t)}$ on the set $(g \leq t)$ and using the notation :

$$
\begin{aligned}
& \left(\widetilde{\Gamma}_{u}, u \geq 0\right):=\left(\Gamma_{t} 1_{] t, \infty[}(u), u \geq 0\right) \\
& \quad=W\left(\Gamma_{t} F_{g^{(t)}}\left|X_{t}\right|+W\left(\int_{0}^{\infty} \widetilde{\Gamma}_{\tau_{l}} F_{\tau_{l}} d l\right)\right.
\end{aligned}
$$

(from point 2 i) of Theorem 1.1.6 and from formula (1.1.44)).

Hence :

$$
\begin{aligned}
\mathbf{W}\left(\widetilde{\Gamma}_{g} F_{g}\right) & =W\left(\int_{0}^{\infty} \widetilde{\Gamma}_{u} F_{u} d L_{u}\right) \\
& =W\left(\int_{0}^{\infty} \widetilde{\Gamma}_{\tau_{l}} F_{\tau_{l}} d l\right)
\end{aligned}
$$

Finally :

$$
\begin{aligned}
\mathbf{W}\left(F_{g} \Gamma_{t}\right) & =W\left(\Gamma_{t} F_{g^{(t)}}\left|X_{t}\right|\right)+W\left(\Gamma_{t} \int_{0}^{\infty} 1_{t<\tau_{l}} F_{\tau_{l}} d l\right) \\
& =W\left(\Gamma_{t} F_{g^{(t)}}\left|X_{t}\right|\right)+W\left(\Gamma_{t} \int_{L_{t}}^{\infty} F_{\tau_{l}} d l\right) \\
& =W\left(\Gamma_{t} F_{g^{(t)}}\left|X_{t}\right|\right)+W\left(\Gamma_{t} \int_{L_{t}}^{\infty} W\left(F_{\tau_{l}} \mid \mathcal{F}_{t}\right) d l\right)
\end{aligned}
$$


which implies (1.2.34).

iii) We now prove (1.2.35).

To go from (1.2.34) to (1.2.35), we use the balayage formulae, which yields :

$$
F_{g^{(t)}} \cdot\left|X_{t}\right|=\int_{0}^{t} F_{g^{(s)}} \operatorname{sgn}\left(X_{s}\right) d X_{s}+\int_{0}^{t} F_{u} d L_{u}
$$

and we add this expression to $\int_{L_{t}}^{\infty} W\left(F_{\tau_{l}} \mid \mathcal{F}_{t}\right) d l=W\left(\int_{t}^{\infty} F_{u} d L_{u} \mid \mathcal{F}_{t}\right)$ on the RHS. It is now clear that (1.2.34) implies (1.2.35).

\section{Corollary 1.2.4.}

1) Formula (1.2.34) expresses the martingale $\left(M_{t}\left(F_{g}\right), t \geq 0\right)$ as the sum of a submartingale $\left(F_{g^{(t)}} \cdot\left|X_{t}\right|, t \geq 0\right)$ and a supermartingale $\left(W\left(\int_{0}^{\infty} F_{\tau_{l}} 1_{\tau_{l}>t} d l \mid \mathcal{F}_{t}\right), t \geq 0\right)$ both of which converge to 0 a.s., as $t \rightarrow \infty$.

2) The variable $\int_{0}^{\infty} F_{g^{(u)}}^{2} d u$ is finite a.s. but it satisfies :

$$
W\left(\left(\int_{0}^{\infty} F_{g^{(u)}}^{2} d u\right)^{\frac{1}{2}}\right)=+\infty
$$

unless $F_{g}=0, \mathbf{W}$ a.s.

\section{Proof of Corollary 1.2.4.}

The first statement is obvious since $F_{g^{(t)}}\left|X_{t}\right|$ is the absolute value of the martingale $F_{g^{(t)}} \cdot X_{t}$. Moreover, $\left|F_{g^{(t)}} \cdot X_{t}\right| \leq M_{t}\left(F_{g}\right)$, hence since $M_{t}\left(F_{g}\right) \underset{t \rightarrow \infty}{\longrightarrow} 0$ a.s. (see Theorem 1.2.1) the same is true for $F_{g^{(t)}} \cdot X_{t}$. To prove the second item, assume that :

$$
W\left(\left(\int_{0}^{\infty} F_{g^{(u)}}^{2} d u\right)^{\frac{1}{2}}\right)<\infty
$$

Then, the martingale $\left(\int_{0}^{t} F_{g^{(s)}} \operatorname{sgn}\left(X_{s}\right) d X_{s}, t \geq 0\right)$ would be in $H^{1} ;$ a fortiori it would be uniformly integrable. From (1.2.35), since $W\left(\int_{0}^{\infty} F_{\tau_{l}} d l\right)<\infty,\left(M_{t}\left(F_{g}\right), t \geq 0\right)$ would also be uniformly integrable ; but this is only possible, since this martingale converges a.s. to 0 (see Theorem 1.2.1) if it is identically equal to 0 , that is $F_{g}=0 \quad \mathbf{W}$ a.s. (see point 3 of Remark 1.2.2).

Of course, if we want to compute $\left(M_{t}(F), t \geq 0\right)$ in a completely explicit manner, one needs to compute $\Pi_{0,0}^{(u)}\left(F_{u} \mid \mathcal{F}_{t}\right)$, for $t \leq u\left(\right.$ or $\left.W\left(\int_{0}^{\infty} F_{\tau_{l}} d l \mid \mathcal{F}_{t}\right)\right)$. This is what has been done in the Examples 4 and 6 above. Here is an example where this computation is immediate.

Example 7. Let $\psi: \mathbb{R}_{+} \rightarrow \mathbb{R}_{+}$Borel such that :

$$
\int_{0}^{\infty} \psi(t) \frac{d t}{\sqrt{2 \pi t}}<\infty
$$


Then :

$$
M_{t}(\psi(g))=\psi\left(g^{(t)}\right)\left|X_{t}\right|+\int_{0}^{\infty} \frac{d u}{\sqrt{2 \pi u}} e^{-\frac{X_{t}^{2}}{2 u}} \psi(t+u)
$$

To obtain (1.2.45), we apply Theorem 1.2.3 with the (deterministic) process $\left(F_{u}, u \geq 0\right):=$ $(\psi(u), u \geq 0)$ and we use :

$$
\Pi_{0,0}^{(u)}\left(F_{u} \mid \mathcal{F}_{t}\right)=\Pi_{0,0}^{(u)}\left(\psi(u) \mid \mathcal{F}_{t}\right)=\psi(u)
$$

We then make, in (1.2.33) the change of variable $u-t=v$.

More generally (see Theorem 1.1.8), with $g_{a}:=\sup \left\{t ; X_{t}=a\right\}$, we have :

$$
M_{t}\left[\psi\left(g_{a}\right)\right]=\psi\left(g_{a}^{(t)}\right)\left|X_{t}-a\right|+\int_{0}^{\infty} \frac{d u}{\sqrt{2 \pi u}} e^{-\frac{\left(X_{t}-a\right)^{2}}{2 u}} \psi(t+u)
$$

with :

$$
g_{a}^{(t)}:=\sup \left\{s \leq t ; X_{s}=a\right\}
$$

Back to Example 2. Formula (1.2.21) is a particular case of (1.2.34). Indeed, if we apply (1.2.34) with $\left(F_{u}, u \geq 0\right):=\left(h\left(L_{u}\right), u \geq 0\right)$, we obtain :

$$
\begin{aligned}
M_{t}\left(h\left(L_{\infty}\right)\right) & =M_{t}\left(h\left(L_{g}\right)\right) \\
& =h\left(L_{g^{(t)}}\right)\left|X_{t}\right|+W\left(\int_{L_{t}}^{\infty} h\left(L_{\tau_{l}}\right) d l \mid \mathcal{F}_{t}\right) \\
& =h\left(L_{t}\right)\left|X_{t}\right|+\int_{L_{t}}^{\infty} h(l) d l
\end{aligned}
$$

since $L_{g^{(t)}}=L_{t}$ and $L_{\tau_{l}}=l$.

In the same spirit, for $h: \mathbb{R}_{+} \times \mathbb{R}_{+} \rightarrow \mathbb{R}_{+}$Borel such that :

$$
\int_{0}^{\infty} \int_{0}^{\infty} h(l, u) \frac{l e^{-\frac{l^{2}}{2 u}}}{\sqrt{2 \pi u^{3}}} d l d u<\infty
$$

then $($ see $(1.1 .47)) \mathbf{W}\left(h\left(L_{\infty}, g\right)\right)<\infty$ and

$$
\begin{gathered}
M_{t}\left(h\left(L_{\infty}, g\right)\right)=h\left(L_{g^{(t)}}, g^{(t)}\right) \cdot\left|X_{t}\right|+W\left(\int_{L_{t}}^{\infty} h\left(L_{\tau_{l}}, \tau_{l}\right) d l \mid \mathcal{F}_{t}\right) \\
=h\left(L_{t}, g^{(t)}\right)+\widehat{W}_{X_{t}}\left(\int_{0}^{\infty} h\left(L_{t}+\widehat{L}_{v}, t+v\right) \widehat{d L}_{v}\right)
\end{gathered}
$$

1.2.2.3 A third manner to obtain explicit examples of martingales $\left(M_{t}(F), t \geq 0\right)$.

- We begin with a definition. We shall say that a family of r.v.'s $\left(F_{t}, t \geq 0\right)$ converges, as $t \rightarrow \infty$, towards $F \mathbf{W}$ a.s. if for some $G>0, G \in L_{+}^{1}\left(\mathcal{F}_{\infty}, \mathbf{W}\right) F_{t} \underset{t \rightarrow \infty}{\longrightarrow} F W^{G}$ a.s. We recall : $W^{G}(\Gamma):=\mathbf{W}(G \Gamma), \Gamma \in b\left(\mathcal{F}_{\infty}\right)$. Clearly, this definition does not depend on the r.v. $G$ chosen in the above class. In particular, it may be convenient to take for $G$ the r.v. $\exp \left(-\frac{1}{2} A_{\infty}^{(q)}\right)$ for some $q \in \mathcal{I}$; hence, the a.s. $\mathbf{W}$-convergence is precisely the $W_{\infty}^{(q)}$ a.s. convergence.

This definition may seem complicated. However, its aim is to take care of the difficulty arising from the fact that for every $\Gamma_{t} \in b_{+}\left(F_{t}\right), \mathbf{W}\left(\Gamma_{t}\right)$ equals either 0 or $+\infty$ (see point $v$ ) of the proof of Theorem 1.1.2). 
Equivalently, $F_{t} \underset{t \rightarrow \infty}{\longrightarrow} F \quad \mathbf{W}$ a.s. if and only if $\mathbf{W}(\Delta)=0$ with $\Delta=\left\{\omega ; F_{t}(\omega) \underset{t \rightarrow \infty}{\underset{\longrightarrow}{\longrightarrow}} F(\omega)\right\}$

- In section 1.2.3 below we shall obtain the following result : (it is a Corollary of Theorem 1.2.5, in the same section 1.2.3)

Corollary 1.2.6. A positive $\left(\left(\mathcal{F}_{t}, t \geq 0\right), W\right)$ martingale $M_{t}, t \geq 0$ is of the form $\left(M_{t}(F)\right.$, $t \geq 0)$ for some $F \in L_{+}^{1}\left(\mathcal{F}_{\infty}, \mathbf{W}\right)$ if and only if :

$$
M_{0}=\mathbf{W}\left(\lim _{t \rightarrow \infty} \frac{M_{t}}{1+\left|X_{t}\right|}\right)
$$

and, in this case:

$$
F=\lim _{t \rightarrow \infty} \frac{M_{t}}{1+\left|X_{t}\right|} \quad \mathbf{W} \text { a.s. }
$$

- We now illustrate with 3 examples how due to this Corollary, we may compute explicitly $\left(M_{t}(F), t \geq 0\right)$ for some $F \in L_{+}^{1}\left(\mathcal{F}_{\infty}, \mathbf{W}\right)$.

Back to Example 1. Let $q \in \mathcal{I}$ and $M_{t}:=\varphi_{q}\left(X_{t}\right) \exp \left(-\frac{1}{2} A_{t}^{(q)}\right)$. Since

$$
\varphi_{q}(x) \underset{|x| \rightarrow \infty}{\sim}|x| \text { and }\left|X_{t}\right| \underset{t \rightarrow \infty}{\longrightarrow} \infty \quad \mathbf{W} \text { a.s. }
$$

we have :

$$
\frac{M_{t}}{1+\left|X_{t}\right|} \underset{t \rightarrow \infty}{\longrightarrow} \exp \left(-\frac{1}{2} A_{\infty}^{(q)}\right):=F
$$

On the other hand,

$$
M_{0}=\varphi_{q}(0)=\mathbf{W}\left(\exp \left(-\frac{1}{2} A_{\infty}^{(q)}\right)\right) \quad(\text { from }(1.1 .17))
$$

Thus, from Corollary 1.2.6. :

$$
M_{t}\left(\exp \left(-\frac{1}{2} A_{\infty}^{(q)}\right)\right)=\varphi_{q}\left(X_{t}\right) \exp \left(-\frac{1}{2} A_{t}^{(q)}\right)
$$

Back to Example 2. Let $h: \mathbb{R}_{+} \rightarrow \mathbb{R}_{+}$Borel and integrable and :

$$
M_{t}:=h\left(L_{t}\right)\left|X_{t}\right|+\int_{L_{t}}^{\infty} h(y) d y
$$

It is clear that :

$$
\frac{M_{t}}{1+\left|X_{t}\right|} \underset{t \rightarrow \infty}{\longrightarrow} h\left(L_{\infty}\right) \quad \mathbf{W} \text { a.s. }
$$

and that from (1.2.21) :

$$
M_{0}=\int_{0}^{\infty} h(y) d y=\mathbf{W}\left(h\left(L_{\infty}\right)\right)
$$


Thus, from Corollary 1.2.6:

$$
M_{t}\left(h\left(L_{\infty}\right)\right)=h\left(L_{t}\right)\left|X_{t}\right|+\int_{L_{t}}^{\infty} h(y) d y
$$

Back to Example 3. Let $\psi: \mathbb{R}_{+} \rightarrow \mathbb{R}_{+}$Borel and integrable, with $\psi(\infty)=0$. Let

$$
M_{t}:=\psi\left(S_{t}\right)\left(S_{t}-X_{t}\right)+\int_{S_{t}}^{\infty} \psi(y) d y
$$

Then :

$$
\frac{M_{t}}{1+\left|X_{t}\right|} \underset{t \rightarrow \infty}{\longrightarrow} \psi\left(S_{\infty}\right) \quad \text { W a.s. }(\text { see }(1.1 .99))
$$

Hence, from (1.2.22) :

$$
\mathbf{W}\left(\psi\left(S_{\infty}\right)\right)=\int_{0}^{\infty} \psi(l) d l=M_{0}
$$

Thus :

$$
M_{t}\left(\psi\left(S_{\infty}\right)\right)=\psi\left(S_{t}\right)\left(S_{t}-X_{t}\right)+\int_{S_{t}}^{\infty} \psi(y) d y
$$

\subsubsection{A decomposition theorem of positive Brownian supermartingales.}

Here is the most inportant result of this Section 1.2.

Theorem 1.2.5. Let $\left(Z_{t}, t \geq 0\right)$ denote a positive $\left(\left(\mathcal{F}_{t}, t \geq 0\right), W\right)$ supermartingale. We denote $Z_{\infty}:=\lim _{t \rightarrow \infty} Z_{t} \quad(W$ a.s. $)$. Then :

$$
\begin{gathered}
z_{\infty}:=\lim _{t \rightarrow \infty} \frac{Z_{t}}{1+\left|X_{t}\right|} \quad \text { exists } \mathbf{W} \text { a.s. } \\
\quad \text { and } \quad \mathbf{W}\left(z_{\infty}\right)<\infty
\end{gathered}
$$

2) $\left(Z_{t}, t \geq 0\right)$ decomposes in a unique manner in the form :

$$
Z_{t}=M_{t}\left(z_{\infty}\right)+W\left(Z_{\infty} \mid \mathcal{F}_{t}\right)+\xi_{t}, \quad t \geq 0
$$

where $\left(M_{t}\left(z_{\infty}\right), t \geq 0\right)$ and $\left(W\left(Z_{\infty} \mid \mathcal{F}_{t}\right), t \geq 0\right)$ denote two $\left(\left(\mathcal{F}_{t}, t \geq 0\right), W\right)$ martingales and :

$\left(\xi_{t}, t \geq 0\right)$ is a $\left(\left(\mathcal{F}_{t}, t \geq 0\right), W\right)$ positive supermartingale

such that:

i) $Z_{\infty} \in L_{+}^{1}\left(\mathcal{F}_{\infty}, W\right)$, hence $W\left(Z_{\infty} \mid \mathcal{F}_{t}\right)$ converges $W$ a.s. and in $L^{1}\left(\mathcal{F}_{\infty}, W\right)$ towards $Z_{\infty}$.

ii) $\quad \frac{W\left(Z_{\infty} \mid \mathcal{F}_{t}\right)+\xi_{t}}{1+\left|X_{t}\right|} \longrightarrow_{t \rightarrow \infty} 0 \quad \mathbf{W}$ a.s.

iii) $\quad M_{t}\left(z_{\infty}\right)+\xi_{t} \longrightarrow_{t \rightarrow \infty} 0 \quad W$ a.s.

After proving Theorem 1.2.5, we shall give a number of examples of $\left(\left(\mathcal{F}_{t}, t \geq 0\right), W\right)$ supermartingales for which we can compute explicitely the decomposition (1.2.52). 
We refer the reader to subsection 1.2.2.3 for the definition of the a.s. $\mathbf{W}$ convergence.

Corollary 1.2.6. (Characterisation of martingales of the form $\left(M_{t}(F), t \geq 0\right)$ ).

$A\left(\left(\mathcal{F}_{t}, t \geq 0\right), W\right)$ positive martingale $\left(Z_{t}, t \geq 0\right)$ is equal to $\left(M_{t}(F), t \geq 0\right)$ for an $F \in L_{+}^{1}\left(\mathcal{F}_{\infty}, \mathbf{W}\right)$ if and only if :

$$
Z_{0}=\mathbf{W}\left(\lim _{t \rightarrow \infty} \frac{Z_{t}}{1+\left|X_{t}\right|}\right)
$$

Note that $\lim _{t \rightarrow \infty} \frac{Z_{t}}{1+\left|X_{t}\right|}$ exists $\mathbf{W}$ a.s. from (1.2.50).

\section{Proof of Corollary 1.2.6.}

We write, from (1.2.52) :

$$
Z_{t}=M_{t}\left(z_{\infty}\right)+W\left(Z_{\infty} \mid \mathcal{F}_{t}\right)+\xi_{t}
$$

(where, in this situation, $\left(\xi_{t}, t \geq 0\right)$ is a positive martingale). Hence :

$$
Z_{0}=W\left(M_{0}\left(Z_{\infty}\right)\right)+W\left(W\left(z_{\infty} \mid \mathcal{F}_{0}\right)\right)+W\left(\xi_{0}\right)
$$

i.e., from (1.2.55) and (1.2.2) :

$$
Z_{0}=\mathbf{W}\left(z_{\infty}\right)=\mathbf{W}\left(z_{\infty}\right)+W\left(Z_{\infty}\right)+W\left(\xi_{0}\right)
$$

hence :

$$
W\left(Z_{\infty}\right)=W\left(\xi_{0}\right)=0 \quad \text { and } \quad W\left(Z_{\infty} \mid \mathcal{F}_{t}\right)=\xi_{t}=0, \quad \text { i.e. } Z_{t}=M_{t}\left(Z_{\infty}\right)
$$

\section{Proof of Theorem 1.2.5.}

This proof hinges on the three following Lemmas.

Lemma 1.2.7. Let $F, G \in L_{+}^{1}\left(\mathcal{F}_{\infty}, \mathbf{W}\right)$ and $G>0 \quad \mathbf{W}$ a.s. Then :

$$
\frac{M_{t}(F)}{M_{t}(G)}=W^{G}\left(\frac{F}{G} \mid \mathcal{F}_{t}\right) \quad W^{G} \text { a.s. }
$$

Consequently :

$$
\frac{M_{t}(F)}{M_{t}(G)} \underset{t \rightarrow \infty}{\longrightarrow} \frac{F}{G} \quad W^{G} \text { a.s. (hence } \mathbf{W} \text { a.s.) }
$$

Lemma 1.2.8. Let $F \in L_{+}^{1}\left(\mathcal{F}_{\infty}, \mathbf{W}\right)$. Then :

$$
\frac{M_{t}(F)}{1+\left|X_{t}\right|} \underset{t \rightarrow \infty}{\longrightarrow} F \quad \text { W a.s. }
$$

Lemma 1.2.9. Let $\left(Z_{t}, t \geq 0\right)$ denote a positive $\left(\left(\mathcal{F}_{t}, t \geq 0\right), W\right)$ supermartingale. Then : 1) $z_{\infty}:=\lim _{t \rightarrow \infty} \frac{Z_{t}}{1+\left|X_{t}\right|} \quad$ exists $\mathbf{W}$ a.s.

Furthermore :

$$
\mathbf{W}\left(z_{\infty}\right)<\infty
$$

2) For every $t \geq 0: \quad M_{t}\left(z_{\infty}\right) \leq Z_{t} \quad W$ a.s. 


\section{Proof of Lemma 1.2.7.}

We have, for every $t \geq 0$ and $\Gamma_{t} \in b\left(\mathcal{F}_{t}\right)$ :

$$
\begin{array}{rlrl}
W^{G}\left(\Gamma_{t} \frac{M_{t}(F)}{M_{t}(G)}\right) & =\mathbf{W}\left(\Gamma_{t} G \frac{M_{t}(F)}{M_{t}(G)}\right) & & \text { (by definition of } \left.W^{G}\right) \\
& =W\left(\Gamma_{t} M_{t}(G) \frac{M_{t}(F)}{M_{t}(G)}\right) & & \text { (by definition of } \left.M_{t}(G)\right) \\
& =W\left(\Gamma_{t} M_{t}(F)\right) & & \text { (by definition of } \left.M_{t}(F)\right) \\
& =\mathbf{W}\left(\Gamma_{t} F\right) & & \text { (by definition of } \left.W^{G}\right) \\
& =W^{G}\left(\Gamma_{t} \frac{F}{G}\right) & & \\
& =W^{G}\left(\Gamma_{t} W^{G}\left(\frac{F}{G} \mid \mathcal{F}_{t}\right)\right) &
\end{array}
$$

This is (1.2.56). Now (1.2.57) is an immediate consequence of (1.2.56) since $\frac{F}{G} \in L^{1}\left(W^{G}\right)$. Indeed : $W^{G}\left(\frac{F}{G}\right)=\mathbf{W}\left(G \cdot \frac{F}{G}\right)=\mathbf{W}(F)<\infty$.

\section{Proof of Lemma 1.2.8.}

i) We first apply Lemma 1.2 .7 with $G:=\exp \left(-\frac{1}{2} A_{\infty}^{(q)}\right)$, for any $q \in \mathcal{I}$. Then, recall that (Example 1) $M_{t}(G)=\varphi_{q}\left(X_{t}\right) \exp \left(-\frac{1}{2} A_{t}^{(q)}\right)$ and, since $\varphi_{q}(x) \sim|x|$ as $|x| \rightarrow \infty$, we get :

$$
\frac{M_{t}(G)}{1+\left|X_{t}\right|} \underset{t \rightarrow \infty}{\longrightarrow} \exp \left(-\frac{1}{2} A_{\infty}^{(q)}\right)=G \quad \text { W a.s. }
$$

which is the statement of Lemma 1.2.8 with $F=\exp \left(-\frac{1}{2} A_{\infty}^{(q)}\right)$.

ii) For a general $F \in L_{+}^{1}\left(\mathcal{F}_{\infty}, \mathbf{W}\right)$, we write :

$$
\frac{M_{t}(F)}{1+\left|X_{t}\right|}=\frac{M_{t}(F)}{M_{t}(G)} \cdot \frac{M_{t}(G)}{1+\left|X_{t}\right|} \underset{t \rightarrow \infty}{\longrightarrow} \frac{F}{G} \cdot G \quad \text { W a.s. }
$$

by applying Lemma 1.2.7, and the result of point $i$ ) above.

Proof of Lemma 1.2.9.

i) We begin with an argument similar to the one we used to prove Lemma 1.2.8, that is : we write :

$$
\frac{Z_{t}}{1+\left|X_{t}\right|}=\frac{Z_{t}}{M_{t}(G)} \frac{M_{t}(G)}{1+\left|X_{t}\right|}
$$

We now use the fact that $\left(\frac{Z_{t}}{M_{t}(G)}, t \geq 0\right)$ is a $\left(\left(\mathcal{F}_{t}, t \geq 0\right), W^{G}\right)$ supermartingale (positive) ; hence it converges $W^{G}$ a.s. to a r.v. $\zeta$; consequently:

$$
z_{\infty}:=\lim _{t \rightarrow \infty} \frac{Z_{t}}{1+\left|X_{t}\right|} \quad \text { exists } W^{G} \text { a.s. }
$$


and we have :

$$
z_{\infty}=\zeta \cdot G
$$

ii) since $\zeta:=\lim _{t \rightarrow \infty} \frac{Z_{t}}{M_{t}(G)}, W^{G}$ a.s., is the limit as $t \rightarrow \infty$ of a $W^{G}$ supermartingale, we have :

$$
\begin{aligned}
& W^{G}(\zeta) \leq \frac{Z_{0}}{M_{0}(G)} \quad \text { hence : } \\
& \mathbf{W}\left(z_{\infty}\right)=W^{G}(\zeta) \leq \frac{Z_{0}}{M_{0}(G)}<\infty
\end{aligned}
$$

iii) For any $t \geq 0$ and $\Gamma_{t} \in b_{+}\left(\mathcal{F}_{t}\right)$, we have :

$$
\begin{aligned}
\mathbf{W}\left(\Gamma_{t} z_{\infty}\right) & =\mathbf{W}\left(\Gamma_{t} \lim _{u \rightarrow \infty} \frac{Z_{u}}{1+\left|X_{u}\right|}\right) \\
& =\mathbf{W}\left(\Gamma_{t} \lim _{u \rightarrow \infty} \frac{Z_{u}}{1+\left|X_{u}\right|} \cdot 1_{g \leq u}\right) \\
& \leq \varliminf_{u \rightarrow \infty} \mathbf{W}\left(\Gamma_{t} \frac{Z_{u}}{1+\left|X_{u}\right|} 1_{g \leq u}\right) \quad \text { (from Fatou's Lemma) } \\
& =\varliminf_{u \rightarrow \infty} W\left(\Gamma_{t} \frac{Z_{u}}{1+\left|X_{u}\right|}\left|X_{u}\right|\right) \quad \text { (from point } 2 \mathbf{i} \text { ) of Theorem 1.1.6) } \\
& \leq \varliminf_{u \rightarrow \infty} W\left(\Gamma_{t} Z_{u}\right) \quad\left(\text { since } \frac{\left|X_{u}\right|}{1+\left|X_{u}\right|} \leq 1\right) \\
& \leq W\left(\Gamma_{t} Z_{t}\right)
\end{aligned}
$$

since $\left(Z_{t}, t \geq 0\right)$ is a supermartingale. Finally :

$$
\mathbf{W}\left(\Gamma_{t} z_{\infty}\right)=W\left(\Gamma_{t} M_{t}\left(z_{\infty}\right)\right) \leq W\left(\Gamma_{t} \cdot Z_{t}\right)
$$

which is equivalent to point 2 of Lemma 1.2.9.

We may now end the proof of Theorem 1.2.5.

Let $\quad \widetilde{Z}_{t}:=Z_{t}-M_{t}\left(z_{\infty}\right) \quad(t \geq 0)$

Since $\left(M_{t}\left(z_{\infty}\right), t \geq 0\right)$ is a $\left(\left(\mathcal{F}_{t}, t \geq 0\right), W\right)$ martingale, the process $\left(\widetilde{Z}_{t}, t \geq 0\right)$ is still a $\left(\left(\mathcal{F}_{t}, t \geq 0\right), W\right)$ positive (from (1.2.61)) supermartingale, and since $M_{t}\left(z_{\infty}\right) \underset{t \rightarrow \infty}{\longrightarrow} \quad W$ a.s. from Theorem 1.2.1, we obtain :

$$
\widetilde{Z}_{t} \underset{t \rightarrow \infty}{\longrightarrow} Z_{\infty} \quad W \text { a.s. }
$$

Since $\left(\widetilde{Z}_{t}, t \geq 0\right)$ is a positive supermartingale, we obtain :

$$
W\left(Z_{\infty} \mid \mathcal{F}_{t}\right) \leq \widetilde{Z}_{t}
$$

We now write :

$$
\xi_{t}:=\widetilde{Z}_{t}-W\left(Z_{\infty} \mid \mathcal{F}_{t}\right) \quad t \geq 0
$$


This is a positive supermartingale such that $\lim _{t \rightarrow \infty} \xi_{t}=0 \quad W$ a.s. On the other hand, $\mathbf{W}$ a.s. :

$$
\lim _{t \rightarrow \infty} \frac{\xi_{t}}{1+\left|X_{t}\right|}=\lim _{t \rightarrow \infty} \frac{\widetilde{Z}_{t}}{1+\left|X_{t}\right|}=z_{\infty}-z_{\infty}=0
$$

The uniqueness of decomposition (1.2.52) being immediate, Theorem 1.2.5 is proven.

1.2.4 A decomposition result of the martingale $\left(M_{t}(F), t \geq 0\right)$.

A difference with the preceding subsection is that the r.v.'s $F$ which we now consider belong to $L^{1}\left(\mathcal{F}_{\infty}, W\right)$, but are not necessarily positive.

We shall now prove a decomposition result of the $\left(\left(\mathcal{F}_{t}, t \geq 0\right), W\right)$ martingale $\left(M_{t}(F), t \geq\right.$ $0)$. For this purpose, we shall use the following lemma.

Lemma 1.2.10. Let $F \in L^{1}\left(\mathcal{F}_{\infty}, \mathbf{W}\right)$

1) There exists a predictable process $\left(k_{s}(F), s \geq 0\right)$ which is defined $d L_{s}(\omega) W(d \omega)$ a.s., and is positive if $F$ is positive, such that:

$$
W\left(\int_{0}^{\infty}\left|k_{s}(F)\right| d L_{s}\right)=\mathbf{W}\left(\left|k_{g}(F)\right|\right) \leq \mathbf{W}(|F|)<\infty
$$

and such that for every bounded predictable process $\left(\Phi_{s}, s \geq 0\right)$

$$
\begin{aligned}
\mathbf{W}\left(\Phi_{g} F\right) & =W\left(\int_{0}^{\infty} \Phi_{s} k_{s}(F) d L_{s}\right) \\
& =\mathbf{W}\left(\Phi_{s} k_{g}(F)\right) \\
\text { Thus }: \quad \mathbf{W}\left(F \mid \mathcal{F}_{g}\right) & =k_{g}(F)
\end{aligned}
$$

2) We have $\mathbf{W}\left(\left|k_{g}(F)\right|\right)<\infty$ (from (1.2.62))

$$
\mathbf{W}\left(\left|k_{g}(F)\right|\right) \leq \mathbf{W}(|F|)<\infty
$$

and

$$
\left(k_{s}\left(k_{g}(F), s \geq 0\right)=\left(k_{s}(F), s \geq 0\right) \quad d L_{s}(\omega) W(d \omega) \quad\right. \text { a.s. }
$$

3) If $\left(h_{s}, s \geq 0\right)$ is a predictable process such that $\mathbf{W}\left(\left|h_{g}\right|\right)<\infty$, then:

$$
\left(k_{s}\left(h_{g}\right), s \geq 0\right)=\left(h_{s}, s \geq 0\right) \quad d L_{s}(\omega) W(d \omega) \quad \text { a.s. }
$$

\section{Proof of Lemma 1.2.10.}

It suffices, by linearity, to prove this Lemma when $F \geq 0$.

i) Formula (1.2.64), written for $F \equiv 1$ and $k_{s}(F) \equiv 1$ :

$$
\mathbf{W}\left(\Phi_{g}\right)=W\left(\int_{0}^{\infty} \Phi_{s} d L_{s}\right)
$$

is formula (1.1.44). Let us define the measure $\mu_{F}$, on the predictable $\sigma$-field, and more generally on the set of positive predictable processes by :

$$
\mu_{F}(\Phi)=\mathbf{W}\left(\Phi_{g} \cdot F\right)
$$


Clearly, $\mu_{F}$ is absolutely continuous, on the predictable $\sigma$-field, with respect to $\mu_{1}$, which is the measure $\mu_{F}$ for $F \equiv 1$. Thus, from (1.2.69), $\mu_{F}$ is absolutely continuous on the predictable $\sigma$-field with respect to the measure $d L_{s}(\omega) W(d \omega)$. Thus, there exists, from the Radon-Nikodym Theorem, a process $\left(k_{s}(F), s \geq 0\right)$ which is predictable such that, for every $\Phi \geq 0$ predictable :

$$
\mu_{F}(\Phi)=\mathbf{W}\left(\Phi_{g} \cdot F\right)=W\left(\int_{0}^{\infty} \Phi_{s} k_{s}(F) d L_{s}\right)
$$

This is relation (1.2.64). The further relations (1.2.65) and (1.2.66) follow immediately.

ii) The other points of Lemma 1.2.10 are elementary. We show, for example, (1.2.68). We have, from (1.2.63) and 1.2.69), for every predictable and bounded process $\Phi$ :

$$
\begin{aligned}
\mathbf{W}\left(\Phi_{g} h_{g}\right) & =W\left(\int_{0}^{\infty} \Phi_{s} k_{s}\left(h_{g}\right) d L_{s}\right) \\
& =W\left(\int_{0}^{\infty} \Phi_{s} h_{s} d L_{s}\right)
\end{aligned}
$$

Hence, $\Phi$ being arbitrary, (1.2.68). Relation (1.2.67) is obtained by application of (1.2.68) with $\left(h_{s}, s \geq 0\right)=\left(k_{s}(F), s \geq 0\right)$.

Here is now the announced decomposition Theorem.

Theorem 1.2.11. Let $F \in L^{1}\left(\mathcal{F}_{\infty}, \mathbf{W}\right)$. There exist two continuous positive processes $\left(\Sigma_{t}(F), t \geq 0\right)$ and $\left(\Delta_{t}(F), t \geq 0\right)$ such that, for every $t \geq 0$ :

$$
M_{t}(F)=\Sigma_{t}(F)+\Delta_{t}(F) \quad(t \geq 0)
$$

with :

1) i) For every $t \geq 0$ and $\Gamma_{t} \in b\left(\mathcal{F}_{t}\right)$ :

$$
\mathbf{W}\left(\Gamma_{t} 1_{g \leq t} F\right)=W\left(\Gamma_{t} \Sigma_{t}(F)\right)
$$

ii) $\left(\Sigma_{t}(F), t \geq 0\right)$ is a quasimartingale (a submartingale if $F \geq 0$ ) which vanishes on the zero set of $\left(X_{u}, u \geq 0\right)$. Its Doob-Meyer decomposition writes :

$$
\Sigma_{t}(F)=-M_{t}^{\Sigma(F)}+\int_{0}^{t} k_{s}(F) d L_{s}
$$

In particular, the bounded variation part of this decomposition is absolutely continuous with respect to $d L_{s}$. In (1.2.73), $\left(M_{t}^{\Sigma(F)}, t \geq 0\right)$ is a $\left(\left(\mathcal{F}_{t}, t \geq 0\right), W\right)$ martingale satisfying, if $F \geq 0$ :

$$
\begin{aligned}
& \sup _{s \leq t} M_{s}^{\Sigma(F)}=\int_{0}^{t} k_{s}(F) d L_{s} \\
& \lim _{t \rightarrow \infty} M_{t}^{\Sigma(F)}:=M_{\infty}^{\Sigma(F)}=\int_{0}^{\infty} k_{s}(F) d L_{s}=\sup _{t \geq 0} M_{t}^{\Sigma(F)}
\end{aligned}
$$

In particular, this martingale is not uniformly integrable.

iii) We have the "explicit formula" :

$$
\Sigma_{t}(F)=\left|X_{t}\right| \cdot \widehat{E}_{X_{t}}^{(3)}\left(F\left(\omega_{t}, \widehat{\omega}_{t}\right)\right)
$$


(see point 1 of Remark 1.2.2 for such a notation).

In (1.2.76), the expectation is taken with respect to $\widehat{\omega}_{t}$, the letter $\omega_{t}$, and $X_{t}$, being frozen ; $\widehat{E}_{X_{+}}^{(3)}$ denotes the expectation relatively to a 3-dimensional Bessel process starting from $X_{t}$, if $X_{t}>0$, and the expectation with respect to the opposite of a 3-dimensional Bessel process, if $X_{t}<0$.

iv) The application $F \rightarrow\left(\Sigma_{t}(F), t \geq 0\right)$ is injective since :

$$
\frac{\Sigma_{t}(F)}{1+\left|X_{t}\right|} \underset{t \rightarrow \infty}{\longrightarrow} F \quad \text { W a.s. }
$$

v) We have, for every $t \geq 0$ :

$$
W\left\{\left(\Sigma_{t}(F)-\Sigma_{t}\left(k_{g}(F)\right) \mid \mathcal{F}_{g^{(t)}}\right)=0\right.
$$

2)i) For every $t \geq 0$ and $\Gamma_{t} \in b\left(\mathcal{F}_{t}\right)$ :

$$
\mathbf{W}\left(\Gamma_{t} 1_{g>t} F\right)=W\left(\Gamma_{t} \Delta_{t}(F)\right)
$$

ii) $\left(\Delta_{t}(F), t \geq 0\right)$ is a quasimartingale (a positive supermartingale if $F \geq 0$ ). Its DoobMeyer decomposition writes :

$$
\Delta_{t}(F)=M_{t}^{\Delta(F)}-\int_{0}^{t} k_{s}(F) d L_{s}
$$

where $\left(M_{t}^{\Delta(F)}, t \geq 0\right)$ is the $\left(\left(\mathcal{F}_{t}, t \geq 0\right), \quad W\right)$ martingale given by :

$$
M_{t}^{\Delta(F)}=W\left(\int_{0}^{\infty} k_{s}(F) d L_{s} \mid \mathcal{F}_{t}\right)
$$

In particular, since from (1.2.62), $\int_{0}^{\infty} k_{s}(F) d L_{s} \in L^{1}\left(\mathcal{F}_{\infty}, W\right)$, this martingale is uniformly integrable.

iii) The application $F \rightarrow\left(\Delta_{t}(F), t \geq 0\right)$ is not injective since :

$$
\left(\Delta_{t}(F), t \geq 0\right)=\left(\Delta_{t}\left(k_{g}(F)\right), t \geq 0\right)
$$

$\left(\right.$ and $k_{g}(F) \neq F$ when $F$ is not $\mathcal{F}_{g}$ measurable $)$.

3)i) The martingale $\left(M_{t}(F), t \geq 0\right)$ satisfies to:

$$
\left(W\left(M_{t}(F) \mid \mathcal{F}_{g^{(t)}}\right), t \geq 0\right)=\left(W\left(M_{t}\left(k_{g}(F)\right) \mid \mathcal{F}_{g^{(t)}}\right), t \geq 0\right)
$$

The following Theorem is an important consequence of Theorem 1.2.11. 
Theorem 1.2.12. Let $F \in L^{1}\left(\mathcal{F}_{\infty}, \mathbf{W}\right)$.

Then, the $\left(\left(\mathcal{F}_{t}, t \geq 0\right), W\right)$ martingale $\left(M_{t}(F), t \geq 0\right)$ vanishes on the zeros of $\left(X_{u}, u \geq 0\right)$ if and only if $k_{g}(F)=0$.

\section{Remark 1.2.13}

1) If $F=F_{g}$, with $\left(F_{u}, u \geq 0\right)$ a positive previsible process Theorem 1.2 .3 implies, in this particular case :

$$
\Sigma_{t}\left(F_{g}\right)=F_{g^{(t)}} \cdot\left|X_{t}\right|, \quad \Delta_{t}\left(F_{g}\right)=\int_{L_{t}}^{\infty} W\left(F_{\tau_{l}} \mid \mathcal{F}_{t}\right) d l .
$$

2) If $F \geq 0$, the supermartingale $\left(\Delta_{t}(F), t \geq 0\right)$ satisfies :

$$
\Delta_{t}(F) \underset{t \rightarrow \infty}{\longrightarrow} 0 \quad W \text { a.s., } \quad \text { since } 0 \leq \Delta_{t}(F) \leq M_{t}(F)
$$

and

$$
\frac{\Delta_{t}(F)}{1+\left|X_{t}\right|}=\frac{M_{t}(F)}{1+\left|X_{t}\right|}-\frac{\Sigma_{t}(F)}{1+\left|X_{t}\right|} \underset{t \rightarrow \infty}{\longrightarrow} F-F=0 \quad \text { W a.s. }
$$

from Lemma 1.2.8 and (1.2.77). Hence, in the decomposition (1.2.52) of the supermartingale $\Delta_{t}(F)$, there remains uniquely the term $\left(\xi_{t}, t \geq 0\right)$.

3) When $F \geq 0$, gathering the terms (1.2.71), (1.2.73), (1.2.80) and (1.2.81), we have :

$$
M_{t}(F)=-M_{t}^{\Sigma(F)}+W\left(\int_{0}^{\infty} k_{s}(F) d L_{s} \mid \mathcal{F}_{t}\right)
$$

This formula implies (from (1.2.75)) that $\left(M_{t}^{\Sigma(F)}, t \geq 0\right)$ is not uniformly integrable since if were, then $\left(M_{t}(F), t \geq 0\right)$ would be null.

4) From relation (1.2.83) there exists an application

$$
\begin{aligned}
m: \quad L^{1}(\mathcal{F}, \mathbf{W}) & \longrightarrow \mathcal{M}\left(\left(\mathcal{F}_{g^{(t)}}, t \geq 0\right), \quad W\right) \\
F & \longrightarrow\left(m_{t}(F), t \geq 0\right)
\end{aligned}
$$

where $\mathcal{M}\left(\left(\mathcal{F}_{g^{(t)}}, t \geq 0\right), W\right)$ denotes the set of $\left(\left(\mathcal{F}_{g^{(t)}}, t \geq 0\right), W\right)$ martingales ; this application $m$ is such that :

$$
m_{t}(F)=W\left(M_{t}\left(k_{g}(F)\right) \mid \mathcal{F}_{g^{(t)}}\right)
$$

with

$$
m_{t}(F):=\sigma_{t}(F)+\delta_{t}(F)
$$

and

$$
\begin{aligned}
\sigma_{t}(F) & =\sqrt{\frac{\pi}{2}} k_{g^{(t)}}(F) \sqrt{t-g^{(t)}} \\
\delta_{t}(F) & =W\left(\int_{t}^{\infty} k_{s}(F) d L_{s} \mid \mathcal{F}_{g^{(t)}}\right)
\end{aligned}
$$


If $F \geq 0,\left(\sigma_{t}(F), t \geq 0\right)$ resp. $\left(\delta_{t}(F), t \geq 0\right)$ is a $\left(\left(\mathcal{F}_{g^{(t)}}, t \geq 0\right), \quad W\right)$ submartingale (resp. $\left(\left(\mathcal{F}_{g^{(t)}}, t \geq 0\right), \quad W\right)$ supermartingale.

5) We recall that by definition, a process $\left(Z_{t}, t \geq 0\right)$ is a quasimartingale if, for every $t \geq 0$ :

$$
\sup W\left(\sum_{i=1}^{n-1}\left|W\left(Z_{t_{i+1}}-Z_{t_{i}}\right)\right| \mathcal{F}_{t_{i}}\right)<\infty
$$

the sup being taken over the set of subdivisions $0 \leq t_{1}<\cdots<t_{n}<t$. In fact, such a process is the difference of two supermartingales (see [Rao]). On the other hand, the Föllmer measure (see [Föl] $\mu_{Z}$ - with finite mass - of a supermartingale $\left(Z_{t}, t \geq 0\right)$ (or of a quasimartingale) is the measure defined on the predictable $\sigma$-field and characterised by :

$$
\mu_{Z}\left(\Gamma_{t} 1_{] t, \infty]}\right)=W\left(\Gamma_{t} \cdot Z_{t}\right) \quad\left(\Gamma_{t} \in b\left(\mathcal{F}_{t}\right)\right)
$$

Hence formulae (1.2.65) and (1.2.79) imply that the measure $\mu_{F}$ defined by $(1.2 .70)$ is the Föllmer measure of the quasimartingale $\left(\Delta_{t}(F), t \geq 0\right)$.

Proof of Theorem 1.2.11.

i) We define $\Sigma_{t}(F)$ via :

$$
\Sigma_{t}(F)=\left.M_{t}\left(F 1_{g \leq a}\right)\right|_{a=t}
$$

Hence, for every $\Gamma_{t} \in b\left(\mathcal{F}_{t}\right)$ :

$$
\mathbf{W}\left(\Gamma_{t} 1_{g \leq t} \cdot F\right)=W\left(\Gamma_{t} \Sigma_{t}(F)\right)
$$

It is easy to deduce from (1.2.86) that $\left(\Sigma_{t}(F)=\Sigma_{t}\left(F^{+}\right)-\Sigma_{t}\left(F^{-}\right), t \geq 0\right)$ is a semimartingale, as the difference of two submartingales and we shall show below (see point vi) of this proof) that it is in fact a quasimartingale which admits a continuous version.

ii) We show (1.2.73).

By linearity, it suffices to prove (1.2.73) for $F \geq 0$.From (1.2.68), we have for $s \leq t$ and $\Gamma_{s} \in b\left(\mathcal{F}_{s}\right):$

$$
\begin{aligned}
\mathbf{W}\left(\Gamma_{s} 1_{s \leq g \leq t} F\right) & =W\left(\Gamma_{s}\left(\Sigma_{t}(F)-\Sigma_{s}(F)\right)\right) \\
& =W\left(\Gamma_{s} \cdot \int_{s}^{t} k_{u}(F) d L_{u}\right)
\end{aligned}
$$

by using Lemma 1.2.10 with $\left(\Phi_{u}:=\Gamma_{s} 1_{s s, t]}(u), u \geq 0\right)$ (1.2.73) follows immediately from (1.2.87).

iii) We show (1.2.74) and (1.2.75).

Since, if $F \geq 0$, then $\Sigma_{s}(F) \geq 0$, we have :

$$
\begin{aligned}
& \sup _{s \leq t} M_{s}^{\Sigma(F)} \leq \int_{0}^{t} k_{u}(F) d L_{u} \quad \text { and } \\
& \sup _{s \leq t} M_{s}^{\Sigma(F)} \geq \sup _{s \leq g^{(t)}} M_{s}^{\Sigma(F)}=\int_{0}^{g^{(t)}} k_{u}(F) d L_{u}=\int_{0}^{t} k_{u}(F) d L_{u}
\end{aligned}
$$

since $\Sigma_{g^{(t)}}(F)=0$ from $(1.2 .76)$. 
On the other hand, since $0 \leq \Sigma_{t}(F) \leq M_{t}(F)$ and since $M_{t}(F) \underset{t \rightarrow \infty}{\longrightarrow} 0 \quad W$ a.s. from Theorem 1.2.1, we have $\Sigma_{t}(F) \underset{t \rightarrow \infty}{\longrightarrow} \quad W$ a.s., and thus, from (1.2.73) :

$$
\lim _{t \rightarrow \infty} M_{t}^{\Sigma(F)}:=M_{\infty}^{\Sigma(F)}=\int_{0}^{\infty} k_{s}(F) d L_{s}=\sup _{t \geq 0} M_{t}^{\Sigma(F)}
$$

which, in particular, proves, that $\left(M_{t}^{\Sigma(F)}, t \geq 0\right)$ is not uniformly integrable.

iv) We show (1.2.76).

For this purpose, we shall use the notation and results of subsection 1.1.4. We have, for every $t \geq 0$ and $\Gamma_{t} \in b\left(\mathcal{F}_{t}\right):$

$$
\begin{aligned}
W\left(\Gamma_{t} \Sigma_{t}(F)\right) & \\
= & \mathbf{W}\left(\Gamma_{t} 1_{g \leq t} F\right) \quad(\text { from }(1.2 .86)) \\
= & 2 W_{\infty}^{\left(\delta_{0}\right)}\left(\Gamma_{t} 1_{g \leq t} F e^{\left.\frac{1}{2} L_{\infty}\right)}\right. \\
= & 2 W_{\infty}^{\left(\delta_{0}\right)}\left(\Gamma_{t} e^{\frac{1}{2} L_{t}} 1_{g \leq t} F\right) \quad\left(\text { since }: L_{\infty}=L_{t} \text { on the set }(g \leq t)\right) \\
= & 2 W_{\infty}^{\left(\delta_{0}\right)}\left(\Gamma_{t} e^{\frac{1}{2} L_{t}} W_{\infty}^{\left(\delta_{0}\right)}\left(1_{g \leq t} \cdot F \mid \mathcal{F}_{t}\right)\right) \\
= & 2 W_{\infty}^{\left(\delta_{0}\right)}\left(\Gamma_{t} e^{\frac{1}{2} L_{t}} W_{\infty}^{\left(\delta_{0}\right)}\left(1_{T_{0} \circ \theta_{t}=\infty} \cdot F \mid \mathcal{F}_{t}\right)\right) \quad\left(\text { since }(g \leq t)=\left(T_{0} \circ \theta_{t}=\infty\right)\right) \\
= & 2 W_{\infty}^{\left(\delta_{0}\right)}\left(\Gamma_{t} e^{\frac{1}{2} L_{t}} \widehat{W}_{X_{t}, \infty}^{\left(\delta_{0}\right)}\left(1_{T_{0}=\infty} F\left(\omega_{t}, \widehat{\omega}^{t}\right)\right)\right. \\
& (\text { by the Markov property) } \\
= & 2 W_{\infty}^{\left(\delta_{0}\right)}\left(\Gamma_{t} e^{\frac{1}{2} L_{t}} \widehat{W}_{X_{t}, \infty}^{\left(\delta_{0}\right)}\left(F\left(\omega_{t}, \widehat{\omega}^{t}\right) \mid T_{0}=\infty\right) \cdot W_{X_{t}, \infty}^{\left(\delta_{0}\right)}\left(T_{0}=\infty\right)\right)
\end{aligned}
$$

But, from (1.1.70) :

$$
W_{X_{t}, \infty}^{\left(\delta_{0}\right)}\left(T_{0}=\infty\right)=\frac{\left|X_{t}\right|}{2+\left|X_{t}\right|}
$$

and, from Theorem 1.1.5, conditionally on $\left(T_{0}=\infty\right), W_{\infty, x}^{\left(\delta_{0}\right)}$ is the law of a Bessel (3) process (resp. of the opposite of a Bessel (3) process) started at $x$ if $x>0$ (resp. if $x<0$ ). Then :

$$
\begin{aligned}
W\left(\Gamma_{t} \Sigma_{t}(F)\right) & =2 W_{\infty}^{\left(\delta_{0}\right)}\left(\Gamma_{t} e^{\frac{1}{2} L_{t}} \frac{\left|X_{t}\right|}{2+\left|X_{t}\right|} \widehat{E}_{X_{t}}^{(3)}\left(F\left(\omega_{t}, \widehat{\omega}^{t}\right)\right)\right) \\
& =W\left(\Gamma_{t} e^{\frac{1}{2} L_{t}} \frac{\left|X_{t}\right|}{2+\left|X_{t}\right|} \widehat{E}_{X_{t}}^{(3)}\left(F\left(\omega_{t}, \widehat{\omega}^{t}\right)\right) e^{\frac{1}{2} L_{t}}\left(2+\left|X_{t}\right|\right)\right)
\end{aligned}
$$

(from (1.1.31) and (1.1.7)).

Finally $W\left(\Gamma_{t} \Sigma_{t}(F)\right)=W\left(\Gamma_{t}\left|X_{t}\right| \widehat{E}_{X_{t}}^{(3)}\left(F\left(\omega_{t}, \widehat{\omega}^{t}\right)\right)\right)$

It is relation (1.2.76). Observe that this relation implies $\left(\Sigma_{t}(F), t \geq 0\right)$ vanishes on the zeros of $\left(X_{t}, t \geq 0\right)$. On the other hand, (1.2.76) implies (1.2.77), since, under $\mathbf{W},\left|X_{t}\right| \underset{t \rightarrow \infty}{\longrightarrow}$ a.s.

v) We show (1.2.83) and (1.2.78).

For every positive, bounded and predictable process $\left(\Phi_{u}, u \geq 0\right)$, we have :

$$
W\left(\Phi_{g^{(t)}} M_{t}(F)\right)=\mathbf{W}\left(\Phi_{g^{(t)}} \cdot F\right)
$$


by definition of $M_{t}(F)$. But, the $\sigma$-algebra $\mathcal{F}_{g^{(t)}}$ is contained in $\mathcal{F}_{g}$. Hence the RHS of (1.2.89) equals from $(1.2 .65)$ :

$$
\mathbf{W}\left(\Phi_{g^{(t)}} k_{g}(F)\right)=W\left(\Phi_{g^{(t)}} M_{t}\left(k_{g}(F)\right)\right)
$$

Finally :

$$
W\left(\Phi_{g^{(t)}} k_{g}(F)\right)=W\left(\Phi_{g^{(t)}} M_{t}\left(k_{g}(F)\right)\right)
$$

Thus $W\left(M_{t}(F)-M_{t}\left(k_{g}(F)\right) \mid \mathcal{F}_{g^{(t)}}\right)=0$ i.e. (1.2.83) is satisfied. (1.2.78) is proven by using the same arguments.

vi) We show (1.2.79).

We define $\Delta_{t}(F)$ by :

$$
\Delta_{t}(F):=\left.M_{t}\left(F 1_{g>a}\right)\right|_{a=t}
$$

It is clear that :

$$
M_{t}(F)=\Sigma_{t}(F)+\Delta_{t}(F)
$$

and that, for every $t \geq 0$ and $\Gamma_{t} \in b\left(\mathcal{F}_{t}\right)$ :

$$
\mathbf{W}\left(\Gamma_{t} 1_{g>t} F\right)=W\left(\Gamma_{t} \Delta_{t}(F)\right)
$$

Then writing $\Delta_{t}(F)=\Delta_{t}\left(F^{+}\right)-\Delta_{t}\left(F^{-}\right)$we deduce easily from this formula that $\left(\Delta_{t}^{ \pm}(F)\right.$, $t \geq 0)$ are two positive supermartingales and then $\left(\Delta_{t}\left(F^{+}\right), t \geq 0\right)$ is a quasimartingale. Since $\Sigma_{t}(F)=M_{t}(F)-\Delta_{t}(F)=M_{t}\left(F^{+}\right)-M_{t}\left(F^{-}\right)-\Delta_{t}\left(F^{+}\right)+\Delta_{t}\left(F^{-}\right)$, it is clear that $\left(\Sigma_{t}(F), t \geq 0\right)$ is still a quasimartingale. Formula (1.2.80) then results from (1.2.73) and (1.2.71). Finally, thanks to (1.2.80) and (1.2.73), $\left(\Delta_{t}(F), t \geq 0\right)$ and $\left(\Sigma_{t}(F), t \geq 0\right)$ admit continuous versions.

vii) We show (1.2.81).

We have, from (1.2.79), for every $\Gamma_{t} \in b\left(\mathcal{F}_{t}\right)$

$$
\begin{aligned}
\mathbf{W}\left(\Gamma_{t} 1_{g>t} F\right) & =W\left(\Gamma_{t} \Delta_{t}(F)\right) \\
& =\mathbf{W}\left(\widetilde{\Gamma}_{g} \cdot F\right) \\
\left(\text { with }\left(\widetilde{\Gamma}_{u}, u \geq 0\right)\right. & \left.:=\left(\Gamma_{t} 1_{t, \infty[}(u), u \geq 0\right)\right) \\
& =W\left(\int_{0}^{\infty} \widetilde{\Gamma}_{u} k_{u}(F) d L_{u}\right) \quad(\text { from Lemma 1.2.9) } \\
& =W\left(\Gamma_{t} \cdot \int_{t}^{\infty} k_{u}(F) d L_{u}\right) \\
& =W\left(\Gamma_{t} W\left(\int_{t}^{\infty} k_{u}(F) d L_{u} \mid \mathcal{F}_{t}\right)\right) \\
\text { Hence : } \Delta_{t}(F) & =W\left(\int_{t}^{\infty} k_{u}(F) d L_{u} \mid \mathcal{F}_{t}\right) \\
& =W\left(\int_{0}^{\infty} k_{u}(F) d L_{u} \mid \mathcal{F}_{t}\right)-\int_{0}^{t} k_{u}(F) d L_{u}
\end{aligned}
$$

This is relation $(1.2 .80)$ (and (1.2.81)). 
viii) We show (1.2.82).

It suffices, to prove (1.2.82), to show that for every $t \geq 0$ and $\Gamma_{t} \in b\left(\mathcal{F}_{t}\right)$, we have :

$$
W\left(\Gamma_{t} \Delta_{t}\left(F-k_{g}(F)\right)=0\right.
$$

But :

$$
\begin{aligned}
& W\left(\Gamma_{t} \Delta_{t}\left(F-k_{g}(F)\right)\right)=\mathbf{W}\left(\Gamma_{t} 1_{g>t}\left(F-k_{g}(F)\right)\right. \\
& =\mathbf{W}\left(\widetilde{\Gamma}_{g}\left(F-k_{g}(F)\right)\right. \\
& \left(\text { with } \quad\left(\widetilde{\Gamma}_{u}:=\Gamma_{t} 1_{], \infty[}(u), u \geq 0\right)\right) \\
& =W\left(\Gamma_{t} \int_{t}^{\infty}\left(k_{u}(F)-k_{u}\left(k_{g}(F)\right)\right) d L_{u}\right) \quad(\text { from (1.2.63)) } \\
& =0
\end{aligned}
$$

since $\quad k_{u}(F)=k_{u}\left(k_{g}(F)\right)$, from (1.2.67).

ix) Observe that, by using (1.2.82), (1.2.83) is a consequence of (1.2.78). Indeed :

$$
\begin{array}{rlr}
M_{t}\left(F-k_{g}(F)\right) & =\Sigma_{t}\left(F-k_{g}(F)\right)+\Delta_{t}\left(F-k_{g}(F)\right) \\
& =\Sigma_{t}\left(F-k_{g}(F)\right) \quad \text { from }(1.2 .82)
\end{array}
$$

Thus :

$$
W\left(M_{t}\left(F-k_{g}(F)\right) \mid \mathcal{F}_{g^{(t)}}\right)=W\left(\Sigma_{t}\left(F-k_{g}(F)\right) \mid \mathcal{F}_{g^{(t)}}\right)=0 \quad \text { from (1.2.78) }
$$

This ends the proof of Theorem 1.2.11.

Proof of Theorem 1.2.12.

For this purpose, we need the following result, due to Azéma and Yor (see [AY]) : a $\left(\left(\mathcal{F}_{t}, t \geq 0\right), W\right)$ martingale $\left(M_{t}, t \geq 0\right)$ vanishes on the zeros of $\left(X_{u}, u \geq 0\right)$ if and only if for every $t \geq 0$ :

$$
W\left(M_{t} \mid \mathcal{F}_{g^{(t)}}\right)=0 .
$$

Suppose $k_{g}(F)=0$

From (1.2.83), we have : $W\left(M_{t} \mid \mathcal{F}_{g^{(t)}}\right)=W\left(k_{g}(F) \mid \mathcal{F}_{g^{(t)}}\right)=0$. Thus, from (1.2.90), $\left(M_{t}(F), t \geq 0\right)$ vanishes on the zeros of $\left(X_{u}, u \geq 0\right)$.

Conversely, suppose that $\left(M_{t}(F), t \geq 0\right)$ vanishes on the zeros of $\left(X_{u} \geq 0\right)$. Then we have from (1.2.90) and (1.2.83), for every $s$ and $t, s \leq t$ and $\Gamma_{s} \in b\left(\mathcal{F}_{s}\right)$, since $\Gamma_{s} 1_{s \leq g^{(t)}}$ is a $\mathcal{F}_{g^{(t)}}$ measurable r.v. :

$$
\begin{aligned}
0 & =W\left(\Gamma_{s} 1_{s<g^{(t)}} M_{t}\left(k_{g}(F)\right)\right) \\
& =\mathbf{W}\left(\Gamma_{s} 1_{s<g^{(t)}} k_{g}(F) \underset{t \rightarrow \infty}{\longrightarrow} \mathbf{W}\left(\Gamma_{s} 1_{s \leq g} k_{g}(F)\right)\right.
\end{aligned}
$$

since $g^{(t)} \underset{t \rightarrow \infty}{\longrightarrow} g \quad \mathbf{W}$ a.s.

Thus :

$$
\mathbf{W}\left(\Gamma_{s} 1_{s \leq g} k_{g}(F)\right)=0
$$


We deduce from the monotone class Theorem that, for every bounded $\mathcal{F}_{g}$ measurable r.v. $\Phi$ :

$$
\mathbf{W}\left(\Phi k_{g}(F)\right)=0 .
$$

i.e. $k_{g}(F)=0$ since $k_{g}(F)$ is $\mathcal{F}_{g^{-}}$-measurable.

We end this subsection with some examples of decomposition (1.2.71).

Example 8. Let $F:=\exp \left(-\frac{\lambda}{2} L_{\infty}\right)$. We have shown (Example 2) that :

$$
M_{t}(F)=\left(\frac{2}{\lambda}+\left|X_{t}\right|\right) e^{-\frac{\lambda}{2} L_{t}}
$$

We then have :

$$
\begin{aligned}
& M_{t}(F)=\Sigma_{t}(F)+\Delta_{t}(F) \quad \text { with } \\
& \Sigma_{t}(F)=\left|X_{t}\right| e^{-\frac{\lambda}{2} L_{t}}, \Delta_{t}(F)=\frac{2}{\lambda} e^{-\frac{\lambda}{2} L_{t}}
\end{aligned}
$$

Indeed, from (1.2.72) :

$$
\begin{aligned}
\mathbf{W}\left(\Gamma_{t} 1_{g \leq t} e^{-\frac{\lambda}{2} L_{t}}\right) & =W\left(\Gamma_{t} \Sigma_{t}\left(e^{-\frac{\lambda}{2} L_{\infty}}\right)\right) \\
& =\mathbf{W}\left(\Gamma_{t} 1_{g \leq t} e^{-\frac{\lambda}{2} L_{t}}\right) \quad\left(\text { since } L_{\infty}=L_{t} \text { on the set }(g \leq t)\right) \\
& =W\left(\Gamma_{t}\left|X_{t}\right| e^{-\frac{\lambda}{2} L_{t}}\right)
\end{aligned}
$$

from point 2 i) of Theorem 1.1.6.

Thus :

$$
\Sigma_{t}\left(e^{-\frac{\lambda}{2} L_{\infty}}\right)=\left|X_{t}\right| e^{-\frac{\lambda}{2} L_{t}}
$$

Example 9. This example generalises Example 8. Let $q \in \mathcal{I}$ and $F:=\exp \left(-\frac{1}{2} A_{\infty}^{(q)}\right)$. We know (see Example 1) that :

$$
M_{t}\left(e^{-\frac{1}{2} A_{\infty}^{(q)}}\right)=\varphi_{q}\left(X_{t}\right) \exp \left(-\frac{1}{2} A_{t}^{(q)}\right)
$$

Then :

$$
\Sigma_{t}\left(e^{-\frac{1}{2} A_{\infty}^{(q)}}\right)=\psi_{q}\left(X_{t}\right) e^{-\frac{1}{2} A_{t}^{(q)}}, \Delta_{t}\left(e^{-\frac{1}{2} A_{\infty}^{(q)}}\right)=\left(\varphi_{q}-\psi_{q}\right)\left(X_{t}\right) e^{-\frac{1}{2} A_{t}^{(q)}}
$$

with $\psi_{q}$ solution of :

$$
\begin{aligned}
& \psi^{\prime \prime}=q \psi \quad \text { on } \mathbb{R} \\
& \psi(x) \underset{|x| \rightarrow \infty}{\sim}|x|, \quad \psi(0)=0
\end{aligned}
$$

Proof of (1.2.95). We have :

$$
\begin{aligned}
\mathbf{W}\left(\Gamma_{t} 1_{g \leq t} e^{\left.-\frac{1}{2} A_{\infty}^{(q)}\right)}=\right. & \varphi_{q}(0) W_{\infty}^{(q)}\left(\Gamma_{t} 1_{g \leq t}\right) \quad(\text { from }(1.1 .16)) \\
& (\text { with the notation of Theorems 1.1.2 and 1.1.1) } \\
= & \varphi_{q}(0) W_{\infty}^{(q)}\left(\Gamma_{t} W_{\infty}^{(q)}\left(1_{g \leq t} \mid \mathcal{F}_{t}\right)\right) \\
= & \varphi_{q}(0) W_{\infty}^{(q)}\left(\Gamma_{t} W_{\infty, X_{t}}^{(q)}\left(T_{0}=\infty\right)\right)
\end{aligned}
$$


But, by using the scale function $\gamma_{q}$ of the Markov process $\left(X_{t}, t \geq 0\right)$ under $W_{\infty}^{(q)}$, we have, with $\gamma_{q}$ given by (1.1.14) :

$$
\begin{aligned}
& W_{\infty, x}^{(q)}\left(T_{0}=\infty\right)=\frac{\gamma_{q}(x)-\gamma_{q}(0)}{\gamma_{q}(\infty)-\gamma_{q}(0)} \quad \text { if } x>0 \\
& =\frac{\gamma_{q}(0)-\gamma_{q}(x)}{\gamma_{q}(0)-\gamma_{q}(-\infty)} \quad \text { if } x<0 \\
& :=\lambda_{q}(x)
\end{aligned}
$$

Hence, by definition of $\Sigma_{t}\left(e^{-\frac{1}{2} A_{\infty}^{(q)}}\right)$ :

$$
\begin{aligned}
W\left(\Gamma_{t} \Sigma_{t}\left(e^{-\frac{1}{2} A_{\infty}^{(q)}}\right)\right) & =\varphi_{q}(0) W_{\infty}^{(q)}\left(\Gamma_{t} \lambda_{q}\left(X_{t}\right)\right) \\
& \left.=W\left(\Gamma_{t} \varphi_{q}\left(X_{t}\right) \lambda_{q}\left(X_{t}\right) e^{-\frac{1}{2} A_{t}^{(q)}}\right)\right)
\end{aligned}
$$

Thus :

$$
\Sigma_{t}\left(e^{-\frac{1}{2} A_{\infty}^{(q)}}\right)=\psi_{q}\left(X_{t}\right) e^{\frac{1}{2} A_{t}^{(q)}}
$$

with

$$
\psi_{q}(x):=\lambda_{q}(x) \varphi_{q}(x)
$$

It is clear, from (1.2.100), (1.2.98) and since $\varphi_{q}(x) \underset{|x| \rightarrow \infty}{\sim}|x|$ that :

$$
\psi_{q}(x) \underset{|x| \rightarrow \infty}{\sim}|x| \text { and } \quad \psi_{q}(0)=0 .
$$

On the other hand, the relation $\psi_{q}^{\prime \prime}=q \psi_{q}$ on $\mathbb{R}$ is the consequence of direct calculation by using the explicit form of $\gamma_{q}$ given by (1.1.14) (see Lemma 1.3.3 below for such a computation). We deduce from (1.2.95) and from Itô-Tanaka :

$$
\begin{aligned}
\Sigma_{t}\left(e^{-\frac{1}{2} A_{\infty}^{(q)}}\right)= & \int_{0}^{t} \psi_{q}^{\prime}\left(X_{s}\right) e^{-\frac{1}{2} A_{s}^{(q)}} d X_{s}+\int_{0}^{t}\left(\psi_{q}^{\prime}\left(0_{+}\right)-\psi_{q}^{\prime}\left(0_{-}\right)\right) e^{-\frac{1}{2} A_{s}^{(q)}} d L_{s} \\
\text { i.e. } \quad & M_{t}^{\Sigma(F)}=\int_{0}^{t} \psi_{q}^{\prime}\left(X_{s}\right) e^{-\frac{1}{2} A_{s}^{(q)}} d X_{s} \\
& k_{s}\left(e^{-\frac{1}{2} A_{\infty}^{(q)}}\right)=\left(\psi_{q}^{\prime}\left(0_{+}\right)-\psi_{q}^{\prime}\left(0_{-}\right)\right) e^{-\frac{1}{2} A_{s}^{(q)}} .
\end{aligned}
$$

Example 10. Let $\psi: \mathbb{R}_{+} \rightarrow \mathbb{R}_{+}$Borel and integrable with $\psi(\infty)=0$ and $F:=\psi\left(S_{\infty}\right)$. We know (see Example 9) that :

$$
M_{t}\left(\psi\left(S_{\infty}\right)\right)=\psi\left(S_{t}\right)\left(S_{t}-X_{t}\right)+\int_{S_{t}}^{\infty} \psi(y) d y
$$

We have :

$$
\Sigma_{t}\left(\psi\left(S_{\infty}\right)\right)=\psi\left(S_{t}\right) X_{t}^{-}, \quad \Delta_{t}\left(\psi\left(S_{\infty}\right)\right)=\psi\left(S_{t}\right)\left(S_{t}-X_{t}^{+}\right)+\int_{S_{t}}^{\infty} \psi(y) d y
$$

Indeed :

$$
\mathbf{W}\left(\Gamma_{t} 1_{g \leq t} \psi\left(S_{\infty}\right)=\mathbf{W}^{-}\left(\Gamma_{t} 1_{g \leq t} \psi\left(S_{g}\right)\right.\right.
$$


(since $\psi(\infty)=\infty, S_{\infty}=\infty$ on $\Gamma_{+}, S_{\infty}=S_{g}$ on $\left.\Gamma_{-}\right)$.

$$
\begin{aligned}
& =\mathbf{W}^{-}\left(\Gamma_{t} 1_{g \leq t} \psi\left(S_{g^{(t)}}\right)\right) \quad\left(\text { since } g^{(t)}=g \text { on }(g \leq t)\right) \\
& =W\left(\Gamma_{t} \psi\left(S_{g^{(t)}}\right) X_{t}^{-}\right) \quad \text { (from Theorem 1.1.5) } \\
& =W\left(\Gamma_{t} \psi\left(S_{t}\right) X_{t}^{-}\right) \quad\left(\text { since } S_{g^{(t)}}=S_{t} \text { if } X_{t}<0\right)
\end{aligned}
$$

Thus $\quad \Sigma_{t}\left(\psi\left(S_{\infty}\right)\right)=\psi\left(S_{t}\right) X_{t}^{-}$

Example 11. In some sense, the present example stands midway between Examples 9 and 10. Let $q: \mathbb{R} \rightarrow \mathbb{R}_{+}$such that $q(x)=0$ if $x<0, q(x)>0$ if $x>0, \varliminf_{x \rightarrow \infty} q(x)>0$. We have shown, in $[\mathrm{RY}, \mathrm{IX}]$ (see also [RY, M]) the existence for every $x \in \mathbb{R}$ of a $\sigma$-finite measure $\nu_{x}^{(q)}$, on $\mathbb{R}_{+}$such that :

$$
M_{t}\left(h\left(A_{\infty}^{(q)}\right)\right)=\int_{\mathbb{R}_{+}} h\left(A_{t}^{(q)}+y\right) \nu_{X_{t}}^{(q)}(d y)
$$

for $h: \mathbb{R}_{+} \rightarrow \mathbb{R}_{+}$sub exponential at infinity.

We then have :

$$
\begin{aligned}
\Sigma_{t}\left(h\left(A_{\infty}^{(q)}\right)\right) & =h\left(A_{t}^{(q)}\right) \cdot X_{t}^{-} \\
\Delta_{t}\left(h\left(A_{\infty}^{(q)}\right)\right) & =\int_{\mathbb{R}_{+}} h\left(A_{t}^{(q)}+y\right)\left(\nu_{X_{t}}^{(q)}(d y)-X_{t}^{-} \delta_{0}(d y)\right) \\
& =\int_{\mathbb{R}_{+}} h\left(A_{t}^{(q)}+y\right) \nu_{X_{t}}^{(q), a}(d y)
\end{aligned}
$$

where $\nu_{X_{t}}^{(q), a}$ denotes the absolute continuous part of $\nu_{X_{t}}^{(q)}$. Relation (1.2.104) is obtained from the same arguments as those used for relation (1.2.102) by noting that $1_{X_{t} \leq 0} d A_{t}^{(q)}=0$ and (1.2.105) results from :

$$
\begin{aligned}
& \text { if } x<0, \nu_{x}^{(q)}(d y)=\nu_{x}^{(q), a}(d y)+x^{-} \delta_{0}(d y) \\
& \text { if } x>0, \nu_{x}^{(q)}(d y)=\nu_{x}^{(q), a}(d y)
\end{aligned}
$$

Example 12. Let $q: \mathbb{R} \rightarrow \mathbb{R}_{+}$such that :

$$
\int_{-\infty}^{0}(1+|x|) q(x) d x<\infty ; \underline{\lim _{x \rightarrow \infty}} x^{2 \alpha} q(x)>0 \quad \text { for some } \alpha<1
$$

and $A_{t}^{(q)}:=\int_{0}^{t} q\left(X_{s}\right) d s$. Let $\varphi_{q}$ the solution of $\varphi^{\prime \prime}=q \varphi, \quad \varphi^{\prime}(-\infty)=-1, \quad \varphi(+\infty)=0$. Then, we have :

$$
\begin{aligned}
& \quad M_{t}\left(\exp -\frac{1}{2} A_{\infty}^{(q)}\right)=\varphi_{q}\left(X_{t}\right) \exp \left(\exp -\frac{1}{2} A_{t}^{(q)}\right) \\
& \text { and } \quad e^{-\frac{1}{2} A_{\infty}^{(q)}} \cdot \mathbf{W}^{-}=\mathbf{W}\left(e^{-\frac{1}{2} A_{\infty}^{(q)}}\right) \cdot W_{\infty}^{(q)}
\end{aligned}
$$

where the probability $W_{\infty}^{(q)}$ is characterised by

$$
\left.W_{\infty}^{(q)}\right|_{\mathcal{F}_{t}}=\left.\frac{\varphi_{q}\left(X_{t}\right)}{\varphi_{q}(0)} \exp \left(-\frac{1}{2} A_{t}^{(q)}\right) \cdot W\right|_{\mathcal{F}_{t}}
$$


(see [RVY, I], the one-sided case, p. 209). We then have :

$$
\Sigma_{t}\left(e^{-\frac{1}{2} A_{\infty}^{(q)}}\right)=\psi_{q}\left(X_{t}\right) e^{-\frac{1}{2} A_{t}^{(q)}}
$$

with

$$
\begin{aligned}
& \psi_{q}(x)=0 \quad \text { if } x \geq 0 \\
& \psi_{q}(x) \underset{x \rightarrow-\infty}{\sim}|x| \quad \text { and } \quad \psi_{q}^{\prime \prime}=q \psi_{q} \text { on } \mathbb{R}_{-}
\end{aligned}
$$

Hence

$$
\Delta_{t}\left(e^{-\frac{1}{2} A_{\infty}^{(q)}}\right)= \begin{cases}M_{t}\left(e^{-\frac{1}{2} A_{\infty}^{(q)}}\right) & \text { if } X_{t} \geq 0 \\ \left(\varphi_{q}-\psi_{q}\right)\left(X_{t}\right) e^{-\frac{1}{2} A_{t}^{(q)}} & \text { if } X_{t} \leq 0\end{cases}
$$

(1.2.109) is obtained by following the same arguments as those in example 9 . What changes is that, under the probability $W_{x, \infty}^{(q)}$, we have $X_{t} \rightarrow-\infty$ a.s., for every $x$ (see Theorem 5.1 in $[R V Y, I])$.

Example 13. Let $\psi: \mathbb{R}_{+} \rightarrow \mathbb{R}_{+}$Borel and integrable, such that $\int_{0}^{\infty} \psi(y) d y=1$. Then we have, from (1.2.24)

$$
M_{t}\left(\psi\left(S_{g}\right)\right)=\frac{1}{2} \psi\left(S_{g^{(t)}}\right)\left|X_{t}\right|+\psi\left(S_{t}\right)\left(S_{t}-X_{t}^{+}\right)+\int_{S_{t}}^{\infty} \psi(y) d y
$$

(see Example 4, (1.2.24) and (1.2.25)) ;

$$
\mathbf{W}^{-}\left(\psi\left(S_{g}\right)\right)=\mathbf{W}\left(\psi\left(S_{\infty}\right)\right)=\int_{0}^{\infty} \psi(l) d l
$$

On the other hand, we have :

$$
\begin{aligned}
\mathbf{W}\left(\Gamma_{t} 1_{g \leq t} \psi\left(S_{g}\right)\right) & =W\left(\Gamma_{t} \Sigma_{t}\left(\psi\left(S_{g}\right)\right)\right) \\
& =\mathbf{W}\left(\Gamma_{t} 1_{g \leq t} \psi\left(S_{g^{(t)}}\right)\right) \quad\left(\text { since } g=g^{(t)} \text { on }(g \leq t)\right) \\
& =W\left(\Gamma_{t} \psi\left(S_{g^{(t)}}\right)\left|X_{t}\right|\right) \quad(\text { from point } 2 i) \text { of Theorem 1.1.6.) }
\end{aligned}
$$

Hence :

$$
\Sigma_{t}\left(\psi\left(S_{g}\right)\right)=\psi\left(S_{g^{(t)}}\right)\left|X_{t}\right|
$$

and, from (1.2.110) :

$$
\Delta_{t}\left(\psi\left(S_{g}\right)\right)=\psi\left(S_{t}\right)\left(S_{t}-X_{t}^{+}\right)+\int_{S_{t}}^{\infty} \psi(y) d y
$$

\subsubsection{A penalisation Theorem.}

In section 1 of this chapter, we constructed the measure $\mathbf{W}$ from the penalisation results, and more particularly from Feynman-Kac type penalisations. We shall now operate in a reverse order : starting from the existence and the properties of the measure $\mathbf{W}$ which we just established, we shall obtain a penalisation result. 
Unfortunately, we are not able to establish a "general result" of penalisation : for technical reasons, we shall need to limit ourselves to a particular class of penalisation processes $\left(F_{t}, t \geq 0\right)$. We begin by describing this class.

Let $\left(F_{t}, t \geq 0\right)$ denote an adapted, positive process. We shall say that this process belongs to the class $\mathcal{C}$ if :

i) $\left(F_{t}, t \geq 0\right)$ is a decreasing process, i.e. : if $s \leq t$ :

$$
0 \leq F_{t} \leq F_{s} \quad W \text { a.s. }
$$

In particular, since $0 \leq F_{t} \leq F_{0}$ and since $F_{0}$ is a.s. constant, this process is bounded by a constant $C=F_{0}$.

ii) There exists $a \geq 0$ such that for every $t \geq \sigma_{a}$, with :

$$
\sigma_{a}:=\sup \left\{t \geq 0 ; X_{t} \in[-a, a]\right\}
$$

we have :

$$
F_{t}=F_{\sigma_{a}}=F_{\infty}
$$

and

$$
\mathbf{W}\left(F_{\infty}\right)=\mathbf{W}\left(F_{\sigma_{a}}\right)<\infty
$$

One of the advantages of this class $\mathcal{C}$ is that it contains a large number of processes $\left(F_{t}, t \geq 0\right)$ for which we have already obtained a penalisation result. More precisely, let $\varphi: \mathbb{R}^{n} \rightarrow \mathbb{R}_{+}$ Borel. Then :

$$
F_{t}:=\varphi\left(L_{t}^{a_{n}}, \cdots L_{t}^{a_{r}}, A_{t}^{\left(q_{1}\right)}, \cdots A_{t}^{\left(q_{s}\right)}, D_{t}^{\left[\alpha_{1}, \beta_{1}\right]}, \cdots D_{t}^{\left[\alpha_{u}, \beta_{u}\right]}, S_{g^{(t)}},-I_{g^{(t)}}\right)
$$

(see Examples 1 to 9 for these notations) belongs to the class $\mathcal{C}$ (if (1.2.115) is satisfied) as soon as $q_{1}, \cdots q_{s}$ are elements of $\mathcal{I}$ with compact support (if we choose a large enough) and that $\varphi$ is a function which is decreasing with respect to each of its arguments. We may add $S_{t}$ and $\left(-I_{t}\right)$ to the list of the arguments of $\varphi$, if $\varphi$ has compact support in these arguments. Here is the first step towards a penalisation result.

Proposition 1.2.13. Let $\left(F_{t}, t \geq 0\right)$ be a process which belongs to $\mathcal{C}$. Then :

$$
\sqrt{\frac{\pi t}{2}} W\left(F_{t}\right) \underset{t \rightarrow \infty}{\longrightarrow} \mathbf{W}\left(F_{\infty}\right)
$$

Proof of Proposition 1.2.13.

We write $F_{t}$ in the form :

$$
\begin{aligned}
F_{t}= & F_{t} \frac{\left(\left|X_{t}\right|-a\right)_{+}}{1+\left|X_{t}\right|}+F_{t} \frac{1+\left|X_{t}\right|-\left(\left|X_{t}\right|-a\right)_{+}}{\left(1+\left|X_{t}\right|\right)^{2}}\left(\left|X_{t}\right|-a\right)_{+} \\
& +F_{t} \frac{\left[\left(1+\left|X_{t}\right|\right)-\left(\left|X_{t}\right|-a\right)_{+}\right]^{2}}{\left(1+\left[X_{t} \mid\right)^{2}\right.}:=F_{t}^{(1)}+F_{t}^{(2)}+F_{t}^{(3)}
\end{aligned}
$$

and we study each term of this decomposition of $F_{t}$.

i) Study of $W\left(F_{t}^{(1)}\right)$. 
For $\lambda>0$, we have :

$$
\begin{aligned}
\int_{0}^{\infty} & e^{-\lambda t} W\left(F_{t}^{(1)}\right) d t=\int_{0}^{\infty} e^{-\lambda t} W\left(F_{t} \frac{\left.\left(\left|X_{t}\right|-a\right)_{+}\right)}{1+\left|X_{t}\right|}\right) d t \\
& =\int_{0}^{\infty} e^{-\lambda t} \mathbf{W}\left(\frac{F_{t}}{1+\left|X_{t}\right|} 1_{\sigma_{a} \leq t}\right)
\end{aligned}
$$

(by Theorem 1.1.8, relation $(1.1 .48)$ )

$$
\begin{aligned}
& =\int_{0}^{\infty} e^{-\lambda t} \mathbf{W}\left(F_{\sigma_{a}} \frac{1_{\sigma_{a} \leq t}}{1+\left|X_{t}\right|}\right) d t \quad(\text { from (1.2.114)) } \\
& =\mathbf{W}\left(F_{\sigma_{a}} e^{-\lambda \sigma_{a}} \int_{0}^{\infty} e^{-\lambda u} \frac{d u}{1+\left|X_{\sigma_{a}+u}\right|}\right)
\end{aligned}
$$

(after the change of variable $t=\sigma_{a}+u$ )

$$
=\mathbf{W}\left(F_{\infty} e^{-\lambda \sigma_{a}}\right) E_{0}^{(3)}\left(\int_{0}^{\infty} e^{-\lambda u} \frac{d u}{1+a+R_{u}}\right)
$$

from point 2 of Theorem 1.1.8, where in (1.2.118) $\left(R_{u}, u \geq 0\right)$ denotes a Bessel process of dimension 3 started at 0 . But

$$
E_{0}^{(3)}\left[\frac{1}{1+a+R_{u}}\right] \underset{u \rightarrow \infty}{\sim} \sqrt{\frac{2}{\pi u}}
$$

and is a decreasing function of $u$. By the (easy part of the) Tauberian Theorem (see [Fel]) :

$$
\int_{0}^{\infty} e^{-\lambda t} W\left(F_{t}^{(1)}\right) d t \underset{\lambda \rightarrow 0}{\sim} \mathbf{W}\left(F_{\infty}\right) \sqrt{\frac{2}{\lambda}}
$$

ii) Study of $W\left(F_{t}^{(2)}\right)$.

For $\lambda>0$, we have :

$$
\int_{0}^{\infty} e^{-\lambda t} W\left(F_{t}^{(2)}\right) d t \leq(1+a) \int_{0}^{\infty} e^{-\lambda t} W\left(F_{t} \frac{\left(\left|X_{t}\right|-a\right)_{+}}{\left.1+\left|X_{t}\right|\right)^{2}}\right) d t
$$

(from (1.2.117) and since : $\left.0 \leq 1+\left|X_{t}\right|-\left(\left|X_{t}\right|-a\right)_{+} \leq 1+a\right)$

$$
=(1+a) \mathbf{W}\left(F_{\infty} e^{-\lambda \sigma_{a}}\right) E_{0}^{(3)}\left(\int_{0}^{\infty} e^{-\lambda u} \frac{d u}{\left(1+a+R_{u}\right)^{2}}\right)
$$

(by using the same argument as in point $i$ ))

$$
\begin{aligned}
& \leq(1+a) \mathbf{W}\left(F_{\infty}\right) \int_{0}^{\infty} e^{-\lambda u} E_{0}^{(3)}\left(\frac{1}{\left(1+a+R_{u}\right)^{\frac{3}{2}}}\right) d u \\
& \leq(1+a) \mathbf{W}\left(F_{\infty}\right) o\left(\frac{1}{\lambda^{\frac{1}{4}}}\right)=o\left(\frac{1}{\sqrt{\lambda}}\right) \quad(\lambda \rightarrow 0)
\end{aligned}
$$

iii) Study of $W\left(F_{t}^{(3)}\right)$.

$$
W\left(F_{t}^{(3)}\right) \leq(1+a)^{2} C W\left(\frac{1}{1+\left|X_{t}\right|^{2}}\right)
$$


from (1.2.117). Hypothesis i) : $0 \leq F_{t} \leq C$ imply :

$$
\sqrt{\frac{\pi t}{2}} W\left(F_{t}^{(3)}\right) \underset{t \rightarrow \infty}{\longrightarrow} 0
$$

Thus :

$$
\int_{0}^{\infty} e^{-\lambda t} W\left(F_{t}^{(3)}\right) d t=o\left(\frac{1}{\sqrt{\lambda}}\right) \quad(\lambda \rightarrow 0)
$$

Gathering (1.2.119), (1.2.120) and (1.2.122) we obtain :

$$
\int_{0}^{\infty} e^{-\lambda t} W\left(F_{t}\right) d t \underset{\lambda \rightarrow 0}{\sim} \sqrt{\frac{2}{\lambda}} \mathbf{W}\left(F_{\infty}\right)
$$

$W\left(F_{t}\right)$ being by hypothesis a decreasing function in $t$, the Tauberian Theorem implies :

$$
\sqrt{\frac{\pi t}{2}} W\left(F_{t}\right) \underset{t \rightarrow \infty}{\longrightarrow} \mathbf{W}\left(F_{\infty}\right)
$$

This is precisely the statement of Proposition 1.2.13. We are now able to state the announced penalisation Theorem.

Theorem 1.2.14. (General penalisation Theorem)

Let $\left(F_{t}, t \geq 0\right)$ be a process belonging to $\mathcal{C}$. Then, for every $s \geq 0$ and $\Gamma_{s} \in b\left(\mathcal{F}_{s}\right)$ :

1) The limit, as $t \rightarrow \infty, \frac{W\left(\Gamma_{s} F_{t}\right)}{W\left(F_{t}\right)}$ exists

2) This limit equals :

$$
\lim _{t \rightarrow \infty} \frac{W\left(\Gamma_{s} F_{t}\right)}{W\left(F_{t}\right)}=\frac{W\left(\Gamma_{s} M_{s}\left(F_{\infty}\right)\right)}{\mathbf{W}\left(F_{\infty}\right)}:=W_{\infty}^{F}\left(\Gamma_{s}\right)
$$

The probability $W_{\infty}^{F}$, which is characterised by (1.2.125) satisfies :

$$
W_{\infty}^{F}=\frac{F_{\infty}}{\mathbf{W}\left(F_{\infty}\right)} \cdot \mathbf{W}
$$

Proof of Theorem 1.2.14.

i) We shall use the following notations : let $\omega_{s} \in \mathcal{C}([0, s] \rightarrow \mathbb{R})$ and $\left(F_{t}^{\left(\omega_{s}\right)}, t \geq 0\right)$ the functional defined by :

$$
F_{t}^{\left(\omega_{s}\right)}\left(X_{u}, u \geq 0\right):=F_{t+s}\left(\omega_{s} \circ\left(\omega_{s}(s)+X_{u}, u \geq 0\right)\right)
$$

With this notation, we have the following Lemma.

Lemma 1.2.15. If $\left(F_{t}, t \geq 0\right) \in \mathcal{C}$, then, for every $\omega_{s} \in \mathcal{C}([0, s] \rightarrow \mathbb{R})\left(F_{t}^{\left(\omega_{s}\right)}, t \geq 0\right) \in \mathcal{C}$.

Proof of Lemma 1.2.15.

i) It is clear that $\left(F_{t}^{\left(\omega_{s}\right)}, t \geq 0\right)$ is a decreasing function of $t$ and that, from (1.2.127) and (1.2.114) we have, for $t \geq \sigma_{\left|\omega_{s}(s)\right|+a}$ :

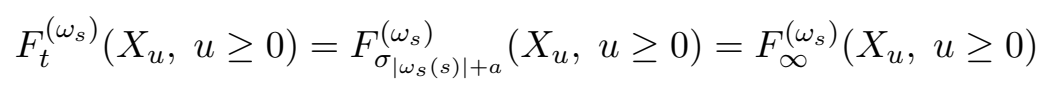


ii) We need to prove that $\mathbf{W}\left(F_{\infty}^{\left(\omega_{s}\right)}\right)<\infty$. We note that :

$$
\begin{aligned}
\mathbf{W}\left(F_{\infty}^{\left(\omega_{s}\right)}\right) & =\mathbf{W}\left(F_{\infty}\left(\omega_{s} \circ\left(\omega_{s}(s)+X_{u}, u \geq 0\right)\right)\right. \\
& =M_{s}\left(F_{\infty}\right)\left(\omega_{s}\right) \quad(\operatorname{from}(1.2 .3))
\end{aligned}
$$

Hence :

$$
W\left(\mathbf{W}\left(F_{\infty}^{\left(\omega_{s}\right)}\right)\right)=W\left(M_{s}\left(F_{\infty}\right)\right)=\mathbf{W}\left(F_{\infty}\right)<\infty \quad(\text { from }(1.2 .2))
$$

In particular :

$$
\mathbf{W}\left(F_{\infty}^{\left(\omega_{s}\right)}\right)<\infty \quad W \text { a.s. }
$$

This is Lemma 1.2.15.

ii) We may now end the proof of Theorem 1.2.14. We have, for $t \geq s$ :

$$
\begin{aligned}
\frac{W\left(F_{t} \mid \mathcal{F}_{s}\right)}{W\left(F_{t}\right)} & =\frac{\widehat{W}\left(F_{t-s}^{\left(\omega_{s}\right)}\right)}{W\left(F_{t}\right)} \quad \text { (from the Markov property) } \\
& =\frac{\sqrt{\frac{\pi t}{2}} W\left(F_{t-s}^{\left(\omega_{s}\right)}\right)}{\sqrt{\frac{\pi t}{2}} W\left(F_{t}\right)} \underset{t \rightarrow \infty}{\longrightarrow} \frac{\mathbf{W}\left(F_{\infty}^{\left(\omega_{s}\right)}\right)}{\mathbf{W}\left(F_{\infty}\right)} \quad \text { a.s. }
\end{aligned}
$$

(from Proposition 1.2.13 applied to $\left(F_{t}, t \geq 0\right)$ and to $\left(F_{t}^{\left(\omega_{s}\right)}, t \geq 0\right)$ due to Lemma 1.2.15.)

$$
=\frac{M_{s}\left(F_{\infty}\right)}{\mathbf{W}\left(F_{\infty}\right)}
$$

(from point 2 of Theorem 1.2.1.)

To show Theorem 1.2.14, it now suffices to see that the convergence is (1.2.128) also holds in $L^{1}\left(\mathcal{F}_{\infty}, W\right)$. However, from Scheffé Lemma (see [Mey], T. 21) this will be implied by the equality : $\left(\frac{M_{s}\left(F_{\infty}\right)}{\mathbf{W}\left(F_{\infty}\right)}\right)=1$ for every $s \geq 0$, which follows immediatly from Theorem 1.2.1.

\section{Remark 1.2.16.}

Let $\varphi: \mathbb{R}_{+} \rightarrow \mathbb{R}_{+}$Borel such that : $\int_{0}^{\infty} \varphi(x) x^{2} d x<\infty$ and let :

$$
\begin{array}{ll}
F_{t}^{(1)}:=\varphi\left(S_{t}\right) 1_{\left(X_{t}>0\right)} & (t \geq 0) \\
F_{t}^{(2)}:=\varphi\left(S_{d_{t}}\right) 1_{\left(X_{t}>0\right)} & (t \geq 0)
\end{array}
$$

It is shown in [RY, VIII] that :

i) $\begin{aligned} E\left(F_{t}^{(1)}\right) \underset{t \rightarrow \infty}{\sim} \frac{3}{2} \sqrt{\frac{2}{\pi t^{3}}} \int_{0}^{\infty} \varphi(x) x^{2} d x \\ E\left(F_{t}^{(2)}\right) \underset{t \rightarrow \infty}{\sim} \sqrt{\frac{2}{\pi t^{3}}} \int_{0}^{\infty} \varphi(x) x^{2} d x\end{aligned}$

ii) for every $s \geq 0$ and $\Gamma_{s} \in b\left(\mathcal{F}_{s}\right)$

$$
\frac{E\left[\Gamma_{s} F_{t}^{(i)}\right]}{E\left(F_{t}^{(i)}\right)} \underset{t \rightarrow \infty}{\longrightarrow} E\left(\Gamma_{s} M_{s}^{\psi}\right) \quad(i=1,2)
$$


where the martingale $\left(M_{s}^{\psi}, s \geq 0\right)$ is defined by :

$$
\begin{aligned}
M_{s}^{\psi} & =\psi\left(S_{s}\right)\left(S_{s}-X_{s}\right)+\int_{S_{s}}^{\infty} \psi(y) d y \\
\text { and } \quad \psi(x) & :=\varphi(x) x^{2}+2 \int_{x}^{\infty} \varphi(y) y d y \quad(x \geq 0)
\end{aligned}
$$

We now inspect Theorem 1.2.14 in the light of this results. If we assume that $\lim _{y \rightarrow+\infty} \varphi(y)=0$, we obtain :

$$
\lim _{y \rightarrow \infty} F_{t}^{(i)}=0 \quad \mathbf{W} \quad \text { a.s. }
$$

and, from (1.2.129) and (1.2.130).

$$
\lim _{t \rightarrow \infty} \sqrt{t} E\left[F_{t}^{(i)}\right]=0 \quad(i=1,2)
$$

Thus, we are working here in a degenerate case of Theorem 1.2.14 and of Proposition 1.2.13, i.e. : in a case where $F_{\infty} \equiv 0$. However, from (1.2.131), this situation is not so "degenerate", since it allows to obtain a non-trivial penalisation.

Here is a variation on Proposition 1.2.13, which is easier to prove :

Theorem 1.2.17.

Let $\left(F_{t}, t \geq 0\right)$ be a functional belonging to $\mathcal{C}$. Then :

$$
W\left(F_{t} \cdot\left|X_{t}\right|\right) \underset{t \rightarrow \infty}{\longrightarrow} \mathbf{W}\left(F_{\infty}\right)
$$

Proof of (1.2.132).

We write

$$
\begin{aligned}
W\left(F_{t} \cdot\left|X_{t}\right|\right) & =W\left(F_{t}\left(\left|X_{t}\right|-a\right)_{+}\right)+W\left(F_{t}\left(\left|X_{t}\right|-\left(\left|X_{t}\right|-a\right)_{+}\right)\right. \\
& :=\left(1_{t}\right)+\left(2_{t}\right)
\end{aligned}
$$

and we study successively $\left(1_{t}\right)$ and $\left(2_{t}\right)$.

$$
\begin{aligned}
\cdot\left(1_{t}\right)= & W\left(F_{t}\left(\left|X_{t}\right|-a\right)_{+}\right)=\mathbf{W}\left(F_{t} 1_{\sigma_{a} \leq t}\right) \quad \text { (from Theorem 1.1.8) } \\
= & \mathbf{W}\left(F_{\infty} 1_{\sigma_{a} \leq t}\right) \quad \text { from }(1.2 .114) \\
& \underset{t \rightarrow \infty}{\longrightarrow} \mathbf{W}\left(F_{\infty}\right) \quad(\text { by the monotone convergence Theorem) } \\
& \left(\text { since } F_{\infty} \in L_{+}^{1}\left(\mathcal{F}_{\infty}, \mathbf{W}\right)\right) \\
\cdot\left(2_{t}\right)= & W\left(F_{t}\left(\left|X_{t}\right|-\left(\left|X_{t}\right|-a\right)_{+}\right) \leq a W\left(F_{t}\right)\right.
\end{aligned}
$$


We now write :

$$
\begin{aligned}
W\left(F_{t}\right)= & W\left(F_{t} \frac{1+\left|X_{t}\right|-\left(\left|X_{t}\right|-a\right)_{+}}{1+\left|X_{t}\right|}\right)+W\left(F_{t} \frac{\left(\left|X_{t}\right|-a\right)_{+}}{1+\left|X_{t}\right|}\right) \quad(1.2 .133) \\
= & \left(3_{t}\right)+\left(4_{t}\right) \quad \text { and we have } \\
\left(3_{t}\right)= & W\left(F_{t} \frac{1+\left|X_{t}\right|-\left(\left|X_{t}\right|-a\right)_{+}}{1+\left|X_{t}\right|}\right) \leq(1+a) W\left(\frac{F_{t}}{1+\left|X_{t}\right|}\right) \\
& \leq(1+a) C W\left(\frac{1}{1+\left|X_{t}\right|}\right) \underset{t \rightarrow \infty}{\longrightarrow} 0 \\
\text { since } & \left(F_{t}, t \geq 0\right) \text { is bounded } \\
\left(4_{t}\right)= & W\left(F_{t} \frac{\left(\left|X_{t}\right|-a\right)_{+}}{1+\left|X_{t}\right|}\right)=\mathbf{W}\left(\frac{F_{t}}{1+\left|X_{t}\right|} 1_{\sigma_{a} \leq t}\right) \quad \text { (from Th. 1.1.8.) } \\
= & \mathbf{W}\left(\frac{F_{\infty}}{1+\left|X_{t}\right|} 1_{\sigma_{a} \leq t}\right) \quad(1.2 .114) \\
\underset{t \rightarrow \infty}{\longrightarrow} & 0 \quad \text { since }\left|X_{t}\right| \underset{t \rightarrow \infty}{\longrightarrow}+\infty \quad \mathbf{W} \text { a.s. and we apply the dominated convergence }
\end{aligned}
$$

1.3 Invariant measures related to $\mathrm{W}_{x}$ and $\Lambda_{x}$.

We shall now show that the measure $\mathbf{W}$, and the measure $\boldsymbol{\Lambda}$ which we shall define very soon, are closely related to invariant measures of some Markov process taking values in certain functional spaces.

1.3.1 The process $\left(\mathcal{X}_{t}, t \geq 0\right)$.

As before, $\left(\Omega,\left(X_{t}, \mathcal{F}_{t}\right)_{t \geq 0}, \mathcal{F}_{\infty}, W_{x}(x \in \mathbb{R})\right)$ denotes the canonical realisation of Brownian motion. Let $\mathcal{X}_{0} \in \Omega=\mathcal{C}\left(\mathbb{R}_{+} \rightarrow \mathbb{R}\right)$. We define the process $\left(\mathcal{X}_{t}, t \geq 0\right)$ taking values on $\mathcal{C}\left(\mathbb{R}_{+} \rightarrow \mathbb{R}\right)$, and issued from $\mathcal{X}_{0}$, by :

$$
\mathcal{X}_{t}(u):= \begin{cases}\mathcal{X}_{0}(u-t) & \text { if } \quad u \geq t \\ \mathcal{X}_{0}(0)+X_{t-u} & \text { if } u \leq t\end{cases}
$$

It is easy enough to see that this process is Markov (we denote by $\left(P_{t}, t \geq 0\right)$ the semigroup associated with this Markov Process $\left.\left(\mathcal{X}_{t}, t \geq 0\right)\right)$ and that the measure :

$$
\widetilde{\mathbf{W}}:=\int_{\mathbb{R}} d x W_{x}
$$

is an invariant measure for this process. However, this process admits other invariant measures. More precisely :

Theorem 1.3.1. Let $a, b \geq 0$, with $a+b>0$, and:

$$
\mathbf{W}_{x}^{a, b}:=a \mathbf{W}_{x}^{+}+b \mathbf{W}_{x}^{-}
$$

Then :

$$
\widetilde{\mathbf{W}}^{a, b}:=\int_{\mathbb{R}} d x \mathbf{W}_{x}^{a, b}
$$


is an invariant measure for the process $\left(\mathcal{X}_{t}, t \geq 0\right)$. Recall that $\mathbf{W}_{x}^{+}$and $\mathbf{W}_{x}^{-}$are defined in (1.1.88) by :

$$
\mathbf{W}_{x}^{+}=1_{\Gamma_{+}} \cdot \mathbf{W}_{x}, \mathbf{W}_{x}^{-}=1_{\Gamma_{-}} \cdot \mathbf{W}_{x}
$$

Proof of Theorem 1.3.1.

By symmetry, it suffices to prove that the measure $\widetilde{\mathbf{W}}^{+}$defined by $\widetilde{\mathbf{W}}^{+}:=\int_{\mathbb{R}} d x \mathbf{W}_{x}^{+}$is invariant. For every measurable and positive functional $F: \Omega \rightarrow \mathbb{R}_{+}$, we have :

$$
\begin{array}{rl}
\int_{\mathbb{R}} & d x \int_{\Omega} \mathbf{W}_{x}^{+}(d \mathcal{X}) P_{t} F(\mathcal{X}) \\
& =\int_{\mathbb{R}} d x \int_{\Omega} \mathbf{W}_{x}^{+}(d \mathcal{X}) W\left(F\left(x+X_{t-u}, u \leq t ; \mathcal{X}(v-t), v \geq t\right)\right) \\
& =\int_{\mathbb{R}} d x \int_{\Omega} \mathbf{W}_{x}^{+}(d \mathcal{X}) W\left(F\left(x+X_{t}-X_{u}, u \leq t ; \mathcal{X}(v-t), v \geq t\right)\right)
\end{array}
$$

(since $\left(X_{t-u}, u \leq t\right)$ has the same law under $W$ than $\left(X_{t}-X_{u}, u \leq t\right)$ )

$$
=\int_{\mathbb{R}} d y W\left(\int_{\Omega} \mathbf{W}_{y-X_{t}}^{+}(d \mathcal{X}) F\left(y-X_{u}, u \leq t ; \mathcal{X}(v-t), v \geq t\right)\right)
$$

(from Fubini and after making the change of variable $x+X_{t}=y$ )

$$
=\int_{\mathbb{R}} d y W\left(\int_{\Omega} \mathbf{W}_{y-X_{t}}(d \mathcal{X}) F\left(y-X_{u}, u \leq t ; \mathcal{X}(v-t), v \leq t\right) 1_{\Gamma_{+}}(\mathcal{X})\right)
$$

(from the definition of $\mathbf{W}^{+}$)

$$
=\int_{\mathbb{R}} d y W\left(M_{t}\left(F 1_{\Gamma_{+}}\right)\left(y-X_{u}, u \leq t\right)\right)
$$

(from the definition (1.2.3) of the martingale $\left(M_{t}\left(F 1_{\Gamma_{+}}\right), t \geq 0\right)$ )

$$
=\int_{\mathbb{R}} d y W\left(M_{t}\left(F 1_{\Gamma_{+}}\right)\left(y+X_{u}, u \leq t\right)\right)
$$

(since $\left(X_{u}, u \leq t\right)$ and $\left(-X_{u}, u \leq t\right)$ have the same law under $W$ )

$$
\begin{aligned}
& =\int_{\mathbb{R}} d y W_{y}\left(M_{t}\left(F 1_{\Gamma_{+}}\right)\right) \\
& =\int_{\mathbb{R}} d y W_{y}\left(M_{0}\left(F 1_{\Gamma_{+}}\right)\right)
\end{aligned}
$$

(since $\left(M_{t}\left(1_{\Gamma_{+}}\right), t \geq 0\right)$ is a $\left(\left(\mathcal{F}_{t}, t \geq 0\right), W\right)$ martingale)

$$
=\int_{\mathbb{R}} d y \mathbf{W}_{y}\left(F 1_{\Gamma_{+}}\right)=\widetilde{W}^{+}(F)
$$

(since $W_{y}\left(M_{0}\left(F 1_{\Gamma_{+}}\right)\right)=\mathbf{W}_{y}\left(F 1_{\Gamma_{+}}\right)$from $(1.2 .2)$.

This is Theorem 1.3.1. 


\subsubsection{The measure $\Lambda_{x}$.}

Let $\widetilde{\Omega}=\mathcal{C}\left(\mathbb{R} \rightarrow \mathbb{R}_{+}\right)$and $\mathcal{L}: \Omega \rightarrow \widetilde{\Omega}$, the application "total local time" defined by :

$$
\mathcal{L}\left(X_{t}, t \geq 0\right)=\left(L_{\infty}^{y}, y \in \mathbb{R}\right) .
$$

We denote by $\boldsymbol{\Lambda}_{x}$ the image of $\mathbf{W}_{x}$ by $\mathcal{L}$. It is possible to give a very simple description of $\boldsymbol{\Lambda}_{x}$ (see $\left.[\mathrm{RY}, \mathrm{M}]\right)$. Here is this description :

. Let $u, \alpha, \beta \in \mathbb{R}_{+}$and $x \in \mathbb{R}$. We denote by $Q_{x, u}^{\alpha, \beta}$ the law of the process $\left(Y_{v}, v \in \mathbb{R}\right)$ defined as follows :

$$
\begin{aligned}
& Y_{x}=u \\
& \left(Y_{x+t}, t \geq 0\right) \text { is the square of an } \alpha \text {-dimensional Bessel process } \\
& \left(Y_{x-t}, t \geq 0\right) \text { is the square of a } \beta \text {-dimensional Bessel process, independent from } \\
& \left(Y_{x+t}, t \geq 0\right) .
\end{aligned}
$$

Then :

$$
\boldsymbol{\Lambda}_{x}=\frac{1}{2} \int_{0}^{\infty} d u\left(Q_{x, u}^{0,2}+Q_{x, u}^{2,0}\right)
$$

Sketch of the proof of (1.3.6).

By translation, it suffices to prove (1.3.6) for $x=0$. Then, we use (1.1.40) :

$$
\mathbf{W}=\int_{0}^{\infty} d v\left(W_{0}^{\tau_{v}} \circ P_{0}^{(3, \mathrm{sym})}\right)
$$

and the following facts :

- From the second Ray-Knight Theorem (see [RY], Chap. IX) for Brownian motion, the process $\left(L_{\tau_{l}}^{y}, y \geq 0\right)$ is a 0-dimensional squared Bessel process, starting from $l$.

- For a 3-dimensional Bessel process, starting from $0,\left(L_{\infty}^{y}, y \geq 0\right)$ is a 2-dimensional squared Bessel process, starting from 0. This constitutes the "third" Ray-Knight Theorem.

- If $\left(Z_{t}^{i}, t \geq 0\right), i=1,2$, are two squared Bessel processes with respective dimensions $d_{1}$ and $d_{2}$, starting respectively from $u_{1}$ and $u_{2}$, then $\left(Z_{t}^{(1)}+Z_{t}^{(2)}, t \geq 0\right)$ is a squared Bessel process with dimension $d_{1}+d_{2}$ starting from $u_{1}+u_{2}$. Other properties about the measure $\boldsymbol{\Lambda}_{x}$ may be found in ([RY, M], Chap. 2). It is easily deduced from (1.3.6) that the r.v. $L_{\infty}^{y}$, under $\mathbf{W}_{x}$, admits the "law" :

$$
\mathbf{W}_{x}\left(L_{\infty}^{y} \in d u\right)=|y-x| \delta_{0}(d u)+d u \quad(u \geq 0)
$$

(see also (1.1.45)).

1.3.3 Invariant measures for the process $\left(\left(X_{t}, L_{t}^{\bullet}\right), t \geq 0\right)$.

The process $\left(\left(X_{t}, L_{t}^{\bullet}\right), t \geq 0\right)$, where $L_{t}^{\bullet}=\left(L_{t}^{y}, y \in \mathbb{R}\right)$ denotes the local times process (in the space variable) at time $t$, for Brownian motion $\left(X_{t}, t \geq 0\right)$ is a Markov process taking values in $\mathbb{R} \times \widetilde{\Omega}=\mathbb{R} \times \mathcal{C}\left(\mathbb{R} \rightarrow \mathbb{R}_{+}\right)$. In fact, this process is the image of the process $\left(\mathcal{X}_{t}, t \geq 0\right)$ (see 1.3.1) by the application :

$$
H: \Omega \rightarrow \mathbb{R} \times \widetilde{\Omega}
$$

defined by :

$$
H\left(X_{t}, t \geq 0\right)=\left(X_{t}, L_{t}^{\bullet}\right)
$$


(and $H\left(X_{t}, t \geq 0\right)=(0,0)$ if the trajectory $\left(X_{t}, t \geq 0\right)$ admits no local time). Thus, as a Corollary of Theorem 1.3.1, the image of $\widetilde{\mathbf{W}}^{a, b}$ by $H$ is an invariant measure for the process $\left(\left(X_{t}, L_{t}^{\bullet}\right), t \geq 0\right)$. This image, which we denote by $\widetilde{\Lambda}^{a, b}$ is equal, from (1.3.6), to :

$$
\widetilde{\Lambda}^{a, b}=\frac{1}{2} \int_{\mathbb{R}} d x \int_{0}^{\infty} d u\left(a Q_{x, u}^{2,0}+b Q_{x, u}^{0,2}\right)
$$

Thus, we have obtained :

Theorem 1.3.2. The measure $\widetilde{\boldsymbol{\Lambda}}^{a, b}$ is an invariant measure for the process $\left(\left(X_{t}, L_{t}^{\bullet}\right), t \geq 0\right)$. We shall now give a different proof of Theorem 1.3.2 than the one we have just indicated. This proof has the further advantage that it hinges on arguments which shall be useful in the sequel. We begin with the :

Lemma 1.3.3 Let $q \in \mathcal{I}$

1) Define $\varphi_{q}^{+}(x):=\mathbf{W}_{x}^{+}\left(e^{-\frac{1}{2} A_{\infty}^{(q)}}\right)=\mathbf{W}_{x}\left(e^{-\frac{1}{2} A_{\infty}^{(q)}} 1_{\Gamma_{+}}\right)$. Then, $\varphi_{q}^{+}$is the unique solution of Sturm-Liouville equation:

$$
\begin{aligned}
& \varphi^{\prime \prime}=q \varphi \quad \text { with boundary conditions : } \\
& \varphi(x) \underset{x \rightarrow+\infty}{\sim} x \quad \varphi(x) \underset{x \rightarrow-\infty}{\longrightarrow} C:=\frac{1}{\int_{-\infty}^{\infty} \frac{d y}{\varphi_{q}^{2}(y)}}
\end{aligned}
$$

2) Define $\varphi_{q}^{-}(x):=\mathbf{W}_{x}^{-}\left(e^{-\frac{1}{2} A_{\infty}^{(q)}}\right)=\mathbf{W}_{x}\left(e^{-\frac{1}{2} A_{\infty}^{(q)}} 1_{\Gamma_{-}}\right)$. Then, $\varphi_{q}^{-}$is the unique solution of the Sturm-Liouville equation:

$$
\begin{aligned}
& \varphi^{\prime \prime}=q \varphi \quad \text { with boundary conditions : } \\
& \varphi(x) \underset{x \rightarrow-\infty}{\sim} x \quad \varphi(x) \underset{x \rightarrow+\infty}{\longrightarrow} C:=\frac{1}{\int_{-\infty}^{\infty} \frac{d y}{\varphi_{q}^{2}(y)}}
\end{aligned}
$$

\section{Proof of Lemma 1.3.3.}

It suffices, by symmetry, to prove point 1 . We have

$$
\begin{aligned}
\mathbf{W}_{x}\left(e^{-\frac{1}{2} A_{\infty}^{(q)}} 1_{\Gamma_{+}}\right) & =\varphi_{q}(x) W_{x, \infty}^{(q)}\left(\Gamma_{+}\right) \quad(\text { from }(1.1 .16)) \\
& =\lim _{\substack{a \rightarrow-\infty \\
b \rightarrow+\infty}} \varphi_{q}(x) W_{x, \infty}^{(q)}\left(T_{b}<T_{a}\right)
\end{aligned}
$$

But, from (1.1.14), this limit equals :

$$
\varphi_{q}(x) \frac{\gamma_{q}(x)-\gamma_{q}(-\infty)}{\varphi_{q}(\infty)-\gamma_{q}(-\infty)}:=\varphi_{q}(x) \frac{\gamma_{q}(x)-\alpha}{\beta-\alpha}
$$

where $\gamma_{q}$ is given by (1.1.14). Hence :

$$
\varphi_{q}^{+}(x)=\varphi_{q}(x) \frac{\gamma_{q}(x)-\alpha}{\beta-\alpha} .
$$


It remains to prove that $\varphi_{q}^{+}$satisfies the announced conditions. But (with $\gamma$ for $\gamma_{q}$ ):

$$
\begin{aligned}
\left(\varphi_{q}^{+}\right)^{\prime \prime}(x) & =\varphi_{q}^{\prime \prime}(x)\left(\frac{\gamma(x)-\alpha}{\beta-\alpha}\right)+2 \varphi_{q}^{\prime}(x) \frac{\gamma^{\prime}(x)}{\beta-\alpha}+\varphi_{q}(x) \frac{\gamma^{\prime \prime}(x)}{\beta-\alpha} \\
& =\varphi_{q}^{\prime \prime}(x)\left(\frac{\gamma(x)-\alpha}{\beta-\alpha}\right)+\frac{2 \varphi_{q}^{\prime}(x)}{\beta-\alpha} \frac{1}{\varphi_{q}^{2}(x)}+\frac{\varphi_{q}(x)}{\beta-\alpha}\left(-2 \frac{\varphi_{q}^{\prime}(x)}{\varphi_{q}^{3}(x)}\right) \\
& =\varphi_{q}^{\prime \prime}(x)\left(\frac{\gamma(x)-\alpha}{\beta-\alpha}\right)=q(x) \varphi_{q}(x) \frac{\gamma(x)-\alpha}{\beta-\alpha}=q(x) \varphi_{q}^{+}(x)
\end{aligned}
$$

On the other hand :

$$
\begin{aligned}
\varphi_{q}^{+}(x) & =\varphi_{q}(x) \frac{\gamma(x)-\gamma(-\infty)}{\gamma(\infty)-\gamma(-\infty)} \underset{x \rightarrow \infty}{\sim} \varphi_{q}(x) \underset{x \rightarrow \infty}{\sim} x \\
\varphi_{q}^{+}(x) & =\varphi_{q}(x) \frac{\int_{-\infty}^{x} \frac{d y}{\varphi_{q}^{2}(y)}}{\int_{-\infty}^{\infty} \frac{d y}{\varphi_{q}^{2}(y)}} \underset{x \rightarrow-\infty}{\sim} C \frac{\varphi_{q}(x)}{|x|} \underset{x \rightarrow-\infty}{\sim} C=\frac{1}{\int_{-\infty}^{\infty} \frac{d y}{\varphi_{q}^{2}(y)}}
\end{aligned}
$$

This proves Lemma 1.3.3.

\section{We now prove Theorem 1.3.2.}

Of course, by symmetry, it suffices to show that the measure : $\widetilde{\boldsymbol{\Lambda}}^{+}:=\int_{\mathbb{R}} d x \boldsymbol{\Lambda}_{x}^{+}$, where $\boldsymbol{\Lambda}_{x}^{+}$ is the image of $\mathbf{W}_{x}^{+}$by $\mathcal{L}$, is invariant for the process $\left(\left(X_{t}, L_{t}^{\bullet}\right), t \geq 0\right)$. We note that from (1.3.6), we have :

$$
\widetilde{\boldsymbol{\Lambda}}_{x}^{+}=\frac{1}{2} \int_{0}^{\infty} d u Q_{x, u}^{2,0}
$$

We denote by $\left(Q_{t}, t \geq 0\right)$ the semi-group which is associated to the process $\left(\left(X_{t}, L_{t}^{\bullet}\right), t \geq 0\right)$, and we consider $F: \mathbb{R} \times \widetilde{\Omega} \rightarrow \mathbb{R}_{+}$of the form :

$$
\begin{aligned}
F(x, l) & =f(x) \exp \left(-\frac{1}{2}<q, l>\right) \\
& =f(x) \exp \left(-\frac{1}{2} \int_{\mathbb{R}} l(y) q(y) d y\right)
\end{aligned}
$$

for $q \in \mathcal{I}$ and $f$ Borel, bounded. Then, for such an $F$, we obtain, by definition of the process $\left(\left(X_{t}, L_{t}^{\bullet}\right), t \geq 0\right)$ :

$$
Q_{t} F(x, l)=W\left(f\left(x+X_{t}\right) \exp \left\{-\frac{1}{2}<q, l>-\frac{1}{2} \int_{0}^{t} q\left(x+X_{s}\right) d s\right\}\right)
$$

Now, from the monotone class theorem, Theorem 1.3.2 shall be obtained once we show that:

$$
\int_{\mathbb{R}} d x \int_{\widetilde{\Omega}} \boldsymbol{\Lambda}_{x}^{+}(d l) Q_{t} F(x, l)=\int_{\mathbb{R}} d x \int_{\widetilde{\Omega}} \boldsymbol{\Lambda}_{x}^{+}(d l) F(x, l)
$$

for every $t \geq 0$. Indeed, from Lemma 1.3.3, we have :

$$
\begin{aligned}
\mathbf{W}_{x}^{+}\left(\exp -\frac{1}{2} A_{\infty}^{(q)}\right) & =\mathbf{W}_{x}\left(\exp \left(-\frac{1}{2} A_{\infty}^{(q)}\right) \cdot 1_{\Gamma_{+}}\right) \\
& =\int_{\widetilde{\Omega}} \boldsymbol{\Lambda}_{x}^{+}(d l) \exp \left(-\frac{1}{2}<q, l>\right)=\varphi_{q}^{+}(x)
\end{aligned}
$$


since $\boldsymbol{\Lambda}_{x}^{+}$is the image of $\mathbf{W}_{x}^{+}$by $\mathcal{L}$.

Thus, the left-hand side of (1.3.19) writes :

$$
\begin{aligned}
L H S & =<Q_{t} F, 1>\widetilde{\Lambda}^{+} \\
& =\int_{\mathbb{R}} d x \int_{\widetilde{\Omega}} \Lambda_{x}^{+}(d l) W\left(f\left(x+X_{t}\right) e^{-\frac{1}{2}<q, l>-\frac{1}{2} \int_{0}^{t} q\left(x+X_{s}\right) d s}\right) \\
& =W\left(\int_{\mathbb{R}} d x \varphi_{q}^{+} f\left(x+X_{t}\right) \exp \left(-\frac{1}{2} \int_{0}^{t} q\left(x+X_{s}\right) d s\right)\right)
\end{aligned}
$$

from Fubini and (1.3.20)

$$
=\int_{\mathbb{R}} f(y) d y W\left(\varphi_{q}\left(y-X_{t}\right) \exp \left(-\frac{1}{2} \int_{0}^{t} q\left(y-X_{t}+X_{s}\right) d s\right)\right.
$$

after making the change of variables $x+X_{t}=y$. On the other hand, the right-hand side of (1.3.19) equals :

$$
\begin{aligned}
R H S & =\int_{\mathbb{R}} d y \int_{\widetilde{\Omega}} \boldsymbol{\Lambda}_{y}^{+}(d l) f(y) \exp \left(-\frac{1}{2}<l, q>\right) \\
& =\int_{\mathbb{R}} f(y) \varphi_{q}^{+}(y) d y
\end{aligned}
$$

from (1.3.20). Thus, Theorem 1.3.2 is an immediate consequence of the following :

Lemma 1.3.4. For every $q \in \mathcal{I}, x$ real and $t \geq 0$ :

$$
W\left(\varphi_{q}^{+}\left(y-X_{t}\right) \exp \left(-\frac{1}{2} \int_{0}^{t} q\left(y-X_{t}+X_{s}\right) d s\right)=\varphi_{q}^{+}(y)\right.
$$

Furthermore, (1.3.23) is also true when $\varphi_{q}^{+}$is replaced by $\varphi_{q}^{-}$or $\varphi_{q}$.

\section{Proof of Lemma 1.3.4.}

$$
\begin{aligned}
& W\left(\varphi_{q}^{+}\left(y-X_{t}\right) \exp \left(-\frac{1}{2} \int_{0}^{t} q\left(y-X_{t}+X_{s}\right) d s\right)\right) \\
& \quad=W\left(\varphi_{q}^{+}\left(y-X_{t}\right) \exp \left(-\frac{1}{2} \int_{0}^{t} q\left(y-X_{t}+X_{t-r}\right) d r\right)\right)
\end{aligned}
$$

(after making the change of variables $r=t-s$ ).

$$
=W\left(\varphi_{q}^{+}\left(y-X_{t}\right) \exp \left(-\frac{1}{2} \int_{0}^{t} q\left(y-X_{r}\right) d r\right)\right)
$$

(since the process $\left(X_{t}-X_{t-r}, 0 \leq r \leq t\right)$ has the same law as $\left(X_{r}, 0 \leq r \leq t\right)$ )

$$
=W_{y}\left(\varphi_{q}^{+}\left(X_{t}\right) \exp \left(-\frac{1}{2} \int_{0}^{t} q\left(X_{r}\right) d r\right)\right)
$$

(since $\left(-X_{r}, r \geq 0\right)$ has the same law as $\left(X_{r}, r \geq 0\right)$ )

$$
=\varphi_{q}^{+}(y)
$$


because, from (1.3.10) and Itô's formula, $\left(\varphi_{q}^{+}\left(X_{t}\right) \exp \left(-\frac{1}{2} \int_{0}^{t} q\left(X_{r}\right) d r\right), t \geq 0\right)$ is a $\left(\left(\mathcal{F}_{t}, t \geq 0\right), W_{y}\right)$ martingale.

\section{Remark 1.3.5.}

1) We denote by $\mathcal{G}$ the infinitesimal generator of the process $\left(\left(X_{t},, L_{t}^{\bullet}\right), t \geq 0\right)$. For a function $F$ of the form given by (1.3.17), we obtain :

$$
\begin{aligned}
& \mathcal{G} F(x, l) \\
& \quad=\left.\frac{d}{d s}\right|_{s=0} Q_{s} F(x, l) \\
& \quad=\left.\frac{d}{d s}\right|_{s=0} W\left(f\left(x+X_{s}\right) \exp \left(-\frac{1}{2}<q, l>-\frac{1}{2} \int_{0}^{s} q\left(x+X_{r}\right) d r\right)\right) \\
& (\text { from }(1.3 .18)) \\
& \quad=\exp \left(-\frac{1}{2}<q, l>\right) \cdot\left[\frac{1}{2} f^{\prime \prime}(x)-\frac{1}{2} q(x) f(x)\right] \\
& \quad=\frac{1}{2} \frac{\partial^{2} F}{\partial x^{2}}(x, l)-\frac{1}{2} q(x) F(x, l)
\end{aligned}
$$

Another way to prove Theorem 1.3.2 consists in showing that, for every $F$ of the form (1.3.17), we have :

$$
<\mathcal{G F}, 1>_{\widetilde{\mathbf{\Lambda}}^{a, b}}=0
$$

\section{Let us prove (1.3.26).}

By symmetry, it suffices to prove (1.3.26) by replacing $\widetilde{\boldsymbol{\Lambda}}^{c, b}$ by $\widetilde{\boldsymbol{\Lambda}}^{+}$. Now, we obtain, for $F$ of the form (1.3.17) with $f$ of class $C^{2}$, with compact support :

$$
\begin{aligned}
<\mathcal{G F}, 1_{\widetilde{\boldsymbol{\Lambda}}^{+}}> & =\int_{\mathbb{R}} d x \int_{\widetilde{\Omega}} \widetilde{\boldsymbol{\Lambda}}_{x}^{+}(d l) e^{-\frac{1}{2}<q, l>}\left(\frac{1}{2} f^{\prime \prime}(x)-\frac{1}{2} q(x) f(x)\right) \\
(\text { from }(1.3 .24)) & \\
= & \int_{\mathbb{R}} \varphi_{q}^{+}(x) d x\left(\frac{1}{2} f^{\prime \prime}(x)-\frac{1}{2} q(x) d(x)\right)
\end{aligned}
$$

(from Lemma 1.3.3)

$$
=\int_{\mathbb{R}} \frac{1}{2} f(x)\left[\varphi_{q}^{+}(x)-q(x) \varphi_{q}^{+}(x)\right]
$$

(after integrating by parts)

$$
=0 \quad \text { (from Lemma 1.3.3.) }
$$

2) Theorem 1.3.2 invites to ask the following question : is the process $\left(\left(X_{t}, L_{t}^{\bullet}\right), t \geq 0\right)$ reversible with respect to the measure $\widetilde{\boldsymbol{\Lambda}}^{a, b}$, i.e. : does the following hold :

$$
<Q_{s} F, G>_{\widetilde{\mathbf{\Lambda}}^{a, b}}=<F, Q_{s} G>_{\widetilde{\mathbf{\Lambda}}^{a, b}}
$$

for every $F, G: \mathbb{R} \times \Omega \rightarrow \mathbb{R}_{+}$measurable and positive ? The answer to this question is negative. In particular, the operator $L$ is not symmetric, i.e., in general :

$$
<L F, G>_{\widetilde{\mathbf{\Lambda}}^{a, b}} \neq<F, L G>_{\widetilde{\boldsymbol{\Lambda}}^{a, b}}
$$


We now show (1.3.28), with $F(x, l)=f(x) \exp \left(-\frac{1}{2}<q, l>\right), G(x, l)=g(x), \quad \widetilde{\boldsymbol{\Lambda}}^{a, b}=\widetilde{\boldsymbol{\Lambda}}$ $:=\widetilde{\boldsymbol{\Lambda}}^{1,1}$. Assuming that the equality would hold in (1.3.28), we would obtain, after an elementary computation :

$$
\begin{aligned}
<\mathcal{G} F, G>_{\widetilde{\Lambda}} & =\int_{\mathbb{R}} \varphi_{q}(x) g(x)\left(\frac{1}{2} f^{\prime \prime}(x)-\frac{1}{2} q(x) f(x)\right) d x \\
& =\int_{\mathbb{R}} \varphi_{q}(x) f(x) \frac{1}{2} g^{\prime \prime}(x) d x=<F, \mathcal{G} G>_{\widetilde{\Lambda}}
\end{aligned}
$$

Thus, the preceding equality would imply, after integrating by parts and use of the relation $\varphi_{q}^{\prime \prime}=q \varphi_{q}$ :

$$
-2 q(x) \varphi_{q}(x) f(x)=2 \varphi_{q}^{\prime}(x) f^{\prime}(x)
$$

for every $f$ in class $C^{2}$, with compact support, which is absurd.

3) Of course, the preceding point implies that the measure $\widetilde{\mathbf{W}}^{a, b}$ which is invariant for the process $\left(\mathcal{X}_{t}, t \geq 0\right)$ is not reversible.

4) The following relation, which has been obtained from Lemma 1.3 .3 and the definition of $\Lambda_{x}^{ \pm}:$

$$
W_{x}\left[\varphi_{q}^{ \pm}\left(X_{t}\right) \exp \left(-\frac{1}{2} A_{t}^{(q)}\right)\right]=\int_{\widetilde{\Omega}} \widetilde{\Lambda}_{x}^{ \pm}(d l) \exp \left(-\frac{1}{2}<q, l>\right)
$$

is a particular case of the following result, which is found in ([RY, M], Chap. 2) :

Let $F: \widetilde{\Omega} \rightarrow \mathbb{R}_{+}$measurable, and "sub-exponential at infinity, (i.e. : there exixts $q \in \mathcal{I}$ and $C>0$ such that, for every $l \in \widetilde{\Omega}, F(l) \leq C \exp (-<q, l>))$, then :

$$
\left(\int_{\widetilde{\Omega}} \widetilde{\Lambda}_{X_{t}}^{ \pm}(d l) F\left(l+L_{t}^{\bullet}\right), t \geq 0\right)
$$

is a $\left(\left(\mathcal{F}_{t}, t \geq 0\right), W\right)$ martingale ; hence :

$$
\begin{aligned}
W_{x}\left(\int_{\widetilde{\Omega}} \widetilde{\Lambda}_{X_{t}}^{ \pm}(d l) F\left(l+L_{t}^{\bullet}\right)\right) & =W_{x}\left(\int_{\widetilde{\Omega}} \widetilde{\Lambda}_{X_{0}}^{ \pm}(d l) F(l)\right) \\
& =\int_{\widetilde{\Omega}} \widetilde{\Lambda}_{x}^{ \pm}(d l) F(l)
\end{aligned}
$$

In particular, if $F(l)=\exp \left(-\frac{1}{2}<q, l>\right),(1.3 .31)$ becomes :

$$
\begin{aligned}
\int_{\widetilde{\Omega}} \widetilde{\Lambda}_{X_{t}}^{ \pm}(d l) F\left(l+L_{t}^{\bullet}\right) & =\int_{\widetilde{\Omega}} \widetilde{\Lambda}_{X_{t}}^{ \pm}(d l) \exp \left(-\frac{1}{2}<q, l>-\frac{1}{2} \int_{\mathbb{R}} q(x) L_{t}^{x} d x\right) \\
& =\varphi_{q}^{ \pm}\left(X_{t}\right) \exp \left(-\frac{1}{2} A_{t}^{(q)}\right)
\end{aligned}
$$

Thus, when : $F(l)=\exp \left(-\frac{1}{2}<q, l>\right),(1.3 .31)$ is nothing else but (1.3.29) since :

$$
W_{x}\left(\varphi_{q}^{ \pm}\left(X_{t}\right) \exp \left(-\frac{1}{2} A_{t}^{(q)}\right)\right)=\varphi_{q}^{ \pm}(x) .
$$


5) Theorem 1.3.2 also invites to ask the question : are the measures $\left(\widetilde{\boldsymbol{\Lambda}}^{a, b}, a, b \geq 0\right)$ the only invariant measures of the process $\left(\left(X_{t}, L_{t}^{\bullet}\right), t \geq 0\right)$. Here is a partial answer to this question. Let $\widehat{\Lambda}$ be an invariant measure for this process.

i) Since the first component of $\left(\left(X_{t}, L_{t}^{\bullet}\right), t \geq 0\right)$ is a Brownian motion, and that process admits as its only invariant measure (up to a multiplicative factor) the Lebesgue measure on $\mathbb{R}$, the measure $\widehat{\Lambda}$ admits a desintegration of the form :

$$
\widehat{\boldsymbol{\Lambda}}(d x, d l)=d x \widehat{\boldsymbol{\Lambda}}_{x}(d l)
$$

and, denoting by $\widehat{\varphi}_{q}$ the function defined by :

$$
\widehat{\varphi}_{q}(x)=\widehat{\boldsymbol{\Lambda}}_{x}\left(\exp -\frac{1}{2}<q, l>\right)
$$

the computation which lead to (1.3.21) and to (1.3.22) imply, if $\widehat{\boldsymbol{\Lambda}}$ is invariant :

$$
\widehat{\varphi}_{q}(x)=W_{x}\left(\widehat{\varphi}_{q}\left(X_{t}\right) \exp \left(-\frac{1}{2} \int_{0}^{t} q\left(X_{s}\right) d s\right)\right)
$$

It follows from this formula, using Itô's Lemma, that :

$$
\widehat{\varphi}_{q}^{\prime \prime}=q \widehat{\varphi}_{q}
$$

The vector space of the solutions of the Sturm-Liouville equation has dimension 2 ; hence, there exist two constants $C_{ \pm}(q)$ such that :

$$
\widehat{\varphi}_{q}(x)=C_{+}(q) \varphi_{q}^{+}(x)+C_{-}(q) \varphi_{q}^{-}(x)
$$

ii) The invariant measure $\widetilde{\boldsymbol{\Lambda}}^{a, b}$ which we described in Theorem 1.1.2, and which writes :

$$
\begin{aligned}
\widetilde{\boldsymbol{\Lambda}}^{a, b} & =\frac{1}{2} \int_{\mathbb{R}} d x\left(a \boldsymbol{\Lambda}_{x}^{+}+b \boldsymbol{\Lambda}_{x}^{-}\right) \\
& =\int_{\mathbb{R}} d x \boldsymbol{\Lambda}_{x}^{a, b} \\
\text { with } \boldsymbol{\Lambda}_{x}^{a, b} & :=\frac{1}{2}\left(a \boldsymbol{\Lambda}_{x}^{+}+b \boldsymbol{\Lambda}_{x}^{-}\right)
\end{aligned}
$$

enjoys the following property :

$$
\lim _{x \rightarrow+\infty} \frac{1}{x} \boldsymbol{\Lambda}_{x}^{a, b}\left(\exp -\frac{1}{2}<q, l>\right) \quad \text { and } \lim _{x \rightarrow-\infty} \frac{1}{|x|} \boldsymbol{\Lambda}_{x}^{a, b}\left(\exp -\frac{1}{2}<q, l>\right)
$$

do not depend on $q \in \mathcal{I}$. Indeed,

$$
\frac{1}{x} \boldsymbol{\Lambda}_{x}^{a, b}\left(\exp -\frac{1}{2}<q, l>\right)=\frac{1}{2 x}\left(a \varphi_{q}^{+}(x)+b \varphi_{q}^{-}(x)\right) \underset{x \rightarrow \infty}{\longrightarrow} \frac{a}{2}
$$

from Lemma 1.3 .3 and $\frac{1}{|x|} \Lambda_{x}^{a, b}\left(\exp -\frac{1}{2}<q, l>\right) \underset{x \rightarrow-\infty}{\longrightarrow} \frac{b}{2}$. 
iii) We now assume that the invariant measure $\widehat{\boldsymbol{\Lambda}}$, which equals : $\widehat{\boldsymbol{\Lambda}}(d s, d l)=d x \widehat{\boldsymbol{\Lambda}}_{x}(d l)$ also satisfies that both limits :

$$
\lim _{x \rightarrow \infty} \frac{1}{x} \widehat{\boldsymbol{\Lambda}}_{x}\left(\exp -\frac{1}{2}<q, l>\right) \text { and } \lim _{x \rightarrow-\infty} \frac{1}{|x|} \widehat{\boldsymbol{\Lambda}}_{x}\left(\exp -\frac{1}{2}<q, l>\right)
$$

exist and do not depend on $q \in \mathcal{I}$. Then, there exist $a$ and $b$ positive, such that : $\widehat{\Lambda}=\widehat{\boldsymbol{\Lambda}}^{a, b}$. Indeed, from (1.3.35), together with Lemma 1.3.3 and (1.3.33), we have :

$$
\begin{aligned}
\lim _{x \rightarrow \infty} & \frac{1}{x} \widehat{\boldsymbol{\Lambda}}_{x}\left(\exp \left(-\frac{1}{2}<q, l>\right)\right)=\lim _{x \rightarrow \infty} \frac{\widehat{\varphi}_{q}(x)}{x} \\
= & \lim _{x \rightarrow \infty} \frac{C_{+}(q) \varphi_{q}^{+}(x)+C_{-}(q) \varphi_{q}^{-}(x)}{x}=C_{+}(q)
\end{aligned}
$$

Thus, $C_{+}(q)$ (and $C_{-}(q)$, by symmetry) are constants, which we shall denote respectively as $\frac{a}{2}$ and $\frac{b}{2}$. Thus, we have :

$$
\begin{aligned}
\widehat{\boldsymbol{\Lambda}}_{x}\left(\exp -\frac{1}{2}<q, l>\right) & =\frac{a}{2} \varphi_{q}^{+}(x)+\frac{b}{2} \varphi_{q}^{-}(x) \\
& =\boldsymbol{\Lambda}_{x}^{a, b}\left(e^{-\frac{1}{2}<q, l>}\right)
\end{aligned}
$$

Hence : $\widehat{\boldsymbol{\Lambda}}_{x}=\boldsymbol{\Lambda}_{x}^{a, b}$ and $\widehat{\boldsymbol{\Lambda}}=\widetilde{\boldsymbol{\Lambda}}^{a, b}$.

1.3.4 Invariant measures of the process $\left(L_{t}^{X_{t^{-}}}, t \geq 0\right)$.

1.3.4.1 For $t \geq 0$, we define the random measure $\mu_{t}$ via :

$$
\mu_{t}(f)=\int_{0}^{t} f\left(X_{t}-X_{s}\right) d s
$$

with $f$ positive, continuous and bounded. It is proven in $[\mathrm{DM}, \mathrm{Y}]$ that $\left(\mu_{t}, t \geq 0\right)$ is a Markov process taking values in the space of positive measures on $(\mathbb{R}, \mathcal{B}(\mathbb{R}))$. Due to the density of occupation formula, we may write (1.3.40) in the form :

$$
\begin{aligned}
\mu_{t}(f) & =\int_{\mathbb{R}} f\left(X_{t}-y\right) L_{t}^{y} d y \\
& =\int_{\mathbb{R}} f(z) L_{t}^{X_{t}-z} d z
\end{aligned}
$$

We deduce that :

$$
\mu_{t}(d z)=L_{t}^{X_{t}-z} d z
$$

Hence, rather than working in the space of measures on $\mathbb{R}$, we shall consider the Markov process $\left(L_{t}^{X_{t}-\bullet}, t \geq 0\right)$ which takes values in $\widetilde{\Omega}=\mathcal{C}(\mathbb{R} \rightarrow \mathbb{R})$.

1.3.4.2 Of course, this Markov process is the image of the process $\left(\left(X_{t}, L_{t}^{\bullet}\right), t \geq 0\right)$ by the application :

$$
\theta: \mathbb{R} \times \widetilde{\Omega} \rightarrow \widetilde{\Omega}
$$

defined by :

$$
\theta(x, l)(y)=l(x-y) \quad x, y \in \mathbb{R}, l \in \widetilde{\Omega}
$$


This application $\theta$ is not bijective since :

$$
\theta(x, l)=\theta\left(x^{\prime}, l^{\prime}\right)
$$

as soon as :

$$
l\left(x-x^{\prime}+z\right)=l^{\prime}(z)
$$

for every $z \in \mathbb{R}$ i.e. : as soon as $l^{\prime}$ is an adequate translate of $l$.

i) We begin by verifying directly, i.e. : without using the result of Donati-Martin-Yor recalled above - that the process $\left(L_{t}^{X_{t}-\bullet}, t \geq 0\right)$, which takes values in $\mathcal{C}\left(\mathbb{R} \rightarrow \mathbb{R}_{+}\right)$is Markovian, in the natural filtration of the process $\left(\left(X_{t}, L_{t}^{\bullet}\right), t \geq 0\right)$. For this purpose, using Dynkin's [Dyn] criterion, and denoting by $\left(Q_{t}, t \geq 0\right)$ the semi-group associated to the process $\left(\left(X_{t}, L_{t}^{\bullet}\right), t \geq\right.$ $0)$, one needs to verify that :

$$
Q_{t}(F \circ \theta)(x, l)=Q_{t}(F \circ \theta)\left(x^{\prime}, l^{\prime}\right)
$$

for every $t \geq 0$ and $F: \widetilde{\Omega} \rightarrow \mathbb{R}_{+}$measurable, as soon as :

$$
\theta(x, l)=\theta\left(x^{\prime}, l^{\prime}\right)
$$

Of course, from the Monotone Class Theorem, it suffices to prove (1.3.45) for $F$ of the form $F_{q}, q \in \mathcal{I}$, with :

$$
F_{q}(l):=\exp \left(-\frac{1}{2}<q, l>\right) \quad(l \in \widetilde{\Omega})
$$

We have, from (1.3.43) :

$$
\begin{aligned}
F_{q} \circ \theta(x, l)= & \exp \left(-\frac{1}{2}<q, l(x-\cdot>)\right. \\
= & \exp \left(-\frac{1}{2} \int_{\mathbb{R}} q(y) l(x-y) d y\right)=\exp \left(-\frac{1}{2}<\stackrel{\vee}{q_{x}}, l>\right) \\
\text { with } \quad & \stackrel{\vee}{q_{x}}(y)=q(x-y)
\end{aligned}
$$

Thus, from (1.3.18) :

$$
Q_{t}\left(F_{q} \circ \theta\right)(x, l)=W\left(\exp \left(-\frac{1}{2}<\stackrel{\vee}{q}_{x+X_{t}}, l>-\frac{1}{2} \int_{0}^{t} q\left(x+X_{t}-\left(x+X_{r}\right) d r\right)\right)\right.
$$

However :

$$
\begin{aligned}
\left\langle\stackrel{\vee}{q}_{x+X_{t}, l}, l>\right. & =\int_{\mathbb{R}} q\left(x+X_{t}-y\right) l(y) d y \\
& =\int_{\mathbb{R}} q\left(X_{t}+z\right) l(x-z) d z
\end{aligned}
$$

Thus, from (1.3.44), if $\theta(x, l)=\theta\left(x^{\prime}, l^{\prime}\right)$, we have :

$$
l(x-z)=l^{\prime}\left(x^{\prime}-z\right) \quad \text { hence } \quad<\stackrel{\vee}{q}_{x+X_{t}}, l>=<\stackrel{\vee}{q}_{x^{\prime}+X_{t}}, l>
$$


It now follows from (1.3.49) that :

$$
Q_{t}\left(F_{q} \circ \theta\right)(x, l)=Q_{t}\left(F_{q} \circ \theta\right)\left(x^{\prime}, l^{\prime}\right)
$$

1.3.4.3 Invariant measures for the process $\left(L_{t}^{X_{t}-\bullet}, t \geq 0\right)$.

Of course, from Theorem 1.3.2, the image of $\widetilde{\Lambda}^{a, b}$ by $\theta$ (defined by (1.3.43)) is an invariant measure for the process $\left(L_{t}^{X_{t}-\bullet}, t \geq 0\right)$. Unfortunately, an elementary computation shows that this measure is identically infinite. Thus, we need to find directly - without refering to $\widetilde{\Lambda}^{a, b}$ - invariant measures for $\left(L_{t}^{X_{t}-\bullet}, t \geq 0\right)$.

Theorem 1.3.6. Let $a, b \geq 0$, and:

$$
\boldsymbol{\Lambda}^{a, b}:=a \Lambda_{0}^{+}+b \boldsymbol{\Lambda}_{0}^{-}
$$

Then, $\boldsymbol{\Lambda}^{a, b}$ is an invariant measure for $\left(L_{t}^{X_{t}-\bullet}, t \geq 0\right)$.

We recall that $\boldsymbol{\Lambda}_{0}^{ \pm}$is the image of $\mathbf{W}_{0}^{ \pm}=\mathbf{W}^{ \pm}$by the application $\mathcal{L}$. In particular :

$$
\boldsymbol{\Lambda}_{0}^{ \pm}(\exp -<q, l>)=\varphi_{q}^{ \pm}(0) \quad(q \in \mathcal{I})
$$

We now show Theorem 1.3.6.

We denote $\left(\bar{Q}_{s}, s \geq 0\right)$ the semi-group associated to the process $\left(L_{t}^{X_{t}-\bullet}, t \geq 0\right)$. Thus, we have, for (1.3.49) :

$$
\bar{Q}_{s}\left(F_{q}\right)(l)=W\left(\exp \left(-\frac{1}{2}<q\left(X_{s}+\cdot, l>-\frac{1}{2} \int_{0}^{s} q\left(X_{s}-X_{r}\right) d r\right)\right)\right.
$$

with : $F_{q}(l)=\exp \left(-\frac{1}{2}<q, l>\right)$.

On the other hand, by symmetry, it suffices to show that the measure $\boldsymbol{\Lambda}^{+}:=\boldsymbol{\Lambda}_{0}^{+}$is invariant for $\left(L_{t}^{X_{t}-\bullet}, t \geq 0\right)$. We compute :

$$
\begin{aligned}
\int_{\widetilde{\Omega}} \boldsymbol{\Lambda}^{+}(d l)\left(\bar{Q}_{s}\left(F_{q}\right)\right)(l) \\
\quad=\int_{\widetilde{\Omega}} \boldsymbol{\Lambda}^{+}(d l) W\left(\exp \left(-\frac{1}{2}<q\left(X_{s}+\bullet\right), l>-\frac{1}{2} \int_{0}^{s} q\left(X_{s}-X_{r}\right) d r\right)\right) \\
\quad=W\left\{\left(\exp -\frac{1}{2} \int_{0}^{s} q\left(X_{s}-X_{r}\right) d r\right) \cdot \int_{\widetilde{\Omega}} \boldsymbol{\Lambda}^{+}(d l) \exp \left(-\frac{1}{2}<q\left(X_{s}+\bullet, l>\right)\right)\right\}
\end{aligned}
$$

(from Fubini)

$$
=W\left\{\exp \left(-\frac{1}{2} \int_{0}^{s} q\left(X_{s}-X_{r}\right) d r\right) \cdot \varphi_{q\left(X_{s}+\bullet\right)}^{+}(0)\right\}
$$

(from (1.3.52)). Now, it is easy to check that :

$$
\varphi_{q\left(X_{s}+\bullet\right)}^{+}(0)=\varphi_{q}^{+}\left(X_{s}\right)
$$

Thus :

$$
\begin{aligned}
\int_{\widetilde{\Omega}} \boldsymbol{\Lambda}^{+}(d l)\left(\widehat{Q}_{s}\left(F_{q}\right)(l)\right) & =W\left(\varphi_{q}^{+}\left(X_{s}\right) \exp \left(-\frac{1}{2} \int_{0}^{s} q\left(X_{s}-X_{r}\right) d r\right)\right) \\
& =\varphi_{q}^{+}(0)
\end{aligned}
$$


from Lemma 1.3.4 (replacing $\left(X_{t}, t \geq 0\right)$ by $\left(-X_{t}, t \geq 0\right)$ )

$$
=\int_{\widetilde{\Omega}} \boldsymbol{\Lambda}^{+}(d l) F_{q}(l) \quad(\text { from }(1.3 .52))
$$

This is Theorem 1.3.6.

\section{Remark 1.3.7.}

1) Arguing as in point 2 of Remark 1.3.5, it is easily shown that none of the measures $\boldsymbol{\Lambda}^{a, b}$ is reversible for the process $\left(L_{t}^{X_{t}-\bullet}, t \geq 0\right)$.

2) Here is another way to prove that $\boldsymbol{\Lambda}^{a, b}$ is invariant. (We give the details for $\boldsymbol{\Lambda}^{+}$). We have, with $F_{q}(l)=\exp -\frac{1}{2}<q, l>$, from (1.3.53) :

$$
\bar{Q}_{s}\left(F_{q}\right)(l)=W\left(\exp \left(-\frac{1}{2}<q\left(X_{s}+\cdot, l>-\frac{1}{2} \int_{0}^{s} q\left(X_{r}\right) d r\right)\right)\right.
$$

We proceeded from (1.3.53) to (1.3.55) by making the change of variable $r=s-u$ and using the fact that, under $W,\left(X_{s}-X_{s-r}, r \leq s\right) \stackrel{\text { (law) }}{=}\left(X_{r}, r \leq s\right)$. Thus, denoting by $\overline{\mathcal{G}}$ the infinitesimal generator of the semi-group $\left(\bar{Q}_{s}, s \geq 0\right)$, we obtain :

$$
\begin{aligned}
\overline{\mathcal{G}} F_{q}(l) & =\left.\frac{d}{d s}\right|_{s=0} \bar{Q}_{s}\left(F_{q}\right)(l) \\
& =\left.\frac{d}{d s}\right|_{s=0} W\left[g\left(X_{s}\right) \exp \left(-\frac{1}{2} \int_{0}^{s} q\left(X_{s}\right) d r\right)\right] \\
(\text { with } g(x) & :=\exp \left(-\frac{1}{2}<q(x+\cdot, l>)\right) \\
& =\frac{1}{2} g^{\prime \prime}(0)-\frac{1}{2} q(0) g(0) \\
& =\frac{1}{2}\left[\frac { \partial ^ { 2 } } { \partial x ^ { 2 } } | _ { x = 0 } \left(\exp \left(-\frac{1}{2}<q(x+\cdot, l)>\right)-q(0) \exp \left(-\frac{1}{2}<q, l>\right)\right.\right.
\end{aligned}
$$

Thus :

$$
\begin{aligned}
<\overline{\mathcal{G}} F_{q}, 1>_{\Lambda^{+}} & =\int_{\widetilde{\Omega}} \overline{\mathcal{G}} F_{q}(l) \Lambda^{+}(d l) \\
& =\frac{1}{2} \int_{\widetilde{\Omega}} \boldsymbol{\Lambda}^{+}(d l)\left(\left.\frac{\partial^{2}}{\partial x^{2}}\right|_{x=0} \exp \left(-\frac{1}{2}<q(x+\cdot), l>\right)-q(0) \exp \left(-\frac{1}{2}<q, l>\right)\right) \\
& =\frac{1}{2}\left(\varphi_{q}^{+\prime \prime}(0)-q(0) \varphi_{q}^{+}(0)\right)=0
\end{aligned}
$$

after interverting the second derivative and integration with respect to $\boldsymbol{\Lambda}^{+}(d l)$, using Lemma 1.3.3 and the fact that $\varphi_{q(x+\cdot)}^{+}(0)=\varphi_{q}^{+}(x)$. From relation (1.3.56), we deduce of course that : $<\bar{Q}_{s} F_{q}, 1>_{\boldsymbol{\Lambda}^{+}}=<F_{q}, 1>_{\boldsymbol{\Lambda}^{+}}$, i.e. that $\boldsymbol{\Lambda}^{+}$is invariant. 


\section{Chapter 2. Existence and properties of the measure $\mathbf{W}^{(2)}$.}

We shall now establish a number of results similar to those of Chapter 1, but this time $\left(X_{t}, t \geq 0\right)$ is a 2 -dimensional Brownian motion.

\subsection{Existence of $\mathbf{W}^{(2)}$.}

2.1.1 Notations and Feynman-Kac penalisations in two dimensions.

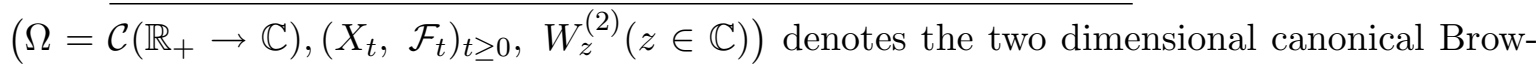
nian motion, which takes its values in $\mathbb{C}$. We write $W^{(2)}$ for $W_{0}^{(2)}$. $\mathcal{I}$ denotes here the set of positive Radon measures on $\mathbb{C}$ admitting a density $q$ with compact support and such that $\int q(x) d x>0$. Define :

$$
A_{t}^{(q)}:=\int_{0}^{t} q\left(X_{s}\right) d s
$$

Here is the analogue in dimension 2 of Theorem 1.1.1. A proof of this Theorem (in dimension 2 ) is found in [RVY, VI].

Theorem 2.1.1. Let $q \in \mathcal{I}$ and, for every $t \geq 0$ and $z \in \mathbb{C}$ :

$$
W_{z, t}^{(2, q)}:=\frac{\exp \left(-\frac{1}{2} A_{t}^{(q)}\right)}{Z_{z, t}^{(2, q)}} \cdot W_{z}^{(2)}
$$

with

$$
Z_{z, t}^{(2, q)}:=W_{z}^{(2)}\left(\exp -\frac{1}{2} A_{t}^{(q)}\right)
$$

1) For every $s \geq 0$ and $\Gamma_{s} \in b\left(\mathcal{F}_{s}\right)$ :

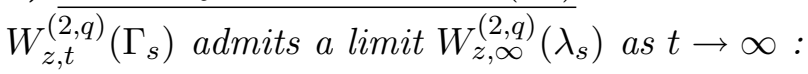

$$
W_{z, t}^{(2, q)}\left(\Gamma_{s}\right) \underset{t \rightarrow \infty}{\longrightarrow} W_{z, \infty}^{(2, q)}\left(\Gamma_{s}\right)
$$

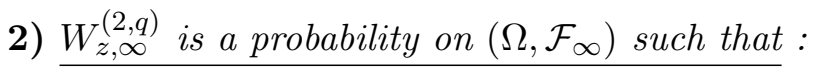

$$
\left.W_{z, \infty}^{(2, q)}\right|_{\mathcal{F}_{s}}=\left.M_{s}^{(2, q)} \cdot W_{z}^{(2)}\right|_{\mathcal{F}_{s}}
$$

where $\left(M_{s}^{(2, q)}, s \geq 0\right)$ is the $\left(\left(\mathcal{F}_{s}, s \geq 0\right), W_{z}^{(2)}\right)$ martingale defined by :

$$
M_{s}^{(2, q)}=\frac{\varphi_{q}\left(X_{s}\right)}{\varphi_{q}(z)} \exp \left(-\frac{1}{2} A_{s}^{(q)}\right)
$$

3) The function $\varphi_{q}: \mathbb{C} \rightarrow \mathbb{R}_{+}$featured in (2.1.5) is strictly positive, continuous and satisfies:

$$
\varphi_{q}(z) \underset{|z| \rightarrow \infty}{\sim} \frac{1}{\pi} \log (|z|)
$$

It may be defined via one or the other of the following descriptions :

i) $\varphi_{q}$ is the unique solution of the Sturm-Liouville equation:

$$
\Delta \varphi=q \cdot \varphi \quad \text { (in the sense of Schwartz distributions) }
$$


which satisfies the limiting condition:

$$
|z| \frac{\partial \varphi}{\partial r}(z) \underset{r \rightarrow \infty}{\longrightarrow} \frac{1}{\pi} \quad(r=|z|)
$$

ii) $\quad \frac{1}{2 \pi}(\log t) W_{z}^{(2)}\left(\exp \left(-\frac{1}{2} A_{t}^{(q)}\right)\right) \underset{r \rightarrow \infty}{\longrightarrow} \varphi_{q}(z)$

3) Under the family of probabilities $\left(W_{z, \infty}^{(2, q)}, z \in \mathbb{C}\right)$, the canonical process $\left(X_{t}, t \geq 0\right)$ is a transient diffusion. More precisely, there exists a $\left(\Omega,\left(\mathcal{F}_{t}, t \geq 0\right), W_{z, \infty}^{(2, q)}\right)$ Brownian motion $\left(B_{t}, t \geq 0\right)$ valued in $\mathbb{C}$ and starting from 0 such that :

$$
X_{t}=z+B_{t}+\int_{0}^{t} \frac{\nabla \varphi_{q}}{\varphi_{q}}\left(X_{s}\right) d s
$$

2.1.2 Existence of the measure $\mathbf{W}^{(2)}$.

Theorem 2.1.2. There exists on $\left(\Omega=\mathcal{C}\left(\mathbb{R}_{+} \rightarrow \mathbb{C}\right), \mathcal{F}_{\infty}\right)$ a $\sigma$-finite and positive measure $\mathbf{W}^{(2)}$ (with infinite total mass) such that, for every $q \in \mathcal{I}$ :

$$
\mathbf{W}^{(2)}=\varphi_{q}(0) \exp \left(+\frac{1}{2} A_{\infty}^{(q)}\right) \cdot W_{\infty}^{(2, q)}
$$

In other terms, the RHS of (2.1.10) does not depend on $q \in \mathcal{I}$.

In fact, just as in the case of dimension 1 , we show for every $z \in \mathbb{C}$, the existence of a measure $\mathbf{W}_{z}^{(2)}$, this measure being defined by :

$$
\mathbf{W}_{z}^{(2)}\left(F\left(X_{s}, s \geq 0\right)\right)=\mathbf{W}^{(2)}\left(F\left(z+X_{s}, s \geq 0\right)\right)
$$

\section{Proof of Theorem 2.1.2.}

i) Point 1) consists in showing that $\varphi_{q}(0) \exp \left(+\frac{1}{2} A_{\infty}^{(q)}\right) \cdot W_{\infty}^{(2, q)}$ does not depend on $q$. The proof is quite similar to that of point 1) of Theorem 1.1.2. It hinges upon :

- $\varphi_{q}(z)>0$ for every $q \in \mathcal{I}$ and $z \in \mathbb{C}$;

- $\frac{\varphi_{q_{1}}(z)}{\varphi_{q_{2}}(z)} \underset{|z| \rightarrow \infty}{\longrightarrow} 1$ for every $q_{1}$ and $q_{2} \in \mathcal{I}$;

- $\varphi_{q}(z) \underset{|z| \rightarrow \infty}{\longrightarrow}+\infty$ and the $\left(W_{z, \infty}^{(2, q)}, z \in \mathbb{C}\right)$ process $\left(X_{t}, t \geq 0\right)$ is transient.

These properties follow from Theorem 2.1.1. We also note, just as we did in Lemma 1.1.3 :

$$
\begin{aligned}
& W_{z, \infty}^{(2, q)}\left(\exp +\frac{\lambda}{2} A_{\infty}^{(q)}\right)<\infty \text { if } \lambda<1 \\
& W_{z, \infty}^{(2, q)}\left(\exp +\frac{\lambda}{2} A_{\infty}^{(q)}\right)=\infty \text { if } \lambda \geq 1
\end{aligned}
$$

These two properties show that $\mathbf{W}^{(2)}$ is well defined via (2.1.10) (since $A_{\infty}^{(q)}<\infty W_{\infty}^{(2, q)}$ a.s.) and that $\mathbf{W}^{(2)}$ has infinite total mass ; it is $\sigma$-finite on $\left(\Omega, \mathcal{F}_{\infty}\right)$ and it is such that $\mathbf{W}^{(2)}\left(\Gamma_{t}\right)=0$ or $+\infty$ for any $\Gamma_{t} \in b^{+}\left(\mathcal{F}_{t}\right)$ depending whether $W^{(2)}\left(\Gamma_{t}\right)$ is equal to 0 or is strictly positive. 


\subsection{Properties of $\mathrm{W}^{(2)}$.}

2.2.1 Some notation.

We shall now present Theorem 2.2.1 - which plays for $\mathbf{W}^{(2)}$ a similar role to that of Theorem 1.1.5 for $\mathbf{W}$. However, in order to state Theorem 2.2.1, we need the following notation :

i) Denote by $C$ the unit circle in $\mathbb{C}$ :

$$
C=\{z \in \mathbb{C} ;|z|=1\}
$$

and $\left(L_{t}^{(C)}, t \geq 0\right)$ the (continuous) local time process on $C$, which may be defined as :

$$
L_{t}^{(C)}:=\lim _{\varepsilon \downarrow 0} \frac{1}{2 \pi \varepsilon} \int_{0}^{t} 1_{C_{\varepsilon}}\left(X_{s}\right) d s
$$

where

$$
C_{\varepsilon}=\{z \in \mathbb{C} ; 1-\varepsilon \leq|z| \leq 1+\varepsilon\}
$$

so that, a.s. if $q_{0}$ denotes the uniform probability on $C$ :

$$
\int_{C} f(z) q_{0}(d z)=\frac{1}{2 \pi} \int_{0}^{2 \pi} f\left(e^{i \theta}\right) d \theta
$$

we have :

$$
\left(L_{t}^{(C)}, t \geq 0\right)=\left(A_{t}^{\left(q_{0}\right)}, t \geq 0\right)
$$

We denote by $\left(\tau_{l}^{(C)}, l \geq 0\right)$ the right continuous inverse of $\left(L_{t}^{(C)}, t \geq 0\right)$ :

$$
\tau_{l}^{(C)}:=\inf \left\{t \geq 0 ; L_{t}^{(C)}>l\right\}, \quad l \geq 0
$$

and we denote by $W^{\left(2, \tau_{l}^{(C)}\right)}$ the law of a 2-dimensional Brownian motion starting from 0 , considered up to $\tau_{l}^{(C)}$.

ii) We denote by $P_{1}^{(2, \log )}$ the law of the process $\left(R_{t}, t \geq 0\right)$ which solves the stochastic differential equation :

$$
R_{t}=1+\beta_{t}+\int_{0}^{t} \frac{d s}{R_{s}}\left(\frac{1}{2}+\frac{1}{\log R_{s}}\right)
$$

where $\left(\beta_{t}, t \geq 0\right)$ is a 1 -dimensional Brownian motion starting from 0 . We note that the process $\left(R_{t}, t \geq 0\right)$ starts from 1 and that $P\left\{R_{t}>1\right.$ for every $\left.t>0\right\}=1$.

We adopted the notation $P_{1}^{(2, \log )}$ to indicate :

a) that this process starts from 1 ;

b) that it "differs at infinity from a 2-dimensional Bessel process" by the presence of the term $\frac{1}{\log R_{s}}$.

iii) $\underline{\text { Here is another description of the process }}\left(R_{t}, t \geq 0\right)$ defined by $(2.2 .6)$ :

$$
\left(\log R_{t}, t \geq 0\right) \stackrel{(\text { law })}{=}\left(\rho_{H_{t}}, t \geq 0\right)
$$

with :

- $\left(\rho_{u}, u \geq 0\right)$ a 3 -dimensional Bessel process starting from 0 ; 
- $H_{t}:=\int_{0}^{t} \frac{d s}{R_{s}^{2}}$

We prove (2.2.7).

We apply Itô's formula to the process $\left(R_{t}\right)$ solution of $(2.2 .6)$ and we obtain :

$$
\log R_{t}=\int_{0}^{t} \frac{d \beta_{s}}{R_{s}}+\int_{0}^{t} \frac{d s}{R_{s}^{2} \cdot \log R_{s}}
$$

We denote by $\left(\nu_{h}, h \geq 0\right)$ the inverse of the process $\left(H_{t}, t \geq 0\right)$ and we replace $t$ by $\nu_{h}$ in (2.2.9). Thus :

$$
\begin{aligned}
\log R_{\nu_{h}} & =\int_{0}^{\nu_{h}} \frac{d \beta_{s}}{R_{s}}+\int_{0}^{\nu_{h}} \frac{d s}{R_{s}^{2} \log R_{s}} \\
& =\widetilde{\beta}_{h}+\int_{0}^{h} \frac{d u}{\log R_{\nu_{u}}}
\end{aligned}
$$

after the change of variable $s=\nu_{u}$ and with $\left(\widetilde{\beta}_{h}, h \geq 0\right):=\left(\int_{0}^{\nu_{h}} \frac{d \beta_{s}}{R_{s}^{2}}, h \geq 0\right)$, which is a 1-dimensional Brownian motion since this - local - martingale admits as bracket $\left(\int_{0}^{\nu_{h}} \frac{d s}{R_{s}^{2}}=\right.$ $\left.H_{\nu_{h}}=h, h \geq 0\right)$. Hence, from (2.2.14) $\left(\log R_{\nu_{h}}, h \geq 0\right)$ is a 3 -dimensional Bessel process starting from 0 .

iv) Let now $\left(\alpha_{t}, t \geq 0\right)$ be another 1-dimensional Brownian motion, independent from $\left(\beta_{t}, t \geq 0\right)$ (hence independent from $\left(R_{t}, t \geq 0\right)$ ). We define the law $W^{\left(2, \tau_{l}^{(C)}\right)} \circ \widetilde{P}_{1}^{(2, \log )}$ as the law of the 2-dimensional process $\left(Y_{t}, t \geq 0\right)$ satisfying to :

a) $\left(Y_{t}, t \leq \tau_{l}^{(C)}\right)$ is a 2-dimensional Brownian motion starting from 0 and stopped in $\tau_{l}^{(C)}$; its law, from point $i$ ), is $W^{\left(2, \tau_{l}^{(C)}\right)}$

b) after $\tau_{l}^{(C)}$, the process $\left(Y_{\tau_{l}^{(C)}+t} t \geq 0\right)$ writes :

$$
Y_{\tau_{l}^{(C)}+t}:=R_{t} \cdot e^{i \alpha_{H_{t}}} \quad(t \geq 0)
$$

where :

- the law of the process $\left(R_{t}, t \geq 0\right)$ is $P_{1}^{(2, \log )}$

- $\left(\alpha_{t}, t \geq 0\right)$ is a 1-dimensional Brownian motion starting from $\alpha_{0}$, with $e^{i \alpha_{0}}=Y_{\tau_{l}^{(C)}}$ (we note that $\left.Y_{\tau_{l}^{(C)}} \in C\right)$

- $H_{t}=\int_{0}^{t} \frac{d s}{R_{s}^{2}}$

c) $\left(\alpha_{t}, t \geq 0\right)$ and $\left(\beta_{t}, t \geq 0\right)$ (the driving Brownian of $\left(R_{t}, t \geq 0\right)$, see (2.2.6)) are, conditionally on $\alpha_{0}$ independent from the process $\left(Y_{t}, t \leq \tau_{l}^{(C)}\right)$.

Formula (2.2.7) - the second description of $\left(R_{t}, t \geq 0\right)$ - permits to write (2.2.12) in another form :

$$
Y_{\tau_{l}^{(C)}+t}=\left.\exp \left(\rho_{u}+i \alpha_{u}\right)\right|_{u=H_{t}} \quad(t \geq 0)
$$


where $\left(\rho_{u}, u \geq 0\right)$ is a 3-dimensional Bessel process starting from 0 and $H_{t}=\int_{0}^{t} \frac{d s}{R_{s}^{2}}$.

2.2.2 Description of the canonical process $\left(X_{t}, t \geq 0\right)$ under $W_{\infty}^{\left(2, q_{0}\right)}$.

In order to describe the measure $\mathbf{W}^{(2)}$, we shall use the formula :

$$
\mathbf{W}^{(2)}=\varphi_{q_{0}}(0)\left(e^{\frac{1}{2} L_{\infty}^{(C)}}\right) \cdot W_{\infty}^{\left(2, q_{0}\right)}
$$

This is formula (2.1.10), with $q=q_{0}$ (in fact, we use here a slight extension of (2.1.10) since $q_{0}$ is not absolutely continuous with respect to Lebesgue measure on $\mathbb{C}$ ). We now need to study the probability $W_{\infty}^{\left(2, q_{0}\right)}$. This is the aim of the following Theorem :

Theorem 2.2.1. With the notation of Theorem 2.1.1:

1) $\varphi_{q_{0}}(z)=2+\frac{1}{\pi} \log |z| \quad$ if $|z| \geq 1$

$$
=2 \quad \text { if }|z| \leq 1
$$

and $\left(M_{s}^{q_{0}}, s \geq 0\right)$ is the martingale defined by:

$$
\begin{aligned}
M_{s}^{\left(q_{0}\right)} & =\frac{\varphi_{q_{0}}\left(X_{s}\right)}{\varphi_{q_{0}}(0)} \exp \left(-\frac{1}{2} L_{s}^{(C)}\right) \\
& =1+\frac{1}{\varphi_{q}(0)} \int_{0}^{s}<\nabla \varphi_{q_{0}}\left(X_{u}\right), d X_{u}>e^{-\frac{1}{2} L_{u}^{(C)}}
\end{aligned}
$$

2) Let $g_{C}:=\sup \left\{t \geq 0 ; X_{t} \in C\right\}$. Then $g_{C}$ is $W_{\infty}^{\left(2, q_{0}\right)}$ a.s. finite and the r.v. $L_{\infty}^{(C)}\left(=L_{g_{C}}^{(C)}\right)$ admits as density $f_{L_{\infty}^{(C)}}^{\left(2, q_{0}\right)}$ with :

$$
f_{L_{\infty}^{(C)}}^{W_{\infty}^{\left(2, q_{0}\right)}}(l)=\frac{1}{2} e^{-\frac{l}{2}} 1_{[0, \infty[}(l)
$$

3) Under the probability $W_{\infty}^{\left(2, q_{0}\right)}$ :

i) $\left(X_{s}, s \leq g_{C}\right)$ and $\left(X_{g_{C}+s}, s \geq 0\right)$ are independent

ii) The law of the process $\left(X_{g_{C}+s}, s \geq 0\right)$ is $\widetilde{P}_{1}^{(2, \log )}$ (defined in point 2.2.1, iv))

iii) Conditionally on $L_{g_{C}}^{(C)}=l$ the process $\left(X_{s}, s \leq g_{C}\right)$ is a 2-dimensional Brownian process stopped at $\tau_{l}^{(C)}$, and its law, from point 2.2.1 i), is $W_{0}^{\left(2, \tau_{l}^{(C)}\right)}$.

In other terms :

iv) $\quad W_{\infty}^{\left(2, q_{0}\right)}=\frac{1}{2} \int_{0}^{\infty} e^{-\frac{l}{2}} d l\left(W_{0}^{\left(2, \tau_{l}^{(C)}\right)} \circ \widetilde{P}_{1}^{(2, \log )}\right)$

We note, in particular, that $X_{\tau_{l}^{(C)}}$ under $W_{\infty}^{\left(2, q_{0}\right)}$ is uniformly distributed on $C$.

\section{Proof of Theorem 2.2.1.}

In dimension 1, this Theorem is, essentially, proven in ([RVY, II $])$. The only item which really differs from those of Theorem 8 in [RVY, II] is point 3 , ii). We shall emphasize the corresponding arguments.

We prove point 3, ii).

We first recall and adapt to dimension 2 the notation and results of [RVY, II]. 
i) Let $\left(\mathcal{G}_{t}, t \geq 0\right)$ be the smallest filtration containing $\left(\mathcal{F}_{t}, t \geq 0\right)$ and such that $g_{C}$ is a $\left(\mathcal{G}_{t}, t \geq 0\right)$ stopping time. Then, there exists a $\left(\left(\mathcal{G}_{t}, t \geq 0\right), W_{\infty}^{2, q_{0}}\right) 2$-dimensional Brownian motion $\left(B_{t}, t \geq 0\right)$ such that :

$$
X_{t}=B_{t}+\int_{t \wedge g_{C}}^{t} \frac{n_{u}}{M_{u}^{\left(q_{0}\right)}-\underline{M}_{u}^{\left(q_{0}\right)}} d u
$$

with :

- $n_{u}:=e^{-\frac{1}{2} L_{u}^{(C)}} \cdot \frac{\nabla \varphi_{q_{0}}\left(X_{u}\right)}{\varphi_{q_{0}}(0)}$

- $M_{u}^{\left(q_{0}\right)}$ is defined by $(2.2 .16)$ and :

$$
\underline{M}_{u}^{\left(q_{0}\right)}:=\inf _{s \leq u} M_{s}^{\left(q_{0}\right)}
$$

ii) The function $\underline{\varphi_{q_{0}}(z)}=2+\frac{1}{\pi} \log |z|$ (for $|z| \geq 1$ ) (see (2.2.15)) is increasing in $|z|$. On the other hand, for $u \geq g_{C}, L_{u}^{\left(q_{0}\right)}=L_{g_{C}}^{(C)}$. Thus :

$$
\begin{aligned}
\underline{M}_{u}^{\left(q_{0}\right)} & =M_{g_{C}}^{\left(q_{0}\right)}=\frac{\varphi_{q_{0}}\left(X_{g_{C}}\right)}{\varphi_{q_{0}}(0)} e^{-\frac{1}{2} L_{g_{C}}^{C}} \\
& =e^{-\frac{1}{2} L_{g_{C}}^{(C)}}
\end{aligned}
$$

(from (2.2.15) and since $X_{g_{C}} \in C$ ).

iii) Gathering (2.2.20), (2.2.21) and (2.2.23), we obtain :

$$
\begin{aligned}
X_{t} & =B_{t}+\int_{t \wedge g_{C}}^{t} d u \frac{\nabla \varphi_{q_{0}}\left(X_{u}\right) e^{-\frac{1}{2} L_{g_{C}}^{(C)}}}{\varphi_{q_{0}}\left(X_{u}\right) e^{-\frac{1}{2} L_{g_{C}}^{(C)}}-2 e^{-\frac{1}{2} L_{g_{C}}^{(C)}}}, \\
& \left.=B_{t}+\int_{t \wedge g_{C}}^{t} \frac{\nabla(\log |\cdot|)\left(X_{u}\right)}{\log \left|X_{u}\right|} d u \text { (after simplification by } e^{-\frac{1}{2} L_{g_{C}}^{(C)}}\right)
\end{aligned}
$$

(from (2.2.15), since $\varphi_{q_{0}}\left(X_{u}\right)-2=\frac{1}{\pi} \log \left|X_{u}\right|$ and $\left.\nabla \varphi_{q_{0}}\left(X_{u}\right)=\frac{1}{\pi}(\nabla \log |\cdot|)\left(X_{u}\right)\right)$.

iv) We now use Itô's formula to express $\left|X_{g_{C}+t}\right|:=\widetilde{R}_{t}$. We obtain, from (2.2.24) :

$$
\widetilde{R}_{t}=\left(\widetilde{B}_{g_{C}+t}-\widetilde{B}_{g_{C}}\right)+\int_{0}^{t} \frac{d s}{\widetilde{R}_{s}}\left(\frac{1}{2}+\frac{1}{\log \widetilde{R}_{s}}\right)
$$

where $\left(\widetilde{B}_{g_{C}+t}-\widetilde{B}_{g_{C}}, t \geq 0\right)$ is a 1-dimensional Brownian motion started at 1 . Thus, from (2.2.6), the law of $\left(\left|X_{g_{C}+t}\right|, t \geq 0\right)$ is $P_{1}^{(2, \log )}$.

Now, operating in an analoguous manner to calculate $\operatorname{Arg}\left(X_{g_{C}+t}\right)$, we obtain :

$$
\left(X_{g_{C}+t}, t \geq 0\right)=\left(R_{t} e^{i \alpha_{H_{t}}}, t \geq 0\right)
$$

with notation of points 2.2.1, ii), iii) and $i v)$.

2.2.3 Another description of the measure $\mathbf{W}^{(2)}$.

We now present a description of $\mathbf{W}^{(2)}$ which is analogous, in dimension 2 , to the description of $\mathbf{W}$ given by Theorem 1.1.8. 


\section{Theorem 2.2.2.}

1) $\mathbf{W}^{(2)}=\int_{0}^{\infty} d l\left(W_{0}^{\left(2, \tau_{l}^{(C)}\right)} \circ \widetilde{P}_{1}^{(2, \log )}\right)$

2) For every $t \geq 0$ and $\Gamma_{t} \in b\left(\mathcal{F}_{t}\right)$ :

$$
\mathbf{W}^{(2)}\left[\Gamma_{t} 1_{g_{C} \leq t}\right]=\frac{1}{\pi} W^{(2)}\left[\Gamma_{t} \log ^{+}\left(\left|X_{t}\right|\right)\right]
$$

(Recall that $\left.g_{C}:=\sup \left\{s \geq 0 ; X_{s} \in C\right\}\right)$

3) $i) \quad \mathbf{W}^{(2)}\left(g_{C} \in d t\right)=e^{-\frac{1}{2 t}} \frac{d t}{2 \pi t} \quad(t \geq 0)$

ii) Conditionally on $g_{C}=t$, the law of the process $\left(X_{u}, u \leq g_{C}\right)$, under $\mathbf{W}^{(2)}$ is $\Pi_{0}^{(2, t, U)}$, where:

- $U$ is a r.v. uniformly distributed on $C$;

- Conditionally on $U=u, \Pi_{0}^{(2, t, U)}$ is the law of a 2-dimensional Brownian bridge $\left(b_{s}^{(2, t, u)}, 0 \leq s \leq t\right)$ of length $t$ such that $b_{0}^{(2, t, u)}=0$ and $b_{t}^{(2, t, u)}=u$.

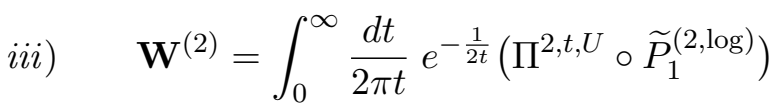

\section{Proof of Theorem 2.2.2.}

i) Point 1) is an easy consequence of (2.2.14), (2.2.19) and (2.2.18).

ii) We now show (2.2.27)

For this purpose, we use the definition (2.1.10) of $\mathbf{W}^{(2)}$ with $q=\lambda q_{0}$ (where $q_{0}$ is defined by (2.2.3), and $\lambda>0)$. We have :

$$
\varphi_{\lambda q_{0}}(z)=\frac{2}{\lambda}+\frac{1}{\pi} \log ^{+}(|z|)
$$

(see $(2.2 .15))$. Thus, for every $t \geq 0$ and $\Gamma_{t} \in b\left(\mathcal{F}_{t}\right)$ :

$$
\begin{aligned}
W^{(2)}\left(\Gamma_{t}\left(\frac{2}{\lambda}+\frac{1}{\pi} \log ^{+}\left(\left|X_{t}\right|\right)\right)\right) & =\varphi_{\lambda q_{0}}(0) W_{\infty}^{\left(2, \lambda q_{0}\right)}\left(\Gamma_{t} e^{\left.-\frac{\lambda}{2} L_{t}^{(C)}\right)}\right) \\
& =\mathbf{W}^{(2)}\left(\Gamma_{t} e^{-\frac{\lambda}{2}\left(L_{\infty}^{(C)}-L_{t}^{(C)}\right.}\right)
\end{aligned}
$$

We then let $\lambda \rightarrow \infty$ in $(2.2 .32)$ and note that $L_{\infty}^{(C)}-L_{t}^{(C)}>0$ on the set $\left(g_{C}>t\right)$ (and equals to 0 on $g_{C} \leq t$ ). The monotone convergence Theorem implies :

$$
\frac{1}{\pi} W^{(2)}\left(\Gamma_{t} \log ^{+}\left(\left|X_{t}\right|\right)=\mathbf{W}^{(2)}\left(\Gamma_{t} 1_{g_{C} \leq t}\right)\right.
$$

This is (2.2.28). Note that we may replace $t$ by a stopping time $T$ in (2.2.28). We obtain :

$$
\mathbf{W}^{(2)}\left(\Gamma_{T} 1_{g_{C} \leq T<\infty}\right)=\frac{1}{\pi} W^{(2)}\left(\Gamma_{T} \log ^{+}\left(\left|X_{T}\right|\right) 1_{T<\infty}\right)
$$

with $\Gamma_{T} \in b\left(\mathcal{F}_{T}\right)$. 


\section{Remark 2.2.3.}

We deduce from (2.2.32) and (2.2.28) :

$$
\begin{aligned}
\frac{2}{\lambda} W^{(2)}\left(\Gamma_{t}\right) & =\mathbf{W}^{(2)}\left(\Gamma_{t} 1_{g_{C}>t} \exp \left(-\frac{\lambda}{2}\left(L_{\infty}^{(C)}-L_{t}^{(C)}\right)\right)\right. \\
& =W^{(2)}\left(\Gamma_{t}\right)\left(\int_{0}^{\infty} e^{-\frac{\lambda}{2} l} d l\right)
\end{aligned}
$$

and

$$
W^{(2)}\left(\log ^{+}\left|X_{t}\right|\right)=\mathbf{W}^{(2)}\left(g_{C} \leq t\right)=\mathbf{W}^{(2)}\left(L_{\infty}^{(C)}-L_{t}^{(C)}=0\right)
$$

Then, operating as in the proof of Theorem 1.1.6, point 3) i) (see (1.1.45) and (1.1.46)), we obtain :

i) $\mathbf{W}^{(2)}\left(L_{\infty}^{(C)}-L_{t}^{(C)} \in d l\right)=1_{[0, \infty[}(l) d l+W^{(2)}\left(\log ^{+}\left(\left|X_{t}\right|\right)\right) \delta_{0}(d l)$

ii) Conditionally on $L_{\infty}^{C}-L_{t}^{C}=l \quad(l>0),\left(X_{u}, u \leq t\right)$ is, under $\mathbf{W}^{(2)}$, a 2-dimensional Brownian motion indexed by $[0, t]$.

Remark 2.2.4. We can obtain (2.2.28) in the same manner as for point 2) of Remark 1.2.9. For this purpose, we need a scale function for the $W^{\left(2, q_{0}\right)}$ process. The function $z \rightarrow \frac{1}{1+\frac{1}{\pi} \log (|z|)}(|z| \geq 1)$ is an adequate choice.

iii) We now prove point 3 i) of Theorem 2.2.2.

We write (2.2.28) with $\Gamma_{t} \equiv 1$ :

$$
\mathbf{W}^{(2)}\left(g_{C} \leq t\right)=\frac{1}{\pi} W^{(2)}\left(\log ^{+}\left|X_{t}\right|\right)
$$

and we differentiate (2.2.37) with respect to $t$. Thus :

$$
\begin{aligned}
\mathbf{W}^{(2)}\left(g_{C} \in d t\right) & =\frac{1}{\pi}\left(\frac{d}{d t} W^{(2)}\left(\log ^{+}\left|X_{t}\right|\right)\right) \cdot d t \\
& =\frac{1}{\pi} \frac{d}{d t} W^{(2)}\left(1_{\left|X_{1}\right|>\frac{1}{\sqrt{t}}}\left(\log \sqrt{t}-\log \frac{1}{\left|X_{1}\right|}\right)\right) \cdot d t \\
\text { (by scaling) } & =\frac{1}{2 \pi t} W^{(2)}\left(\frac{\left|X_{1}\right|^{2}}{2}>\frac{1}{2 t}\right) d t \\
& =\frac{1}{2 \pi t} e^{-\frac{1}{2 t}} d t \quad(t \geq 0)
\end{aligned}
$$

since $\frac{\left|X_{1}\right|^{2}}{2}$ is a standard exponential r.v.

The end of the proof of Theorem 2.2.2 is obtained by using arguments similar to those used for Theorem 1.1.6. We note, in particular, that conditionally on $X_{g_{C}},\left(X_{g_{C}+t}, t \geq 0\right)$ and $\left(X_{s}, s \leq g_{C}\right)$ are independent.

Remark 2.2.5. From (2.2.29), we deduce :

$$
\mathbf{W}^{(2)}\left(e^{-\frac{\lambda^{2}}{2} g_{C}}\right)=\int_{0}^{\infty} \frac{d t}{2 \pi t} e^{-\frac{\lambda^{2}}{2} t-\frac{1}{2 t}}=K_{0}(\lambda)
$$

where $K_{0}$ denotes the Bessel-Mc Donald function with index 0 (see [Leb], formula 5.10.25). 


\subsection{Study of the winding process under $\mathbf{W}^{(2)}$.}

Formula (2.2.12) :

$$
X_{g_{C}+t}=R_{t} e^{i \alpha_{H_{t}}},(t \geq 0)
$$

which provider a representation after $g_{C}$ under $W^{(2)}$ invites to establish for this process a theorem similar to the classical theorem of Spitzer, which we recall :

2.3.1 Spitzer's Theorem.

Theorem. (Spitzer $[\mathrm{S}]$ )

Let $\left(X_{t}, t \geq 0\right)$ a 2-dimensional Brownian motion, $\mathbb{C}$ valued, starting from $z \neq 0$. We have :

$$
X_{t}=\left|X_{t}\right| e^{i \alpha_{H_{t}}}
$$

with :

i) $\left(\alpha_{u}, u \geq 0\right)$ a 1-dimensional Brownian motion independent from the 2-dimensional Bessel process $\left(\left|X_{t}\right|, t \geq 0\right)$.

ii) $\quad H_{t}=\int_{0}^{t} \frac{d s}{\left|X_{s}\right|^{2}}$

Let $\left(\theta_{t}, t \geq 0\right):=\left(\alpha_{H_{t}}, t \geq 0\right)=\left(\theta_{0}+\operatorname{Im} \int_{0}^{t} \frac{d X_{s}}{X_{s}}, t \geq 0\right)$ be the winding process. Then :

$$
\frac{2 \theta_{t}}{\log t} \underset{t \rightarrow \infty}{\stackrel{(\text { law })}{\longrightarrow}} \Gamma \stackrel{(\text { law })}{=} \alpha_{T_{1}(\gamma)}
$$

iii) $\left(\gamma_{t}, t \geq 0\right)$ is a 1-dimensional Brownian motion started from 0 and independent from $\left(\alpha_{u}, u \geq 0\right)$. and :

$$
T_{1}(\gamma):=\inf \left\{s \geq 0 ; \gamma_{s}=1\right\}
$$

iv) Consequently $\Gamma$ is a standard Cauchy r.v.

2.3.2. An analogue of Spitzer's Theorem.

Now, here is the analogue of the above (Spitzer) Theorem for the process $\left(X_{g_{C}+t}, t \geq 0\right)$ :

Theorem 2.3.1. Under $\widetilde{P}_{1}^{(2, \log )}$, the winding process $\left(\theta_{t}, t \geq 0\right)=\left(\alpha_{H_{t}}, t \geq 0\right)$ satisfies :

1) $\frac{4}{(\log t)^{2}} H_{t} \underset{t \rightarrow \infty}{\stackrel{(\text { law })}{\longrightarrow}} T_{1}^{(3)}$

where $T_{1}^{(3)}:=\inf \left\{u ; \rho_{u}=1\right\}$

is the first hitting time of level 1 by a 3-dimensional Bessel process $\left(\rho_{u}, u \geq 0\right)$ started at 0.

2) $\frac{2}{\log t} \theta_{t} \stackrel{(\text { law })}{\longrightarrow} \alpha_{T_{1}^{(3)}}$

where $\left(\alpha_{u}, u \geq 0\right)$ is a 1-dimensional Brownian motion independent from $\left(\rho_{u}, u \geq 0\right)$.

We now recall our notation (see Section 2.2.1)

- $\left(R_{t}, t \geq 0\right)$ is the process defined in $(2.2 .6)$

- $H_{t}=\int_{0}^{t} \frac{d s}{R_{s}^{2}}$

- $\left(\alpha_{u}, u \geq 0\right)$ is a 1-dimensional Brownian motion independent from $\left(R_{t}, t \geq 0\right)$

- $\left(\log R_{t}, t \geq 0\right)=\left(\rho_{H_{t}}, t \geq 0\right)$ and $\left(\rho_{u}, u \geq 0\right)$ is a 3 -dimensional Bessel process started at 0 . 


\section{Remark 2.3.2.}

1. Theorem 2.3.1 differs from Spitzer's Theorem in that $T_{1}$ has been replaced by $T_{1}^{(3)}$.

2. Let, for every $z \in \mathbb{C}, \mathbf{W}_{z}^{(2)}$ be defined by :

$$
\mathbf{W}_{z}^{(2)}\left(F\left(X_{s}, s \geq 0\right)\right):=\mathbf{W}^{(2)}\left(F\left(z+X_{s}, s \geq 0\right)\right)
$$

Theorem 2.3.1 then implies that, for $z \neq 0$, under $\mathbf{W}_{z}^{(2)}$ and conditionally on $g_{C} \leq a$, the winding process $\left(\theta_{t}, t \geq 0\right)$ satisfies :

$$
\frac{2}{\log t} \theta_{t} \underset{t \rightarrow \infty}{\longrightarrow} \alpha_{T_{1}^{(3)}}
$$

for all $a>0$. This easily results from (2.3.7) and from the representation formula (2.2.6).

Proof of Theorem 2.3.1.

i) We use the notation (2.3.8). We admit for a moment that :

$$
\begin{gathered}
H_{t}-H_{T_{\sqrt{t}}(R)} \quad \text { converges in law as } t \rightarrow \infty, \text { with : } \\
T_{\sqrt{t}(R)}:=\inf \left\{s \geq 0 ; R_{s} \geq \sqrt{t}\right\}
\end{gathered}
$$

and we show that (2.3.10) implies Theorem 2.3.1. Indeed, from (2.3.10), we have :

$$
\frac{4}{(\log t)^{2}} H_{t} \underset{t \rightarrow \infty}{\sim} \frac{1}{(\log \sqrt{t})^{2}} H_{T_{\sqrt{t}}(R)}
$$

But :

$$
\frac{1}{(\log a)^{2}} H_{T_{a}(R)}=\frac{1}{(\log a)^{2}} T_{\log a}(\rho) \stackrel{(\text { law })}{=} T_{1}(\rho)
$$

with

$$
T_{\log (a)}(\rho):=\inf \left\{t \geq 0 ; \rho_{t} \geq \log a\right\}
$$

The first equality in (2.3.13) results from definitions (see (2.3.8) and (2.3.9)) and the second from the scaling property. Thus, from (2.3.10), we deduce :

$$
\frac{4}{(\log t)^{2}} H_{t} \underset{t \rightarrow \infty}{\stackrel{(\text { law })}{\longrightarrow}} T_{1}^{(3)}
$$

and

$$
\begin{aligned}
\frac{2}{\log t} \theta_{t}= & \frac{2}{\log t} \alpha_{H_{t}} \stackrel{(\text { law })}{=} \frac{2 \sqrt{H_{t}}}{\log t} \alpha_{1} \quad \text { (by scaling) } \\
& \stackrel{(\text { law })}{\stackrel{ }{w} \sqrt{T_{1}^{(3)}} \cdot \alpha_{1}} \\
& \stackrel{(\text { law })}{=} \alpha_{T_{1}^{(3)}} \quad \text { (by scaling) }
\end{aligned}
$$

which proves Theorem 2.3.1.

ii) It remains to prove (2.3.10).

For this purpose, we start with the following Lemma :

Lemma 2.3.3. Let $\left(R_{t}, t \geq 0\right)$ be defined by (2.2.6). Then : $\left(\frac{1}{\sqrt{t}} R_{t v}, v \geq 0\right)$ converges in law, as $t \rightarrow \infty$, to a 2-dimensional Bessel process starting from 0 . 


\section{Proof of Lemma 2.3.3.}

From (2.2.6) we have :

$$
R_{t}=1+\beta_{t}+\int_{0}^{t}\left(\frac{1}{2 R_{s}}+\frac{1}{R_{s} \log R_{s}}\right) d s
$$

Thus :

$$
\frac{1}{\sqrt{t}} R_{t v}=\frac{1}{\sqrt{t}}+\frac{1}{\sqrt{t}} \beta_{t v}+\frac{1}{\sqrt{t}} \int_{0}^{t v}\left(\frac{1}{2 R_{s}}+\frac{1}{R_{s} \log R_{s}}\right) d s
$$

Denoting by $\left(\widetilde{\beta}_{v}, v \geq 0\right)$ the Brownian motion $\left(\frac{1}{\sqrt{t}} \beta_{t v}, v \geq 0\right)$ and making the change of variable $s=t v$, we obtain, with $\left(\widetilde{R}_{v}^{(t)}=\frac{1}{\sqrt{t}} R_{t v}, v \geq 0\right)$ :

$$
\widetilde{R}_{v}^{(t)}=\frac{1}{\sqrt{t}}+\widetilde{\beta}_{v}+\int_{0}^{u}\left(\frac{1}{2 \widetilde{R}_{u}^{(t)}}+\frac{1}{\widetilde{R}_{u}^{(t)}\left(\log \sqrt{t}+\log \widetilde{R}_{u}^{(t)}\right)}\right) d u
$$

Hence, as $t \rightarrow \infty,\left(\widetilde{R}_{v}^{(t)}, v \geq 0\right)$ converges in law to the law of the solution of the SDE.

$$
\widetilde{R}_{v}=\widetilde{\beta}_{v}+\int_{0}^{v} \frac{d u}{2 \widetilde{R}_{u}}
$$

i.e. to (the law of) a 2-dimensional Bessel process started at 0 .

iv) We may now end up the proof of (2.3.10).

We have, from (2.3.8) :

$$
H_{T_{\sqrt{t}}(R)}-H_{t}=\int_{t}^{T_{\sqrt{t}}(R)} \frac{d v}{R_{u}^{2}}=\int_{1}^{\frac{1}{t} T_{\sqrt{t}}(R)} \frac{d v}{\left(\frac{1}{t} R_{v t}^{2}\right)}
$$

after making the change of variable $u=t v$. But, from Lemma 2.3.3, $\left(\frac{1}{\sqrt{t}} R_{v t}, v \geq 0\right)$ converges in law to a 2-dimensional Bessel process starting from $0\left(R_{0}^{(2)}(v), v \geq 0\right)$. Thus : $H_{t}-H_{\sqrt{t}}(R)$ converges in law, as $t \rightarrow \infty$, to

$$
\int_{1}^{T_{1}\left(R_{0}^{(2)}\right)} \frac{d u}{\left(R_{0}^{(2)}(u)\right)^{2}}
$$

with $T_{1}\left(R_{0}^{(2)}\right)=\inf \left\{s \geq 0 ; R_{0}^{(2)}(s)=1\right\}$.

Remark 2.3.4. (An extension of Theorem 2.3.1.)

Let $\left(\beta_{t}, t \geq 0\right)$ denote a 1 -dimensional Brownian motion starting at $0, \delta>0$ and $\left(R_{t}^{(\delta)}\right.$, $t \geq 0)$ the solution of :

$$
R_{t}^{(\delta)}=1+\beta_{t}+\int_{0}^{t}\left(\frac{1}{2 R_{s}^{(\delta)}}+\frac{\delta}{R_{s}^{(\delta)} \log R_{s}^{(\delta)}}\right) d s
$$


The case we have just studied is that of $\delta=1$. Let :

$$
H_{t}^{(\delta)}:=\int_{0}^{t} \frac{d s}{\left(R_{s}^{(\delta)}\right)^{2}}
$$

and

$$
\theta_{t}^{(\delta)}=\alpha_{H_{t}^{(\delta)}}
$$

where $\left(\alpha_{u}, u \geq 0\right)$ is a 1 -dimensional Brownian motion independent from $\left(\beta_{t}, t \geq 0\right)$.

The technique we have just developed allows to obtain :

i) $\left(\log R_{t}^{(\delta)}, t \geq 0\right)=\left(\rho_{H_{t}^{(\delta)}}^{(2 \delta+1)}, t \geq 0\right)$

where $\left(\rho_{u}^{(2 \delta+1)}, u \geq 0\right)$ is a $(2 \delta+1)$-dimensional Bessel process starting at 0 .

ii) $\frac{4}{(\log t)^{2}} H_{t}^{(\delta)} \underset{t \rightarrow \infty}{\stackrel{(\text { law })}{\longrightarrow}} T_{1}^{(2 \delta+1)}$

where $T_{1}^{(2 \delta+1)}:=\inf \left\{u \geq 0 ; \rho_{u}^{(2 \delta+1)}=1\right\}$.

iii) $\frac{2 \theta_{t}^{(\delta)}}{\log t}=\frac{2 \alpha_{H_{t}}^{(\delta)}}{\log t} \underset{t \rightarrow \infty}{\stackrel{\text { law })}{\longrightarrow}} \alpha_{T_{1}^{(2 \delta+1)}}$

where $T_{1}^{(2 \delta+1)}$ is independent from the 1-dimensional Brownian motion $\left(\alpha_{u}, u \geq 0\right)$.

2.4 $W^{(2)}$ martingales associated to $\mathbf{W}^{(2)}$.

Just as in Chapter 1, we associated to any r.v. $F \in L^{1}\left(\mathcal{F}_{\infty}, \mathbf{W}\right)$ the $\left(\left(\mathcal{F}_{t}, t \geq 0\right), W\right)$ martingale $\left(M_{t}(F), t \geq 0\right)$, we now associate to every r.v. $F \in L^{1}\left(\mathcal{F}_{\infty}, \mathbf{W}^{(2)}\right)$ a $\left(\left(\mathcal{F}_{t}, t \geq 0\right), W^{(2)}\right)$ martingale $\left(M_{t}^{(2)}(F), t \geq 0\right)$.

2.4.1 Definition of $\left(M_{t}^{(2)}(F), t \geq 0\right)$.

Theorem 2.4.1. (Definition of $M_{t}^{(2)}(F), t \geq 0$ )

Let $F \in L^{1}\left(\Omega=\mathcal{C}\left(\mathbb{R} \rightarrow \mathbb{C}, \mathcal{F}_{\infty}, \mathbf{W}^{(2)}\right)\right.$. There exists a $\left(\left(\mathcal{F}_{t}, t \geq 0\right), W^{(2)}\right)$ martingale (which is necessarily continuous) $\left(M_{t}^{(2)}(F), t \geq 0\right)$, positive if $F \geq 0$, such that:

1) For every $t \geq 0$ and $\Gamma_{t} \in b\left(\mathcal{F}_{t}\right)$ :

$$
\mathbf{W}^{(2)}\left(F \cdot \Gamma_{t}\right)=W^{(2)}\left(M_{t}^{(2)}(F) \cdot \Gamma_{t}\right)
$$

In particular, for every $t \geq 0$ :

$$
\mathbf{W}^{(2)}(F)=W^{(2)}\left(M_{t}^{(2)}(F)\right)
$$

and, if $F$ and $G$ belong to $L_{+}^{1}\left(\mathcal{F}_{\infty}, \mathbf{W}^{(2)}\right)$ :

$$
W^{(2)}\left(M_{t}^{(2)}(F) \cdot M_{t}^{(2)}(G)\right)=\mathbf{W}^{(2)}\left(F \cdot M_{t}^{(2)}(G)\right)=\mathbf{W}^{(2)}\left(M_{t}^{(2)}(F) \cdot G\right)
$$

2) $M_{t}^{(2)}(F)=\widehat{W}_{X_{t}\left(\omega_{t}\right)}^{(2)}(F)\left(\omega_{t}, \widehat{\omega}^{t}\right)$

3) $M_{t}^{(2)}(F) \longrightarrow 0 \quad W^{(2)}$ a.s.

In particular, the martingale $\left(M_{t}^{(2)}(F), t \geq 0\right)$ is not uniformly integrable.

4) For every $q \in \mathcal{I}$ :

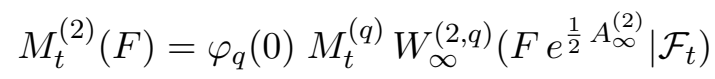


where $M_{t}^{(q)}, \varphi_{q}$ and $W_{\infty}^{(2, q)}$ are defined in Theorem 2.1.1.

The proof of Theorem 2.4.1 is, mutatis mutandis, the proof of Theorem 1.2.1. Here are some examples of martingales $\left(M_{t}^{(2)}(F), t \geq 0\right)$.

Example 2.1. Let $q \in \mathcal{I}$ and $F_{q}=\exp \left(-\frac{1}{2} A_{\infty}^{(q)}\right)$. We have, from (2.1.10) :

$$
\mathbf{W}^{(2)}\left(F_{q}\right)=\varphi_{q}(0)
$$

and

$$
\left(M_{t}^{(2)}\left(F_{q}\right)=\varphi_{q}\left(X_{t}\right) \exp \left(-\frac{1}{2} A_{t}^{(q)}\right), t \geq 0\right)
$$

In particular, for $q=\lambda q_{0} \quad($ see $(2.2 .3)$ and $(2.2 .31))$ :

$$
M_{t}^{(2)}\left(\exp -\frac{\lambda}{2} A_{\infty}^{\left(q_{0}\right)}\right)=\left(\frac{2}{\lambda}+\frac{1}{\pi} \log ^{+}\left(\left|X_{t}\right|\right)\right) \exp \left(-\frac{\lambda}{2} L_{t}^{(C)}\right)
$$

Example 2.2. (see [RVY, VI]).

We write the skew-product representation of the canonical 2-dimensional Brownian motion $\left(X_{t}, t \geq 0\right)$ starting at $z \neq 0$; as :

$$
X_{t}=\left|X_{t}\right| \cdot \exp \left(i \alpha_{H_{t}}\right)
$$

where :

i) $\left(\left|X_{t}\right|, t \geq 0\right)$ is a 2-dimensional Bessel process starting at $|z|$.

ii) $H_{t}=\int_{0}^{t} \frac{d s}{\left|X_{s}\right|^{2}}$

iii) $\left(\alpha_{u}, u \geq 0\right)$ is a 1 -dimensional Brownian process independent from $\left(\left|X_{u}\right|, u \geq 0\right)$.

Let $\left(\theta_{t}:=\alpha_{H_{t}}, t \geq 0\right)$ denote the winding process and introduce :

$$
S_{t}^{\theta}:=\sup _{s \leq t} \theta_{s}=\sup _{u \leq H_{t}} \alpha_{u}
$$

Let $\varphi: \mathbb{R}_{+} \rightarrow \mathbb{R}_{+}$Borel and integrable. Then :

$$
\left(M_{t}^{(2)}\left(\varphi\left(S_{\infty}^{\theta}\right), t \geq 0\right)=\left(\varphi\left(S_{t}^{\theta}\right)\left(S_{t}^{\theta}-\theta_{t}\right)+\int_{S_{t}^{\theta}}^{\infty} \varphi(y) d y, \quad t \geq 0\right)\right.
$$

2.4.2 A decomposition Theorem of positive $W^{(2)}$ supermartingales.

Just as in Theorem 1.2.5, we have obtained a decomposition Theorem for every $\left(\left(\mathcal{F}_{t}, t \geq 0\right)\right.$, $W)$ positive supermartingale, we now present a decomposition theorem for every $\left(\left(\mathcal{F}_{t}, t \geq 0\right)\right.$, $\left.W^{(2)}\right)$ positive supermartingale.

Theorem 2.4.2. Let $\left(Z_{t}, t \geq 0\right)$ denote a positive $\left(\Omega=\mathcal{C}\left(\mathbb{R}_{+}, \mathbb{C}\right),\left(\mathcal{F}_{t}, t \geq 0\right), W^{(2)}\right)$ supermartingale. We denote $Z_{\infty}:=\lim _{t \rightarrow \infty} Z_{t}, W^{(2)}$ a.s. Then :

1) $z_{\infty}:=\lim _{t \rightarrow \infty} \pi \frac{Z_{t}}{1+\log ^{+}\left(\left|X_{t}\right|\right)}$ exists $\mathbf{W}^{(2)}$ a.s.

and : $\quad \mathbf{W}^{(2)}\left(z_{\infty}\right)<\infty$ 
2) $\left(Z_{t}, t \geq 0\right)$ decomposes in a unique manner in the form:

$$
Z_{t}=M_{t}^{(2)}\left(z_{\infty}\right)+W^{(2)}\left(Z_{\infty} \mid \mathcal{F}_{t}\right)+\xi_{t} \quad(t \geq 0)
$$

where $\left(M_{t}^{(2)}\left(z_{\infty}\right), t \geq 0\right)$ and $\left(W^{(2)}\left(Z_{\infty} \mid \mathcal{F}_{t}\right), t \geq 0\right)$ denote two $\left(\left(\mathcal{F}_{t}, t \geq 0\right), W^{(2)}\right)$ martingales and:

$\left(\xi_{t}, t \geq 0\right)$ is a $\left(\left(\mathcal{F}_{t}, t \geq 0\right), W^{(2)}\right)$ positive martingale such that :

i) $Z_{\infty} \in L_{+}^{1}\left(\mathcal{F}_{\infty}, W^{(2)}\right)$, hence $W^{(2)}\left(Z_{\infty} \mid \mathcal{F}_{t}\right)$ converges $W^{(2)}$ a.s. and in $L^{1}\left(\mathcal{F}_{\infty}, W^{(2)}\right)$ towards $Z_{\infty}$

ii) $\quad \frac{W\left(Z_{\infty} \mid \mathcal{F}_{t}\right)+\xi_{t}}{1+\log ^{+}\left(\left|X_{t}\right|\right)} \underset{t \rightarrow \infty}{\longrightarrow} 0 \quad \mathbf{W}^{(2)}$ a.s.

iii) $\quad M_{t}^{(2)}\left(z_{\infty}\right)+\xi_{t} \underset{t \rightarrow \infty}{\longrightarrow} 0 \quad W^{(2)}$ a.s.

In particular, if $F \in L^{1}\left(\mathcal{F}_{\infty}, \mathbf{W}^{(2)}\right)$, then :

$$
\pi \cdot \frac{M_{t}(F)}{1+\log ^{+}\left(\left|X_{t}\right|\right)} \underset{t \rightarrow \infty}{\longrightarrow} F \quad \mathbf{W}^{(2)} \text { a.s. }
$$

and the map : $F \rightarrow\left(M_{t}^{(2)}(F), t \geq 0\right)$ is injective.

Corollary 2.4.3. (A characterisation of martingales of the form $\left(M_{t}^{(2)}(F), t \geq 0\right)$. A $\left(\left(\mathcal{F}_{t}, t \geq 0\right), W^{(2)}\right)$ positive martingale $\left(Z_{t}, t \geq 0\right)$ is equal to $\left(M_{t}^{(2)}(F), t \geq 0\right)$ for an $F \in L^{1}\left(\mathcal{F}_{\infty}, \mathbf{W}^{(2)}\right)$ if and only if :

$$
Z_{0}=\mathbf{W}^{(2)}\left(\lim _{t \rightarrow \infty} \pi \cdot \frac{Z_{t}}{1+\log ^{+}\left(\left|X_{t}\right|\right)}\right)
$$

Note that $\lim _{t \rightarrow \infty} \frac{Z_{t}}{1+\log ^{+}\left(\left|X_{t}\right|\right)}$ exists $\mathbf{W}^{(2)}$ a.s. from (2.4.13).

\section{Sketches of Proofs of Theorem 2.4.2 and of Corollary 2.4.3.}

This proof is essentially the same as those of Theorem 1.2.5 and of Corollary 1.2.6. Two arguments need to be modified :

i) The role of the r.v. $g$ in the proof of Theorem 1.2.5 is played here by that of the r.v. $g_{C}$.

ii) The relation (1.1.41): $\mathbf{W}\left(\Gamma_{t} 1_{g \leq t}\right)=W\left(\Gamma_{t}\left|X_{t}\right|\right)$

and the limiting result :

$$
\frac{\varphi_{q}\left(X_{t}\right) \exp \left(-\frac{1}{2} A_{t}^{(q)}\right)}{1+\left|X_{t}\right|} \underset{t \rightarrow \infty}{\longrightarrow} \exp \left(-\frac{1}{2} A_{\infty}^{(q)}\right)
$$

which were used in the proof of Lemma 1.2.8 need to be replaced respectively by :

$$
\left.\mathbf{W}^{(2)}\left(\Gamma_{t} 1_{\left(g_{C} \leq t\right)}\right)=\frac{1}{\pi} W^{(2)}\left(\Gamma_{t} \log ^{+}\left|X_{t}\right|\right)\right) .
$$

(This is relation (2.2.28) of Theorem 2.2.2) and by :

$$
\pi \cdot \frac{\varphi_{q}\left(X_{t}\right) \exp \left(-\frac{1}{2} A_{t}^{(q)}\right)}{1+\log ^{+}\left(\left|X_{t}\right|\right)} \underset{t \rightarrow \infty}{\longrightarrow} \exp \left(-\frac{1}{2} A_{\infty}^{(q)}\right) \quad \mathbf{W}^{(2)} \text { a.s. }
$$


The latter (2.4.19) follows easily from :

$$
\pi \cdot \varphi_{q}(z) \underset{|z| \rightarrow \infty}{\sim} \log (|z|), \quad \text { from }(2.1 .6)
$$

and from : $\left|X_{t}\right| \underset{t \rightarrow \infty}{\longrightarrow} \infty \quad \mathbf{W}^{(2)}$ a.s.

since the canonical process under $W_{\infty}^{(2, q)}$ is transient.

2.4.3 A decomposition Theorem for the martingales $\left(M_{t}^{(2)}(F), t \geq 0\right)$.

A difference with the preceding subsection is that the r.v.'s $F$ which we now consider belong to $L^{1}\left(\mathcal{F}_{\infty}, \mathbf{W}^{(2)}\right)$ but are not necessarily positive. Here is the analogue, in dimension 2 , of Theorem 1.2.11.

Theorem 2.4.4. $F \in L^{1}\left(\mathcal{F}_{\infty}, \mathbf{W}^{(2)}\right)$ and let $\left(M_{t}^{(2)}(F), t \geq 0\right)$ the $\left(\left(\mathcal{F}_{t}, t \geq 0\right), W^{(2)}\right)$ martingale associated to $F$ by Theorem 2.4.1. Let $C,\left(L_{t}^{(C)}, t \geq 0\right)$ and $g_{C}$ be as in Section 2.2.1, i) and Section 2.2.2. Then:

1) i) There exists a previsible process $\left(k_{s}^{(C)}(F), s \geq 0\right)$ which is defined $d L_{s}^{(C)} \cdot W^{(2)}(d \omega)$ a.s., positive if $F \geq 0$, and such that :

$$
W^{(2)}\left(\int_{0}^{\infty}\left|k_{s}^{(C)}(F)\right| d L_{s}^{(C)}\right)=\mathbf{W}^{(2)}\left(\left|k_{g_{C}}^{(C)}(F)\right|\right) \leq \mathbf{W}^{(2)}(|F|)<\infty
$$

and for every bounded previsible process $\left(\Phi_{s}, s \geq 0\right)$ :

$$
\begin{aligned}
\mathbf{W}^{(2)}\left(\Phi_{g_{C}} \cdot F\right) & =W^{(2)}\left(\int_{0}^{\infty} \Phi_{s} k_{s}^{(C)}(F) d L_{s}^{(C)}\right) \\
& =\mathbf{W}^{(2)}\left(\Phi_{g_{C}} k_{g_{C}}^{(C)}(F)\right)
\end{aligned}
$$

Thus :

$$
\mathbf{W}^{(2)}\left(F \mid \mathcal{F}_{g_{C}}\right)=k_{g_{C}}^{(C)}(F)
$$

ii) $\left(k_{s}^{(C)}\left(k_{g_{C}}^{(C)}(F), s \geq 0\right)=\left(k_{s}^{(C)}(F), s \geq 0\right)\right.$

iii) If $\left(h_{s}, s \geq 0\right)$ is a previsible process such that: $\mathbf{W}^{(2)}\left(\left|h_{g_{C}}\right|\right)<\infty$,

$$
\left(k_{s}^{(C)}\left(h_{g_{C}}\right), s \geq 0\right)=\left(h_{s}, s \geq 0\right) \quad d L_{s}^{(C)} \cdot W^{(2)}(d \omega) \text { a.s. }
$$

2) There exist two continuous quasimartingales $\left(\Sigma_{t}^{(2, C)}, t \geq 0\right)$ and $\left(\Delta_{t}^{(2, C)}, t \geq 0\right)$ such that, for every $t \geq 0$ :

$$
M_{t}^{(2)}(F)=\Sigma_{t}^{(2, C)}(F)+\Delta_{t}^{(2, C)}(F)
$$

with :

i) For every $t \geq 0$ and $\Gamma_{t} \in b\left(\mathcal{F}_{t}\right)$ :

$$
\begin{aligned}
& \mathbf{W}^{(2)}\left(\Gamma_{t} 1_{g_{C} \leq t} \cdot F\right)=W^{(2)}\left(\Gamma_{t} \Sigma_{t}^{(2, C)}(F)\right) \\
& \mathbf{W}^{(2)}\left(\Gamma_{t} 1_{g_{C} \leq t} \cdot F\right)=W^{(2)}\left(\Gamma_{t} \Delta_{t}^{(2, C)}(F)\right)
\end{aligned}
$$

In particular, from (2.4.27) applied with $\widetilde{\Gamma}_{t}=\Gamma_{t} 1_{\left|X_{t}\right| \leq 1}$ and since $1_{g_{C} \leq t} \cdot 1_{\left|X_{t}\right| \leq 1}=0, \Sigma_{t}^{(2, C)}(F)$ vanishes on the set $\left(\left|X_{t}\right| \leq 1\right)$. 
ii) The Doob-Meyer decompositions of $\Sigma_{t}^{(2, C)}(F)$ and $\Delta_{t}^{(2, C)}(F)$ write :

$$
\begin{aligned}
& \Sigma_{t}^{(2, C)}(F)=-M_{t}^{\Sigma^{(2, C)}}(F)+\int_{0} k_{s}^{(C)}(F) d L_{s}^{(C)} \\
& \Delta_{t}^{(2, C)}(F)=M_{t}^{\Delta^{(2, C)}}(F)-\int_{0} k_{s}^{(C)}(F) d L_{s}^{(C)}
\end{aligned}
$$

where $\left(M_{t}^{\Sigma^{(2, C)}}(F), t \geq 0\right)$ and $\left(M_{t}^{\Delta^{(2, C)}}(F), t \geq 0\right)$ are the martingale parts of the corresponding left-hand sides. The first martingale is not uniformly integrable; the second one is uniformly integrable. In fact, we have:

$$
M_{t}^{\Delta^{(2, C)}}(F)=W^{(2)}\left(\int_{0}^{\infty} k_{s}^{(C)}(F) d L_{s}^{(C)} \mid \mathcal{F}_{t}\right)
$$

with, from (2.4.20), $\int_{0}^{\infty} k_{s}^{(C)}(F) d L_{s}^{(C)} \in L^{1}\left(\mathcal{F}_{\infty}, W^{(2)}\right)$.

iii) The "explicit formula" :

$$
\Sigma_{t}^{(2, C)}(F)=\frac{1}{\pi} \log ^{+}\left(\left|X_{t}\right|\right) \cdot \widehat{\widetilde{E}}_{X_{t}\left(\omega_{t}\right)}^{(2, \log )}\left(F\left(\omega_{t}, \widehat{\omega}^{t}\right)\right)
$$

holds, where in (2.4.32) the expectation is taken with respect to $\widehat{\omega}^{t}$, and the argument $\omega_{t}$ is frozen. $\widetilde{E}^{(2, \log )}$ denotes the expectation with respect to the law $\widetilde{P}^{(2, \log )}$ defined in Theorem 2.2.2. In particular:

- $\Sigma_{t}^{(2, C)}$ vanishes on $\left\{t ;\left|X_{t}\right| \leq 1\right\}$, as we already observed,

- $\pi \frac{\Sigma_{t}^{(2, C)}(F)}{1+\log ^{+}\left(\left|X_{t}\right|\right)} \underset{t \rightarrow \infty}{\longrightarrow} F \quad \mathbf{W}^{(2)}$ a.s.

and, from (2.4.16)

$$
\pi \frac{\Delta_{t}^{(2, C)}(F)}{1+\log ^{+}\left(\left|X_{t}\right|\right)} \underset{t \rightarrow \infty}{\longrightarrow} 0 \quad \mathbf{W}^{(2)} \text { a.s. }
$$

Corollary 2.4.5. Let $F \in L^{1}\left(\mathcal{F}_{\infty}, \mathbf{W}^{(2)}\right)$.

The martingale $\left(M_{t}(F), t \geq 0\right)$ is such that : for every $t \geq 0$,

$M_{t}^{(2)}(F)=0$ for every $t$ such that $\left|X_{t}\right| \geq 1$

if and only if :

$$
k_{g_{C}}^{(C)}(F)=0
$$




\section{Chapter 3. The analogue of the measure $\mathrm{W}$ for a class of linear diffusions.}

In Chapters 1 and 2, we have, starting from penalisation results, associated to Wiener measure in dimensions 1 and 2 a positive and $\sigma$-finite measure $\mathbf{W}$ (resp. : $\mathbf{W}^{(2)}$ in dimension 2 ) on the canonical space $\left(\Omega, \mathcal{F}_{\infty}\right)$. In this $3^{\text {rd }}$ Chapter, we shall prove the existence of a measure which is analogous to $\mathbf{W}$, in the more general situation of a large class of linear diffusions. This class is described in Section 3.2. Our approach in this Chapter does not use any penalisation result. Then, in Section 3.3, we shall particularize these results about linear diffusions to the situation of Bessel processes with dimension $d=2(1-\alpha)(0<d<2$, or $0<\alpha<1)$. Thus, we shall obtain the existence of the measure $\mathbf{W}^{(-\alpha)}(0<\alpha<1)$ on $\left(\mathcal{C}\left(\mathbb{R} \rightarrow \mathbb{R}_{+}\right), \mathcal{F}_{\infty}\right)$ and we shall then indicate its relationship with penalisation problems. Section 3.1 is devoted to a presentation of our hypotheses and notations.

\subsection{Main hypotheses and notations.}

3.1.1 Our framework is that of Salminen-Vallois-Yor. [SVY], that is :

$\left(X_{t}, t \geq 0\right)$ is a $\mathbb{R}_{+}=[0, \infty$ [ valued diffusion, with 0 an instantaneously reflecting barrier. The infinitesimal generator $L$ of $\left(X_{t}, t \geq 0\right)$ is given by :

$$
L f(x)=\frac{d}{d m} \frac{d}{d S} f(x) \quad(x \geq 0)
$$

where $S$ is a continuous, strictly increasing function s.t. :

$$
S(0)=0, \quad S(+\infty)=+\infty
$$

and $m(d x)$ is the speed measure of $X$; we assume $m(\{0\})=0$.

3.1.2 The semi-group of $\left(X_{t}, t \geq 0\right)$ admits $p(t, x, y)$ as density with respect to $m$ :

$$
P_{x}\left(X_{t} \in d y\right)=p(t, x, y) m(d y)
$$

with $p$ continuous in the 3 variables, and $p(t, x, y)=p(t, y, x) . \widehat{X}$ denotes the process $X$, killed at $T_{0}=\inf \left\{t ; X_{t}=0\right\}$. We denote by $\widehat{p}$ its density with respect to $m$ :

$$
\widehat{P}_{x}\left(\widehat{X}_{t} \in d y\right)=P_{x}\left(X_{t} \in d y ; 1_{t<T_{0}}\right):=\widehat{p}(t, x, y) m(d y)
$$

3.1.3 We denote by $\left\{L_{t}^{y} ; t \geq 0, y \geq 0\right\}$ the jointly continuous family of local times of $X$, which satisfy the density of occupation formula:

$$
\int_{0}^{t} h\left(X_{s}\right) d s=\int_{0}^{\infty} h(y) L_{t}^{y} m(d y)
$$

for any $h: \mathbb{R}_{+} \rightarrow \mathbb{R}_{+}$, Borel. It is easily deduced from (3.1.5) and (3.1.3) that:

$$
E_{x}\left(d_{t} L_{t}^{y}\right)=p(t, x, y) d t
$$

We denote by $P_{0}^{\tau_{l}}$ the law, under $P_{0}$, of $\left(X_{t}, t \leq \tau_{l}\right)$ with $\tau_{l}:=\inf \left\{t \geq 0 ; L_{t}^{0}>l\right\}$. We have also :

$$
\left(S\left(X_{t}\right)-L_{t}, t \geq 0\right) \quad \text { is a martingale }
$$


a property which results from (3.1.1) and (3.1.5) (see [DM, RVY] for such a property in the context of Bessel processes).

3.1.4 The process $X$, conditioned not to vanish, is a Doob $h$-transform of $\widehat{X}$, with $h(x)=$ $S(x)$. In other terms : if $P_{x}^{\uparrow}$ is the law of $X$ conditioned not to vanish :

$$
P_{x}^{\uparrow}\left(F_{t}\right)=\frac{1}{S(x)} E_{x}\left[F_{t} S\left(X_{t}\right) 1_{t<T_{0}}\right]
$$

for any $F_{t} \in b\left(\mathcal{F}_{t}\right)$. In particular, the semi-group of the conditioned process is given by :

$$
P_{x}^{\uparrow}\left(X_{t} \in d y\right)=\frac{\widehat{p}(t, x, y)}{S(x)} S(y) m(d y) \quad(x \geq 0)
$$

Later, it will be interesting to use the following :

$$
P_{0}^{\uparrow}\left(X_{t} \in d y\right)=f_{y, 0}(t) S(y) m(d y)
$$

where $f_{y, 0}(t)$ admits the following description :

$$
\begin{aligned}
& f_{y, 0}(t)=\lim _{x \downarrow 0} \frac{\widehat{p}(t, x, y)}{S(x)} \\
& f_{y, 0}(t) d t \underset{(a)}{=} P_{y}\left(T_{0} \in d t\right) \underset{(b)}{=} P_{0}^{\uparrow}\left(g_{y} \in d t\right)
\end{aligned}
$$

with

$$
g_{y}:=\sup \left\{t ; X_{t}=y\right\}
$$

We indicate here that (3.1.2) is a partial expression of the time reversal result :

$$
P_{y}\left(\left\{X_{T_{0}-t}, t \leq T_{0}\right\}\right)=P_{0}^{\uparrow}\left(\left\{X_{u}, u \leq g_{y}\right\}\right)
$$

Furthermore :

$$
P_{0}^{\uparrow}\left\{\left\{X_{u}, u \leq g_{y}\right\} \mid g_{y}=t\right\}=P_{0}^{\uparrow}\left(\left\{X_{u}, u \leq t\right\} \mid X_{t}=y\right)
$$

where in (3.1.13) and in (3.1.14) we have used the notation $P\left(\left\{X_{u}, u \leq a\right\}\right)$ to denote the law of the process $\left(X_{u}, u \leq a\right)$ under $P$. All these facts, as well as those presented in the following Proposition may be found in $[\mathrm{SVY}],[\mathrm{S}],[\mathrm{B}-\mathrm{S}],[\mathrm{PY}], \ldots$ which all deal with properties of linear diffusions.

3.1.5 A useful Proposition :

We shall use the following :

Proposition 3.1.1. Let $g^{(t)}:=\sup \left\{s \leq t, X_{s}=0\right\}$.

1) Under $P_{0}$, conditionally on $g^{(t)}$ the processes $\left(X_{u}, u \leq g^{(t)}\right)$ and $\left(X_{g^{(t)}+u}, u \leq t-g^{(t)}\right)$ are independent.

2) Conditionally on $g^{(t)}=s,(s \leq t)$ the process $\left(X_{u}, u \leq s\right)$ is distributed as $\Pi_{0}^{(s)}$, the law of the bridge of $X$ under $P_{0}$, with length $s$, ending at $x=0$ at time $s$.

3) The law of the couple $\left\{g^{(t)},\left(X_{g^{(t)}+u}, u \leq t-g^{(t)}\right)\right\}$ under $P_{0}$ may be described as follows :

i) $\quad P_{0}\left(g^{(t)} \in d s, X_{t} \in d y\right) S(y)=p(s, 0,0) 1_{s<t} P_{0}^{\uparrow}\left(X_{t-s} \in d y\right) d s$ or equivalently, with the help of (3.1.10):

$\left.i^{\prime}\right) \quad P_{0}\left(g^{(t)} \in d s, X_{t} \in d y\right)=p(s, 0,0) f_{y, 0}(t-s) 1_{s<t} d s m(d y)$ 
and, on the other hand:

ii) $\quad P_{0}\left(\left\{X_{g_{t}+u}, u \leq t-g_{t}\right\} \mid X_{t}=y, g_{t}=s\right)$

$$
=P_{0}^{\uparrow}\left(\left\{X_{u}, u \leq t-s\right\} \mid X_{t-s}=y\right)
$$

There different properties are established in [SVY] and [S].

\subsection{The $\sigma$-finite measure $\mathrm{W}^{*}$.}

3.2.1 Definition of $\mathbf{W}^{*}$ :

Here is the main result of this Section.

Theorem 3.2.1.

1) There exists a unique $\sigma$-finite measure, which we denote by $\mathbf{W}^{*}$, on $\left(\mathcal{C}\left(\mathbb{R}_{+}, \mathbb{R}_{+}\right), \mathcal{F}_{\infty}\right)$ such that:

$$
\begin{aligned}
& \forall t \geq 0, \forall F_{t} \in b\left(\mathcal{F}_{t}\right): \\
& E_{0}\left(F_{t} S\left(X_{t}\right)\right)=\mathbf{W}^{*}\left(F_{t} 1_{g \leq t}\right)
\end{aligned}
$$

with $g:=\sup \left\{t \geq 0 ; X_{t}=0\right\}$

$$
\begin{array}{ll}
\text { 2) } & \mathbf{W}^{*}=\int_{0}^{\infty} d l\left(P_{0}^{\tau_{l}} \circ P_{0}^{\uparrow}\right) \\
\text { 3) } & \mathbf{W}^{*}=\int_{0}^{\infty} d t p(t, 0,0)\left(\Pi_{0}^{(t)} \circ P_{0}^{\uparrow}\right)
\end{array}
$$

In particular, if we denote $\mathbf{W}_{g}^{*}$ the restriction of $\mathbf{W}^{*}$ to $\mathcal{F}_{g}$, we have :

$$
\mathbf{W}_{g}^{*}=\int_{0}^{\infty} d l P_{0}^{\tau_{l}}=\int_{0}^{\infty} d t p(t, 0,0) \Pi_{0}^{(t)}
$$

Of course, this Theorem 3.2 has been guessed by comparison with the Brownian situation described in Chapters 1 and 2.

Proof of Theorem 3.2.1.

i) First of all, it is not difficult to show that, starting from equation (3.2.1), where $\mathbf{W}^{*}$ is the unknown, this problem admits at most one solution.

ii) Define

$$
\mathbf{W}_{*}=\int_{0}^{\infty} d l\left(P_{0}^{\tau_{l}} \circ P_{0}^{\uparrow}\right)
$$

We shall now prove that $\mathbf{W}_{*}$ satisfies (3.2.3) and (3.2.4). Since, under $P_{0}^{\uparrow}$, the process $\left(X_{t}, t \geq 0\right)$ remains in $\mathbb{R}_{+} \backslash\{0\}$, it follows immediately, from the definition (3.2.5) of $\mathbf{W}_{*}$ that

$$
\mathbf{W}_{*, g}=\int_{0}^{\infty} d l P_{0}^{\tau_{l}}
$$

On the other hand, a classical argument, which hinges on the fact that the random measure $\left(d L_{s}\right)$ is carried by the zeros of $X$ allows to show easily that :

$$
\int_{0}^{\infty} d l P_{0}^{\tau_{l}}=\int_{0}^{\infty} d t p(t, 0,0) \Pi_{0}^{(t)}
$$

Indeed, by integrating $F:=\left(F_{t}:=F\left(X_{u}, u \leq t\right), t \geq 0\right)$ a positive measurable functional, we obtain on the LHS of (3.2.6)

$$
\int_{0}^{\infty} d l P_{0}^{\tau_{l}}(F)=\int_{0}^{\infty} d l P_{0}\left(F_{\tau_{l}}\right)=P_{0}\left(\int_{0}^{\infty} d L_{s} \cdot F_{s}\right)
$$


by time change $l=L_{s}$

$$
\begin{aligned}
& =P_{0}\left(\int_{0}^{\infty} d L_{s} P_{0}\left(F_{s} \mid X_{s}=0\right)\right) \\
& =\int_{0}^{\infty} P_{0}\left(d L_{s}\right) P_{0}\left(F_{s} \mid X_{s}=0\right) \\
& =\int_{0}^{\infty} d t p(t, 0,0) \Pi_{0}^{(t)}(F)
\end{aligned}
$$

by (3.1.6), with $x=y=0$.

ii) We now prove that $\mathbf{W}_{*}$ satisfies (3.2.1), by showing this equality for the test functions :

$$
F_{t}=\Phi\left(X_{u}, u \leq g^{(t)}\right) \varphi\left(g^{(t)}\right) \psi\left(X_{g^{(t)}+u}, u \leq t-g^{(t)}\right)
$$

From (3.2.3), the RHS of (3.2.1) is equal to (with $\mathbf{W}_{*}$ instead of $\mathbf{W}^{*}$ ) :

$$
\begin{aligned}
R^{F} & :=\mathbf{W}_{*}\left(F_{t} 1_{g \leq t}\right) \\
& =\int_{0}^{t} d s p(s, 0,0) \Pi_{0}^{(s)}\left(\Phi\left(X_{u}, u \leq s\right)\right) \varphi(s) P_{0}^{\uparrow}\left(\psi\left(X_{u}, u \leq t-s\right)\right)
\end{aligned}
$$

On the other hand, the LHS of (3.2.1) is equal to :

$$
\begin{aligned}
L^{F} & :=E_{0}\left[F_{t} S\left(X_{t}\right)\right] \\
& =E_{0}\left[\Phi\left(X_{u}, u \leq g^{(t)}\right) \varphi\left(g^{(t)}\right) \psi\left(X_{g^{(t)}+u}, u \leq t-g^{(t)}\right) S\left(X_{t}\right)\right] \\
& =\int_{0}^{t} P_{0}\left(g^{(t)} \in d s\right) \varphi(s) E_{0}\left[\Phi\left(X_{u}, u \leq s\right) \mid X_{s}=0\right] E_{0}\left[\psi\left(X_{s+u}, u \leq t-s\right)\right. \\
& \left.S\left(X_{t}\right) \mid g^{(t)}=s\right]
\end{aligned}
$$

where we have used a part of the results presented in the Proposition 3.1.1. Comparing (3.2.9) and (3.2.10), we now see that showing equality $R^{F}=G^{F}$ (i.e. the proof of (3.2.1) has now been reduced to showing :

$$
\begin{aligned}
& P_{0}^{\uparrow}\left(\psi\left(X_{u}, u \leq t-s\right) p(s, 0,0) 1_{s<t} d s\right. \\
= & P_{0}\left(g^{(t)} \in d s\right) E_{0}\left(\psi\left(X_{s+u} ; u \leq t-s\right) \cdot S\left(X_{t}\right) \mid g^{(t)}=s\right)
\end{aligned}
$$

But (3.2.11) is an easy consequence of point 3 of Proposition 3.1.1.

3.2.2 Some properties of $\mathbf{W}^{*}$.

The end of this subsection 3.2.2 is devoted to the statement of some results related to the measure $\mathbf{W}^{*}$. These results are presented without proofs since those are close to the ones found in Chapter 1. These theorems (below) are due to Christophe Profeta (thesis in preparation).

3.2.2.1 The probabilites $P_{x, \infty}^{(\lambda)}$.

Theorem 3.2.2.

1) Let, for $\lambda \geq 0$ and $x \geq 0$ :

$$
M_{t}^{(\lambda, x)}:=\frac{1+\frac{\lambda}{2} S\left(X_{t}\right)}{1+\frac{\lambda}{2} S(x)} e^{-\frac{\lambda}{2} L_{t}}=1+\frac{\lambda}{2+\frac{\lambda}{2} S(x)} \int_{0}^{t} e^{-\frac{\lambda}{2} L_{s}} d N_{s}
$$


where $\left(N_{s}:=S\left(X_{s}\right)-L_{s}, s \geq 0\right)$ is the martingale defined by (3.1.7). Then, $\left(M_{t}^{(\lambda, x)}, t \geq 0\right)$ is a $\left(\left(\mathcal{F}_{t}, t \geq 0\right), P_{x}\right)$ positive martingale such that : $M_{t}^{(\lambda, x)} \underset{t \rightarrow \infty}{\longrightarrow} 0$, a.s.

2) Let us define the probability $P_{x, \infty}^{(\lambda)}$ by :

$$
\left.P_{x, \infty}^{(\lambda)}\right|_{\mathcal{F}_{t}}=\left.M_{t}^{(\lambda, x)} \cdot P_{x}\right|_{\mathcal{F}_{t}}
$$

Then, under $P_{x, \infty}^{(\lambda)}$ :

- The canonical process $\left(X_{t}, t \geq 0\right)$ is a transient diffusion with infinitesimal generator $L_{\infty}^{(\lambda)}$ :

$$
\begin{aligned}
L_{\infty}^{(\lambda)} f(x) & =\frac{2}{2+\lambda S(x)}\left(L f(x)+\frac{\lambda}{2} L(S f)(x)\right) \\
& =L f(x)+\frac{2 \lambda}{2+\lambda S(x)} \frac{d f}{d m}(x)
\end{aligned}
$$

and scale function $S_{\infty}^{(\lambda)}$ :

$$
S_{\infty}^{(\lambda)}:=-\frac{2}{2+\lambda S}
$$

- If $\alpha<\lambda$ :

$$
E_{x, \infty}^{(\lambda)}\left(e^{\frac{\alpha}{2} L_{\infty}}\right)<\infty
$$

and if $\alpha \geq \lambda$ :

$$
E_{x, \infty}^{(\lambda)}\left(e^{\frac{\alpha}{2} L_{\infty}}\right)=\infty
$$

- The law of $L_{\infty}$ is given by :

$$
P_{x, \infty}^{(\lambda)}\left(L_{\infty} \in d l\right)=\frac{\lambda}{2+\lambda S(x)} e^{-\frac{\lambda}{2} l} d l+S(x) \delta_{0}(d l)
$$

- $P_{x, \infty}^{(\lambda)}$ admits the following decomposition :

$$
\begin{aligned}
P_{x, \infty}^{(\lambda)} & =\frac{\lambda}{2+\lambda S(x)} \int_{0}^{\infty} d u p(u, x, 0) e^{-\frac{\lambda}{2} L_{u}} \Pi_{x, 0}^{(u)} \circ P_{0}^{\uparrow}+\frac{\lambda S(x)}{2+\lambda S(x)} P_{x}^{\uparrow} \\
& =\frac{\lambda}{2+\lambda S(x)} \int_{0}^{\infty} e^{-\frac{\lambda l}{2}} d l P_{x}^{\tau_{l}} \circ P_{0}^{\uparrow}+\frac{\lambda S(x)}{2+\lambda S(x)} P_{x}^{\uparrow}
\end{aligned}
$$

3.2.2.2 The measures $\left(\mathbf{W}_{x}^{*}, x \in \mathbb{R}_{+}\right)$.

\section{Theorem 3.2.3.}

1) For any $\lambda>0$, the $\sigma$-finite measure $\left(\frac{2}{\lambda}+S(x)\right) \cdot e^{\frac{\lambda}{2} L_{\infty}} \cdot P_{x, \infty}^{(\lambda)}$ does not depend on $\lambda$. We define :

$$
\mathbf{W}_{x}^{*}:=\left(\frac{2}{\lambda}+S(x)\right) e^{\frac{\lambda}{2} L_{\infty}} \cdot P_{x, \infty}^{(\lambda)}
$$


We have the decompositions:

$$
\begin{aligned}
\mathbf{W}_{x}^{*} & =\int_{0}^{\infty} d u p(u, x, 0) \Pi_{x, 0}^{(u)} \circ P_{0}^{\uparrow}+S(x) P_{x}^{\uparrow} \\
\text { and } \quad \mathbf{W}_{x}^{*} & =\int_{0}^{\infty} d l P_{x}^{\tau_{l}} \circ P_{0}^{\uparrow}+S(x) P_{x}^{\uparrow}
\end{aligned}
$$

In particular, $\mathbf{W}_{0}^{*}=\mathbf{W}^{*}$, where $\mathbf{W}^{*}$ is defined by (3.22) or (3.23).

2) - For every $\left(\mathcal{F}_{t}, t \geq 0\right)$ stopping time $T$ and $\Gamma_{T} \in b\left(\mathcal{F}_{T}\right)$ :

$$
E_{x}\left(\Gamma_{T} S\left(X_{T}\right) 1_{T<\infty}\right)=\mathbf{W}_{x}^{*}\left(\Gamma_{T} 1_{g \leq T<\infty}\right)
$$

where $g:=\sup \left\{s \geq 0 ; X_{s}=0\right\}$

- The law of $g$ is given by:

$$
\mathbf{W}_{x}^{*}(g \in d t)=p(t, x, 0) d t+S(x) \delta_{0}(d t) \quad(t \geq 0)
$$

and for every $\left(\mathcal{F}_{t}, t \geq 0\right)$ stopping time $T$, we have :

$$
\mathbf{W}_{x}^{*}\left(1_{T<\infty}, L_{\infty}-L_{T} \in d l\right)=P_{x}(T<\infty) d l+E_{x}\left[S\left(X_{t}\right) \delta_{0}(d l)\right]
$$

3) For every predictable and positive process $\left(\Phi_{s}, s \geq 0\right)$, we have :

$$
\mathbf{W}_{x}^{*}\left(\Phi_{g}\right)=S(x) \Phi_{0}+E_{x}\left(\int_{0}^{\infty} \Phi_{s} d L_{s}\right)
$$

3.2.2.3 Martingales associated with $\left(\mathbf{W}_{x}^{*}, x \in \mathbb{R}_{+}\right)$.

\section{Theorem 3.2.4.}

Let $F \in L_{+}^{1}\left(\Omega, \mathcal{F}_{\infty}, \mathbf{W}_{x}^{*}\right)$. There exists a positive $\left(\left(\mathcal{F}_{t}, t \geq 0\right), P_{x}\right)$ martingale $\left(M_{t}^{*}(F), t \geq 0\right)$ such that:

1) For every $t \geq 0$ and $\Gamma_{t} \in b\left(\mathcal{F}_{t}\right)$ :

$$
\mathbf{W}_{x}^{*}\left(F \cdot \Gamma_{t}\right)=E_{x}\left(M_{t}^{*}(F) \Gamma_{t}\right)
$$

In particular, $\mathbf{W}_{x}^{*}(F)=E_{x}\left(M_{t}^{*}(F)\right)$

2) For every $\lambda>0$ :

$$
\begin{aligned}
M_{t}^{*}(F) & =\left(\frac{2}{\lambda}+S\left(X_{t}\right)\right) e^{-\frac{\lambda}{2} L_{t}} E_{x, \infty}^{(\lambda)}\left(F e^{\left.\frac{1}{2} L_{\infty} \mid \mathcal{F}_{t}\right)}\right. \\
& =\widehat{\mathbf{W}}_{X_{t}}^{*}\left(F\left(\omega_{t}, \widehat{\omega}^{t}\right)\right)
\end{aligned}
$$

3) $M_{t}^{*}(F) \underset{t \rightarrow \infty}{\longrightarrow} 0 \quad P_{x} \quad$ a.s.

\section{Examples :}

- Let $h: \mathbb{R}_{+} \rightarrow \mathbb{R}_{+}$such that $\int_{0}^{\infty} h(u) d u<\infty$. Then :

$$
M_{t}^{*}\left(h\left(L_{\infty}\right)\right)=h\left(L_{t}\right) S\left(X_{t}\right)+\int_{L_{t}}^{\infty} h(l) d l
$$


In particular, if $h(y)=e^{-\frac{\lambda}{2} y}(y \geq 0)$ :

$$
M_{t}^{*}\left(e^{-\frac{\lambda}{2} L_{\infty}}\right)=\left(\frac{2}{\lambda}+S\left(X_{t}\right)\right) e^{-\frac{\lambda}{2} L_{t}}=\frac{2}{\lambda} M_{t}^{(\lambda, 0)} \quad(x=0)
$$

- $\Phi: \mathbb{R}_{+} \rightarrow \mathbb{R}_{+}$Borel such that $\int_{0}^{\infty} \Phi(u) p(u, x, 0) d u<\infty$. Then

$$
M_{t}^{*}(\Phi(g))=\Phi\left(g^{(t)}\right) S\left(X_{t}\right)+\int_{0}^{\infty} \Phi(t+u) p\left(u, X_{t}, 0\right) d t
$$

3.2.2.4 A Theorem of decomposition of $\left(\left(\mathcal{F}_{t}, t \geq 0\right), P_{x}\right)$ supermartingales.

\section{Theorem 3.2.5.}

Let $\left(Z_{t}, t \geq 0\right)$ a positive $\left(\left(\mathcal{F}_{t}, t \geq 0\right), P_{x}\right)$ supermartingale. We denote

$$
Z_{\infty}:=\lim _{t \rightarrow \infty} Z_{t} \quad P_{x} \quad \text { a.s. }
$$

Then:

1) $z_{\infty}:=\lim _{t \rightarrow \infty} \frac{Z_{t}}{1+S\left(X_{t}\right)}$ exists $\mathbf{W}_{x}^{*}$ a.s. and $\mathbf{W}_{x}^{*}\left(z_{\infty}\right)<\infty$

2) $\left(Z_{t}, t \geq 0\right)$ admits the following decomposition:

$$
Z_{t}=M_{t}^{*}\left(z_{\infty}\right)+E_{x}\left(Z_{\infty} \mid \mathcal{F}_{t}\right)+\xi_{t}
$$

where $\left(M_{t}^{*}\left(z_{\infty}\right), t \geq 0\right)$ and $E_{x}\left(Z_{\infty} \mid \mathcal{F}_{t}\right)$ denote two positive $\left(\left(\mathcal{F}_{t}, t \geq 0\right), P_{x}\right)$ martingales and $\left(\xi_{t}, t \geq 0\right)$ is a positive supermartingale such that:

- $Z_{\infty} \in L^{1}\left(\mathcal{F}_{\infty}, P_{x}\right)$, hence $\left(E_{x}\left(Z_{\infty} \mid \mathcal{F}_{t}\right), t \geq 0\right)$ is a uniformly integrable martingale conver ging toward $Z_{\infty}$.

- $\frac{E_{x}\left(Z_{\infty} \mid \mathcal{F}_{t}\right)+\xi_{t}}{1+S\left(X_{t}\right)} \underset{t \rightarrow \infty}{\longrightarrow} 0 \quad \mathbf{W}_{x}^{*}$ a.s.

- $M_{t}^{*}\left(z_{\infty}\right)+\xi_{t} \underset{t \rightarrow \infty}{\longrightarrow} 0 \quad P_{x}$ a.s.

This decomposition is unique

\section{Corollary 3.2.6.}

A positive martingale $\left(Z_{t}, t \geq 0\right)$ is equal to $\left(M_{t}^{*}(F), t \geq 0\right)$ for some $F \in L_{+}^{1}\left(\mathcal{F}_{\infty}, \mathbf{W}_{x}^{*}\right)$ if and only if :

$$
Z_{0}=\mathbf{W}_{x}^{*}\left(\lim _{t \rightarrow \infty} \frac{Z_{t}}{1+S\left(X_{t}\right)}\right)
$$

In the present framework of linear diffusions, it is possible to state a decomposition theorem for the martingales $\left(M_{t}^{*}(F), t \geq 0\right)\left(F \in L^{1}\left(\mathcal{F}_{\infty}, \mathbf{W}_{x}^{*}\right)\right)$ which is similar to Theorem 1.2.11. We leave this task to the interested reader.

3.2.3 Relation between the measure $\mathbf{W}^{*}$ and penalistions.

In a work in preparation (see $[\mathrm{S}, \mathrm{V}]$ ), P. Salminen and P. Vallois obtain the following result, which we now summarize :

Let $\left(\tau_{l}, l \geq 0\right)$ the right continuous inverse of the local time process $\left(L_{t}, t \geq 0\right)$ at level 0 associated to $\left(X_{t}, t \geq 0\right)$ :

$$
\tau_{l}:=\inf \left\{t \geq 0 ; L_{t}>l\right\}
$$


This subordinator $\left(\tau_{l}, l \geq 0\right)$ admits as a Levy measure a mesure $\nu$ with density, which we denote here by $\dot{\nu}($ see $[\mathrm{K} . \mathrm{S}])$ :

$$
E\left(e^{-\lambda \tau_{l}}\right)=\exp \left\{-l \int_{0}^{\infty}\left(1-e^{-\lambda x}\right) \dot{\nu}(x) d x\right\} \quad(\lambda, l \geq 0)
$$

P. Salminen and P. Vallois then make the following hypothesis : the function $F:[1, \infty[\rightarrow[0,1]$ defined by :

$$
F(x):=\frac{\nu(] 1, x[)}{\nu(] 1, \infty[)}=\frac{\int_{1}^{x} \dot{\nu}(y) d y}{\int_{1}^{\infty} \dot{\nu}(y) d y}
$$

is sub-exponential, i.e. :

$$
\lim _{x \rightarrow \infty} \frac{\overline{F * F}(x)}{\bar{F}(x)}=2
$$

where $\bar{F}(x):=1-F(x) x \geq 1$ and where $*$ indicates the convolution operation.

One of the main consequences of the subexponentiality of $F$ is :

$$
\frac{\bar{F}(x+y)}{\bar{F}(x)} \underset{x \rightarrow \infty}{\longrightarrow} 1 \quad \text { uniformly on compacts (in } y \text { ) }
$$

Thus, here

$$
\frac{\nu(] x+y, \infty[)}{\nu(] x, \infty[)} \underset{x \rightarrow \infty}{\longrightarrow} 1 \quad \text { uniformly on compacts (in } y \text { ) }
$$

Under this hypothesis of subexponentiality, P. Salminen and P. Vallois then prove the following Theorem.

Theorem 3.2.7. (Penalisation by $\left(1_{\left(L_{t}<l\right)}, t \geq 0\right)$

Let $l>0$ be fixed. Then, for every $s \geq 0$ and $\Gamma_{s} \in b\left(\mathcal{F}_{s}\right)$ :

$$
\lim _{t \rightarrow \infty} \frac{E_{x}\left(\Gamma_{s} 1_{\left(L_{t}<l\right)}\right)}{P_{x}\left(L_{t}<l\right)}=E_{x}\left(\Gamma_{s} \cdot M_{s}^{(l)}\right):=P_{x, \infty}^{(l)}\left(\Gamma_{s}\right)
$$

where $\left(M_{s}^{(l)}, s \geq 0\right)$ is the positive martingale defined by :

$$
M_{s}^{(l)}:=\frac{S\left(X_{s}\right)-L_{s}+l}{S(x)+l} \cdot 1_{L_{s}<l}
$$

The key point of the proof of Theorem 3.2.7 is the following

Lemma 3.2.8. ([S.V])

$$
P_{x}\left(L_{t}<l\right) \underset{t \rightarrow \infty}{\sim}(S(x)+l) \nu(] t, \infty[)
$$

Theorem 3.2.7 now follows easily from Lemma 3.2.8 and from relation (3.2.37).

From this Theorem 3.2.7, we deduce the following relation between the probability $P_{0, \infty}^{(l)}$ defined by (3.2.38) and the $\sigma$-finite measure $\mathbf{W}^{*}$ defined by (3.2.2) or (3.2.3) :

$$
1_{L_{\infty}<l} \cdot \mathbf{W}^{*}=\mathbf{W}^{*}\left(L_{\infty}<l\right) \cdot P_{0, \infty}^{(l)}
$$


(We note that $\left.P_{0, \infty}^{(l)}\left(L_{\infty}<l\right)=1\right)$. The reader may compare relation $(3.2 .39)$ with the relation (1.1.107) of Theorem 1.1.11 and with the relation (3.2.21) of Theorem 3.2.3. From (3.2.39), we also deduce, with the notation of Theorem 3.2.4, that :

$$
M_{t}^{*}\left(1_{\left(L_{\infty}<l\right)}\right)=\mathbf{W}^{*}\left(L_{\infty}<l\right) \cdot\left(\frac{S\left(X_{t}\right)+l-L_{t}}{l}\right) 1_{L_{t}<l} \quad(x=0)
$$

Finally, we indicate that in the same work in progress, C. Profeta, P. Salminen and P. Vallois study the penalisation of reflected Brownian motion on the space interval $[0,1]$ with the functional $\left(1_{\left(L_{\infty}<l\right)}, t \geq 0\right)$. This time, since the measure $m$ is Lebesque measure on $[0,1]$, which does note charge the entire half-line $\mathbb{R}_{+}$, the subexponentiality hypothesis is not satisfied.

3.3 The example of Bessel process in dimension $d, 0<d<2$.

3.3.1 Transcription of our notation in the context of Bessel processes.

Let $d=2(1-\alpha)$ with $0<d<2$ (or $0<\alpha<1)$. We now study the particular case of the process $\left(X_{t}, t \geq 0\right)$ described in Section 3.1 with :

$$
\begin{aligned}
m(d x) & =\frac{x^{1-2 \alpha}}{\alpha} 1_{[0, \infty[}(x) d x \\
S(x) & =x^{2 \alpha} \quad(x \geq 0)
\end{aligned}
$$

Then, the process $\left(X_{t}, t \geq 0\right)$ described in Section 3.1 is a Bessel process with dimension $d$, and index $\frac{d}{2}-1=-\alpha$. We denote by $\left(P_{x}^{(-\alpha)}, x \in \mathbb{R}_{+}\right)$the family of its laws. We note $\left(\Omega=\mathcal{C}\left(\mathbb{R}_{+} \rightarrow \mathbb{R}_{+}\right),\left(R_{t}, \mathcal{F}_{t}\right), t \geq 0, \mathcal{F}_{\infty}, P_{x}^{(-\alpha)}\left(x \in \mathbb{R}_{+}\right)\right)$the canonical realisation of the Bessel process with index $(-\alpha)$. Here, the probability $P_{x}^{\uparrow}$ defined in 3.1.4 is the law of Bessel process with dimension $4-d=2(1+\alpha)$, i.e. : index $\alpha$. We shall denote by $P_{x}^{(\alpha)}$ this law. The formulae of subsection 3.1 now become :

$$
\begin{aligned}
& \left(R_{t}^{2 \alpha}-L_{t}, t \geq 0\right) \text { is a martingale } \\
& P_{x}^{\uparrow}=P_{x}^{(\alpha)} \\
& \int_{0}^{t} h\left(R_{s}\right) d s=\frac{1}{\alpha} \int_{0}^{\infty} h(x) L_{t}^{x} x^{1-2 \alpha} d x \\
& E_{0}^{(-\alpha)}\left(L_{t}^{0}\right)=t^{\alpha} E_{0}^{(-\alpha)}\left(L_{1}\right)=\frac{2^{\alpha} t^{\alpha}}{\Gamma(1-\alpha)} \\
& L^{(\alpha)} f(r)=\frac{1}{2} f^{\prime \prime}(r)+\frac{1+2 \alpha}{2 r} f^{\prime}(r)
\end{aligned}
$$

The reader may refer to [D.M, RVY] for these formulae.

\subsubsection{The measure $\mathbf{W}^{(-\alpha)}$.}

In this framework, Theorem 3.2.1 becomes :

Theorem 3.3.1. For every $\alpha \in] 0,1[$ :

1) There exists a unique positive and $\sigma$-finite measure $\mathbf{W}^{(-\alpha)}$ on $\left(\Omega=\mathcal{C}\left(\mathbb{R}_{+}, \mathbb{R}_{+}\right), \mathcal{F}_{\infty}\right)$ such that,

$$
\mathbf{W}^{(-\alpha)}\left(F_{t} 1_{g \leq t}\right)=P_{0}^{(-\alpha)}\left(F_{t} \cdot R_{t}^{2 \alpha}\right)
$$

2) $\quad \mathbf{W}^{(-\alpha)}=\int_{0}^{\infty}\left(P_{0}^{\left(-\alpha, \tau_{l}\right)} \circ P_{0}^{(\alpha)}\right) d l$ 
3) i) $\quad \mathbf{W}^{(-\alpha)}(g \in d t)=\frac{\alpha 2^{\alpha}}{\Gamma(1-\alpha)} t^{\alpha-1} d t \quad(t \geq 0)$

ii) Conditionally on $g=t$, under $\mathbf{W}^{(-\alpha)},\left(R_{u}, u \leq g\right)$ is a Bessel bridge with index $(-\alpha)$ and of length $t$

iii) $\quad \mathbf{W}^{(-\alpha)}=\int_{0}^{\infty} \frac{\alpha 2^{\alpha} t^{\alpha-1}}{\Gamma(1-\alpha)} d t\left(\Pi_{0}^{(-\alpha, t)} \circ P_{0}^{(+\alpha)}\right)$

In this Theorem :

$\Pi_{0}^{(-\alpha, t)}$ denotes the law of a Bessel bridge with index $(-\alpha)$ and of length $t$.

$P_{0}^{\left(-\alpha, \tau_{l}\right)}$ denotes the law of a Bessel process with index $(-\alpha)$ starting at 0 and stopped at $\tau_{l}$, with :

$$
\tau_{l}=\inf \left\{t \geq 0 ; L_{t}^{0}>l\right\}
$$

3.3.3 Relations between $\mathbf{W}^{(-\alpha)}(d=2(1-\alpha))$ and Feynman-Kac penalisations.

Remark 3.3.2. The measure $\mathbf{W}^{(-\alpha)}$ which we just described is also related to a penalisation problem. More precisely, one can prove (see [RVY, I or V]) :

i) Let $q$ be a positive Radon measure on $\mathbb{R}_{+}$, with compact support. Then :

$$
2^{\alpha} \Gamma(1+\alpha) t^{\alpha} P_{r}^{(-\alpha)}\left(\exp \left(-\frac{1}{2} A_{t}^{(q)}\right)\right) \underset{t \rightarrow \infty}{\longrightarrow} \varphi_{q}^{(-\alpha)}(r)
$$

with

$$
A_{t}^{(q)}:=\int_{0}^{\infty} q\left(R_{s}\right) d s=\frac{1}{\alpha} \int_{0}^{\infty} L_{t}^{x} x^{1-2 \alpha} q(d x)
$$

ii) The function $\varphi_{q}^{(-\alpha)}$ defined by (3.3.12) is characterised as the unique solution of :

$$
\left\{\begin{array}{l}
\frac{1}{2} f^{\prime \prime}(r)+\frac{1-2 \alpha}{2 r} f^{\prime}(r)=\frac{1}{2} f(r) q(r) \\
\text { (in the sense of Schwartz distributions) } \\
f(r) \underset{r \rightarrow \infty}{\sim} r^{2 \alpha}
\end{array}\right.
$$

iii) For every $s \geq 0$ and $\Gamma_{s} \in b\left(\mathcal{F}_{s}\right)$ :

$$
E_{r}^{(-\alpha)}\left(\Gamma_{s} \frac{\exp -\frac{1}{2} A_{t}^{(q)}}{E_{r}^{(-\alpha)}\left(\exp -\frac{1}{2} A_{t}^{(q)}\right)}\right) \underset{t \rightarrow \infty}{\longrightarrow} P_{r, \infty}^{(-\alpha)}\left(\Gamma_{s}\right)
$$

where the probability $P_{r, \infty}^{(-\alpha)}$ satisfies :

$$
\left.P_{r, \infty}^{(-\alpha)}\right|_{\mathcal{F}_{s}}=\left.M_{s}^{(-\alpha, q)} P_{r}^{(-\alpha)}\right|_{\mathcal{F}_{s}}
$$

with

$$
M_{s}^{(-\alpha, q)}=\frac{\varphi_{q}^{(-\alpha)}\left(R_{s}\right)}{\varphi_{q}^{(-\alpha)}(r)} \exp \left(-\frac{1}{2} A_{s}^{(q)}\right)
$$

and $\left(M_{s}^{(-\alpha, q)}, s \geq 0\right)$ is a $\left(\left(\mathcal{F}_{s}, s \geq 0\right), P^{(-\alpha)}\right)$ martingale.

iv) Under $P_{r, \infty}^{(-\alpha, q)}(r \geq 0)$, the canonical process $\left(R_{t}, t \geq 0\right)$ is a transient diffusion with infinitesimal generator $L^{(-\alpha, q)}$ given by :

$$
L^{(-\alpha, q)} f(r)=\frac{1}{2} f^{\prime \prime}(r)+\left(\frac{1-2 \alpha}{2 r}+\frac{\left(\varphi_{q}^{(-\alpha)}\right)^{\prime}}{\varphi_{q}^{(-\alpha)}}(r)\right) f^{\prime}(r)
$$




\section{Remark 3.3.3.}

With the notation of Remark 3.3.2, in the particular case where $q$ is the measure $q_{0}$ such that $\frac{1}{\alpha} x^{1-2 \alpha} q_{0}(d x)$ is Dirac mass in 0 (of course, this is a little formal : we have to chose a sequence $q_{0}^{(n)}$ such that $\frac{1}{\alpha} x^{1-2 \alpha} q_{0}^{(n)}(d x)$ converges towards $\delta_{0}$ as $\left.n \rightarrow \infty\right)$

$$
\begin{aligned}
\varphi_{q_{0}}^{(-\alpha)}(r) & =2+r^{2 \alpha}, \varphi_{q_{0}}^{(-\alpha)}(0)=2 \\
\text { and } \quad M_{t}^{\left(-\alpha, q_{0}\right)} & =\left(1+\frac{R_{t}^{2 \alpha}}{2}\right) e^{-\frac{1}{2} L_{t}}
\end{aligned}
$$

Now, the analogue of Theorem 1.1.5 is :

\section{Theorem 3.3.4.}

Under $P_{\infty}^{\left(-\alpha, q_{0}\right)}$, the canonical process $\left(R_{t}, t \geq 0\right)$ satisfies :

i) Let $g=\sup \left\{s \geq 0, R_{s}=0\right\}$. Then :

$$
g<\infty \quad P_{\infty}^{\left(-\alpha, q_{0}\right)} \quad \text { a.s. } \quad \text { and }
$$

ii) $L_{\infty}\left(=L_{g}\right)$ admits as density :

$$
f_{L_{\infty}}^{P_{\infty}^{\left(-\alpha, q_{0}\right)}}(l)=\frac{1}{2} e^{-\frac{l}{2}} 1_{[0, \infty[}(l) d l
$$

iii) $\left(R_{s}, s \leq g\right)$ and $\left(R_{g+s}, s \geq 0\right)$ are independent.

iv) $\left(R_{g+s}, s \geq 0\right)$ is $a(4-d)$ dimensional Bessel process starting at 0 (i.e. admits $P_{0}^{(+\alpha)}$ as law).

v) Conditionally on $L_{\infty}\left(=L_{g}\right)=l,\left(R_{s}, s \leq g\right)$ is a d-dimensional Bessel process stopped in $\tau_{l}$. Its law is $P_{0}^{\left(-\alpha, \tau_{l}\right)}$.

\section{Remark 3.3.5.}

1) Since, for $\alpha=\frac{1}{2},\left(R_{t}, t \geq 0\right)$ under $P^{(-\alpha)}$ is a reflected Brownian motion, one has :

$$
\mathbf{W}\left(F\left(\left|X_{s}\right|, s \geq 0\right)\right)=\mathbf{W}^{\left(-\frac{1}{2}\right)}\left(F\left(R_{s}, s \geq 0\right)\right)
$$

2) In the same spirit, since the modulus of a 2-dimensional Brownian motion is a 2-dimensional Bessel process, hence has index 0, we conjecture that, in a sense to be made precise :

$$
\mathbf{W}^{(2)}\left(F\left(\left|X_{s}\right|, s \geq 0\right)\right)=\lim _{\alpha \downarrow 0} \mathbf{W}^{(-\alpha)}\left(F\left(R_{s}, s \geq 0\right)\right)
$$

\section{Remark 3.3.6.}

We have given, in sub-section 1.1.6, a proof of Theorem 1.1.6 (this is precisely Theorem 1.1.10) which hinges upon the disintegration of Wiener measure restricted to $\mathcal{F}_{t}$, with respect to the law $g^{(t)}$ (see (1.1.82)). Formula (3.3.11) may be proven in a quite similar way by using the following :

i) For fixed time $t$, the three following random elements are independent :

- $\left(r_{u}:=\frac{1}{\sqrt{g^{(t)}}} R_{u g^{(t)}}, u \leq 1\right) ;$ it is a Bessel bridge with dimension $d=2(1-\alpha)$ 
- $g^{(t)}:=\sup \left\{u<t ; R_{u}=0\right\}$, it is distributed as :

$$
P_{0}^{(-\alpha)}\left(g^{(t)} \in d u\right)=\frac{d u}{b_{\alpha} u^{1-\alpha}(t-u)^{\alpha}} \quad(0 \leq u \leq t)
$$

with : $b_{\alpha}=B(\alpha, 1-\alpha)=\Gamma(\alpha) \Gamma(1-\alpha)$

- $\left(m_{u}:=\frac{1}{\sqrt{t-g^{(t)}}} R_{g^{(t)}+u\left(1-g^{(t)}\right)}, u \leq 1\right) ;$ it is a Bessel meander (with dimension $d$ ).

ii) Imhof's absolute continuity relationship between the laws of the Bessel meander $\left(m_{u}, u \leq 1\right)$ and the Bessel process with dimension $2(1+\alpha)$ (i.e. : with index $\alpha$ ).

$$
E^{(-\alpha)}\left(F\left(m_{u}, u \leq 1\right)\right)=E_{0}^{(\alpha)}\left(F\left(R_{u}, u \leq 1\right) \frac{2^{\alpha} \Gamma(1+\alpha)}{R_{1}^{2 \alpha}}\right)
$$

\subsection{Another description of $\mathbf{W}^{(-\alpha)}$ (and $\mathbf{W}_{g}^{*}$ ).}

3.4.1 We recall that (see (3.2.4) and (3.3.9) and (3.3.10)) :

$$
\mathbf{W}_{g}^{*}=\int_{0}^{\infty} d l P_{0}^{\tau_{l}}=\int_{0}^{\infty} d t p(t, 0,0) \Pi_{0}^{(t)}
$$

in the context of general linear diffusions and :

$$
\mathbf{W}_{g}^{(-\alpha)}=\int_{0}^{\infty} d l P_{0}^{\left(-\alpha, \tau_{l}\right)}=\int_{0}^{\infty} \frac{\alpha 2^{\alpha} t^{\alpha-1}}{\Gamma(1-\alpha)} \Pi_{0}^{(-\alpha, t)}
$$

in the context of Bessel processes with index $(-\alpha)(0<\alpha<1)$

We shall now give a new description of $\mathbf{W}_{g}^{(-\alpha)}$ (resp. $\mathbf{W}_{g}^{*}$ ) which is the restriction of $\mathbf{W}_{g}^{(-\alpha)}$ (resp. $\mathbf{W}^{*}$ ) to $\mathcal{F}_{g}$. This new description is just the transcript, in the Bessel framework of results found in Pitman-Yor (see $[\mathrm{PY}]$ ).

3.4.2 We begin by recalling in the framework of Bessel processes some of the results from [PY]. We denote by $\widehat{\Omega}$ the space of continuous functions from $\mathbb{R}_{+}$to $\mathbb{R}_{+}$with finite lifetime $\xi$ :

$$
\begin{gathered}
\widehat{\Omega}=\left\{\omega ; \mathbb{R}_{+} \rightarrow \mathbb{R}_{+} ; \exists \xi(\omega)<\infty \text { t.q } \omega(0)=0=\omega(\xi),\right. \\
\text { and } \quad \omega(u)=0 \text { for every } u \geq \xi(\omega)\}
\end{gathered}
$$

We denote by $\left(R_{t}, t \geq 0\right)$ the set of coordinates on this space :

$$
R_{t}(\omega)=\omega(t), \omega \in \widehat{\Omega}
$$

The result of Pitman-Yor which we use (Theorem 1.1 of [P.Y 2]) asserts the existence, for every $\delta>0$, of a positive and $\sigma$-finite measure on $\left(\widehat{\Omega}, \mathcal{F}_{\infty}\right)$, denoted as $\lambda_{00}^{(\delta)}$ and which may be described in either of the following manners :

First description

$$
\boldsymbol{\Lambda}_{00}^{(\delta)}=\int_{0}^{\infty} \frac{2^{-\frac{\delta}{2}}}{\Gamma(\delta / 2)} t^{-\frac{\delta}{2}} \Pi_{0}^{\left(\frac{\delta}{2}-1, t\right)} d t
$$


where $\Pi_{0}^{\left(\frac{\delta}{2}-1, t\right)}$ denotes the law of the Bessel bridge with index $\frac{\delta}{2}-1$, i.e. with dimension $\delta$ and length 1.

Second description

Let $m>0$ fixed and $P_{0}^{\left(\frac{\delta}{2}-1, m, \swarrow \searrow\right)}$ denote the law of the process obtained by putting two Bessel processes with index $\left(\frac{\delta}{2}-1\right)$ i.e. : with dimension $\delta$, back to back starting from 0 , and stopped when they reach level $m$. These two processes $R$ and $\widetilde{R}$ are assumed to be independent. In other terms, $P_{0}^{\left(\frac{\delta}{2}-1, m, \swarrow\right)}$ is the law of the process $\left(Y_{t}, t \geq 0\right)$ defined by :

$$
Y_{t}= \begin{cases}R_{t} & \text { if } t \leq T_{m} \\ m-\widetilde{R}_{t-T_{m}} & \text { if } \quad T_{m} \leq t \leq T_{m}+\widetilde{T}_{m} \\ 0 & \text { if } t \geq T_{m}+\widetilde{T}_{m}\end{cases}
$$

where $T_{m}\left(\operatorname{resp} \widetilde{T}_{m}\right)$ is the first hitting time of $m$ by $\left(R_{t}, t \geq 0\right)\left(\operatorname{resp}\right.$. by $\left.\left(\widetilde{R}_{t}, t \geq 0\right)\right)$. Then :

$$
\boldsymbol{\Lambda}_{0,0}^{(\delta)}=\int_{0}^{\infty} m^{1-\delta} d m P^{\left(\frac{\delta}{2}-1, m, \swarrow\right)}
$$

The measure $\boldsymbol{\Lambda}_{0,0}^{(\delta)}$ is called the "generalized excursion measure" in Pitman-Yor. When $\delta=3$, $\lambda_{0,0}^{(3)}$ is the Itô measure of (positive) Brownian excursions. Formula (3.4.3) is Itô's description of Itô's measure, whereas formula (3.4.5) is William's description of that measure.

3.4.3 Here is now, in the framework of the Bessel processes, the announced transcription : Theorem 3.4.1 For every $\alpha \in] 0,1[$ :

$$
\left.\mathbf{W}^{-(\alpha)}\right|_{\mathcal{F}_{g}}=\mathbf{W}_{g}^{(-\alpha)}=2 \alpha \lambda_{0,0}^{(2(1-\alpha))}
$$

In particular :

$$
\begin{aligned}
\left.\mathbf{W}^{(-\alpha)}\right|_{\mathcal{F}_{g}} & =\frac{\alpha 2^{\alpha}}{\Gamma(1-\alpha)} \int_{0}^{\infty} t^{\alpha-1} d t \Pi_{0}^{(-\alpha, t)} \\
\left.\mathbf{W}^{(-\alpha)}\right|_{\mathcal{F}_{g}} & =2 \alpha \int_{0} m^{2 \alpha-1} d m P^{(-\alpha, m, \swarrow)}
\end{aligned}
$$

Thus Formula (3.4.8) provides us with a new description of the measure $\mathbf{W}_{g}^{(-\alpha)}$.

Proof of Theorem 3.4. Of course, from (3.4.3), and (3.4.5), it suffices to show (3.4.6). Note that, from $(3.3 .8)$, for $\Gamma_{t} \in b\left(\mathcal{F}_{t}\right)$, one has :

$$
\mathbf{W}^{(-\alpha)}\left(\Gamma_{t} 1_{g \leq t}\right)=P_{0}^{(-\alpha)}\left(\Gamma_{t} R_{t}^{2 \alpha}\right)
$$

Thus, for every $s \leq t$ and $\Gamma_{s} \in b\left(\mathcal{F}_{s}\right)$, since $\left(R_{t}^{2 \alpha}-L_{t}, t \geq 0\right)$ is a martingale (see (3.3.3)), we have :

$$
\begin{aligned}
\mathbf{W}^{(-\alpha)}\left(\Gamma_{s} 1_{s \leq g \leq t}\right) & =P_{0}^{(-\alpha)}\left(\Gamma_{s}\left(R_{t}^{2 \alpha}-R_{s}^{2 \alpha}\right)\right) \\
& =P_{0}^{(-\alpha)}\left(\Gamma_{s}\left(L_{t}-L_{s}\right)\right)
\end{aligned}
$$


We deduce from the monotone class theorem and from (3.4.10) that, for every positive previsible process $\left(\Phi_{u}, u \geq 0\right)$, one has :

$$
\begin{aligned}
\mathbf{W}^{(-\alpha)}\left(\Phi_{g}\right) & =P_{0}^{(-\alpha)}\left(\int_{0}^{\infty} \Phi_{u} d L_{u}\right) \\
& =\int_{0}^{\infty} P_{0}^{(-\alpha)}\left(\Phi_{u} \mid R_{u}=0\right) P_{0}^{(-\alpha)}\left(d L_{u}\right) \\
& =\int_{0}^{\infty} \Pi^{(-\alpha, u)}\left(\Phi_{u}\right) \frac{\alpha 2^{\alpha} u^{\alpha-1}}{\Gamma(1-\alpha)} d u
\end{aligned}
$$

from (3.3.6). Hence :

$$
\begin{aligned}
\mathbf{W}^{(-\alpha)}\left(\Phi_{g}\right)= & \left(\int_{0}^{\infty} \Pi_{0}^{(-\alpha, u)} \frac{\alpha 2^{\alpha} u^{\alpha-1}}{\Gamma(1-\alpha)} d u\right)\left(\Phi_{g}\right) \\
= & \left(2 \alpha \int_{0}^{\infty} d u \frac{2^{-\frac{\delta}{2}}}{\Gamma\left(\frac{\delta}{2}\right)} u^{-\frac{\delta}{2}} \Pi_{0}^{\left(\frac{\delta}{2}-1, u\right)}\right)\left(\Phi_{g}\right) \\
& (\operatorname{with} \delta=2(1-\alpha)) \\
= & 2 \alpha \boldsymbol{\Lambda}_{0,0}^{(2(1-\alpha))}\left(\Phi_{g}\right) \quad \text { from }(3.4 .3)
\end{aligned}
$$

3.4.4 In the general framework of linear diffusions, formulae (3.4.7) and (3.4.8) become :

$$
\mathbf{W}_{g}^{*}=\int_{0}^{\infty} d t p(t, 0,0) \Pi_{0}^{(t)}
$$

(This is formula (3.2.7)) and :

$$
\mathbf{W}_{g}^{*}=\int_{0}^{\infty} P_{0}^{(m, \swarrow)} d S(m)
$$

The reader may refer to ([PY2], 2.2, Corollary 2.1, p. 298) where the probability $P_{0}^{(m, \swarrow}$ ) is defined in terms of the law $P_{0}^{\uparrow}$ (of the process $\left(X_{t}, t \geq 0\right.$ ) conditioned to remain $>0$ ) just as $P_{0}^{(-\alpha, m, \swarrow)}$ is, in terms of the law $P_{0}^{(\alpha)}$. (see (3.4.4)) with $\delta=2(1+\alpha)$ ).

\subsection{Penalisations of $\alpha$-stable symmetric process.}

In this subsection, we summarize a work in progress by K. Yano, Y. Yano and M. Yor [YYY] which bears upon the penalisation of the $\alpha$-stable symmetric Lévy process, with $1<\alpha \leq 2$. This summary is not exhaustive ; rather, it is an invitation to read [YYY].

3.5.1 Notation and classical results. (see, e.g., [Ber], [Ch], [SY])

3.5.1.1 $\left(\Omega,\left(X_{t}, \mathcal{F}_{t}\right)_{t \geq 0}, \mathcal{F}_{\infty}, P_{x}, x \in \mathbb{R}\right)$ denote the canonical realisation of the $\alpha$-stable symmetric Lévy process, with $1<\alpha \leq 2$. The notations are the same as in 1.0.1, with the difference that $\Omega$ now denotes the space of càdlàg functions from $\mathbb{R}_{+}$to $\mathbb{R}$. $\alpha$ being fixed once and for all, the dependency in $\alpha$ will be mostly omitted in our notation. This Lévy process $\left(X_{t}, t \geq 0\right)$ is characterised via :

$$
E_{0}\left(e^{i \lambda X_{t}}\right)=\exp \left(-t|\lambda|^{\alpha}\right) \quad(t \geq 0, \lambda \in \mathbb{R})
$$

The case $\alpha=2$ corresponds to $\left(X_{t}, t \geq 0\right) \equiv\left(B_{2 t}, t \geq 0\right)$ where $\left(B_{t}, t \geq 0\right)$ is a standard 1-dimensional Brownian motion. 
3.5.1.2 $p_{t}(x)$ denotes the density (with respect to Lebesgue measure on $\mathbb{R}$ ) of the r.v. $X_{t}$ and $u_{\lambda}(\lambda>0)$ the resolent kernel :

$$
\begin{gathered}
P_{x}\left(X_{t} \in d y\right)=p_{t}(x-y) d y \\
p_{t}(0)=\frac{1}{\alpha \pi} \Gamma\left(\frac{1}{\alpha}\right) t^{-\frac{1}{\alpha}} \quad(t>0) \\
u_{\lambda}(x):=\int_{0}^{\infty} e^{-\lambda t} p_{t}(x) d t=\frac{1}{\pi} \int_{0}^{\infty} \frac{\cos (x y)}{\lambda+y^{\alpha}} d y \\
u_{\lambda}(0)=\frac{1}{\pi} B\left(1-\frac{1}{\alpha}, \frac{1}{\alpha}\right) \lambda^{\frac{1}{\alpha}-1}
\end{gathered}
$$

Let, for every $a \in \mathbb{R}, T_{a}:=\inf \left\{t \geq 0 ; X_{t}=a\right\}$. Then :

$$
E_{x}\left[e^{-\lambda T_{0}}\right]=\frac{u_{\lambda}(x)}{u_{\lambda}(0)}
$$

3.5.1.3 We denote by $\left(L_{t}^{x}, t \geq 0, x \in \mathbb{R}\right)$ the jointly continuous process of local times of $\left(X_{t}, t \geq 0\right) \cdot\left(L_{t}, t \geq 0\right)$ stands for $\left(L_{t}^{0}, t \geq 0\right)$, the process of local time at 0 , and $\left(\tau_{l}, l \geq 0\right)$ its right continuours inverse. We have :

$$
E_{0}\left(e^{-\lambda \tau_{l}}\right)=\exp \left(-\frac{l}{u_{\lambda}(0)}\right)
$$

so that, from $(3.5 .5),\left(\tau_{l}, l \geq 0\right)$ is a stable subordinator with index $1-\frac{1}{\alpha}$. On the other hand :

$$
\begin{aligned}
E_{0}\left(\int_{0}^{\infty} e^{-\lambda t} d L_{t}\right) & =\int_{0}^{\infty} E_{0}\left(e^{-\lambda \tau_{l}}\right) d l=u_{\lambda}(0) \\
\text { and } \quad E_{0}\left(d L_{t}\right) & =p_{t}(0) d t=\frac{1}{\alpha \pi} \Gamma\left(\frac{1}{\alpha}\right) t^{-\frac{1}{\alpha}} d t
\end{aligned}
$$

More generally : $E_{x}\left(d L_{t}\right)=E_{0}\left(d_{t} L_{t}^{x}\right)=p_{t}(x) d t$

3.5.1.4 We denote by $h$ the function defined by :

$$
h(x):=\frac{1}{2 \cos \left[\left(1-\frac{\alpha}{2}\right) \pi\right]}|x|^{\alpha-1} \quad(x \in \mathbb{R})
$$

This function is harmonic for the process $\left(X_{t}, t \geq 0\right)$ killed when it reaches 0 , i.e. : for every $x \in \mathbb{R}$, and $t \geq 0$ :

$$
E_{x}\left[h\left(X_{t}\right) 1_{T_{0} \geq t}\right]=h(x)
$$

Moreover, there exists a constant $c>0$ such that, for every $x \in \mathbb{R}$ :

$$
\left(N_{t}^{x}:=h\left(X_{t}\right)-h(x)-c L_{t}^{x}, t \geq 0\right)
$$

is a square integrable $P_{x}$-martingale (this formula may compared with (3.1.7)) .

3.5.1.5 Since 0 is a regular and recurrent point for $\left(X_{t}, t \geq 0\right)$, Itô's excursion theory may be applied. We denote as $\widetilde{\Omega}$ the excursions space, where $\left(Y_{t}, t \geq 0\right)$ is the process of coordinates, 
$\xi$ the lifetime of the generic excursions and $n$ Itô's excursion measure. The master formula implies :

$$
E_{0}\left[\int_{0}^{\infty} e^{-\lambda t} f\left(X_{t}\right) d t\right]=E_{0}\left(\int_{0}^{\infty} e^{-\lambda \tau_{l}} d l\right) \cdot \int_{0}^{\infty} e^{-\lambda t} n\left(f\left(Y_{t}\right)\right) d t
$$

for any $f: \mathbb{R} \rightarrow \mathbb{R}_{+}$Borel, such that $f(0)=0$. In particular :

$$
n(\xi>t)=\frac{\alpha \pi}{B\left(1-\frac{1}{\alpha}, \frac{1}{\alpha}\right) \Gamma\left(\frac{1}{\alpha}\right)} t^{\frac{1}{\alpha}-1}
$$

There exist a function $\rho(t, x)$ which is positive and jointly measurable such that :

$$
\begin{aligned}
n\left(Y_{t} \in d x\right) & =\rho(t, x) d x \\
\text { and } \quad P_{x}\left(T_{0} \in d t\right) & =\rho(t, x) d t
\end{aligned}
$$

\subsubsection{Definition of the $\sigma$-finite measure $\mathbf{P}$.}

The measure $\mathbf{P}$ is define on $\left(\Omega, \mathcal{F}_{\infty}\right)$ by :

$$
\begin{aligned}
\mathbf{P} & :=\int_{0}^{\infty} P_{0}\left(d L_{u}\right) Q^{(u)} \circ P_{0}^{\uparrow} \\
& =\frac{1}{\alpha \pi} \Gamma\left(\frac{1}{\alpha}\right) \int_{0}^{\infty} u^{-\frac{1}{\alpha}} Q^{(u)} \circ P_{0}^{\uparrow}
\end{aligned}
$$

(from (3.5.3)). We now explain the notations in (3.5.18) :

- $Q^{(u)}$ denotes the law fo the $\alpha$-stable symmetric bridge with length $u$ :

$$
Q^{(u)}\left(\Gamma_{u}\right)=P_{0}\left(\Gamma_{u} \mid X_{u}=0\right) \quad\left(\Gamma_{u} \in \mathcal{F}_{u}\right)
$$

- We denote by $\left(P_{x}^{0}, x \neq 0\right)$ the law of the process $\left(X_{t}, t \geq 0\right)$ starting from $x$ and killed in $T_{0}$ :

$$
P_{x}^{0}\left(\Gamma_{t}\right)=E_{x}\left(\Gamma_{t} 1_{T_{0}>t}\right) \quad \Gamma_{t} \in b\left(\mathcal{F}_{t}\right)
$$

and by $P_{x}^{\uparrow}$ the law obtained from that of $P_{x}^{0}$ by Doob's $h$-transform (recall that $h$ is defined by (3.5.11) and that it is harmonic for the process $\left(X_{t}, t \geq 0\right)$ killed in $\left.T_{0}\right)$ :

$$
\left.P_{x}^{\uparrow}\right|_{\mathcal{F}_{t}}:=\left.\frac{h\left(X_{t}\right)}{h(x)} \cdot P_{x}^{0}\right|_{\mathcal{F}_{t}} \quad x \neq 0
$$

Letting $x$ tend to 0 in (3.5.21), we obtain :

$$
\left.P_{0}^{\uparrow}\right|_{\mathcal{F}_{t}}:=\left.\lim _{x \rightarrow 0} \frac{h\left(X_{t}\right)}{h(x)} \cdot P_{x}^{0}\right|_{\mathcal{F}_{t}}=\left.h\left(X_{t}\right) n\right|_{\mathcal{F}_{t}}
$$

- Another manner to define $P_{0}^{\uparrow}$ consists to first define the law $M^{(t)}$ of the stable meander (with duration $t$ ) :

$$
M^{(t)}\left(\Gamma_{t}\right):=n\left(\Gamma_{t} \mid \xi>t\right)=\frac{n\left(\Gamma_{t} \cap(\xi>t)\right)}{n(\xi>t)} \quad\left(\Gamma_{t} \in b\left(\mathcal{F}_{t}\right)\right)
$$


then to show that :

$$
M^{(t)} \underset{t \rightarrow \infty}{\longrightarrow} P_{0}^{\uparrow}
$$

with the preceding convergence taking place along $\left(\mathcal{F}_{s}\right)$, i.e. : for every $s \geq 0$ and $\Gamma_{s} \in b\left(\mathcal{F}_{s}\right):$

$$
M^{(t)}\left(\Gamma_{s}\right) \underset{t \rightarrow \infty}{\longrightarrow} P_{0}^{\uparrow}\left(\Gamma_{s}\right)
$$

- The measure $\mathbf{P}$ defined by (3.5.18) plays for the symmetric $\alpha$-stable Lévy process the same role as the measure $\mathbf{W}$ for standard Brownian motion. Indeed, for $\alpha=2$, (3.5.18) becomes

$$
\mathbf{P}=\frac{1}{2 \sqrt{\pi}} \int_{0}^{\infty} \frac{1}{\sqrt{u}} Q^{(u)} \circ P_{0}^{\uparrow}=\frac{1}{\sqrt{2}} \mathbf{W}
$$

where $\mathbf{W}$ is defined by (1.1.43). The multiplication factor $\frac{1}{\sqrt{2}}$ arises from the fact that, for $\alpha=2$, the 2 -stable symmetric Lévy process $\left(X_{t}, t \geq 0\right)$ is the process $\left(B_{2 t}, t \geq 0\right)$ and not $\left(B_{t}, t \geq 0\right)$ (see (3.5.1)).

3.5.3 The martingales $\left(M_{t}(F), t \geq 0\right)\left(F \in L_{+}^{1}(\Omega, \mathbf{P})\right)$ associated to $\mathbf{P}$.

3.5.3.1 In the same manner that we have associated to the $\sigma$-finite measures $\mathbf{W}, \mathbf{W}^{(2)}$ and $\mathbf{W}^{*}$ introduced in Section 1,2 and (3.2.2.3), a familly of martingales, we associate here to every r.v. $F \in L_{+}^{1}\left(\Omega, \mathcal{F}_{\infty}, \mathbf{P}\right)$ the $\left(\left(\mathcal{F}_{t}\right)_{t \geq 0}, P_{0}\right)$ martingale $\left(M_{t}(F), t \geq 0\right)$ characterized by : for any $t \geq 0$ and $\Gamma_{t} \in b\left(\mathcal{F}_{t}\right)$ :

$$
E_{\mathbf{P}}\left[F \cdot \Gamma_{t}\right]=E_{0}\left(M_{t}(F) \cdot \Gamma_{t}\right)
$$

In particular, for every $t \geq 0$ :

$$
E_{0}\left[M_{t}(F)\right]=E_{\mathbf{P}}(F)
$$

3.5.3.2 Example 1. Let $f: \mathbb{R}_{+} \rightarrow \mathbb{R}_{+}$Borel such that $\int_{0}^{\infty} f(y) d y<\infty$. Then :

$$
M_{t}\left(f\left(L_{\infty}\right)\right)=f\left(L_{t}\right) h\left(X_{t}\right)+\int_{L_{t}}^{\infty} f(x) d x \quad(t \geq 0)
$$

where, in (3.5.28), the function $h$ is defined by (3.5.11). It is not difficult to see, thanks to (3.5.13), that $\left(M_{t}\left(f\left(L_{\infty}\right)\right), t \geq 0\right)$ defined by (3.5.28) is indeed a martingale. We shall also denote the analogy between (3.5.28) with formula (3.2.21) obtained in the framework of linear diffusions :

$$
M_{t}^{*}\left(f\left(L_{\infty}\right)\right)=f\left(L_{t}\right) S\left(X_{t}\right)+\int_{L_{t}}^{\infty} f(y) d y
$$

Thus, we shift from (3.5.29) to (3.5.28) by replacing simply the scale function $S$ by the function $h$ (these two functions are such that, in both cases, $\left(S\left(X_{t}\right) 1_{t<T_{0}}, t \geq 0\right)$ and $\left(h\left(X_{t}\right) 1_{t<T_{0}}\right)$ are martingales).

3.5.3.3 Example 2. (Feynman-Kac martingales) Let $q$ denote a Radon measure on $\mathbb{R}$ such that :

$$
0<\int_{\mathbb{R}}(1+h(x)) q(d x)<\infty \quad \text { with } h \text { defined by (3.5.11) }
$$


Let

$$
A_{t}^{(q)}:=\int_{\mathbb{R}} L_{t}^{x} q(d x)
$$

and $A_{\infty}^{(q)}:=\lim _{t \rightarrow \infty} A_{t}^{(q)}$. Then

$$
M_{t}\left(\exp \left(-A_{\infty}^{(q)}\right)\right)=\varphi_{q}\left(X_{t}\right) \cdot \exp \left(-A_{t}^{(q)}\right)
$$

with

$$
\varphi_{q}(x):=\lim _{t \rightarrow \infty} \frac{E_{x}\left(\exp -A_{t}^{(q)}\right)}{n(\xi>t)} \quad(x \in \mathbb{R})
$$

We note that : $E_{\mathbf{P}}\left(\exp \left(-A_{\infty}^{(q)}\right)\right)=\varphi_{q}(0)$.

Other description of the function $\varphi_{q}$ are found in [YYY]. The reader will have noticed the complete analogy between the definition of $M_{t}\left(\exp -A_{\infty}^{(q)}\right)$ given by (3.5.32) and that, in the Brownian case, of $M_{t}\left(\exp -A_{\infty}^{(q)}\right)$ which is given by (1.2.19) :

$$
M_{t}\left(\exp -\frac{1}{2} A_{\infty}^{(q)}\right)=\varphi_{q}\left(X_{t}\right) \exp \left(-\frac{1}{2} A_{t}^{(q)}\right)
$$

\subsubsection{Relations between $P$ and penalisations}

Penalisaton theorems, which we now present, are found in [YYY] :

Theorem 3.5.1 Let $f: \mathbb{R}_{+} \rightarrow \mathbb{R}_{+}$Borel such that $\int_{0}^{\infty} f(y) d y<\infty$. Then :

1) For every $s \geq 0, \Gamma_{s} \in b\left(\mathcal{F}_{s}\right)$ :

$$
\lim _{t \rightarrow \infty} \frac{E_{0}\left[\Gamma_{s} f\left(L_{t}\right)\right]}{n(\xi>t)}=E_{0}\left[\Gamma_{s} M_{s}\left(f\left(L_{\infty}\right)\right)\right]
$$

where $\left(M_{t}\left(f\left(L_{\infty}\right), t \geq 0\right)\right.$ is the positive martingale defined by (3.5.28).

2) Let $P_{0, \infty}^{f(L)}$ the probability induced on $\left(\Omega, \mathcal{F}_{\infty}\right)$ by :

$$
\left.P_{0, \infty}^{f(L)}\right|_{\mathcal{F}_{t}}:=\left.\frac{M_{t}\left(f\left(L_{\infty}\right)\right)}{E_{0}\left(M_{0}\left(f\left(L_{\infty}\right)\right)\right.} \cdot P_{0}\right|_{\mathcal{F}_{t}}
$$

Then, the absolute continuity formula :

$$
f\left(L_{\infty} \cdot \mathbf{P}=E_{\mathbf{P}}\left(f\left(L_{\infty}\right)\right) \cdot P_{0, \infty}^{f(L)}\right. \text { holds }
$$

(Note that: $E_{\mathbf{P}}\left(f\left(L_{\infty}\right)\right)=\int_{0}^{\infty} f(y) d y=E_{0}\left(M_{t}\left(f\left(L_{\infty}\right)\right)\right.$.)

Clearly, this formula (3.5.36) is formally identical to formula (1.1.107) obtained in the Brownian set-up (with $h^{+}=h^{-}=f$ ).

Theorem 3.5.2 Let $q$ denote a Radon measure on $\mathbb{R}$ such that $0<\int_{\mathbb{R}}(1+h(x)) q(d x)<\infty$ (with $h$ defined by (3.5.11)) and let $A_{t}^{(q)}:=\int_{\mathbb{R}} L_{t}^{x} q(d x)$. Then :

1) For every $s \geq 0$ and $\Gamma_{s} \in b\left(\mathcal{F}_{s}\right)$ :

$$
\lim _{t \rightarrow \infty} \frac{E_{0}\left(\Gamma_{s} \exp \left(-A_{t}^{(q)}\right)\right)}{n(\xi>t)}=E_{0}\left[\Gamma_{s} M_{s}\left(\exp \left(-A_{\infty}^{(q)}\right)\right)\right]
$$


where $\left(M_{t}\left(\exp \left(-A_{\infty}^{(q)}\right)\right), t \geq 0\right)$ is the positive martingale defined by (3.5.32).

2) Let $P_{0, \infty}^{(q)}$ denote the probability induced on $\left(\Omega, \mathcal{F}_{\infty}\right)$ by :

$$
\left.P_{0, \infty}^{(q)}\right|_{\mathcal{F}_{t}}=\left.\frac{M_{t}\left(\exp \left(-A_{\infty}^{(q)}\right)\right)}{E_{0}\left(M_{0}\left(\exp -\left(A_{\infty}^{(q)}\right)\right)\right.} \cdot P_{0}\right|_{\mathcal{F}_{t}}
$$

Then, the absolute continuity formula :

$$
\exp \left(-A_{\infty}^{(q)}\right) \cdot \mathbf{P}=E_{\mathbf{P}}\left(\exp \left(-A_{\infty}^{(q)}\right)\right) \cdot P_{0, \infty}^{(q)} \quad \text { holds }
$$

Of course, this formula is formally idendical to formula (1.1.16) obtained in the Brownian framework (one should note that $E_{\mathbf{P}}\left(\exp -A_{\infty}^{(q)}\right)=\varphi_{q}(0)=E_{0}\left(M_{t}\left(\exp \left(-A_{\infty}^{(q)}\right)\right)\right.$ where $\varphi_{q}$ is defined by (3.5.33).

Throughout the preceding, a particular role was played by point $x=0$. However, since the $\alpha$-stable symmetric Lévy process is invariant by translation, we may define, for every $x \in \mathbb{R}$ the $\sigma$-finite measure $\mathbf{P}_{x}$ by the formula:

$$
E_{\mathbf{P}_{x}}\left[F\left(X_{t}, t \geq 0\right)\right]=E_{\mathbf{P}}\left[F\left(x+X_{t}, t \geq 0\right)\right]
$$

for every positive measurable functional ; thus, the knowledge of $\mathbf{P}$ induces that of $\mathbf{P}_{x}$, for any $x \neq 0$.

The reader will have noticed the quasi complete analogy between, on one hand, the results of [YYY] which we just described in the set-up of the $\alpha$-stable symmetric Lévy process with $1<\alpha \leq 2$ and the results of Chapter 1 of this monograph, in the Brownian set-up. We refer the interested reader to [YYY] where the proofs of the results announced above are found, as well as many other informations. 


\section{Chapter 4. An analogue of $\mathrm{W}$ for discrete Markov chains. 4.0 Introduction.}

In this chapter, we construct for discrete Markov processes some $\sigma$-finite measures which enjoy similar properties as the measure $\mathbf{W}$ studied in Chapter 1 . Very informally, these $\sigma$-finite measures are obtained by "conditioning a recurrent Markov process to be transient".

Our construction applies to discrete versions of one and two-dimensional Brownian motion, i.e. simple random walk on $\mathbb{Z}$ and $\mathbb{Z}^{2}$, but it can also be applied to a much larger class of Markov chains.

Unfortunately, we are not able at this point to generalize our construction to continuous Markov processes, although this generalization seems to be possible.

This chapter is divided into three sections; in Section 4.1, we give the construction of the $\sigma$-finite measures mentioned above ; in Section 4.2, we study the main properties of these measures, and in Section 4.3, we study some examples in more details.

\subsection{Construction of the $\sigma$-finite measures $\mathrm{W}$}

4.1.1 Notation and hypothesis.

Let $E \overline{\text { be a countable set, }\left(X_{n}\right)_{n} \geq 0}$ the canonical process on $E^{\mathbb{N}},\left(\mathcal{F}_{n}\right)_{n \geq 0}$ its natural filtration, and $\mathcal{F}_{\infty}$ the $\sigma$-field generated by $\left(X_{n}\right)_{n \geq 0}$.

Let us denote by $\left(\mathbb{P}_{x}\right)_{x \in E}$ the family of probability measures on $\left(E^{\mathbb{N}},\left(\mathcal{F}_{n}\right)_{n \geq 0}, \mathcal{F}\right)$ associated to a Markov process $\left(\mathbb{E}_{x}\right.$ below denotes the expectation with respect to $\left.\mathbb{P}_{x}\right) ;$ more precisely, we suppose there exist probability transitions $\left(p_{y, z}\right)_{y, z \in E}$ such that:

$$
\mathbb{P}_{x}\left(X_{0}=x_{0}, X_{1}=x_{1}, \ldots, X_{k}=x_{k}\right)=\mathbf{1}_{x_{0}=x} p_{x_{0}, x_{1}} p_{x_{1}, x_{2}} \ldots p_{x_{k-1}, x_{k}}
$$

for all $k \geq 0, x_{0}, x_{1}, \ldots, x_{k} \in E$.

We assume three more hypotheses :

- For all $x \in E$, the set of $y \in E$ such that $p_{x, y}>0$ is finite (i.e. the graph associated to the Markov chain is locally finite).

- For all $x \in E$, the canonical process is recurrent under the probability $\mathbb{P}_{x}$.

- For all $x, y \in E$, there exists $n \in \mathbb{N}$ such that $\mathbb{P}_{x}\left(X_{n}=y\right)>0$ (i.e. the graph of the Markov chain is connected).

4.1.2 A family of new probabilities.

From the family of probabilities $\left(\mathbb{P}_{x}\right)_{x \in E}$, we will construct families of $\sigma$-finite measures which should be informally considered to be the law of $\left(X_{n}\right)_{n \geq 0}$ under $\mathbb{P}_{x}$, after conditionning this process to be transient.

More precisely, let us fix a point $x_{0} \in E$ and let us suppose there exists a function $\phi: E \rightarrow \mathbb{R}_{+}$ such that :

- $\phi(x) \geq 0$ for all $x \in E$, and $\phi\left(x_{0}\right)=0$.

- $\phi$ is harmonic with respect to $\mathbb{P}$, except at the point $x_{0}$, i.e. for all $x \neq x_{0}, \sum_{y \in E} p_{x, y} \phi(y)=\mathbb{E}_{x}\left[\phi\left(X_{1}\right)\right]=\phi(x)$. 
Generally, $\phi$ is not unique, but it will fixed in this section. For any $r \in(0,1)$, let us define :

$$
\psi_{r}(x)=\frac{r}{1-r} \mathbb{E}_{x_{0}}\left[\phi\left(X_{1}\right)\right]+\phi(x) .
$$

From this definition, the following properties hold :

- For all $x \neq x_{0}, \psi_{r}(x)=\mathbb{E}_{x}\left[\psi_{r}\left(X_{1}\right)\right]$.

- $\psi_{r}\left(x_{0}\right)=r \mathbb{E}_{x_{0}}\left[\psi_{r}\left(X_{1}\right)\right]$

Now, for $y \in E$ and $k \geq-1$, let us denote by $L_{k}^{y}$ the local time of $X$ at point $y$ and time $k$, i.e. :

$$
L_{k}^{y}=\sum_{m=0}^{k} \mathbf{1}_{X_{m}=y}
$$

(in particular, $L_{-1}^{y}=0$ and $L_{0}^{y}=\mathbf{1}_{X_{0}=y}$ ). The properties of $\psi_{r}$ imply the following result :

Proposition 4.1.1 For all $x \in E,\left(\psi_{r}\left(X_{n}\right) r^{L_{n-1}^{x_{0}}}\right)_{n \geq 0}$ is a martingale under $\mathbb{P}_{x}$.

Proof of Proposition 4.1.1 For every $n \geq 0$, by Markov property :

$$
\begin{aligned}
\mathbb{E}_{x}\left[\psi_{r}\left(X_{n+1}\right) r^{L_{n}^{x_{0}}} \mid \mathcal{F}_{n}\right] & =r^{L_{n}^{x_{0}}} \mathbb{E}_{x}\left[\psi_{r}\left(X_{n+1}\right) \mid \mathcal{F}_{n}\right] \\
& =r^{L_{n}^{x_{0}}} \psi_{r}\left(X_{n}\right)\left(\mathbf{1}_{X_{n} \neq x_{0}}+\frac{1}{r} \mathbf{1}_{X_{n}=x_{0}}\right)=r^{L_{n-1}^{x_{0}}} \psi_{r}\left(X_{n}\right) .
\end{aligned}
$$

Corollary 4.1.2 (from (4.1.3) and (4.1.4))

There exists a finite measure $\mu_{x}^{(r)}$ on $\left(E^{\mathbb{N}}, \mathcal{F}_{\infty}\right)$ such that :

$$
\mu_{x \mid \mathcal{F}_{n}}^{(r)}=\psi_{r}\left(X_{n}\right) r^{L_{n-1}^{x_{0}}} \cdot \mathbb{P}_{x \mid \mathcal{F}_{n}}
$$

At this point, we remark that, for all $\sigma, 0<\sigma<1 / r$ :

- $\psi_{r}(x) \leq \sup \left(\frac{1-\sigma r}{\sigma(1-r)}, 1\right) \cdot \psi_{\sigma r}(x)$ for all $x \in E$.

- Consequently, for $n \geq 1$ :

$$
\begin{aligned}
& \mu_{x}^{(r)}\left(\sigma^{L_{n-1}^{x_{0}}}\right)=\mathbb{P}_{x}\left[\psi_{r}\left(X_{n}\right)(r \sigma)^{\left.L_{n-1}^{x_{0}}\right]} \quad(\text { from }(4.1 .7))\right. \\
& \leq \sup \left(\frac{1-\sigma r}{\sigma(1-r)}, 1\right) \mathbb{P}_{x}\left[\psi_{\sigma r}\left(X_{n}\right)(r \sigma)^{\left.L_{n-1}^{x_{0}}\right]}\right. \\
& \leq \sup \left(\frac{1-\sigma r}{\sigma(1-r)}, 1\right) \mu_{x}^{(\sigma r)}(1)=C
\end{aligned}
$$

where $C<\infty$ does not depend on $n$.

Therefore, $\mu_{x}^{(r)}\left(\sigma^{L_{0}^{x_{0}}}\right)<\infty$; in particular, $L_{\infty}^{x_{0}}<\infty, \mu_{x}^{(r)}$-a.s. It is now possible to define a measure $\mathbb{Q}_{x}^{(r)}$, by : $\mathbb{Q}_{x}^{(r)}=\left(\frac{1}{r}\right)^{L_{\infty}^{x_{0}}} \cdot \mu_{x}^{(r)}$; this measure is $\sigma$-finite since the sets $\left\{L_{\infty}^{x_{0}} \leq m\right\}$ increase to $\left\{L_{\infty}^{x_{0}}<\infty\right\}$; moreover $L_{\infty}^{x_{0}}=\infty$ is $\mathbb{Q}_{x}^{(r)}$-negligible, and

$$
\mathbb{Q}_{x}^{(r)}\left(L_{\infty}^{x_{0}} \leq m\right) \leq\left(\frac{1}{r}\right)^{m} \mu_{x}^{(r)}(1)<\infty
$$


4.1.3 Definition of the measures $\left(\mathbb{Q}_{x}, x \in E\right)$.

Here is a remarkable result, which explains the interest of this construction :

Theorem 4.1.3 The two following properties hold:

(i) For all $x \in E, \mathbb{Q}_{x}^{(r)}$ does not depend on $r \in(0,1)$.

(ii) Let $\mathbb{Q}_{x}$ denote the measure equal to $\mathbb{Q}_{x}^{(r)}$ for all $r \in(0,1)$, and $F_{n} \geq 0$ a $\mathcal{F}_{n}$-measurable functional. If $q$ is a function from $E$ to $[0,1]$, such that $\{q<1\}$ is a finite set, then:

$$
\mathbb{Q}_{x}\left[F_{n} \prod_{k=0}^{\infty} q\left(X_{k}\right)\right]=\mathbb{E}_{x}\left[F_{n} \psi_{q}\left(X_{n}\right) \prod_{k=0}^{n-1} q\left(X_{k}\right)\right]
$$

$$
\text { where for } y \in E, \psi_{q}(y)=\mathbb{Q}_{y}\left[\prod_{k=0}^{\infty} q\left(X_{k}\right)\right] \text {. }
$$

Remark 4.1.4 If we denote by $\mu_{x}^{(q)}$ the measure defined by :

$$
\mu_{x}^{(q)}=\prod_{k=0}^{\infty} q\left(X_{k}\right) \cdot \mathbb{Q}_{x}
$$

we obtain :

$$
\mu_{x \mid \mathcal{F}_{n}}^{(q)}=\psi_{q}\left(X_{n}\right) \prod_{k=0}^{n-1} q\left(X_{k}\right) \cdot \mathbb{P}_{x \mid \mathcal{F}_{n}} .
$$

These relations are similar to relations between $\mathbf{W}$ and Feynman-Kac penalisations of Wiener measure $W$ (see Chap. 1, Th. 1.1.2, formulae (1.1.7), (1.1.8), (1.1.16)).

Moreover, $\psi_{q}$ satisfies the "Sturm-Liouville equation" :

$$
\psi_{q}(x)=q(x) \mathbb{E}_{x}\left[\psi_{q}\left(X_{1}\right)\right]
$$

Proof of Theorem 4.1.3 To begin with, let us prove the point ii) (with $\mathbb{Q}_{x}^{(r)}$ instead of $\left.\mathbb{Q}_{x}\right)$ for a function $q$ such that $q\left(x_{0}\right)<1$. Under the hypotheses of Theorem 4.1.3, for all $n \geq 0, F_{n} \prod_{k=0}^{N-1} q\left(X_{k}\right)\left(\frac{1}{r}\right)^{L_{N-1}^{x_{0}}}$ tends to $F_{n} \prod_{k=0}^{\infty} q\left(X_{k}\right)\left(\frac{1}{r}\right)^{L_{\infty}^{x_{0}}}$ as $N \rightarrow \infty$ and is dominated by $\left(\frac{q\left(x_{0}\right)}{r} \vee 1\right)^{L_{\infty}^{x_{0}}}$, which is $\mu_{x}^{(r)}$-integrable because $\frac{q_{0}}{r} \vee 1<\frac{1}{r}$.

By dominated convergence, if for $y \in E, k \geq 0$, we define :

$$
\psi_{q}^{r, k}(y):=\mathbb{E}_{y}\left[\psi_{r}\left(X_{k}\right) \prod_{m=0}^{k-1} q\left(X_{m}\right)\right]
$$

for all $x \in E$ :

$$
\begin{aligned}
& \mathbb{E}_{x}\left[F_{n} \psi_{q}^{r, N-n}\left(X_{n}\right) \prod_{k=0}^{n-1} q\left(X_{k}\right)\right]=\mathbb{E}_{x}\left[F_{n} \psi_{r}\left(X_{N}\right) \prod_{k=0}^{N-1} q\left(X_{k}\right)\right] \\
& =\mu_{x}^{(r)}\left[F_{n} \prod_{k=0}^{N-1} q\left(X_{k}\right)\left(\frac{1}{r}\right)^{L_{N-1}^{x_{0}}}\right] \\
& \underset{N \rightarrow \infty}{\rightarrow} \mu_{x}^{(r)}\left[F_{n} \prod_{k=0}^{\infty} q\left(X_{k}\right)\left(\frac{1}{r}\right)^{L_{\infty}^{x_{0}}}\right]=\mathbb{Q}_{x}^{(r)}\left[F_{n} \prod_{k=0}^{\infty} q\left(X_{k}\right)\right] .
\end{aligned}
$$


In particular, if we take $n=0$ and $F_{0}=1$ :

$$
\psi_{q}^{r, N}(y) \underset{N \rightarrow \infty}{\rightarrow} \mathbb{Q}_{y}^{(r)}\left[\prod_{k=0}^{\infty} q\left(X_{k}\right)\right]
$$

for all $y \in E$.

Moreover :

$$
\begin{aligned}
& \psi_{q}^{r, N-n}(y) \leq \mathbb{E}_{y}\left[\left(q\left(x_{0}\right)\right)^{L_{N-n-1}^{x_{0}}} \psi_{r}\left(X_{N-n}\right)\right] \\
& \leq \sup \left(\frac{r}{q\left(x_{0}\right)}\left(\frac{1-q\left(x_{0}\right)}{1-r}\right), 1\right) \mathbb{E}_{y}\left[\left(q\left(x_{0}\right)\right)^{L_{N-n-1}^{x_{0}}} \psi_{q\left(x_{0}\right)}\left(X_{N-n}\right)\right] \\
& =\sup \left(\frac{r}{q\left(x_{0}\right)}\left(\frac{1-q\left(x_{0}\right)}{1-r}\right), 1\right) \psi_{q\left(x_{0}\right)}(y)
\end{aligned}
$$

where

$$
\begin{aligned}
\mathbb{E}_{x}\left[\psi_{q\left(x_{0}\right)}\left(X_{n}\right) \prod_{k=0}^{n-1} q\left(X_{k}\right)\right] & \leq \mathbb{E}_{x}\left[\psi_{q\left(x_{0}\right)}\left(X_{n}\right)\left(q\left(x_{0}\right)\right)^{L_{n-1}^{x_{0}}}\right] \\
& =\psi_{q\left(x_{0}\right)}(x)<\infty
\end{aligned}
$$

By dominated convergence :

$$
\mathbb{E}_{x}\left[F_{n} \psi_{q}^{r, N-n}\left(X_{n}\right) \prod_{k=0}^{n-1} q\left(X_{k}\right)\right] \underset{N \rightarrow \infty}{\rightarrow} \mathbb{E}_{x}\left[F_{n} \psi_{q}^{(r)}\left(X_{n}\right) \prod_{k=0}^{n-1} q\left(X_{k}\right)\right],
$$

where $\psi_{q}^{(r)}(y)=\mathbb{Q}_{y}^{(r)}\left[\prod_{k=0}^{\infty} q\left(X_{k}\right)\right]$.

The two previous limits are equal; therefore :

$$
\mathbb{Q}_{x}^{(r)}\left[F_{n} \prod_{k=0}^{\infty} q\left(X_{k}\right)\right]=\mathbb{E}_{x}\left[F_{n} \psi_{q}^{(r)}\left(X_{n}\right) \prod_{k=0}^{n-1} q\left(X_{k}\right)\right],
$$

as written in point ii) of Theorem 4.1.3.

Now we can prove point $i$ ), by taking for any $s \in(0,1), q(x)=\mathbf{1}_{x \neq x_{0}}+s \mathbf{1}_{x=x_{0}}$.

In this case, let us observe that $\frac{\psi_{r}\left(X_{n}\right)}{\psi_{s}\left(X_{n}\right)}$ is $\mu_{y}^{(s)}$-a.s. well-defined for all $n \geq 0$; therefore, $\mu_{y}^{(s)}\left[\frac{\psi_{r}\left(X_{n}\right)}{\psi_{s}\left(X_{n}\right)}\right]$ is well-defined and :

$$
\begin{aligned}
& \mu_{y}^{(s)}\left[\frac{\psi_{r}\left(X_{n}\right)}{\psi_{s}\left(X_{n}\right)}\right]=\mathbb{E}_{y}\left[s^{L_{n-1}^{x_{0}}} \psi_{r}\left(X_{n}\right)\right]=\mu_{y}^{(r)}\left[\left(\frac{s}{r}\right)^{L_{n-1}^{x_{0}}}\right] \\
& \underset{n \rightarrow \infty}{\rightarrow} \mu_{y}^{(r)}\left[\left(\frac{s}{r}\right)^{L_{\infty}^{x_{0}}}\right]=\mathbb{Q}_{y}^{(r)}\left[s^{L_{\infty}^{x_{0}}}\right]=\psi_{q}^{(r)}(y) .
\end{aligned}
$$

Moreover, for all $A>0$ :

$$
\mu_{y}^{(s)}\left[\frac{\psi_{r}\left(X_{n}\right)}{\psi_{s}\left(X_{n}\right)}\right]=\mu_{y}^{(s)}\left[\frac{\psi_{r}\left(X_{n}\right)}{\psi_{s}\left(X_{n}\right)} \mathbf{1}_{\psi_{s}\left(X_{n}\right) \geq A}\right]+K_{A},
$$


where :

$$
K_{A} \leq \sup \left(\frac{\psi_{r}}{\psi_{s}}\right) \cdot \mu_{y}^{(s)}\left[\psi_{s}\left(X_{n}\right) \leq A\right] \leq A \sup \left(\frac{\psi_{r}}{\psi_{s}}\right) \mathbb{E}_{y}\left[s^{\left.L_{n-1}^{x_{0}}\right]} \underset{n \rightarrow \infty}{\rightarrow} 0\right.
$$

because $\left(X_{n}\right)_{n \geq 0}$ is recurrent under $\mathbb{P}_{y}$. Hence :

$$
\begin{aligned}
& \liminf _{n \rightarrow \infty}\left(\inf _{\psi_{s}(x) \geq A} \frac{\psi_{r}(x)}{\psi_{s}(x)}\right) \mu_{y}^{(s)}\left[\psi_{s}\left(X_{n}\right) \geq A\right] \\
& \leq \liminf _{n \rightarrow \infty} \mu_{y}^{(s)}\left[\frac{\psi_{r}\left(X_{n}\right)}{\psi_{s}\left(X_{n}\right)}\right] \leq \limsup _{n \rightarrow \infty} \mu_{y}^{(s)}\left[\frac{\psi_{r}\left(X_{n}\right)}{\psi_{s}\left(X_{n}\right)}\right] \\
& \leq \limsup _{n \rightarrow \infty}\left(\sup _{\psi_{s}(x) \geq A} \frac{\psi_{r}(x)}{\psi_{s}(x)}\right) \mu_{y}^{(s)}\left[\psi_{s}\left(X_{n}\right) \geq A\right] .
\end{aligned}
$$

Now, $\inf _{\psi_{s}(x) \geq A} \frac{\psi_{r}(x)}{\psi_{s}(x)}$ and $\sup _{\psi_{s}(x) \geq A} \frac{\psi_{r}(x)}{\psi_{s}(x)}$ tend to 1 when $A$ goes to infinity and :

$$
\mu_{y}^{(s)}\left[\psi_{s}\left(X_{n}\right) \geq A\right] \rightarrow \mu_{s}^{(y)}(1)=\psi_{s}(y) .
$$

Hence, $\mu_{y}^{(s)}\left[\frac{\psi_{r}\left(X_{n}\right)}{\psi_{s}\left(X_{n}\right)}\right] \underset{n \rightarrow \infty}{\rightarrow} \psi_{s}(y)$, which implies that $\psi_{q}^{(r)}(y)=\psi_{s}(y)$.

By (4.1.21) :

$$
\begin{aligned}
\mathbb{Q}_{x}^{(r)}\left[F_{n} s^{L_{\infty}^{x_{0}}}\right] & =\mathbb{E}_{x}\left[F_{n} s^{L_{n-1}^{x_{0}}} \psi_{q}^{(r)}\left(X_{n}\right)\right]=\mathbb{E}_{x}\left[F_{n} s^{L_{n-1}^{x_{0}}} \psi_{s}\left(X_{n}\right)\right] \\
& =\mu_{x}^{(s)}\left(F_{n}\right)=\mathbb{Q}_{x}^{(s)}\left[F_{n} s^{L_{\infty}^{x_{0}}}\right] .
\end{aligned}
$$

By monotone class theorem, if $F$ is $\mathcal{F}$-measurable and positive :

$$
\mathbb{Q}_{x}^{(r)}\left(F . s^{L_{\infty}^{x_{0}}}\right)=\mathbb{Q}_{x}^{(s)}\left(F . s^{L_{\infty}^{x_{0}}}\right)
$$

for all $r, s \in(0,1)$. Now, for all $r, s, t<1$ :

$$
\mathbb{Q}_{x}^{(r)}\left(F \cdot t^{L_{\infty}^{x_{0}}}\right)=\mathbb{Q}_{x}^{(t)}\left(F \cdot t^{L_{\infty}^{x_{0}}}\right)=\mathbb{Q}_{x}^{(s)}\left(F \cdot t^{L_{\infty}^{x_{0}}}\right) .
$$

Recall that $L_{\infty}^{x_{0}}<\infty, \mathbb{Q}_{x}^{(r)}$ and $\mathbb{Q}_{x}^{(s)}$-a.s. Therefore, by monotone convergence, $\mathbb{Q}_{x}^{(r)}(F)=$ $\mathbb{Q}_{x}^{(s)}(F)$; point $i$ ) of Theorem 4.1.3 is proven, and $\mathbb{Q}_{x}$ is well-defined. By (4.1.21), point ii) is proven if $q\left(x_{0}\right)<1$. It is easy to extend this formula to the case $q\left(x_{0}\right)=1$, again by monotone convergence ; the proof of Theorem 4.1.3 is now complete.

Remark 4.1.5 The family $\left(\mathbb{Q}_{x}\right)_{x \in E}$ of $\sigma$-finite measures depends on $x_{0}$ and $\phi$, which were assumed to be fixed in this section. In the sequel of the chapter, these parameters may vary; if some confusion is possible, we will write $\left(\mathbb{Q}_{x}^{\left(\phi, x_{0}\right)}\right)_{x \in E}$ instead of $\left(\mathbb{Q}_{x}\right)_{x \in E}$.

\subsection{Some more properties of $\left(\mathbb{Q}_{x}\right)_{x \in E}$.}

4.2.1 Martingales associated with $\mathbb{Q}_{x}$.

At the beginning of this section, we extend the second point of Theorem 4.1.3 to more general functionals than functionals of the form $F_{n} \prod_{k=0}^{\infty} q\left(X_{k}\right)$. More precisely, the following result holds : 


\section{Theorem 4.2.1}

Let $F$ be a positive $\mathcal{F}$-measurable functional. For $n \geq 0, y_{0}, y_{1}, \ldots, y_{n} \in E$, let us define the quantity :

$$
\psi\left(F, y_{0}, y_{1}, \ldots, y_{n}\right)=\mathbb{Q}_{y_{n}}\left[F\left(y_{0}, y_{1}, \ldots, y_{n}=X_{0}, X_{1}, X_{2}, \ldots\right)\right] .
$$

Then, for every $\left(\mathcal{F}_{n}\right)_{n \geq 0}$-stopping time $T$, one has :

$$
\mathbb{Q}_{x}\left(F . \mathbf{1}_{T<\infty}\right)=\mathbb{E}_{x}\left[\psi\left(F, X_{0}, X_{1}, \ldots, X_{T}\right) \mathbf{1}_{T<\infty}\right] .
$$

Proof of Theorem 4.2.1: To begin with, let us suppose that $T=n$ for $n \geq 0$, and $F=r^{L_{\infty}^{x_{0}}} f_{0}\left(X_{0}\right) f_{1}\left(X_{1}\right) \ldots f_{N}\left(X_{N}\right)$ for $N>n, 0 \leq f_{i} \leq 1,0<r<1$.

One has :

$$
\begin{aligned}
\mathbb{Q}_{x}(F) & \mu_{x}^{(r)}\left[f_{0}\left(X_{0}\right) \ldots f_{N}\left(X_{N}\right)\right] \\
& =\mathbb{E}_{x}\left[f_{0}\left(X_{0}\right) \ldots f_{N}\left(X_{N}\right) r^{L_{N-1}^{x_{0}}} \psi_{r}\left(X_{N}\right)\right] \\
& =\mathbb{E}_{x}\left[f_{0}\left(X_{0}\right) \ldots f_{n-1}\left(X_{n-1}\right) r^{L_{n-1}^{x_{0}}} K\left(X_{n}\right)\right],
\end{aligned}
$$

where :

$$
\begin{aligned}
K(y) & =\mathbb{E}_{y}\left[f_{n}\left(X_{0}\right) \ldots f_{N}\left(X_{N-n}\right) r^{L_{N-n-1}^{x_{0}}} \psi_{r}\left(X_{N-n}\right)\right] \\
& =\mu_{y}^{(r)}\left[f_{n}\left(X_{0}\right) \ldots f_{N}\left(X_{N-n}\right)\right] \\
& =\mathbb{Q}_{y}\left[f_{n}\left(X_{0}\right) \ldots f_{N}\left(X_{N-n}\right) r^{L_{\infty}^{x_{0}}}\right] .
\end{aligned}
$$

Hence, for all $y_{0}, \ldots, y_{n}$ :

$$
\begin{aligned}
& f_{0}\left(y_{0}\right) \ldots f_{n-1}\left(y_{n-1}\right) r^{\sum_{k=0}^{n-1} \mathbf{1}_{y_{k}=x_{0}} K\left(y_{n}\right)} \\
& =\mathbb{Q}_{y_{n}}\left[f_{0}\left(y_{0}\right) \ldots f_{n-1}\left(y_{n-1}\right) f_{n}\left(X_{0}\right) \ldots f_{N}\left(X_{N-n}\right) r^{\sum_{k=0}^{n-1} \mathbf{1}_{y_{k}=x_{0}}+L_{\infty}^{x_{0}}}\right] \\
& =\mathbb{Q}_{y_{n}}\left[F\left(y_{0}, \ldots, y_{n}=X_{0}, X_{1}, \ldots\right)\right]=\psi\left(F, y_{0}, y_{1}, \ldots, y_{n}\right) .
\end{aligned}
$$

Therefore :

$$
\mathbb{Q}_{x}(F)=\mathbb{E}_{x}\left[\psi\left(F, X_{0}, \ldots, X_{n}\right)\right],
$$

which proves Theorem 4.2.1 for these particular functions $F$ and for $T=n$.

By monotone class theorem, we can extend (4.2.6) to the functionals $F=r^{L_{\infty}^{x_{0}}} . G$, where $G$ is any positive functional, and by monotone convergence ( $r$ increasing to 1 ), Theorem 4.2.1 is proven for all $F$.

Now, let us suppose that $T$ is a stopping time.

For $n \geq 0, \psi\left(F \mathbf{1}_{T=n}, X_{0}, X_{1}, \ldots, X_{n}\right)=\mathbf{1}_{T=n} \psi\left(F, X_{0}, \ldots, X_{n}\right)$, because $\{T=n\}$ depends only on $X_{0}, X_{1}, \ldots, X_{n}$; hence,

$$
\mathbb{Q}_{x}\left(F \mathbf{1}_{T=n}\right)=\mathbb{E}_{x}\left[\mathbf{1}_{T=n} \psi\left(F, X_{0}, \ldots, X_{n}\right)\right] .
$$

Summing from $n=0$ to infinity, we obtain the general case of Theorem 4.2.1.

Corollary 4.2.2 For any functional $F \in L^{1}\left(\mathbb{Q}_{x}\right),\left(\mathbb{E}_{x}\left[\psi\left(F, X_{0}, X_{1}, \ldots, X_{n}\right)\right]\right)_{n \geq 0}$ is a $\mathcal{F}_{n^{-}}$ martingale (with expectation $\mathbb{Q}_{x}(F)$ ).

Now, we are able to describe the properties of canonical process under $\mathbb{Q}_{x}$. 
4.2.2. Properties of the canonical process under $\mathbb{Q}_{x}$.

We have already proven that $L_{\infty}^{x_{0}}$ is almost surely finite under $\mathbb{Q}_{x}$. In fact, the following proposition gives a more general result :

Proposition 4.2.3 Under $\mathbb{Q}_{x}$, the canonical process is a.s. transient, i.e $L_{\infty}^{y_{0}}<\infty$ for all $y_{0} \in E$.

Proof of Proposition 4.2.3: Let $y_{0}$ be in $E$, and $r$ be in $(0,1)$. If, for $k \geq 1, \tau_{k}^{\left(y_{0}\right)}$ denotes the $k$-th hitting time of $y_{0}$ for the canonical process $X$, then for all $n \geq 0$ :

$$
\begin{aligned}
\mu_{x}(r)\left[L_{n-1}^{y_{0}} \geq k\right] & =\mu_{x}^{(r)}\left[\tau_{k}^{\left(y_{0}\right)}<n\right]=\mathbb{E}_{x}\left[\mathbf{1}_{\tau_{k}^{\left(y_{0}\right)}<n} r^{L_{n-1}^{x_{0}}} \psi_{r}\left(X_{n}\right)\right] \\
& =\mathbb{E}_{x}\left[\mathbf{1}_{\tau_{k}^{\left(y_{0}\right)}<n} r^{L^{x_{0}} \tau^{\left(y_{0}\right)}-1} \psi_{r}\left(y_{0}\right)\right]
\end{aligned}
$$

by strong Markov property (applied at time $\tau_{k}^{\left(y_{0}\right)} \wedge n$ ), and by the fact that $\mathbb{E}_{y_{0}}\left[r^{L_{m-1}^{x_{0}}} \psi_{r}\left(X_{m}\right)\right]=$ $\psi_{r}\left(y_{0}\right)$ for all $m \geq 0$.

Hence :

$$
\mu_{x}^{(r)}\left[L_{n-1}^{y_{0}} \geq k\right] \leq \psi_{r}\left(y_{0}\right) \mathbb{E}_{x}\left[\begin{array}{c}
L^{x_{0}} \tau_{k}^{\left(y_{0}\right)}-1 \\
\end{array}\right]
$$

by monotone convergence :

$$
\mu_{x}^{(r)}\left[L_{\infty}^{y_{0}} \geq k\right] \leq \psi_{r}\left(y_{0}\right) \mathbb{E}_{x}\left[\begin{array}{c}
L^{x_{0}} \tau_{k}^{\left(y_{0}\right)}-1 \\
\end{array}\right] \underset{k \rightarrow \infty}{\rightarrow} 0
$$

(because $L_{\tau_{k}^{\left(y_{0}\right)}}^{x_{0}} \underset{k \rightarrow \infty}{\rightarrow} \infty, \mathbb{P}_{x}$-a.s.); this implies Proposition 4.2.3.

Now, we have the following decomposition result which gives a precise description of the canonical process under $\mathbb{Q}_{y}(y \in E)$ :

Proposition 4.2.4 For all $y, y_{0} \in E$, one has :

$$
\mathbb{Q}_{y}=\mathbb{Q}_{y}^{\left[y_{0}\right]}+\sum_{k \geq 1} \mathbb{P}_{y}^{\tau_{k}^{\left(y_{0}\right)}} \circ \widetilde{\mathbb{Q}}_{y_{0}}
$$

where $\mathbb{Q}_{y}^{\left[y_{0}\right]}=\mathbf{1}_{\forall n \geq 0, X_{n} \neq y_{0}} \mathbb{Q}_{y}$ is the restriction of $\mathbb{Q}_{y}$ to trajectories which does not hit $y_{0}$, $\widetilde{\mathbb{Q}}_{y_{0}}=\mathbf{1}_{\forall n \geq 1, X_{n} \neq y_{0}} \mathbb{Q}_{y_{0}}$ is the restriction of $\mathbb{Q}_{y_{0}}$ to trajectories which does not return to $y_{0}$, and $\mathbb{P}_{y}^{\tau_{k}^{\left(y_{0}\right)}} \circ \widetilde{\mathbb{Q}}_{y_{0}}$ denotes the concatenation of $\mathbb{P}_{y}$ stopped at time $\tau_{k}^{\left(y_{0}\right)}$ and $\widetilde{\mathbb{Q}}_{y_{0}}$, i.e. the image of $\mathbb{P}_{y} \otimes \widetilde{\mathbb{Q}}_{y_{0}}$ by the functional $\Phi$ from $E^{\mathbb{N}} \times E^{\mathbb{N}}$ such that :

$$
\Phi\left(\left(z_{0}, z_{1}, \ldots, z_{n}, \ldots\right),\left(z_{0}^{\prime}, z_{1}^{\prime}, \ldots, z_{n}^{\prime}, \ldots\right)\right)=\left(z_{0}, z_{1}, \ldots, z_{\tau_{k}^{\left(y_{0}\right)}}, z_{1}^{\prime}, \ldots, z_{n}^{\prime}\right) .
$$

Proof of Proposition 4.2.4 : We apply Theorem 4.2.1 to the stopping time $T=\tau_{k}^{\left(y_{0}\right)}$, and to the functional :

$$
F=G H\left(X_{\tau_{k}^{\left(y_{0}\right)}}, X_{\tau_{k}^{\left(y_{0}\right)}+1}, \ldots\right) \mathbf{1}_{\forall u \geq 1, X_{\tau_{k}^{\left(y_{0}\right)}+u} \neq y_{0}}
$$


where $G, H$ are positive functionals such that $G \in \mathcal{F}_{\tau_{k}^{\left(y_{0}\right)}}$.

For $k \geq 1$, we obtain :

$$
\begin{aligned}
& \mathbb{Q}_{y}\left[G H\left(X_{\tau_{k}^{\left(y_{0}\right)}}, X_{\tau_{k}^{\left(y_{0}\right)}+1}, \ldots\right) \mathbf{1}_{L_{\infty}^{y_{0}}=k}\right] \\
& =\mathbb{E}_{y}\left[\mathbf{1}_{\tau_{k}^{\left(y_{0}\right)}<\infty} G\left(X_{0}, \ldots, X_{\tau_{k}^{\left(y_{0}\right)}}\right)\right] \widetilde{\mathbb{Q}}_{y_{0}}[H],
\end{aligned}
$$

which implies :

$$
\mathbb{Q}_{y}\left[G H\left(X_{\tau_{k}^{\left(y_{0}\right)}}, X_{\tau_{k}^{\left(y_{0}\right)}+1}, \ldots\right) \mathbf{1}_{L_{\infty}^{y_{0}}=k}\right]=\mathbb{E}_{y}[G] \widetilde{\mathbb{Q}}_{y_{0}}[H],
$$

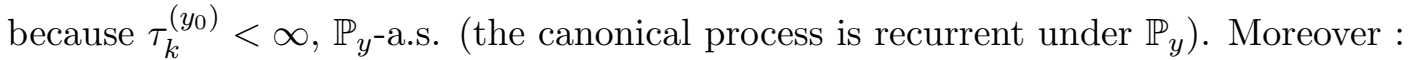

$$
\mathbb{Q}_{y}\left[H \mathbf{1}_{L_{\infty}^{y_{0}=0}}\right]=\mathbb{Q}_{y}^{\left[y_{0}\right]}(H)
$$

by definition. Now, $L_{\infty}^{y_{0}}<\infty, \mathbb{Q}_{y}$-a.s. by Proposition 4.2 .3 , so there exists $k \geq 0$ such that $L_{\infty}^{y_{0}}=k$ : the equalities (4.2.15) and (4.2.16) imply the Proposition 4.2.4 by monotone class theorem.

4.2.3 Dependence of $\mathbb{Q}_{x}$ on $x$.

The next Theorem shows that in the construction of the family $\left(\mathbb{Q}_{x}\right)_{x \in E}$, the choice of the point $x_{0}$ in $E$ is in fact not so important. More precisely, the following result holds :

Theorem 4.2.5. For all $y_{0} \in E$, let us define the function $\phi^{\left[y_{0}\right]}$ by :

$$
\phi^{\left[y_{0}\right]}(y)=\mathbb{Q}_{y}^{\left[y_{0}\right]}(1)
$$

Then the following holds :

(i) $\phi^{\left[x_{0}\right]}$ is equal to $\phi$ and for all $y_{0} \in E, \phi^{\left[y_{0}\right]}-\phi$ is a bounded function.

(ii) For all $y_{0} \in E$ :

- $\phi^{\left[y_{0}\right]}$ is finite and harmonic outside of $y_{0}$, i.e. for all $y \neq y_{0}$ :

$$
\mathbb{E}_{y}\left[\phi^{\left[y_{0}\right]}\left(X_{1}\right)\right]=\phi^{\left[y_{0}\right]}(y) .
$$

- $\phi^{\left[y_{0}\right]}\left(y_{0}\right)=0$.

- $\widetilde{\mathbb{Q}}_{y_{0}}(1)=\mathbb{E}_{y_{0}}\left[\phi^{\left[y_{0}\right]}\left(X_{1}\right)\right]$.

(iii) By point ii), $y_{0}$ and the function $\phi^{\left[y_{0}\right]}$ can be used to construct a family $\left(\mathbb{Q}_{x}^{\left(\phi^{\left[y_{0}\right]}, y_{0}\right)}\right)_{x \in E}$ of $\sigma$-finite measures by the method given in Section 4.1. Moreover, this family is equal to the family $\left(\mathbb{Q}_{x}=\mathbb{Q}_{x}^{\left(\phi, x_{0}\right)}\right)_{x \in E}$ constructed from $\phi$ and $x_{0}$.

(iv) For all $y_{0}, y \in E$, the image of the measure $\mathbb{Q}_{y}$ by the total local time at $y_{0}$ is given by the following expressions :

- $\mathbb{Q}_{y}\left[L_{\infty}^{y_{0}}=0\right]=\phi^{\left[y_{0}\right]}(y)$.

- For all $k \geq 1, \mathbb{Q}_{y}\left[L_{\infty}^{y_{0}}=k\right]=\mathbb{E}_{y_{0}}\left[\phi^{\left[y_{0}\right]}\left(X_{1}\right)\right]$. 
Proof of Theorem 4.2.5. Let $y_{0}$ and $y$ be in $E$. For all $r \in(0,1), n \geq 1$ :

$$
\begin{aligned}
& \mu_{y}^{(r)}\left[L_{n-1}^{y_{0}} \geq 1\right]=\mu_{y}^{(r)}\left[\tau_{1}^{\left(y_{0}\right)}<n\right]=\mathbb{E}_{y}\left[r^{L_{n-1}^{x_{0}}} \mathbf{1}_{\tau_{1}^{\left(y_{0}\right)}<n} . \psi_{r}\left(X_{n}\right)\right] \\
& =\mathbb{E}_{y}\left[r^{L^{L_{0}^{x_{0}}} \tau_{1}^{\left(y_{0}\right)}-1} \mathbf{1}_{\tau_{1}^{\left(y_{0}\right)}<n}\right] \psi_{r}\left(y_{0}\right)
\end{aligned}
$$

by the martingale property. Hence :

$$
\mu_{y}^{(r)}\left[L_{\infty}^{y_{0}} \geq 1\right]=\psi_{r}\left(y_{0}\right) \mathbb{E}_{y}\left[\begin{array}{c}
L^{x_{0}} \tau_{1}^{\left(y_{0}\right)}-1 \\
\end{array}\right] .
$$

If $y_{0}=x_{0}$, this implies:

$$
\mu_{y}^{(r)}\left[L_{\infty}^{x_{0}} \geq 1\right]=\psi_{r}\left(x_{0}\right)
$$

Therefore :

$$
\begin{aligned}
\phi^{\left[x_{0}\right]}(y) & =\mathbb{Q}_{y}\left[L_{\infty}^{x_{0}}=0\right]=\mu_{y}^{(r)}\left[L_{\infty}^{x_{0}}=0\right] \\
& =\mu_{y}^{(r)}(1)-\psi_{r}\left(x_{0}\right)=\psi_{r}(y)-\psi_{r}\left(x_{0}\right)=\phi(y)
\end{aligned}
$$

as written in Theorem 4.2.5. If $y_{0} \neq x_{0}$, let us define the quantities :

$$
p_{y, y_{0}}^{\left(x_{0}\right)}=\mathbb{P}_{y}\left[\tau_{1}^{y_{0}}<\tau_{1}^{x_{0}}\right],
$$

and

$$
q_{y_{0}}^{\left(x_{0}\right)}=\mathbb{P}_{x_{0}}\left[\tau_{1}^{y_{0}}>\tau_{2}^{x_{0}}\right]
$$

We have :

$$
\mathbb{P}_{y}\left[L_{\tau_{1}^{\left(y_{0}\right)}-1}^{x_{0}}=0\right]=p_{y, y_{0}}^{\left(x_{0}\right)}
$$

and, for $k \geq 1$, by strong Markov property :

$$
\mathbb{P}_{y}\left[L_{\tau_{1}^{\left(y_{0}\right)}-1}^{x_{0}}=k\right]=\left(1-p_{y, y_{0}}^{\left(x_{0}\right)}\right)\left(q_{y_{0}}^{\left(x_{0}\right)}\right)^{k-1}\left(1-q_{y_{0}}^{\left(x_{0}\right)}\right)
$$

Summing all these equalities, one obtains :

$$
\mathbb{E}_{y}\left[\begin{array}{c}
L^{L_{0}^{x_{0}}}{ }^{\tau_{1}^{\left(y_{0}\right)}-1} \\
x_{y, y_{0}}
\end{array}\right]=p_{y\left(1-p_{y, y_{0}}^{\left(x_{0}\right)}\right)\left(1-q_{y_{0}}^{\left(x_{0}\right)}\right)}
$$

and

$$
\begin{aligned}
\mu_{y}^{(r)}\left[L_{\infty}^{y_{0}} \geq 1\right] & =\left[\frac{r}{1-r} \mathbb{E}_{x_{0}}\left[\phi\left(X_{1}\right)\right]+\phi\left(y_{0}\right)\right] \ldots \\
& \ldots\left[p_{y, y_{0}}^{\left(x_{0}\right)}+\frac{r\left(1-p_{y, y_{0}}^{\left(x_{0}\right)}\right)\left(1-q_{y_{0}}^{\left(x_{0}\right)}\right)}{1-r q_{y_{0}}^{\left(x_{0}\right)}}\right] .
\end{aligned}
$$

Moreover :

$$
\mu_{y}^{(r)}(1)=\psi_{r}(y)=\frac{r}{1-r} \mathbb{E}_{x_{0}}\left[\phi\left(X_{1}\right)\right]+\phi(y)
$$


By hypothesis, there exists $n \geq 0$ such that $\mathbb{P}_{x_{0}}\left(X_{n}=y_{0}\right)>0$; it is easy to check that it implies : $q_{y_{0}}^{\left(x_{0}\right)}<1$.

Hence, by considering the difference between (4.2.28) and (4.2.29) and taking $r \rightarrow 1$, one obtains :

$$
\phi^{\left[y_{0}\right]}(y)=\mathbb{E}_{x_{0}}\left[\phi\left(X_{1}\right)\right] \frac{1-p_{y, y_{0}}^{\left(x_{0}\right)}}{1-q_{y_{0}}^{\left(x_{0}\right)}}+\left[\phi(y)-\phi\left(y_{0}\right)\right] .
$$

Therefore :

$$
\phi(y)-\phi\left(y_{0}\right) \leq \phi^{\left[y_{0}\right]}(y) \leq \frac{\mathbb{E}_{x_{0}}\left[\phi\left(X_{1}\right)\right]}{1-q_{y_{0}}^{\left(x_{0}\right)}}+\left[\phi(y)-\phi\left(y_{0}\right)\right]
$$

which implies point $i$ ) of the Theorem, and in particular the finiteness of $\phi^{\left[y_{0}\right]}$. By applying Theorem 4.2.1 to $T=1$ and $F=\mathbf{1}_{L_{\infty}^{y_{0}=0}}$, one can easily check that $\phi^{\left[y_{0}\right]}$ is harmonic everywhere except at point $y_{0}$ (where it is equal to zero).

By taking $T=1$ and $F=\mathbf{1}_{L_{\infty}^{y_{0}=1}}$, one obtains the formula: $\widetilde{\mathbb{Q}}_{y_{0}}(1)=\mathbb{E}_{y_{0}}\left[\phi^{\left[y_{0}\right]}\left(X_{1}\right)\right]$. Hence, we obtain point ii) of the theorem, and the point $i v$ ) by formula (4.2.11). Now, by taking the notation : $\mu_{y}^{(r), y_{0}}=r^{L_{\infty}^{y_{0}}} . \mathbb{Q}_{y}$, one has (for all positive and $\mathcal{F}_{n}$-measurable function $F_{n}$ ), by applying Theorem 4.3 .1 to $T=n$ and $F=F_{n} r^{L_{\infty}^{y_{0}}}$ :

$$
\mu_{y}^{(r), y_{0}}\left(F_{n}\right)=\mathbb{Q}_{y}\left[F_{n} r^{L_{\infty}^{y_{0}}}\right]=\mathbb{E}_{y}\left[F_{n} r^{L_{n-1}^{y_{0}}} \alpha\left(X_{n}\right)\right],
$$

where $\alpha(z)=\mathbb{Q}_{z}\left[r^{L_{\infty}^{y_{0}}}\right]$. By point 4 of the theorem (already proven), one has :

$$
\begin{aligned}
\alpha(z) & =\phi^{\left[y_{0}\right]}(z)+\left(\sum_{k=1}^{\infty} r^{k}\right) \mathbb{E}_{y_{0}}\left[\phi^{\left[y_{0}\right]}\left(X_{1}\right)\right] \\
& =\frac{r}{1-r} \mathbb{E}_{y_{0}}\left[\phi^{\left[y_{0}\right]}\left(X_{1}\right)\right]+\phi^{\left[y_{0}\right]}(z)
\end{aligned}
$$

Hence :

$$
\mu_{y}^{(r), y_{0}}\left(F_{n}\right)=\mathbb{E}_{y}\left[F_{n} r^{L_{n-1}^{x_{0}}}\left(\frac{r}{1-r} \mathbb{E}_{y_{0}}\left[\phi^{\left[y_{0}\right]}\left(X_{1}\right)\right]+\phi^{\left[y_{0}\right]}\left(X_{n}\right)\right)\right]
$$

This formula implies that $\mu_{y}^{(r), y_{0}}$ is the measure defined in the same way as $\mu_{y}^{(r)}$, but from the point $y_{0}$ and the function $\phi^{\left[y_{0}\right]}$, instead of the point $x_{0}$ and the function $\phi$. By putting the density $r^{-L_{\infty}^{y_{0}}}$ to this measure, one obtains the equality :

$$
\mathbb{Q}_{y}=\mathbb{Q}_{y}^{\left(\phi^{\left[y_{0}\right]}, y_{0}\right)}
$$

which completes the proof of Theorem 4.2.5.

There is also an important formula, which is a direct consequence of (4.2.1) and (4.2.5) :

Corollary 4.2.6 Let $F_{n}$ be a positive $\mathcal{F}_{n}$-measurable functional, $y, y_{0}$ be in $E$ and $g_{y_{0}}$ be the last hitting time of $y_{0}$ for the canonical process. Then the following formula holds :

$$
\mathbb{Q}_{y}\left[F_{n} \mathbf{1}_{g_{y_{0}}<n}\right]=\mathbb{E}_{y}\left[F_{n} \phi^{\left[y_{0}\right]}\left(X_{n}\right)\right]
$$

In particular, one has :

$$
\mathbb{Q}_{y}\left[F_{n} \mathbf{1}_{g_{x_{0}}<n}\right]=\mathbb{E}_{y}\left[F_{n} \phi\left(X_{n}\right)\right]
$$


and $\left(\phi^{\left[y_{a}\right]}\left(X_{1}\right), n \geq 0\right),\left(\phi\left(X_{1}\right), n \geq 0\right)$ are two $P$ submartingales.

By Theorem 4.2.5, the construction of a given family $\left(\mathbb{Q}_{x}\right)_{x \in E}$ can be obtained by taking any point $y_{0}$ instead of $x_{0}$, if the corresponding harmonic function $\phi^{\left[y_{0}\right]}$ is well-chosen.

4.2.4 Dependence of $\mathbb{Q}_{x}$ on $\phi$.

In fact, this family of $\sigma$-finite measures depends only upon the equivalent class of the function $\phi$, for an equivalence relation which will be described below. More precisely, if $\alpha$ and $\beta$ are two functions from $E$ to $\mathbb{R}_{+}$, let us write : $\alpha \simeq \beta$, iff $\alpha$ is equivalent to $\beta$ when $\alpha+\beta$ tends to infinity ; i.e, for all $\epsilon \in(0,1)$, there exists $A>0$ such that for all $x \in E, \alpha(x)+\beta(x) \geq A$ implies $1-\epsilon<\frac{\alpha(x)}{\beta(x)}<1+\epsilon$. With this definition, one has the following result :

Propostion 4.2.7 The relation $\simeq i$ an equivalence relation.

Proof of Proposition 4.2.7 The reflexivity and the symmetry are obvious, so let us prove the transitivity.

We suppose that there are three functions $\alpha, \beta, \gamma$ such that $\alpha \simeq \beta$ and $\beta \simeq \gamma$.

There exists $\epsilon: \mathbb{R}_{+} \rightarrow \mathbb{R}_{+} \cup\{\infty\}$, tending to zero at infinity, such that $\alpha+\beta \geq A$ implies $\left|\frac{\alpha}{\beta}-1\right| \leq \epsilon(A)$, and $\beta+\gamma \geq A$ implies $\left|\frac{\beta}{\gamma}-1\right| \leq \epsilon(A)$. For a given $x \in E$, let us suppose that $\alpha(x)+\gamma(x) \geq A$ for $A>4 \sup \{z, \epsilon(z) \geq 1 / 2\}$. There are two cases :

- $\alpha(x) \geq A / 2$. In this case, $\alpha(x)+\beta(x) \geq A / 2$; hence, $\left|\frac{\alpha(x)}{\beta(x)}-1\right| \leq \epsilon(A / 2) \leq 1 / 2$, which implies : $\beta(x)+\gamma(x) \geq \beta(x) \geq \alpha(x) / 2 \geq A / 4$.

Therefore : $\left|\frac{\beta(x)}{\gamma(x)}-1\right| \leq \epsilon(A / 4)$. Consequently, there exist $u$ and $v,|u| \leq \epsilon(A / 2) \leq 1 / 2$, $|v| \leq \epsilon(A / 4) \leq 1 / 2$, such that $\frac{\alpha(x)}{\gamma(x)}=(1+u)(1+v)$, which implies :

$$
\begin{aligned}
\left|\frac{\alpha(x)}{\gamma(x)}-1\right| & \leq|u|+|v|+|u v| \leq \epsilon(A / 2)+\epsilon(A / 4)+\epsilon(A / 2) \epsilon(A / 4) \\
& \leq \frac{3}{2}(\epsilon(A / 2)+\epsilon(A / 4))
\end{aligned}
$$

- $\alpha(x) \leq A / 2$. In this case, $\gamma(x) \geq A / 2$, hence we are in the same situation as in the first case if we exchange $\alpha(x)$ and $\gamma(x)$

The above inequality implies : $\alpha \simeq \gamma$, since $\epsilon(A / 2)+\epsilon(A / 4)$ tends to zero when $A$ goes to infinity. Hence, $\simeq$ is an equivalence relation.

This equivalence relation satisfies the following lemma:

Lemma 4.2.8 Let $\phi_{1}$ and $\phi_{2}$ be two functions from $E$ to $\mathbb{R}_{+}$which are equal to zero at a point $y_{0} \in E$ and which are harmonic at the other points i.e. for all $y \neq y_{0} E_{y}\left[\phi_{i}\left(X_{1}\right)\right]=$ $\phi_{i}(y), i=1,2$. If $\phi_{1} \simeq \phi_{2}$, then $\phi_{1}=\phi_{2}$.

Proof of Lemma 4.2.8 By the martingale property, for all $x \in E, A>0$ :

$$
\begin{aligned}
\phi_{1}(x) & =\mathbb{E}_{x}\left[\phi_{1}\left(X_{\left.n \wedge \tau_{1}^{\left(y_{0}\right)}\right)}\right)\right. \\
& =\mathbb{E}_{x}\left[\phi_{1}\left(X_{\left.n \wedge \tau_{1}^{\left(y_{0}\right)}\right)}\right) \mathbf{1}_{\phi_{1}\left(X_{n \wedge \tau_{1}^{\left(y_{0}\right)}}\right)+\phi_{2}\left(X_{n \wedge \tau_{1}^{\left(y_{0}\right)}}\right) \geq A}\right]+K,
\end{aligned}
$$


where $|K| \leq A \mathbb{P}_{x}\left(\tau_{1}^{\left(y_{0}\right)}>n\right)$. Now, if $\phi_{1}(y)+\phi_{2}(y) \geq A$, one has :

$$
(1-\epsilon(A)) \phi_{1}(y) \leq \phi_{2}(y) \leq(1+\epsilon(A)) \phi_{1}(y),
$$

where $\epsilon(A)$ tends to zero when $A$ tends to infinity. Therefore :

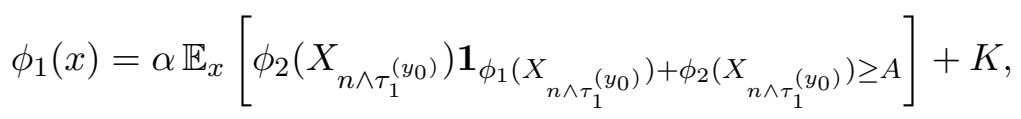

where $1-\epsilon(A) \leq \alpha \leq 1+\epsilon(A)$. Moreover :

$$
\phi_{2}(x)=\mathbb{E}_{x}\left[\phi_{2}\left(X_{\left.n \wedge \tau_{1}^{\left(y_{0}\right)}\right)} \mathbf{1}_{\phi_{1}\left(X_{n \wedge \tau_{1}^{(y)}}\right)+\phi_{2}\left(X_{n \wedge \tau_{1}^{\left(y_{0}\right)}}\right) \geq A}\right]+K^{\prime},(4\right.
$$

where $\left|K^{\prime}\right| \leq A \mathbb{P}_{x}\left(\tau_{1}^{\left(y_{0}\right)}>n\right)$. Hence :

$$
\phi_{1}(x)=\alpha\left(\phi_{2}(x)-K^{\prime}\right)+K .
$$

Now, if $A$ is fixed, $|K|+\left|K^{\prime}\right|$ tend to zero when $n$ goes to infinity. Therefore :

$$
(1-\epsilon(A)) \phi_{1}(x) \leq \phi_{2}(x) \leq(1+\epsilon(A)) \phi_{1}(x) .
$$

This inequality is true for all $A \geq 0$; hence : $\phi_{1}=\phi_{2}$, which proves Lemma 4.2.8.

We now obtain the following result, which explains why we have defined the previous equivalence relation :

Proposition 4.2.9 Let $x_{0}, y_{0}$ be in $E$, $\phi$ a positive function which is harmonic except at $x_{0}$ and equal to zero at $x_{0}, \psi$ a positive function which is harmonic except at $y_{0}$ and equal to zero at $y_{0}$. In these conditions, the family $\left(\mathbb{Q}_{x}^{\left(\phi, x_{0}\right)}\right)_{x \in E}$ of $\sigma$-finite measures is identical to the family $\left(\mathbb{Q}_{x}^{\left(\psi, y_{0}\right)}\right)_{x \in E}$ if and only if $\phi \simeq \psi$. Therefore this family can also be denoted by $\left(\mathbb{Q}_{x}^{[\phi]}\right)_{x \in E}$, where $[\phi]$ is the equivalence class of $\phi$.

Proof of Proposition 4.2.9 If the two families of measures are equal, for all $x \in E$, $\mathbb{Q}_{x}^{\left(\phi, x_{0}\right)}=\mathbb{Q}_{x}^{\left(\psi, y_{0}\right)}$. Now, it has been proven that $\psi=\mathbb{Q}_{x}^{\left(\psi, y_{0}\right)}\left(L_{\infty}^{y_{0}}=0\right)$. Hence, if $\phi^{\left[y_{0}\right]}=$ $\mathbb{Q}_{x}^{\left(\phi, x_{0}\right)}\left(L_{\infty}^{x_{0}}=0\right)$, one has $\psi=\phi^{\left[y_{0}\right]}$.

Since $\phi-\phi^{\left[y_{0}\right]}$ is bounded (point $i$ ) of Theorem 4.2.5), $\phi-\psi$ is bounded, which implies that $\phi$ is equivalent to $\psi$. On the other hand, if $\phi$ is equivalent to $\psi$, and if $\phi^{\left[y_{0}\right]}=\mathbb{Q}_{x}^{\left(\phi, x_{0}\right)}\left(L_{\infty}^{x_{0}}=0\right)$, $\psi$ and $\phi^{\left[y_{0}\right]}$ are two equivalent functions which are harmonic except at point $y_{0}$, and equal to zero at $y_{0}$. Hence, by Lemma $4.2 .8, \psi=\phi^{\left[y_{0}\right]}$, and by Theorem 4.2.5, for all $x \in E$, $\mathbb{Q}_{x}^{\left(\phi, x_{0}\right)}=\mathbb{Q}_{x}^{\left(\phi^{\left[y_{0}\right]}, y_{0}\right)}$.

Therefore, $\mathbb{Q}_{x}^{\left(\phi, x_{0}\right)}=\mathbb{Q}_{x}^{\left(\psi, y_{0}\right)}$, which proves Proposition 4.2.9.

In the next section, we give some examples of the above construction.

\subsection{Some examples.}

4.3.1 $\phi$ is a bounded function.

We remark that a function $\psi: E \rightarrow \mathbb{R}_{+}$is bounded if and only if $\psi$ is equivalent to zero. Indeed, if $\psi$ is bounded, there exists $A>0$ such that $\psi \leq A$. Hence, if $x \in E$ is such that $\psi(x)>A$, everything is true (since $x$ does not exist), in particular $\psi(x)=0$; therefore, $\psi \simeq 0$. If $\psi \simeq 0$, there exists $A>0$ such that $\psi(x)>A$ implies $\psi(x)=0$; since it is impossible to have $\psi(x)>A$ and $\psi(x)=0$ for the same $x \in E, \psi(x)>A$ is impossible : therefore, $\psi$ is bounded. 
This equivalence implies that if $\phi$ is a bounded function harmonic at all points except perhaps at a point $x_{0} \in E$, and if $\phi\left(x_{0}\right)=0$, the family of $\sigma$-finite measures $\left(\mathbb{Q}_{x}^{\left(\phi, x_{0}\right)}\right)_{x \in E}$ is dentically equal to the measure 0 . Moreover, since $\phi(x)=\mathbb{Q}_{x}^{\left(\phi, x_{0}\right)}\left[L_{\infty}^{x_{0}}=0\right]$ for all $x, \phi$ is identical to zero.

4.3.2 Example of the standard random walk.

In this case, $E=\mathbb{Z}$ and for all $x \in E, \mathbb{P}_{x}$ is the law of the standard random walk. The functions $\phi_{+}: x \rightarrow x_{+}, \phi_{-}: x \rightarrow x_{-}$and their sum $\phi: x \rightarrow|x|$ satisfies the harmonicity conditions above at point $x_{0}=0$.

Let $\left(\mathbb{Q}_{x}^{+}\right)_{x \in \mathbb{Z}},\left(\mathbb{Q}_{x}^{-}\right)_{x \in \mathbb{Z}}$ and $\left(\mathbb{Q}_{x}\right)_{x \in \mathbb{Z}}$ be the associated $\sigma$-finite measures. For all $a \in \mathbb{Z}$, let us take the notations : $\phi_{+}^{[a]}(x)=\mathbb{Q}_{x}^{+}\left[L_{\infty}^{a}=0\right], \phi_{-}^{[a]}(x)=\mathbb{Q}_{x}^{-}\left[L_{\infty}^{a}=0\right]$ and $\phi^{[a]}(x)=\mathbb{Q}_{x}\left[L_{\infty}^{a}=0\right]$. The function $\phi_{+}^{[a]}$ satisfies the harmonicity conditions at point $a$ and is equivalent to $\phi_{+}$. Now, these two properties are also satisfied by the function $x \rightarrow(x-a)_{+}$; hence, by Lemma 4.2.8, $\phi_{+}^{[a]}(x)=(x-a)_{+}$. By the same argument, $\phi_{-}^{[a]}(x)=(x-a)_{-}$and $\phi^{[a]}(x)=|x-a|$.

Therefore, we have the equalities for every positive and $\mathcal{F}_{n}$-measurable function $F_{n}$, and for every $x, a \in \mathbb{Z}$ :

$$
\begin{aligned}
\mathbb{Q}_{x}^{+}\left[F_{n} \mathbf{1}_{g_{a}<n}\right] & =\mathbb{E}_{x}\left[F_{n}\left(X_{n}-a\right)_{+}\right], \\
\mathbb{Q}_{x}^{-}\left[F_{n} \mathbf{1}_{g_{a}<n}\right] & =\mathbb{E}_{x}\left[F_{n}\left(X_{n}-a\right)_{-}\right], \\
\mathbb{Q}_{x}\left[F_{n} \mathbf{1}_{g_{a}<n}\right] & =\mathbb{E}_{x}\left[F_{n}\left|X_{n}-a\right|\right] .
\end{aligned}
$$

These equations and the fact that the canonical process is transient under $\mathbb{Q}_{x}^{+}, \mathbb{Q}_{x}^{-}, \mathbb{Q}_{x}$ characterize these measures. Moreover, by using equations (4.3.1), (4.3.2) and (4.3.3), it is not difficult to prove that for all $x \in \mathbb{Z}$, these measures are the images of $\mathbb{Q}_{0}^{+}, \mathbb{Q}_{0}^{-}$and $\mathbb{Q}_{0}$ by the translation by $x$.

Now, for all $a, x \in \mathbb{Z}$, and for all positive and $\mathcal{F}_{n}$-measurable functional $F_{n}$ :

$$
\mathbb{Q}_{x}^{+,[a]}\left[F_{n}\right]:=\mathbb{Q}_{x}^{+}\left[F_{n} \mathbf{1}_{L_{\infty}^{a}=0}\right]=\mathbb{E}_{x}\left[F_{n}\left(X_{n \wedge \tau_{1}^{(a)}}-a\right)_{+}\right] .
$$

Hence, if $x \leq a, \mathbb{Q}_{x}^{+,[a]}=0$, and if $x>a, \mathbb{Q}_{x}^{+,[a]}$ is $(x-a)$ times the law of a Bessel random walk strictly above $a$, starting at point $x$ ( $\mathrm{cf}[\mathrm{Leg}]$ for a definition of the Bessel random walk). By the same arguments, if $x \geq a, \mathbb{Q}_{x}^{-,[a]}=0$, and if $x<a, \mathbb{Q}_{x}^{-,[a]}$ is $(a-x)$ times the law of a Bessel random walk strictly below $a$, starting at point $x$. Moreover, $\mathbb{Q}_{x}^{[a]}$ is the $|x-a|$ times the law of a Bessel random walk above or below $a$, depending on the sign of $x-a$. The same kind of arguments implies that (with obvious notations) :

- $\widetilde{\mathbb{Q}}_{a}^{+}$is $1 / 2$ times the law of a Bessel random walk strictly above $a$.

- $\widetilde{\mathbb{Q}}_{a}^{-}$is $1 / 2$ times the law of a Bessel random walk strictly below $a$.

- $\widetilde{\mathbb{Q}}_{a}$ is the law of a symmetric Bessel random walk, strictly above or below $a$ with equal probability.

The equalities given by Proposition 4.2.4 are the following :

$$
\mathbb{Q}_{x}^{+}=\mathbb{Q}_{x}^{+,[a]}+\sum_{k \geq 1} \mathbb{P}_{x}^{\tau_{k}^{(a)}} \circ \widetilde{\mathbb{Q}}_{a}^{+}
$$




$$
\begin{aligned}
\mathbb{Q}_{x}^{-} & =\mathbb{Q}_{x}^{-,[a]}+\sum_{k \geq 1} \mathbb{P}_{x}^{\tau_{k}^{(a)}} \circ \widetilde{\mathbb{Q}}_{a}^{-} \\
\mathbb{Q}_{x} & =\mathbb{Q}_{x}^{[a]}+\sum_{k \geq 1} \mathbb{P}_{x}^{\tau_{k}^{(a)}} \circ \widetilde{\mathbb{Q}}_{a} .
\end{aligned}
$$

Moreover, one has :

- $\mathbb{Q}_{x}^{+}\left[L_{\infty}^{a}=0\right]=(x-a)_{+}$and $\mathbb{Q}_{x}^{+}\left[L_{\infty}^{a}=k\right]=1 / 2$ for all $k \geq 1$.

- $\mathbb{Q}_{x}^{-}\left[L_{\infty}^{a}=0\right]=(x-a)_{-}$and $\mathbb{Q}_{x}^{-}\left[L_{\infty}^{a}=k\right]=1 / 2$ for all $k \geq 1$.

- $\mathbb{Q}_{x}\left[L_{\infty}^{a}=0\right]=|x-a|$ and $\mathbb{Q}_{x}\left[L_{\infty}^{a}=k\right]=1$ for all $k \geq 1$.

Hence, by applying Theorem 4.2.1 and Corollary 4.2.2 to the functional $F=h\left(L_{\infty}^{a}\right)$ for a positive function $h$ such that $\sum_{n \in \mathbb{N}} h(n)<\infty$, and for $a \in \mathbb{Z}$, one obtains that for all $x \in \mathbb{Z}$ :

$$
\begin{aligned}
& M_{n}^{+}=\left(X_{n}-a\right)_{+} h\left(L_{n-1}^{a}\right)+\frac{1}{2} \sum_{k=L_{n-1}^{a}+1}^{\infty} h(k), \\
& M_{n}^{-}=\left(X_{n}-a\right)_{-} h\left(L_{n-1}^{a}\right)+\frac{1}{2} \sum_{k=L_{n-1}^{a}+1}^{\infty} h(k),
\end{aligned}
$$

and their sum

$$
M_{n}=\left|X_{n}-a\right| h\left(L_{n-1}^{a}\right)+\sum_{k=L_{n-1}^{a}+1}^{\infty} h(k)
$$

are martingales under the probability $\mathbb{P}_{x}$. Other martingales can be obtained by taking other functionals $F$.

4.3.3 Case of the "bang-bang" random walk.

In this case, we suppose that $E=\mathbb{N}$ and that $\left(\mathbb{P}_{x}\right)_{x \in \mathbb{N}}$ is the family of measures associated to transition probabilities : $p_{0,1}=1, p_{y, y+1}=1 / 3$ and $p_{y, y-1}=2 / 3$ for all $y \geq 1$. Informally, under $\mathbb{P}_{x}$ (for any $x \in \mathbb{N}$ ), the canonical process is a Markov process which tends to decrease when it is strictly above zero, and which increases if it is equal to zero.

The family of measures $\left(\mathbb{Q}_{x}\right)_{x \in \mathbb{N}}$ can be constructed by taking $x_{0}=0$ and $\phi(x)=2^{x}-1$ for all $x \in \mathbb{N}$.

For $y \in \mathbb{N}$, the function $\phi^{[y]}: x \rightarrow \mathbb{Q}_{x}\left[L_{\infty}^{y}=0\right]$ is harmonic except at $y$ where it is equal to zero, and it is equivalent to $\phi$.

Since the function : $x \rightarrow\left(2^{x}-2^{y}\right), \mathbf{1}_{x \geq y}$ satisfies the same properties, by Lemma 4.2 .8 : $\phi^{[y]}(x)=\left(2^{x}-2^{y}\right), \mathbf{1}_{x \geq y}$. For all $x \in \mathbb{N}$, the measure $\mathbb{Q}_{x}$ is characterized by the transience of the canonical process, and by the formula :

$$
\mathbb{Q}_{x}\left[F_{n} \mathbf{1}_{g_{a}<n}\right]=\mathbb{E}_{x}\left[F_{n}\left(2^{X_{n}}-2^{a}\right)_{+}\right],
$$

which holds for all $a, n \in \mathbb{N}$ and for every positive $\mathcal{F}_{n}$-measurable functional $F_{n}$. If one takes obvious notations, it is not difficult to prove the formula :

$$
\mathbb{Q}_{x}^{[a]}\left(F_{n}\right)=\mathbb{E}_{x}\left[F_{n}\left(2^{X}{ }^{n \wedge \tau_{1}^{(a)}}-2^{a}\right)\right] \mathbf{1}_{x \geq a},
$$


and for $n \geq 1$ :

$$
\widetilde{\mathbb{Q}}_{a}\left(F_{n}\right)=\mathbb{E}_{a}\left[F_{n}\left(2^{X \wedge \tau_{2}^{(a)}}-2^{a}\right) \mathbf{1}_{X_{1}=a+1}\right]
$$

Moreover :

- The total mass of $\mathbb{Q}_{x}^{[a]}$ is zero if $x \leq a$, and $2^{x}-2^{a}$ if $x>a$.

- The total mass of $\widetilde{\mathbb{Q}}_{a}$ is 1 if $a=0$, and $2^{a} / 3$ if $x \geq 1$.

- For $x>a$ and under the probability $\overline{\mathbb{P}}_{x}^{[a]}=\mathbb{Q}_{x}^{[a]} /\left(2^{x}-2^{a}\right)$, the canonical process is a Markov process with probability transitions : $p_{x, x+1}=\frac{2.2^{x-a}-1}{3.2^{x-a}-3}$ and $p_{x, x-1}=$ $\frac{2^{x-a}-1}{3.2^{x-a}-3}$. We remark that $p_{x, x+1}$ tends to $2 / 3$ when $x$ goes to infinity, and $p_{x, x-1}$ tends to $1 / 3$ (the opposite case as the initial transition probabilities).

- Under the probability $\frac{\widetilde{\mathbb{Q}}_{a}}{\left(2^{a} / 3\right) \mathbf{1}_{a \geq 1}+\mathbf{1}_{a=0}}$, the canonical process is a Markov process with the same transition probabilities as under $\overline{\mathbb{P}}_{x}^{[a]}$, with $X_{1}=a+1$ almost surely.

For all $a, x \in \mathbb{N}$, the image of $\mathbb{Q}_{x}$ by the total local times is given by the equalities:

$$
\mathbb{Q}_{x}\left[L_{\infty}^{a}=0\right]=\left(2^{x}-2^{a}\right) \mathbf{1}_{x>a},
$$

and for all $k \geq 1$ :

$$
\mathbb{Q}_{x}\left[L_{\infty}^{a}=k\right]=K(a),
$$

where $K(0)=1$ and $K(a)=2^{a} / 3$ for $a \geq 1$.

Moreover, if $h$ is an integrable function from $\mathbb{N}$ to $\mathbb{R}_{+}$, and if $a, x \in \mathbb{N}$,

$$
M_{n}=h\left(L_{n-1}^{a}\right)\left(2^{X_{n}}-2^{a}\right)_{+}+K(a) \sum_{k=L_{n-1}^{a}+1}^{\infty} h(k)
$$

is a martingale under the initial probability $\mathbb{P}_{x}$.

4.3.4 Case of a random walk on a tree.

We consider a random walk on a binary tree, which can be represented by the set $E=$ $\{\varnothing,(0),(1),(0,0),(0,1),(1,0),(1,1),(0,0,0), \ldots\}$ of $k$-uples of elements in $\{0,1\}(k \in \mathbb{N})$.

Obviously, $k$ is the distance to the origin $\varnothing$ of the tree.

The probability transitions of the Markov process associated to the starting family of probabilities $\left(\mathbb{P}_{x}\right)_{x \in E}$ are $p_{\varnothing,(0)}=p_{\varnothing,(1)}=1 / 2$, and for $k \geq 1: p_{\left(x_{1}, x_{2}, \ldots, x_{k}\right),\left(x_{1}, x_{2}, \ldots, x_{k-1}\right)}=1 / 2$, $p_{\left(x_{1}, \ldots, x_{k}\right),\left(x_{1}, \ldots, x_{k}, 0\right)}=p_{\left(x_{1}, \ldots, x_{k}\right),\left(x_{1}, \ldots, x_{k}, 1\right)}=1 / 4$.

In particular, under $\mathbb{P}_{x}$ (for all $x \in E$ ), the distance to the origin is a standard reflected random walk.

If the reference point $x_{0}$ is $\varnothing$, we can take for $\phi$ the distance to the origin of the tree. But there are other possible functions $\phi$ for the same point $x_{0}$. For example, if $\left(a_{0}, a_{1}, a_{2}, \ldots\right)$ is an infinite sequence of elements in $\{0,1\}$ it is possible to take for $\phi$ the function such that for all $\left(x_{0}, x_{1}, \ldots, x_{k}\right) \in E$, one has $\phi\left(x_{0}, x_{1}, \ldots, x_{k}\right)=2^{p}-1$, where $p$ is the smallest element of $\mathbb{N}$ such that $p>k$ or $x_{p} \neq a_{p}$. In particular, if $a_{p}=0$ for all $p$, one has $\phi(\varnothing)=0, \phi((0))=1$, $\phi((1))=0, \phi((0,0))=3, \phi((0,1))=1, \phi((1,0))=\phi((1,1))=0, \phi((0,0,0))=7$, etc. 
Each choice of the sequence $\left(a_{p}\right)_{p \in \mathbb{N}}$ gives a different function $\phi$ and hence a different family $\left(\mathbb{Q}_{x}^{[\phi]}\right)_{x \in E}$ of $\sigma$-finite measures.

4.3.5 Example of more general conditions for existence of $\phi$.

The following proposition gives some sufficient conditions for the existence of a function $\phi$ which satisfies the usual properties :

Proposition 4.3.1 Let $\left(\mathbb{P}_{x}\right)_{x \in E}$ be the family of probabilities associated to a discrete time Markov process on a countable set $E$. We suppose that for all $x \in E$, the set of $y \in E$ such that the transition probability $p_{x, y}$ is strictly positive is finite. Furthermore, let us consider a function $\phi$ which satisfies one of the two following conditions (for a given point $x_{0} \in E$ ) :

- There exists a function $f$ from $\mathbb{N}$ to $\mathbb{R}_{+}^{*}$ such that $f(n) / f(n+1)$ tends to 1 when $n$ goes to infinity, and such that for all $x \in E$ :

$$
\mathbb{E}_{x}\left[\tau_{1}^{\left(x_{0}\right)} \geq n\right] \underset{n \rightarrow \infty}{\sim} f(n) \phi(x) .
$$

- For all $x \in E, \mathbb{P}_{x}\left(X_{k}=x_{0}\right)$ tends to zero when $k$ tends to infinity, and:

$$
\sum_{k=0}^{N}\left[\mathbb{P}_{x_{0}}\left(X_{k}=x_{0}\right)-\mathbb{P}_{x}\left(X_{k}=x_{0}\right)\right] \underset{N \rightarrow \infty}{\rightarrow} \phi(x) .
$$

In these conditions, $\phi$ is harmonic, except at point $x_{0}$ where this function is equal to zero.

Proof of Proposition 4.3.1 Let us suppose that the first condition is satisfied. For all $x \neq x_{0}$ and for all $y \in E$ such that $p_{x, y}>0$ :

$$
\mathbb{E}_{y}\left[\tau_{1}^{\left(x_{0}\right)} \geq n\right] \underset{n \rightarrow \infty}{\sim} f(n) \phi(y) .
$$

By adding the equalities obtained for each point $y$ and multiplied by $p_{x, y}$, we obtain :

$$
\sum_{y \in E} p_{x, y} \mathbb{E}\left[\tau_{1}^{\left(x_{0}\right)} \geq n\right] \underset{n \rightarrow \infty}{\sim} f(n) \sum_{y \in E} p_{x, y} \phi(y),
$$

which implies :

$$
\mathbb{E}_{x}\left[\tau_{1}^{\left(x_{0}\right)} \geq n+1\right] \underset{n \rightarrow \infty}{\sim} f(n) \mathbb{E}_{x}\left[\phi\left(X_{1}\right)\right] .
$$

Moreover :

$$
\mathbb{E}_{x}\left[\tau_{1}^{\left(x_{0}\right)} \geq n+1\right] \underset{n \rightarrow \infty}{\sim} f(n+1) \phi(x) .
$$

By comparing these equivalences and by using the fact that $f(n)$ is equivalent to $f(n+1)$ and is strictly positive, one obtains :

$$
\phi(x)=\mathbb{E}_{x}\left[\phi\left(X_{1}\right)\right] .
$$

Since $\phi\left(x_{0}\right)$ is obviously equal to zero $\left(\mathbb{E}_{x_{0}}\left[\tau_{1}^{\left(x_{0}\right)} \geq n\right]=0\right)$, Proposition 4.3 .1 is proven if the first condition holds.

Now let us assume the second condition holds. 
If $x \neq x_{0}$, for all $y$ such that $p_{x, y}>0$ :

$$
\sum_{k=0}^{N}\left[\mathbb{P}_{x_{0}}\left(X_{k}=x_{0}\right)-\mathbb{P}_{y}\left(X_{k}=x_{0}\right)\right] \underset{N \rightarrow \infty}{\rightarrow} \phi(y) .
$$

Therefore :

$$
\sum_{y \in E} p_{x, y}\left[\sum_{k=0}^{N}\left(\mathbb{P}_{x_{0}}\left(X_{k}=x_{0}\right)-\mathbb{P}_{y}\left(X_{k}=x_{0}\right)\right)\right] \underset{N \rightarrow \infty}{\rightarrow} \sum_{y \in E} p_{x, y} \phi(y) .
$$

This equality implies :

$$
\sum_{k=0}^{N}\left[\mathbb{P}_{x_{0}}\left(X_{k}=x_{0}\right)\right]-\sum_{k=1}^{N+1}\left[\mathbb{P}_{x}\left(X_{k}=x_{0}\right)\right] \underset{N \rightarrow \infty}{\rightarrow} \mathbb{E}_{x}\left[\phi\left(X_{1}\right)\right] .
$$

Now, $\mathbb{P}_{x}\left(X_{0}=x_{0}\right)=0\left(\right.$ since $\left.x \neq x_{0}\right)$ and when $N$ goes to infinity, $\mathbb{P}_{x}\left(X_{N+1}=x_{0}\right)$ tends to zero by hypothesis. Hence :

$$
\sum_{k=0}^{N}\left[\mathbb{P}_{x_{0}}\left(X_{k}=x_{0}\right)-\mathbb{P}_{x}\left(X_{k}=x_{0}\right)\right] \underset{N \rightarrow \infty}{\rightarrow} \mathbb{E}_{x}\left[\phi\left(X_{1}\right)\right],
$$

which implies :

$$
\phi(x)=\mathbb{E}_{x}\left[\phi\left(X_{1}\right)\right],
$$

as written in Proposition 4.3.1.

Remark 4.3.2 If the condition:

$$
\sum_{k=0}^{N}\left[\mathbb{P}_{x_{0}}\left(X_{k}=x_{0}\right)-\mathbb{P}_{x}\left(X_{k}=x_{0}\right)\right] \underset{N \rightarrow \infty}{\rightarrow} \phi(x)
$$

is satisfied for a function $\phi$, then $\phi$ is automatically positive. Indeed :

$$
\begin{aligned}
\sum_{k=0}^{N}\left[\mathbb{P}_{x_{0}}\left(X_{k}=x_{0}\right)-\mathbb{P}_{x}\left(X_{k}=x_{0}\right)\right] & =\mathbb{E}_{x_{0}}\left[\sharp\left\{k \leq N, X_{k}=x_{0}\right\}\right] \\
& -\mathbb{E}_{x}\left[\sharp\left\{k \leq N, X_{k}=x_{0}\right\}\right],
\end{aligned}
$$

where, by strong Markov property :

$$
\begin{aligned}
\mathbb{E}_{x_{0}}\left[\sharp\left\{k \leq N, X_{k}=x_{0}\right\}\right] & \geq \mathbb{E}_{x}\left[\sharp\left\{k \leq \tau_{1}^{\left(x_{0}\right)}+N, X_{k}=x_{0}\right\}\right] \\
& \geq \mathbb{E}_{x}\left[\sharp\left\{k \leq N, X_{k}=x_{0}\right\}\right] .
\end{aligned}
$$

4.3.6 Case of the standard random walk in $\mathbb{Z}^{2}$.

In this case, $E=\mathbb{Z}^{2}$ and $\left(\mathbb{P}_{x}\right)_{x \in \mathbb{Z}^{2}}$ is the family of probabilities associated to the standard random walk. If we take $x_{0}=(0,0)$, the problem is to find a function $\phi$ which satisfies the usual properties of harmonicity : it can be solved by using Proposition 4.3.1.

More precisely, by doing some classical computations, we can prove that for all $(x, y) \in \mathbb{Z}^{2}$, and for all $k \in \mathbb{N}$ :

$$
\mathbb{P}_{(x, y)}\left[X_{k}=(0,0)\right]=\mathbf{1}_{k \equiv x+y(\bmod .2)} \frac{C}{k+1}+\epsilon_{(x, y)}(k),
$$


where for all $(x, y), k^{2} \epsilon_{(x, y)}(k)$ is bounded and $C$ is a universal constant. Therefore, for all $N$ :

$$
\begin{aligned}
\sum_{k=0}^{N} \mathbb{P}_{(x, y)}\left[X_{k}=(0,0)\right] & =C \sum_{k \leq N, k \equiv x+y(\text { mod. } 2)} \frac{1}{k+1}+\sum_{k=0}^{N} \epsilon_{(x, y)}(k) \\
& =\frac{C}{2} \log (N)+\eta_{(x, y)}(N),
\end{aligned}
$$

where for all $(x, y) \in \mathbb{Z}^{2}, \eta_{(x, y)}(N)$ converges to a limit $\eta_{(x, y)}(\infty)$ when $N$ goes to infinity. Therefore :

$$
\sum_{k=0}^{N}\left[\mathbb{P}_{(0,0)}\left(X_{k}=(0,0)\right)-\mathbb{P}_{(x, y)}\left(X_{k}=(0,0)\right)\right] \underset{N \rightarrow \infty}{\rightarrow} \phi((x, y)):=\eta_{(0,0)}(\infty)-\eta_{(x, y)}(\infty)
$$

By Proposition 4.3.1, the function $\phi$ is harmonic except at $(0,0)$, and can be used to construct the family of probabilities $\left(\mathbb{Q}_{(x, y)}\right)_{(x, y) \in \mathbb{Z}^{2}}$, as in dimension one. Moreover, it is not difficult to check that $\mathbb{Q}_{(x, y)}$ is the image of $\mathbb{Q}_{(0,0)}$ by the translation of $(x, y)$. 


\section{Index of main notations}

\section{Chap. 1}

$\Omega=\mathcal{C}\left(\mathbb{R}_{+} \rightarrow \mathbb{R}\right)$ : the space of continuous functions from $\mathbb{R}_{+}$to $\mathbb{R}$

$\left(X_{t}, t \geq 0\right)$ : the set of coordinates in this space

$\left(\mathcal{F}_{t}, t \geq 0\right)$ : the natural filtration

$\mathcal{F}_{\infty}=\underset{t \geq 0}{\vee} \mathcal{F}_{t}$

$b\left(\mathcal{F}_{t}\right)$ : the space of bounded real valued $\mathcal{F}_{t}$ measurable functions

$\left(W_{x}, x \in \mathbb{R}\right):$ the set of Wiener measures on $\left(\Omega, \mathcal{F}_{\infty}\right)$

$W=W_{0}$

$W_{x}(Y)$ : the expectation of the r.v. $Y$ with respect to $W_{x}$

$\left(L_{t}^{y}, y \in \mathbb{R}, t \geq 0\right)$ : the bicontinuous process of local times

$\left(L_{t}:=L_{t}^{0}, t \geq 0\right)$ the local time at level 0

$\left(\tau_{l}:=\inf \left\{t \geq 0 ; L_{t}>l\right\}, l \geq 0\right):$ the right continuous inverse of $\left(L_{t}, t \geq 0\right)$

$q$ a positive Radon measure on $\mathbb{R}$

$\mathcal{I}$ : the set of positive Radon measure on $\mathbb{R}$ s.t. $\int_{-\infty}^{\infty}(1+|x|) q(d x)<\infty$

$\delta_{a}:$ the Dirac measure at $a$

$\left(A_{t}^{(q)}:=\int_{0}^{t} q\left(X_{s}\right) d s=\int_{\mathbb{R}} L_{t}^{y} q(d y), t \geq 0\right):$ the additive functional associated with $q$

$\left(W_{x, \infty}^{(q)}, x \in \mathbb{R}\right)$ : the family of probabilities on $\left(\Omega, \mathcal{F}_{\infty}\right)$ obtained by Feynman-Kac penalisation

$\left(M_{x, s}^{(q)}, s \geq 0\right)$ : the martingale density of $W_{x, \infty}^{(q)}$ with respect to $W_{x}$

$\varphi_{q}:$ a scale function

$\varphi_{q}, \varphi_{q}^{ \pm}$: solutions of the Sturm-Liouville equation $\varphi^{\prime \prime}=q \varphi$

$\left(\mathbf{W}_{x}, x \in \mathbb{R}\right):$ a family of positive $\sigma$-finite measures on $\left(\Omega, \mathcal{F}_{\infty}\right)$

$L^{1}\left(\Omega, \mathcal{F}_{\infty}, \mathbf{W}\right)\left(\right.$ resp. $\left.L_{+}^{1}\left(\Omega, \mathcal{F}_{\infty}, \mathbf{W}\right)\right)$ : the Banach space of r.v.

$\mathbf{W}$-integrable (resp. positive and $\mathbf{W}$-integrable)

$\left(M_{t}(F), t \geq 0\right):$ a martingale associated with $F \in L^{1}\left(\Omega, \mathcal{F}_{\infty}, \mathbf{W}\right)$

$g_{a}:=\sup \left\{s \geq 0 ; X_{s}=a\right\} \quad ; \quad g_{0}=g$

$g_{a}^{(t)}:=\sup \left\{s \leq t, X_{s}=a\right\} \quad ; \quad g_{0}^{(t)}=g^{(t)}$

$\sigma_{a}:=\sup \left\{s \geq 0 ; X_{s} \in[-a, a]\right\} ; \sigma_{a, b}:=\sup \left\{s \geq 0 ; X_{s} \in[a, b]\right\}$

$f_{Z}^{(P)}$ : density of the r.v. $Z$ under $P$

$T$ a $\left(\mathcal{F}_{t}, t \geq 0\right)$ stopping time

$P_{0}^{(3)}$ (resp. $\widetilde{P}_{0}^{(3)}$ ) : the law of a 3 -dimensional Bessel process (resp. of the opposite of a 3 -dimensional Bessel process) started at 0

$P_{0}^{(3, \mathrm{sym})}=\frac{1}{2}\left(P_{0}^{(3)}+\widetilde{P}_{0}^{(3)}\right)$

$W_{0}^{\tau_{l}}$ : the law of a 1-dimensional Brownian motion stopped at $\tau_{l}$

$\Pi_{0,0}^{(t)}$ : the law of the Brownian bridge $\left(b_{u}, 0 \leq u \leq t\right)$ of length $t$ (and s.t. $b_{0}=b_{t}=0$ )

$\omega \circ \widetilde{\omega}$ : the concatenation of $\omega$ and $\widetilde{\omega}(\omega, \widetilde{\omega} \in \Omega)$

$\omega=\left(\omega_{t}, \omega^{t}\right)$ : decomposition of $\omega$ before and after $t$

$\Gamma^{+}=\left\{\omega \in \Omega ; X_{t} \underset{t \rightarrow \infty}{\longrightarrow} \infty\right\}, \Gamma^{-}=\left\{\omega \in \Omega ; X_{t}(\omega) \underset{t \rightarrow \infty}{\longrightarrow}-\infty\right\}$

$\mathbf{W}^{+}=1_{\Gamma^{+}} \cdot \mathbf{W}, \quad \mathbf{W}^{-}=1_{\Gamma^{-}} \cdot \mathbf{W}$

$W^{F}\left(F \in L_{+}^{1}\left(\Omega, \mathcal{F}_{\infty}, \mathbf{W}\right)\right)$ : the finite measure defined on $\left(\Omega, \mathcal{F}_{\infty}\right)$ by : $W^{F}(G)=\mathbf{W}(F \cdot G)$

$\left(\nu_{x}^{(q)}, x \in \mathbb{R}\right)$ : a family of $\sigma$-finite measures associated with the additive functional $\left(A_{+}^{(q)}, t \geq 0\right)$ 
$\left(Z_{t}, t \geq 0\right):$ a positive Brownian supermartingale

$Z_{\infty}:=\lim _{t \rightarrow \infty} Z_{t} \quad W$ a.s. $; z_{\infty}:=\lim _{t \rightarrow \infty} \frac{Z_{t}}{1+\left|X_{t}\right|} \quad \mathbf{W}$ a.s.

$\left(\Delta_{t}(F), t \geq 0\right),\left(\Sigma_{t}(F), t \geq 0\right):$ two quasimartingales associated with $F \in L^{1}\left(\Omega, \mathcal{F}_{\infty}, \mathbf{W}\right)$

$(\Phi, s \geq 0):$ a predictable positive process

$\left(k_{s}(F), s \geq 0\right)$ a predictable process such that $W\left(F \mid \mathcal{F}_{g}\right)=k_{g}(F)\left(F \in L_{+}^{1}\left(\Omega, \mathcal{F}_{\infty}, \mathbf{W}\right)\right.$

$\left(\chi_{t}, t \geq 0\right):$ a $\mathcal{C}\left(\mathbb{R}_{+} \rightarrow \mathbb{R}\right)$ Markov process valued

$\left(\mathbb{P}_{t}, t \geq 0\right):$ the semigroup associated to $\left(\chi_{t}, t \geq 0\right)$

$\mathbf{W}_{x}^{a, b}=a \mathbf{W}_{x}^{+}=b \mathbf{W}_{x}^{-}$

$\widetilde{\mathbf{W}}^{a, b}=\int d x \mathbf{W}_{x}^{a, b}:$ is an invariant measure for $\left(\chi_{t}, t \geq 0\right)$

$\widetilde{\Omega}=\mathcal{C}\left(\mathbb{R} \rightarrow \mathbb{R}_{+}\right):$the space of continuous functions from $\mathbb{R}$ to $\mathbb{R}_{+}$

$<q, l>:=\int_{\mathbb{R}} l(x) q(d x), q \in \mathcal{I}, l \in \widetilde{\Omega}$

$\mathcal{L}: \Omega \rightarrow \widetilde{\Omega}$ defined by $\mathcal{L}\left(X_{t}, t \geq 0\right)=\left(L_{\infty}^{y}, y \in \mathbb{R}\right)$

$\left(Q_{t}, t \geq 0\right)$ : the semigroup associated with the Markov process $\left(\left(X_{t}, L_{t}^{\bullet}\right), t \geq 0\right)$ which is

$\mathbb{R} \times \widetilde{\Omega}$ valued

$\mathcal{G}$ : the infinitesimal generator of $\left(Q_{t}, t \geq 0\right)$

$\left(\widetilde{\Lambda}^{a, b}, a, b \geq 0\right):$ a family of invariant measures for $\left(\left(X_{t}, L_{t}^{\bullet}\right), t \geq 0\right)$

$\left(\Lambda_{x}, x \in \mathbb{R}\right):$ a family of positive and $\sigma$-finite on $\widetilde{\Omega}$

$\theta: \mathbb{R} \times \widetilde{\Omega} \rightarrow \widetilde{\Omega}$ defined by $\theta(x, l)(y)=l(x-y) \quad(x, y \in \mathbb{R}, l \in \widetilde{\Omega})$

$\left(L_{t}^{X_{t} \bullet}, t \geq 0\right)$ : a Markov process $\widetilde{\Omega}$ valued

$\left(\bar{Q}_{t}, t \geq 0\right)$ : the semigroup associated with $\left(L_{t}^{X_{t^{-}}}, t \geq 0\right)$

$\overline{\mathcal{G}}$ : the infinitesimal generator of $\left(\bar{Q}_{t}, t \geq 0\right)$

$\mathbf{\Lambda}^{a, b}=a \boldsymbol{\Lambda}^{+}+b \boldsymbol{\Lambda}^{-}$

\section{Chap. 2}

$\Omega=\mathcal{C}\left(\mathbb{R}_{+} \rightarrow \mathbb{C}\right):$ the space of continuous functions from $\mathbb{R}_{+}$to $\mathbb{C}$

$\left(X_{t}, t \geq 0\right)$ : the coordinate process on $\Omega$

$\left(W_{x}^{(2)}, x \in \mathbb{C}\right)$ the set of Wiener measures ; $W_{0}^{(2)}=W^{(2)}$

$\mathcal{J}$ : the set of positive Radon measures on $\mathbb{C}$ with compact support

$\left(A_{t}^{(q)}:=\int_{0}^{t} q\left(X_{s}\right) d s, t \geq 0\right):$ the additive functional associated with $\left.q \in \mathcal{J}\right)$

$\left(W_{z, \infty}^{(2, q)}, z \in \mathbb{C}\right)$ : the set of probabilities obtained by Feynman-Kac penalisations associated with $q \in \mathcal{I} ; W_{0, \infty}^{(2, q)}=W_{\infty}^{(2, q)}$

$\left(M_{s}^{(2, q)}, s \geq 0\right)$ : the martingale density of $W_{z, \infty}^{(2, q)}$ with respect to $W_{z}^{(2)}$

$\varphi_{q}:$ a solution of Sturm-Liouville equation $\Delta \varphi=q \varphi$

$\Delta$ : the Laplace operator

$\left(\mathbf{W}_{z}^{(2)}, z \in \mathbb{C}\right)$ : a family of positive and $\sigma$-finite measures on $\left(\Omega, \mathcal{F}_{\infty}\right)$

$\mathbf{W}_{0}^{(2)}=\mathbf{W}^{(2)}$

$C$ : the unit circle in $\mathbb{C}$

$\left(L_{t}^{(C)}, t \geq 0\right)$ : the continuous local time process on $C$

$\left(\tau_{l}^{(C)}, l \geq 0\right)$ : the right continuous inverse of $\left(L_{t}^{(C)}, t \geq 0\right)$

$\left(R_{t}, t \geq 0\right)$ : the process solution of (2.2.6)

$P_{1}^{(2, \log )}:$ the law of process $\left(R_{t}, t \geq 0\right)$ 
$\left(\rho_{u}, u \geq 0\right)$ : a 3-dimensional Bessel process starting from 0.

$\left(H_{t}:=\int_{0}^{t} \frac{d s}{R_{s}^{2}}, t \geq 0\right)$

$g_{C}:=\sup \left\{s \geq 0 ; X_{t} \in C\right\}$

$W_{0}^{\left(2, \tau_{l}^{(C)}\right)}$ : the law of a $\mathbb{C}$-valued Brownian motion stopped at $\tau_{l}^{(C)}$

$\widetilde{P}_{1}^{(2, \log )}$ : the law of $\left(X_{g_{C}+s}, s \geq 0\right)$

$\nabla:$ the gradient operator

$K_{0}$ : the Bessel Mc Donald function with index 0

$T_{1}^{(3)}:=\inf \left\{u ; \rho_{u}=1\right\}$

$\left(R_{t}^{(\delta)}, t \geq 0\right)$ : the process solution of (2.3.19)

$\left(M_{t}^{(2)}(F), t \geq 0\right):$ the Brownian martingale associated with $F \in L^{1}\left(\Omega, \mathcal{F}_{\infty}, \mathbf{W}^{(2)}\right)$

\section{Chap. 3}

$\Omega=\mathcal{C}\left(\mathbb{R}_{+} \rightarrow \mathbb{R}_{+}\right):$the space of continuous functions from $\mathbb{R}_{+}$to $\mathbb{R}_{+}$

$S:$ the scale function

$m$ : the speed measure

$\left(X_{t}, t \geq 0, \mathbb{P}_{x}, x \in \mathbb{R}_{+}\right)$: the canonical process associated with $S$ and $m$

$\left(\mathcal{F}_{t}, t \geq 0\right):$ the natural filtration $; \mathcal{F}_{\infty}=\underset{t \geq 0}{\vee} \mathcal{F}_{t}$

$L=\frac{d}{d m} \frac{d}{d S}$ : the infinitesimal generator of $\left(X_{t}, t \geq 0\right)$

$p(t, x, \bullet)$ : the density of $X_{t}$ under $\mathbb{P}_{x}$ with respect to $m$

$\left(L_{t}^{y}, t \geq 0, y \geq 0\right)$ : the jointly continuous family of local times of $X$

$\left(L_{t}, t \geq 0\right)$ : the local time process a level 0

$\left(\tau_{l}, l \geq 0\right)$ : the right continuous inverse of $\left(L_{t}, t \geq 0\right)$

$p_{x}^{\tau_{l}}$ : the law of process $\left(X_{t}, t \geq 0\right)$ started at $x$ and stopped at $\tau_{l}$

$g_{y}:=\sup \left\{t \geq 0 ; X_{t}=y\right\} \quad ; \quad g:=g_{0}$

$g_{y}^{(t)}:=\sup \left\{s \leq t ; X_{s}=y\right\} \quad ; \quad g^{(t)}:=g_{0}^{(t)}$

$T_{0}:=\inf \left\{t \geq 0 ; X_{t}=0\right\}$

$\left(\widehat{X}_{t}, t \geq 0\right):$ the process $\left(X_{t}, t \geq 0\right)$ killed at $T_{0}$

$\widehat{p}(t, x, \bullet)$ : the density of $\widehat{X}_{t}$ under $P_{x}$ with respect to $m$

$\left(P_{x}^{\uparrow}, x \in \mathbb{R}_{+}\right)$: the laws of $X$ conditionned not to vanish $; P^{\uparrow}:=P_{0}^{\uparrow}$

$f_{y, 0}(t)$ defined by : $f_{y, 0}(t) d t=P_{y}\left(T_{0} \in d t\right)=P_{0}^{\uparrow}\left(g_{y} \in d t\right)$

$\mathbf{W}^{*}$ a $\sigma$-finite measure on $\left(\Omega, \mathcal{F}_{\infty}\right)$

$\Pi_{0}^{(t)}$ : the law of the bridge of lenght $t$

$\mathbf{W}_{g}^{*}$ : the restriction of $\mathbf{W}^{*}$ to $\mathcal{F}_{g}$

$\left(M_{t}^{(\lambda, x)}=\frac{1+\frac{\lambda}{2} S\left(X_{t}\right)}{1+\frac{\lambda}{2} S(x)} \cdot e^{-\frac{\lambda}{2} L_{t}}, t \geq 0\right):$ the density martingale of $P_{x, \infty}^{(\lambda)}$ with respect to $P_{x}$ $\left(M_{t}^{*}(F), t \geq 0\right)$ : the positive $\left(\left(\mathcal{F}_{t} \geq 0\right), P_{0}\right)$ martingale associated with $F \in L^{1}\left(\Omega, \mathcal{F}_{\infty}, \mathbf{W}^{*}\right)$ $\left(P_{x}^{(-\alpha)}, x \geq 0\right)$ : the family of laws of a Bessel process with dimension $d=2(1-\alpha)$

$(0<d<2,0<\alpha<1)$ started at $x$

$\mathbf{W}^{(-\alpha)}$ : the measure $\mathbf{W}^{*}$ in the particular case of Bessel processes with index $(-\alpha)$

$(0<\alpha<1)$

$\Pi_{0}^{(-\alpha, t)}$ : the law of the Bessel bridge with index $(-\alpha)$ and length $t$

$P_{x}^{\left(-\alpha, \tau_{l}\right)}$ : the law of a Bessel process with index $(-\alpha)$ started at $x$ and stopped at $\tau_{l}$ 
$\varphi_{q}:$ a solution of the Sturm-Liouville equation :

$$
\frac{1}{2} \varphi^{\prime \prime}(r)+\frac{1-2 \alpha}{2 r} \varphi^{\prime}(r)=\frac{1}{2} \varphi(r) q(r) \quad r \geq 0
$$

with $q$ a positive Radon measure with compact support $\left(m_{u}, 0 \leq u \leq 1\right)$ : the Bessel meander with dimension $d$ $P_{0}^{\left(\frac{\delta}{2}-1, m, \swarrow\right)}$ : the law of the process obtained by putting back to back two Bessel processes with index $\left(\frac{\delta}{2}-1\right)$, starting from 0 and stopped when they reach level $m$

\section{Chap. 4}

$E$ : a countable set $\left(X_{n}, n \geq 0\right)$ : the canonical process on $E^{\mathbb{N}}$ $\left(\mathcal{F}_{n}, n \geq 0\right):$ the natural filtration, $\mathcal{F}_{\infty}=\underset{n \geq 0}{\vee} \mathcal{F}_{n}$ $\left(P_{x}, x \in E\right):$ the family of probabilities associated to Markov process $\left(X_{n}, n \geq 0\right)$ s.t. $P\left(X_{n+1}=z \mid X_{n}=y\right)=p_{y, z}$ and $P_{x}\left(X_{0}=x\right)=1$

$\phi$ : a positive function from $E$ to $\mathbb{R}_{+}$, harmonic with respect to $P$, except at the point $x_{0}$ and such that $\phi\left(x_{0}\right)=0$

$\psi_{r}(x):=\frac{r}{1-r} E_{x_{0}}\left(\phi\left(X_{1}\right)\right)+\phi(x) \quad(r \in] 0,1[, x \in E)$ $\left(\mu_{x}^{(r)}, x \in E, r \in\right] 0,1[):$ a family of probabilities on $\left(E^{\mathbb{N}}, \mathcal{F}_{\infty}\right)$

$q$ : a function from $E$ to $[0,1]$ such that $\{q<1\}$ is a finite set $\left(E_{x}\left[\psi\left(F, X_{0}, X_{1}, \cdots, X_{n}\right)\right], n \geq 0\right):$ the $\left(\left(\mathcal{F}_{n}, n \geq 0\right), P_{x}\right)$ martingale associated with $F \in L^{1}\left(\Omega, \mathcal{F}_{\infty}, \mathbb{Q}_{x}\right)$

$\left(L_{k}^{y}=\sum_{m=0}^{k} 1_{X_{m}=y}, k \geq 0\right):$ the local time of $\left(X_{n}, n \geq 0\right)$ at level $y\left(\right.$ with $\left.L_{-1}^{y}=0\right)$ $\tau_{k}^{(y)}:$ the $k$ hitting time of $y$

$\left(\tau_{k}^{(y)}, k \geq 0\right)$ : the inverse of $\left(L_{k}^{y}, k \geq 0\right)$

$\mathbb{Q}_{y}^{\left[y_{0}\right]}$ : the restriction of $\mathbb{Q}_{y}$ to trajectories which does not hit $y_{0}$

$\widetilde{Q}_{y}$ : the restriction of $\mathbb{Q}_{y}$ to trajectories which does not return to $y$

$P_{x}^{\tau_{k}^{\left(y_{0}\right)}}$ : the law of the Markov chain $\left(X_{n}, n \geq 0\right)$ starting from $x$ and stopped at $\tau_{k}^{\left(y_{0}\right)}$

$2 \widetilde{Q}_{a}^{+}$: the law of a Bessel random walk strictly above $a$

$2 \widetilde{Q}_{a}^{-}$: the law of a Bessel random walk strictly below $a$

$\widetilde{Q}_{a}:=\widetilde{Q}_{a}^{+}+\widetilde{Q}_{a}^{-}$

$g_{a}:=\sup \left\{n \geq 0 ; X_{n}=a\right\}$

$\phi^{\left[y_{0}\right]}$ defined by $\phi^{\left[y_{0}\right]}(y)=\mathbb{Q}_{y}^{\left[y_{0}\right]}(1)$

$\simeq$ : the equivalent relation defined in subsection 4.2.4

$\mathbb{Q}_{x}=\left(\frac{1}{r}\right) L_{\infty}^{x_{0}} \mu_{x}^{(r)}$ independent of $\left.r \in\right] 0,1[$

$\mathbb{Q}_{x}^{\left(\psi, y_{0}\right)}$ : the finite $\sigma$-finite measure $\mathbb{Q}_{x}$ constructed from the point $y_{0}$ and the function $\psi$ $\mathbb{Q}_{x}^{[\psi]}$ : the measure $Q_{x}^{\left(\psi, y_{0}\right)}$ where $[\psi]$ denotes the equivalent class of $\psi$ 


\section{References}

[AY1] J. Azma, M. Yor Une solution simple au problme de Skorokhod. In Sminaire de Probabilits (Univ. Strasbourg, Strasbourg 1977/78). LNM 721, Springer, Berlin, p. 90-115, 1979

[AY2] J. Azma, M. Yor Sur les zros des martingales continues. In Sminaire de Probabilits XXVI. LNM 1526, Springer, Berlin, Heidelberg, New-York, p. 248-306, 1992

[Bi] P. Biane Decomposition of Brownian trajectories and some applications. In A. Badrikian, P.A. Meyer, J.A. Yan (Eds). Probability and Statistics. Rencontres franco-chinoises en Probabilits et Statistiques, Proceedings of Wuhan meeting, World Scientific, Singapore, p. 51-76, 1993

[Be] J. Bertoin Lvy processes. Cambridge Tracts in Math. Cambridge, Vol. 121, Univ. Press, Cambridge 1996

[BY] P. Biane, M. Yor Valeurs principales associes aux temps locaux browniens. Bul. Sci. Math. 111 p. 23-101 (1987)

[BS] A.N. Borodin, P. Salminen Handbook of Brownian motion - facts and formulae - . Prob. and its Applications. Birkhuser, Basel, 2d edition 2002

[C] L. Chaumont Excursion normalise, mandre et pont pour les processus de Lvy stables. Bull. Sci. Math. 121(5) p. 377-403 (1997)

[D-MY] C. Donati-Martin, M. Yor Some measure valued Markov processes attached to occupation time of Brownian motion. Bernoulli 6(1) p. 63-72 (2000)

[D-MRVY] C. Donati-Martin, B. Roynette, P. Vallois, M. Yor On constants related to the choice of the local time at 0 , and the corresponding It measure for Bessel processes with dimension $d=2(1-\alpha), 0<\alpha<1$. To appear in Studia Sci. Math. Hungarica, (2008)

[Fe] W. Feller An introduction to probability theory and its applications. Vol. II. J. Wiley and Sons Inc., New-York 1966

[F] H. Fllmer The exit measure of a supermartingale. Z. Wahrscheinlichkeitstheorie und Verw. Gebiete 21 p. 154-166 (1972)

[J] T. Jeulin Semi-martingales et grossissement d'une filtration. LNM 721, Springer, Berlin, Heidelberg, New-York, 1980

[JY] T. Jeulin, M. Yor Grossissement de filtrations : exemples et applications. LNM 1118, Springer, Berlin, Heidelberg, New-York. Papers from the seminar on stochastic calculus held at University Paris VI, Paris 1982/83

[Kn] F.B. Knight Brownian local time and taboo processes. Trans. Amer. Math. Soc. 143 p. 173-185 (1969)

[Ko] S. Kotani Asymptotics for expectations of multiplicative functionals of one-dimensional Brownian motion. preprint (nov. 2006) 
[KS] U. Kchler, P. Salminen On spectral measures of strings and excursions of quasidiffusions. In J. Azma, P.A. Meyer and M. Yor, editors. Sminaire de Probabilits XXIII. LNM 1372, Springer, Berlin, p. 490-502, 1989

[L] N.N. Lebedev Special functions and their applications. Revised English Ed., Dover Publications Inc, New-York 1965

[LG1] J.F. Le Gall Une approche lmentaire des thormes de dcomposition de Williams. In Sminaire de Probabilits XX. LNM 1204, Springer, Berlin, p. 447-464, 1986

[LG2] J.F. Le Gall Sur le temps local d'intersection du mouvement brownien plan et la mthode de renormalisation de Varadhan. In Sminaire de Probabilits XIX. LNM 1123, Springer, Berlin, Heidelberg, New-York, p. 314-331, 1985

[M] P.A. Meyer Probabilits et potentiel. Publication de l'Institut de Math. de l'Univ. de Strasbourg n XIV, Actualits Scientifiques et Industrielles 1318, Hermann, Paris 1966

[N1] J. Najnudel Temps locaux et pnalisations browniennes. Thse de l'Universit Paris 6, juin 2007

[N2] J. Najnudel Pnalisations de l'araigne brownienne. Ann. Inst. Fourier 57(4), p. 1063-1093 (2007)

[N3] J. Najnudel On the construction of Edward's measures on $\mathcal{C}\left(\mathbb{R}_{+}, \mathbb{R}\right)$. Submitted (2008)

[O] J. Oblój A complete characterization of local martingales which are functions of brownian motion and its supremum. Bernoulli 12(6) p. 955-969 (2006)

[Pi] J. Pitman One dimensional Brownian motion and the three dimensional Bessel process. Adv. in Appl. Prob. 7(3), p. 511-526 (1975)

[PY1] J. Pitman, M. Yor Asymptotic laws of planar Brownian motion. Ann. Prob. 14, p. 733-779 (1986)

[PY2] J. Pitman, M. Yor Decomposition at the maximum for excursions and bridges of one dimensional diffusions. In N. Ikeda, S. Watanabe, M. Fukushima, H. Kunita (Eds). It's stochastic calculus and probability theory. Springer, Berlin, Heidelberg, New-York, p. 293-310, 1996

[Pr] C. Profeta, Thesis in preparation (2008)

[R] K.M. Rao Quasi-martingales. Math. Scand. 24, p. 79-92 (1969)

[ReY] D. Revuz, M. Yor Continuous martingales and Brownian motion. Grundlehren der Math. Wissenschaften 293, Springer, Berlin, Third Edition 1999

[RVY, I] B. Roynette, P. Vallois, M. Yor Limiting laws associated with Brownian motion perturbed by normalized exponential weights, I. Studia Sci. Math. Hungarica 43(2), p. 171-246 (2006)

[RVY, II] B. Roynette, P. Vallois, M. Yor Limiting laws associated with Brownian motion perturbed by its maximum, minimum and local time II. Studia Sci. Math. Hungarica 43(3), p. 295-360 (2006) 
[RVY, III] B. Roynette, P. Vallois, M. Yor Limiting laws for long Brownian bridges perturbed by their one-sided maximum III. Period. Math. Hungar. 50(1-2), p. 247-280 (2005)

[RVY IV] B. Roynette, P. Vallois, M. Yor Some extensions of Pitman and Ray-Knight theorems for penalized Brownian motion and their local time IV. Studia Sci. Math. Hungarica 44(4), p. 469-517 (2007)

[RVY, V] B. Roynette, P. Vallois, M. Yor Penalizing a BES(d) process $(0<\alpha<2)$ with a function of its local time $V$. To appear in Studia Sci. Math. Hungarica (2008)

[RVY, VI] B. Roynette, P. Vallois, M. Yor Penalisation of multidimensional Brownian motion. Submitted to ESAIM (2007)

[RVY, VII] B. Roynette, P. Vallois, M. Yor Brownian penalisations related to excursions lengths. Submitted to Annales de l'IHP (2007)

[RY, VIII] B. Roynette, M. Yor Ten penalisations of Brownian motion involving its one-sided supremum until first and last passage times VIII. In preparation (2008)

[RY, IX] B. Roynette, M. Yor Local limit theorems for Brownian additive functionals and penalizations of Brownian paths IX. In preparation (2008)

[RVY, X] B. Roynette, P. Vallois, M. Yor Penalisations of Brownian motions with its maximum and minimum processes as weak forms of Skorokhod embeddings. Submitted (2008)

[RVY, J] B. Roynette, P. Vallois, M. Yor Some penalisations of the Wiener measure. Japan Jour. of Math. 1(1), p. 263-290 (2006)

[RY, M] B. Roynette, M. Yor Penalising Brownian paths ; Rigorous results and Metatheorems. Monograph - Submitted (2007)

[S] F. Spitzer Some theorems concerning 2-dimensional Brownian motion.. Trans. Amer. Math. Soc. 87, p. 187-197 (1958)

[Sa1] P. Salminen One dimensional diffusions and their exit spaces. Math. Scand. 54, p. 209-220 (1984)

[Sa2] P. Salminen On last exit decomposition of linear diffusions. Studia Sci. Math. Hungarica 33, p. 251-262 (1997)

[SV] P. Salminen, P. Vallois On subexponentiality of the Lvy measure of the diffusion inverse local time, with applications to penalisations. . In preparation (2008)

[SVY] P. Salminen, P. Vallois, M. Yor On the excursion theory for linear diffusions. Japan Jour. of Math. 2, p. 91-127 (2007)

[SY] P. Salminen, M. Yor Tanaka formula for symmetric Lvy processes. In Sminaire de Probabilits XL. LNM 1899, Springer, Berlin, p. 265-285, 2007

[W1] J. Westwater On Edwards model for long polymer chains. Comm. Math. Phys. 72, p. 131-174 (1980) 
[W2] J. Westwater On Edwards model for polymer chains. II. The self-consistent potential. Comm. Math. Phys. 79, p. 53-73 (1981)

[W3] J. Westwater On Edwards model for polymer chains. III. Borel summability. Comm. Math. Phys. 84(4), p. 459-470 (1982)

[YYY] K. Yano, Y. Yano, M. Yor Penalising symmetric stable Lvy paths. In preparation (2008) 